\title{
Cochrane
}

Library

Cochrane Database of Systematic Reviews

\section{Topical and device-based treatments for fungal infections of the toenails (Review)}

Foley K, Gupta AK, Versteeg S, Mays R, Villanueva E, John D

Foley K, Gupta AK, Versteeg S, Mays R, Villanueva E, John D.

Topical and device-based treatments for fungal infections of the toenails.

Cochrane Database of Systematic Reviews 2020, Issue 1. Art. No.: CD012093.

DOI: 10.1002/14651858.CD012093.pub2.

www.cochranelibrary.com

Topical and device-based treatments for fungal infections of the toenails (Review) 
TABLE OF CONTENTS

HEADER

ABSTRACT

PLAIN LANGUAGE SUMMARY

SUMMARY OF FINDINGS

BACKGROUND

Figure 1.

OBJECTIVES

METHODS

RESULTS

Figure 2.

Figure 3.

Figure 4.

DISCUSSION

AUTHORS' CONCLUSIONS

ACKNOWLEDGEMENTS

REFERENCES

CHARACTERISTICS OF STUDIES

DATA AND ANALYSES

Analysis 1.1. Comparison 1 Ciclopirox $8 \%$ lacquer vs vehicle, Outcome 1 Complete cure: 36 - 52 weeks.

Analysis 1.2. Comparison 1 Ciclopirox $8 \%$ lacquer vs vehicle, Outcome 2 Adverse events (directly related to treatment). .........

Analysis 1.3. Comparison 1 Ciclopirox 8\% lacquer vs vehicle, Outcome 3 Mycological cure: 36 - 52 weeks. ..............................

Analysis 2.1. Comparison 2 Efinaconazole $10 \%$ solution vs vehicle, Outcome 1 Complete cure: 40 - 52 weeks.

Analysis 2.2. Comparison 2 Efinaconazole $10 \%$ solution vs vehicle, Outcome 2 Adverse events.

Analysis 2.3. Comparison 2 Efinaconazole $10 \%$ solution vs vehicle, Outcome 3 Mycological cure: 40 - 52 weeks.

Analysis 2.4. Comparison 2 Efinaconazole 10\% solution vs vehicle, Outcome 4 Clinical cure: 40 - 52 weeks.

Analysis 3.1. Comparison 3 Tavaborole $5 \%$ solution vs vehicle, Outcome 1 Complete cure: 36 - 52 weeks.

Analysis 3.2. Comparison 3 Tavaborole $5 \%$ solution vs vehicle, Outcome 2 Adverse events.

Analysis 3.3. Comparison 3 Tavaborole $5 \%$ solution vs vehicle, Outcome 3 Adverse events (directly related to treatment). .......

Analysis 3.4. Comparison 3 Tavaborole $5 \%$ solution vs vehicle, Outcome 4 Mycological cure: 36 - 52 weeks.

Analysis 4.1. Comparison 4 P-3051 (ciclopirox 8\% hydrolacquer) vs comparators, Outcome 1 Complete cure: 48 - 52 weeks. ....

Analysis 4.2. Comparison 4 P-3051 (ciclopirox $8 \%$ hydrolacquer) vs comparators, Outcome 2 Adverse events.

Analysis 4.3. Comparison 4 P-3051 (ciclopirox 8\% hydrolacquer) vs comparators, Outcome 3 Mycological cure: 48 - 52 weeks. ..

Analysis 4.4. Comparison 4 P-3051 (ciclopirox 8\% hydrolacquer) vs comparators, Outcome 4 Mycological cure (participants with dermatophyte infections): 48 - 52 weeks.

Analysis 5.1. Comparison 5 1064-nm Nd:YAG laser vs no treatment/sham, Outcome 1 Adverse events.

Analysis 5.2. Comparison 5 1064-nm Nd:YAG laser vs no treatment/sham, Outcome 2 Mycological cure: 12 weeks.

Analysis 5.3. Comparison 5 1064-nm Nd:YAG laser vs no treatment/sham, Outcome 3 Mycological cure: 36 - 52 weeks. ...........

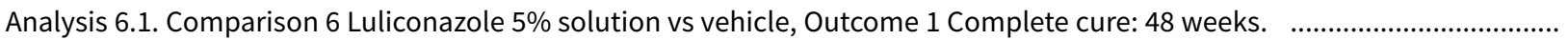

Analysis 6.2. Comparison 6 Luliconazole $5 \%$ solution vs vehicle, Outcome 2 Adverse events.

Analysis 6.3. Comparison 6 Luliconazole $5 \%$ solution vs vehicle, Outcome 3 Mycological cure: 48 weeks.

Analysis 7.1. Comparison 7 Spirulina extract vs placebo, Outcome 1 Mycological cure: 12 - 36 weeks.

Analysis 8.1. Comparison 8 Resin lacquer $30 \%$ vs amorolfine 5\% lacquer, Outcome 1 Mycological cure: 12 - 36 weeks.

Analysis 8.2. Comparison 8 Resin lacquer $30 \%$ vs amorolfine $5 \%$ lacquer, Outcome 2 Mycological cure: 36 - 52 weeks. ............

Analysis 8.3. Comparison 8 Resin lacquer $30 \%$ vs amorolfine $5 \%$ lacquer, Outcome 3 Compliance (self-report).

Analysis 9.1. Comparison 9 Amorolfine $5 \%$ nail lacquer: nail varnish vs no nail varnish, Outcome 1 Adverse events.

Analysis 9.2. Comparison 9 Amorolfine $5 \%$ nail lacquer: nail varnish vs no nail varnish, Outcome 2 Mycological cure: 12 weeks. .

Analysis 10.1. Comparison 10 Acetic acid-based peelable nail polish vs amorolfine $5 \%$ lacquer, Outcome 1 Mycological cure: 12 - 36 weeks.

Analysis 11.1. Comparison 11 Photodynamic therapy vs intense pulsed light therapy, Outcome 1 Complete cure: 12 - 36 weeks. 
Analysis 12.1. Comparison 12 Fractional laser and photodynamic therapy vs fractional laser and amorolfine $5 \%$ lacquer, Outcome 1 Mycological cure: 12 weeks.

Analysis 12.2. Comparison 12 Fractional laser and photodynamic therapy vs fractional laser and amorolfine $5 \%$ lacquer, Outcome 2 Mycological cure: 36 - 52 weeks.

ADDITIONAL TABLES 
[Intervention Review]

\section{Topical and device-based treatments for fungal infections of the toenails}

Kelly Foley ${ }^{1}$, Aditya K Gupta ${ }^{1}$, Sarah Versteeg${ }^{1}$, Rachel Mays ${ }^{1}$, Elmer Villanueva², Denny John ${ }^{3}$

1Mediprobe Research Inc., London, Canada. 2Department of Public Health, Xi'an Jiaotong-Liverpool University, Suzhou, China.

${ }^{3}$ Campbell Collaboration, New Delhi, India

Contact address: Kelly Foley, Mediprobe Research Inc., 645 Windermere Road, London, ON, N5X 2P1, Canada. kellyafoley@gmail.com.

Editorial group: Cochrane Skin Group.

Publication status and date: New, published in Issue 1, 2020.

Citation: Foley K, Gupta AK, Versteeg S, Mays R, Villanueva E, John D. Topical and device-based treatments for fungal infections of the toenails. Cochrane Database of Systematic Reviews 2020, Issue 1. Art. No.: CD012093. DOI: 10.1002/14651858.CD012093.pub2.

Copyright $(2020$ The Cochrane Collaboration. Published by John Wiley \& Sons, Ltd.

\section{A B S T R A C T}

\section{Background}

Onychomycosis refers to fungal infections of the nail apparatus that may cause pain, discomfort, and disfigurement. This is an update of a Cochrane Review published in 2007; a substantial amount of new research warrants a review exclusively on toenails.

\section{Objectives}

To assess the clinical and mycological effects of topical drugs and device-based therapies for toenail onychomycosis.

\section{Search methods}

We searched the following databases up to May 2019: the Cochrane Skin Group Specialised Register, CENTRAL, MEDLINE, Embase and LILACS. We also searched five trials registers, and checked the reference lists of included and excluded studies for further references to relevant randomised controlled trials.

\section{Selection criteria}

Randomised controlled trials of topical and device-based therapies for onychomycosis in participants with toenail onychomycosis, confirmed by positive cultures, direct microscopy, or histological nail examination. Eligible comparators were placebo, vehicle, no treatment, or an active topical or device-based treatment.

\section{Data collection and analysis}

We used standard methodological procedures expected by Cochrane. Primary outcomes were complete cure rate (normal-looking nail plus fungus elimination, determined with laboratory methods) and number of participants reporting treatment-related adverse events.

\section{Main results}

We included 56 studies (12,501 participants, average age: 27 to 68 years), with mainly mild-to-moderate onychomycosis without matrix involvement (where reported). Participants had more than one toenail affected. Most studies lasted 48 to 52 weeks; $23 \%$ reported disease duration (variable). Thirty-five studies specifically examined dermatophyte-caused onychomycosis. Forty-three studies were carried out in outpatient settings. Most studies assessed topical treatments, $9 \%$ devices, and $11 \%$ both.

We rated three studies at low risk of bias across all domains. The most common high-risk domain was performance bias. We present results for key comparisons, where treatment duration was 36 or 48 weeks, and clinical outcomes were measured at 40 to 52 weeks.

Based on two studies (460 participants), compared with vehicle, ciclopirox $8 \%$ lacquer may be more effective in achieving complete cure (risk ratio (RR) 9.29, 95\% confidence interval (Cl) 1.72 to 50.14; low-quality evidence) and is probably more effective in achieving mycological cure (RR 3.15, 95\% Cl 1.93 to 5.12; moderate-quality evidence). Ciclopirox lacquer may lead to increased adverse events, 
commonly application reactions, rashes, and nail alteration (e.g. colour, shape). However, the $95 \% \mathrm{Cl}$ indicates that ciclopirox lacquer may actually make little or no difference (RR $1.61,95 \% \mathrm{Cl} 0.89$ to 2.92 ; low-quality evidence).

Efinaconazole $10 \%$ solution is more effective than vehicle in achieving complete cure (RR $3.54,95 \% \mathrm{Cl} 2.24$ to $5.60 ; 3$ studies, 1716 participants) and clinical cure (RR 3.07, 95\% Cl 2.08 to 4.53; 2 studies, 1655 participants) (both high-quality evidence) and is probably more effective in achieving mycological cure (RR 2.31, 95\% Cl 1.08 to 4.94; 3 studies, 1716 participants; moderate-quality evidence). Risk of adverse events (such as dermatitis and vesicles) was slightly higher with efinaconazole (RR 1.10, 95\% $\mathrm{Cl} 1.01$ to $1.20 ; 3$ studies, 1701 participants; high-quality evidence). No other key comparison measured clinical cure.

Based on two studies, compared with vehicle, tavaborole $5 \%$ solution is probably more effective in achieving complete cure (RR 7.40 , $95 \% \mathrm{Cl} 2.71$ to $20.24 ; 1198$ participants), but probably has a higher risk of adverse events (application site reactions were most commonly reported) (RR 3.82, 95\% Cl 1.65 to 8.85; 1186 participants (both moderate-quality evidence)). Tavaborole improves mycological cure (RR $3.40,95 \% \mathrm{Cl} 2.34$ to $4.93 ; 1198$ participants; high-quality evidence).

Moderate-quality evidence from two studies (490 participants) indicates that P-3051 (ciclopirox $8 \%$ hydrolacquer) is probably more effective than the comparators ciclopirox $8 \%$ lacquer or amorolfine $5 \%$ in achieving complete cure (RR $2.43,95 \% \mathrm{Cl} 1.32$ to 4.48 ), but there is probably little or no difference between the treatments in achieving mycological cure (RR $1.08,95 \% \mathrm{Cl} 0.85$ to 1.37 ). We found no difference in the risk of adverse events (RR $0.60,95 \% \mathrm{Cl} 0.19$ to 1.92; 2 studies, 487 participants; low-quality evidence). The most common events were erythema, rash, and burning.

Three studies (112 participants) compared 1064-nm Nd:YAG laser to no treatment or sham treatment. We are uncertain if there is a difference in adverse events (very low-quality evidence) (two studies; 85 participants). There may be little or no difference in mycological cure at 52 weeks (RR 1.04, 95\% Cl 0.59 to 1.85; 2 studies, 85 participants; low-quality evidence). Complete cure was not measured.

One study (293 participants) compared luliconazole 5\% solution to vehicle. We are uncertain whether luliconazole leads to higher rates of complete cure (very low-quality evidence). Low-quality evidence indicates there may be little or no difference in adverse events (RR $1.02,95 \% \mathrm{Cl} 0.90$ to 1.16 ) and there may be increased mycological cure with luliconazole; however, the $95 \% \mathrm{Cl}$ indicates that luliconazole may make little or no difference to mycological cure (RR $1.39,95 \% \mathrm{Cl} 0.98$ to 1.97). Commonly-reported adverse events were dry skin, paronychia, eczema, and hyperkeratosis, which improved or resolved post-treatment.

\section{Authors' conclusions}

Assessing complete cure, high-quality evidence supports the effectiveness of efinaconazole, moderate-quality evidence supports P-3051 (ciclopirox $8 \%$ hydrolacquer) and tavaborole, and low-quality evidence supports ciclopirox $8 \%$ lacquer. We are uncertain whether luliconazole $5 \%$ solution leads to complete cure (very low-quality evidence); this outcome was not measured by the 1064-nm Nd:YAG laser comparison. Although evidence supports topical treatments, complete cure rates with topical treatments are relatively low.

We are uncertain if 1064-nm Nd:YAG laser increases adverse events compared with no treatment or sham treatment (very low-quality evidence). Low-quality evidence indicates that there is no difference in adverse events between P-3051 (ciclopirox hydrolacquer), luliconazole $5 \%$ solution, and their comparators. Ciclopirox $8 \%$ lacquer may increase adverse events (low-quality evidence). High- to moderate-quality evidence suggests increased adverse events with efinaconazole $10 \%$ solution or tavaborole $5 \%$ solution.

We downgraded evidence for heterogeneity, lack of blinding, and small sample sizes. There is uncertainty about the effectiveness of devicebased treatments, which were under-represented; $80 \%$ of studies assessed topical treatments, but we were unable to evaluate all of the currently relevant topical treatments.

Future studies of topical and device-based therapies should be blinded, with patient-centred outcomes and an adequate sample size. They should specify the causative organism and directly compare treatments.

\section{PLAIN LANGUAGE SUMMARY}

\section{Are topical and device-based treatments effective in people with fungal infections of the toenails?}

\section{Review question}

We reviewed evidence about the effect of topical and device-based treatments for fungal infections of the toenails (toenail onychomycosis) when compared against each other, placebo (an identical but inactive treatment), vehicle (inactive ingredients that help deliver an active treatment), or no treatment. We assessed adults, whose infection was diagnosed based on studying nail samples.

\section{Background}

Toenail onychomycosis causes pain, discomfort, and disfigurement. Topical and device-based treatments can have less likelihood of drug interactions or side effects than oral drugs. Antifungal medications are either fungistatic (inhibiting fungal growth) or fungicidal (killing fungal pathogens). The shared goal of devices (e.g. laser, photodynamic therapy) is fungus destruction. 


\section{Study characteristics}

In searches up to May 2019, we found 56 studies including 12,501 men or women (average age: 27 to 68 years) who had mainly mild-tomoderate toenail onychomycosis. Onychomycosis duration was under-reported, but varied from months to years. Approximately $63 \%$ of the studies assessed onychomycosis caused by dermatophytes (fungi). Most studies lasted 48 to 52 weeks and were conducted in an outpatient setting. The studies used either device-based or topical treatments, including lacquers and creams, alone or in combination, compared to each other, to no treatment, to vehicle, or to placebo.

\section{Key results}

For the following key results, treatment lasted 36 or 48 weeks, and outcomes were measured at 40 to 52 weeks (side effects were measured throughout the study).

Compared to vehicle (no treatment), efinaconazole 10\% topical solution is better at achieving complete cure (i.e. normal-looking nail coupled with fungus elimination determined using laboratory methods) (high-quality evidence). Tavaborole $5 \%$ solution (when compared to vehicle) and P-3051 (ciclopirox 8\% hydrolacquer) (when compared to two other treatments: ciclopirox $8 \%$ lacquer or amorolfine 5\%) are probably better at achieving this outcome (both moderate-quality evidence). Ciclopirox $8 \%$ lacquer may lead to higher complete cure rates than vehicle, but rates are low (not all patients can be expected to achieve complete cure) (low-quality evidence).

Ciclopirox $8 \%$ lacquer and efinaconazole $10 \%$ are probably more effective at eliminating the fungus (mycological cure) than vehicle, but for P-3051 (ciclopirox $8 \%$ hydrolacquer) there is probably little or no difference compared to the two comparator treatments (all moderatequality evidence). Tavaborole $5 \%$ improves mycological cure compared to vehicle (high-quality evidence).

We found no evidence of a difference in side effects, including redness, rash, and burning, between P-3051 (ciclopirox $8 \%$ hydrolacquer) and the two other treatments (low-quality evidence), and ciclopirox $8 \%$ lacquer may increase side effects, including application-site reactions, rashes, and changes in the nail compared with vehicle, although treatment effects vary, so it is possible that it may actually make little or no difference (low-quality evidence). Compared to vehicle, participants were slightly more likely to experience side effects (commonly dermatitis and fluid-filled sacs) with efinaconazole $10 \%$ (high-quality evidence) and probably more likely to experience side effects with tavaborole 5\% (commonly, application-site reactions, such as dermatitis, redness, and pain) (moderate-quality evidence).

We are uncertain of the effect of luliconazole $5 \%$ on complete cure when compared to vehicle (very low-quality evidence); there may be little or no difference between these groups in side effects (dry skin, eczema, and thickening of the skin were commonly reported, but improved after stopping treatment), and luliconazole 5\% solution might increase mycological cure; however, the effects of this treatment vary, so it is possible that it may actually make little or no difference to mycological cure (both low-quality evidence).

Three studies compared laser to no treatment or sham treatment, and there may be little or no difference in mycological cure (low-quality evidence). Complete cure was not measured, and we are uncertain if there is a difference in side effects between groups (very low-quality evidence).

Efinaconazole $10 \%$ solution is more effective in achieving clinical cure than vehicle (high-quality evidence); none of the other key comparisons measured this outcome.

\section{Quality of the evidence}

We base our conclusions on varied evidence quality. For complete cure, mycological cure, and side effects, quality ranged from low to high, with very low-quality evidence found for three key results.

Many studies were small, had design issues, and did not directly compare therapies. No studies reported quality of life. 
SUMMARY OF FINDINGS

Summary of findings for the main comparison. Ciclopirox $8 \%$ lacquer compared to vehicle for fungal infections of the toenails

Ciclopirox $8 \%$ lacquer compared to Vehicle for fungal infections of the toenails

Patient or population: people with fungal infections of the toenails

Setting: outpatient clinics

Intervention: ciclopirox $8 \%$ lacquer

Comparison: vehicle

\begin{tabular}{|c|c|c|c|c|c|c|}
\hline \multirow[t]{2}{*}{ Outcomes } & \multicolumn{2}{|c|}{ Anticipated absolute effects ${ }^{\star}(95 \% \mathrm{CI})$} & \multirow{2}{*}{$\begin{array}{l}\text { Relative ef- } \\
\text { fect } \\
(95 \% \mathrm{CI})\end{array}$} & \multirow{2}{*}{$\begin{array}{l}\text { № of partici- } \\
\text { pants } \\
\text { (studies) }\end{array}$} & \multirow{2}{*}{$\begin{array}{l}\text { Quality of the } \\
\text { evidence } \\
\text { (GRADE) }\end{array}$} & \multirow[t]{2}{*}{ Comments } \\
\hline & $\begin{array}{l}\text { Risk with Ve } \\
\text { hicle }\end{array}$ & $\begin{array}{l}\text { Risk with Ciclopirox } \\
8 \% \text { lacquer }\end{array}$ & & & & \\
\hline \multirow[t]{2}{*}{ Complete cure: 48 weeks } & \multicolumn{2}{|c|}{ Study population } & \multirow{2}{*}{$\begin{array}{l}\text { RR } 9.29 \\
(1.72 \text { to } \\
50.14)\end{array}$} & \multirow{2}{*}{$\begin{array}{l}460 \\
\text { (2 RCTs) }\end{array}$} & \multirow{2}{*}{$\begin{array}{l}\oplus \oplus \oplus \ominus \\
\text { Low }^{a}\end{array}$} & \multirow[t]{2}{*}{ NNTB $=3$} \\
\hline & 4 per 1000 & $\begin{array}{l}41 \text { per } 1000 \\
(8 \text { to } 219)\end{array}$ & & & & \\
\hline \multirow{2}{*}{$\begin{array}{l}\text { Adverse events (directly related to } \\
\text { treatment, collected over the course } \\
\text { of the studies; they are not mea- } \\
\text { sured/reported for specific time } \\
\text { points) }\end{array}$} & \multicolumn{2}{|c|}{ Study population } & \multirow{2}{*}{$\begin{array}{l}\text { RR } 1.61 \\
\text { (0.89 to } 2.92 \text { ) }\end{array}$} & \multirow{2}{*}{$\begin{array}{l}460 \\
\text { (2 RCTs) }\end{array}$} & \multirow{2}{*}{$\begin{array}{l}\oplus \oplus \oplus \ominus \\
\text { Low }^{b}\end{array}$} & \multirow{2}{*}{$\begin{array}{l}\text { The most commonly reported } \\
\text { adverse events were application } \\
\text { site reactions (transient tingling, } \\
\text { burning, or pain with treatment } \\
\text { use), rashes (mild erythema in the } \\
\text { skin surrounding the nail), and al- } \\
\text { terations in nail colour or shape. } \\
\text { These adverse reactions did not re- } \\
\text { quire additional treatment. }\end{array}$} \\
\hline & 70 per 1000 & $\begin{array}{l}112 \text { per } 1000 \\
(62 \text { to } 204)\end{array}$ & & & & \\
\hline \multirow[t]{2}{*}{ Mycological cure: 48 weeks } & \multicolumn{2}{|c|}{ Study population } & \multirow{2}{*}{$\begin{array}{l}\text { RR } 3.15 \\
\text { (1.93 to } 5.12 \text { ) }\end{array}$} & \multirow{2}{*}{$\begin{array}{l}460 \\
\text { (2 RCTs) }\end{array}$} & \multirow{2}{*}{$\begin{array}{l}\oplus \oplus \oplus \ominus \\
\text { Moderateb }\end{array}$} & \multirow[t]{2}{*}{ NNTB $=2$} \\
\hline & 96 per 1000 & $\begin{array}{l}303 \text { per } 1000 \\
\text { (185 to } 492)\end{array}$ & & & & \\
\hline Clinical cure - not measured & - & - & - & - & - & - \\
\hline
\end{tabular}

*The risk in the intervention group (and its $95 \%$ confidence interval) is based on the assumed risk in the comparison group and the relative effect of the intervention (and its $95 \% \mathrm{Cl})$.

CI: Confidence interval; RR: Risk ratio; NNTB: Number needed to treat for an additional beneficial outcome 
High quality: We are very confident that the true effect lies close to that of the estimate of the effect

Moderate quality: We are moderately confident in the effect estimate: The true effect is likely to be close to the estimate of the effect, but there is a possibility that it is sub-

stantially different

Low quality: Our confidence in the effect estimate is limited: The true effect may be substantially different from the estimate of the effect

Very low quality: We have very little confidence in the effect estimate: The true effect is likely to be substantially different from the estimate of effect

aDowngraded by two levels to low-quality evidence: one level due to imprecision as there are very large and variable confidence intervals across both studies, with low event rates (fewer than 100). Also downgraded by one level due to risk of bias, as most information is from studies at unclear risk of bias.

bDowngraded by two levels to low-quality evidence: one level due to imprecision since the $95 \% \mathrm{Cl}$ includes both a meaningful increase in risk, and no increase in risk, and one level due to risk of bias, as most information is from studies at unclear risk of bias.

\section{Summary of findings 2 . Efinaconazole $10 \%$ solution compared to vehicle for fungal infections of the toenails}

\section{Efinaconazole $10 \%$ solution compared to vehicle for fungal infections of the toenails}

Patient or population: People with fungal infections of the toenails

Setting: outpatient clinics

Intervention: Efinaconazole 10\% solution

Comparison: Vehicle

\begin{tabular}{|c|c|c|c|c|c|c|}
\hline \multirow[t]{2}{*}{ Outcomes } & \multicolumn{2}{|c|}{ Anticipated absolute effects ${ }^{\star}(95 \% \mathrm{Cl})$} & \multirow{2}{*}{$\begin{array}{l}\text { Relative effect } \\
(95 \% \mathrm{CI})\end{array}$} & \multirow{2}{*}{$\begin{array}{l}\text { № of partici- } \\
\text { pants } \\
\text { (studies) }\end{array}$} & \multirow{2}{*}{$\begin{array}{l}\text { Quality of the } \\
\text { evidence } \\
\text { (GRADE) }\end{array}$} & \multirow[t]{2}{*}{ Comments } \\
\hline & $\begin{array}{l}\text { Risk with Vehi- } \\
\text { cle }\end{array}$ & $\begin{array}{l}\text { Risk with Efinaconazole } \\
10 \% \text { solution }\end{array}$ & & & & \\
\hline \multirow{2}{*}{$\begin{array}{l}\text { Complete cure: } 40 \text { to } 52 \\
\text { weeks }\end{array}$} & \multicolumn{2}{|l|}{ Study population } & \multirow{2}{*}{$\begin{array}{l}\text { RR } 3.54 \\
\text { (2.24 to } 5.60)\end{array}$} & \multirow{2}{*}{$\begin{array}{l}1716 \\
\text { (3 RCTs) }\end{array}$} & \multirow{2}{*}{$\begin{array}{l}\oplus \oplus \oplus \oplus \\
\text { High }\end{array}$} & \multirow[t]{2}{*}{ NNTB $=2$} \\
\hline & 46 per 1000 & $\begin{array}{l}162 \text { per } 1000 \\
(102 \text { to } 256)\end{array}$ & & & & \\
\hline \multirow{2}{*}{$\begin{array}{l}\text { Adverse events (collect- } \\
\text { ed over the course of the } \\
\text { studies; they are not mea- } \\
\text { sured/reported for specif- } \\
\text { ic time points) }\end{array}$} & \multicolumn{2}{|l|}{ Study population } & \multirow{2}{*}{$\begin{array}{l}\text { RR } 1.10 \\
\text { (1.01 to } 1.20)\end{array}$} & \multirow{2}{*}{$\begin{array}{l}1701 \\
\text { (3 RCTs) }\end{array}$} & \multirow{2}{*}{$\begin{array}{l}\oplus \oplus \oplus \oplus \\
\mathrm{High}\end{array}$} & \multirow[t]{2}{*}{-} \\
\hline & 600 per 1000 & $\begin{array}{l}660 \text { per } 1000 \\
(606 \text { to } 720)\end{array}$ & & & & \\
\hline \multirow{2}{*}{$\begin{array}{l}\text { Mycological cure: } 40 \text { to } 52 \\
\text { weeks }\end{array}$} & \multicolumn{2}{|l|}{ Study population } & \multirow{2}{*}{$\begin{array}{l}\text { RR } 2.31 \\
\text { (1.08 to } 4.94)\end{array}$} & \multirow{2}{*}{$\begin{array}{l}1716 \\
\text { (3 RCTs) }\end{array}$} & \multirow{2}{*}{$\begin{array}{l}\oplus \oplus \oplus \ominus \\
\text { Moderate } a\end{array}$} & NNTB $=2$ \\
\hline & 196 per 1000 & $\begin{array}{l}454 \text { per } 1000 \\
\text { ( } 212 \text { to } 970)\end{array}$ & & & & $\begin{array}{l}\text { We performed a sensitivity } \\
\text { analysis omitting Tschen } 2013 \\
\text { from the analysis, with this pro- } \\
\text { viding high-quality evidence }\end{array}$ \\
\hline
\end{tabular}




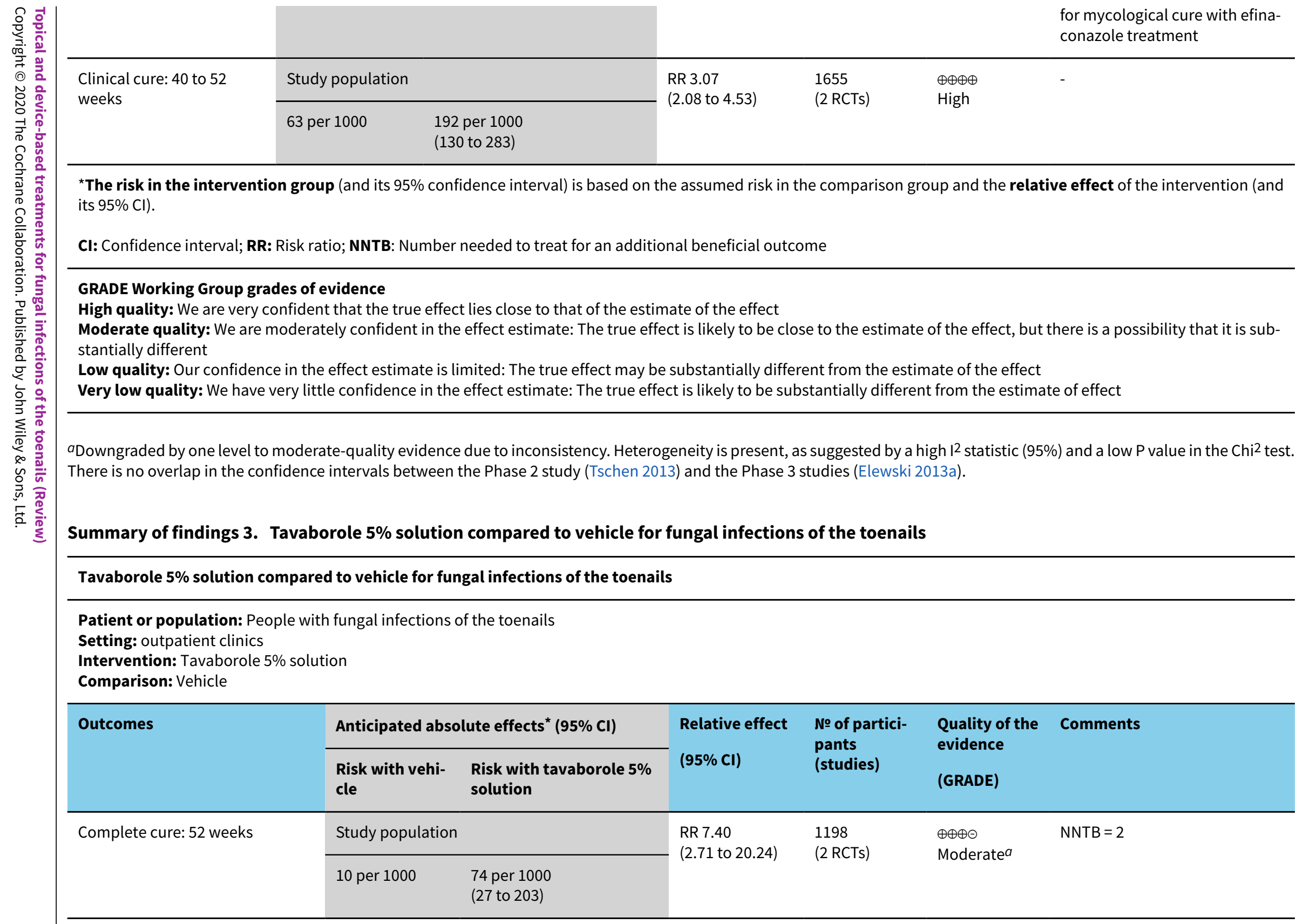

${ }^{\star}$ The risk in the intervention group (and its 95\% confidence interval) is based on the assumed risk in the comparison group and the relative effect of the intervention (and

effect lies close to that of the estimate of the effect

stantially different

a Downgraded by one level to moderate-quality evidence due to inconsistency. Heterogeneity is present, as suggested by a high $\mathrm{I}^{2}$ statistic ( $\left.95 \%\right)$ and a low $\mathrm{P}$ value in the Chi 2 test. There is no overlap in the confidence intervals between the Phase 2 study (Tschen 2013) and the Phase 3 studies (Elewski 2013a).

Summary of findings 3 . Tavaborole $5 \%$ solution compared to vehicle for fungal infections of the toenails 


\begin{tabular}{|c|c|c|c|c|c|c|c|}
\hline \multirow{2}{*}{\multicolumn{2}{|c|}{ 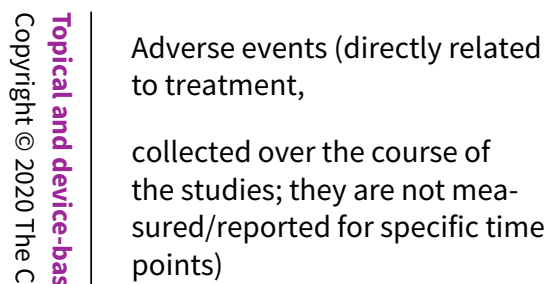 }} & \multicolumn{2}{|c|}{ Study population } & \multirow{2}{*}{$\begin{array}{l}\text { RR } 3.82 \\
\text { (1.65 to } 8.85 \text { ) }\end{array}$} & \multirow{2}{*}{$\begin{array}{l}1186 \\
\text { (2 RCTs) }\end{array}$} & \multirow{2}{*}{$\begin{array}{l}\oplus \oplus \oplus \ominus \\
\text { Moderate } a\end{array}$} & \multirow{2}{*}{$\begin{array}{l}\text { Application-site reactions were } \\
\text { the majority of reported ad- } \\
\text { verse events related to treat- } \\
\text { ment and included exfoliation, } \\
\text { dermatitis, erythema, and pain }\end{array}$} \\
\hline & & 15 per 1000 & $\begin{array}{l}58 \text { per } 1000 \\
(25 \text { to } 134)\end{array}$ & & & & \\
\hline 疍 & Mycological cure: 52 weeks & \multicolumn{2}{|c|}{ Study population } & \multirow{2}{*}{$\begin{array}{l}\text { RR } 3.40 \\
\text { (2.34 to } 4.93 \text { ) }\end{array}$} & \multirow{2}{*}{$\begin{array}{l}1198 \\
\text { (2 RCTs) }\end{array}$} & \multirow{2}{*}{$\begin{array}{l}\oplus \oplus \oplus \oplus \\
\text { High }\end{array}$} & \multirow[t]{2}{*}{ NNTB $=1$} \\
\hline 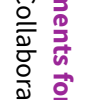 & & 98 per 1000 & $\begin{array}{l}332 \text { per } 1000 \\
\text { (229 to } 482 \text { ) }\end{array}$ & & & & \\
\hline $\overrightarrow{\underline{O}}$ & Clinical Cure - not measured & - & - & - & - & - & \\
\hline
\end{tabular}

${ }^{*}$ The risk in the intervention group (and its 95\% confidence interval) is based on the assumed risk in the comparison group and the relative effect of the intervention (and its $95 \% \mathrm{Cl})$.

Cl: Confidence interval; RR: Risk ratio; NNTB: Number needed to treat for an additional beneficial outcome

\section{GRADE Working Group grades of evidence}

High quality: We are very confident that the true effect lies close to that of the estimate of the effect

Moderate quality: We are moderately confident in the effect estimate: The true effect is likely to be close to the estimate of the effect, but there is a possibility that it is substantially different

Low quality: Our confidence in the effect estimate is limited: The true effect may be substantially different from the estimate of the effect

Very low quality: We have very little confidence in the effect estimate: The true effect is likely to be substantially different from the estimate of effect

aDowngraded by one level to moderate-quality evidence due to imprecision. Very large and variable confidence intervals across both studies, with low event rates (fewer than 100).

\section{Summary of findings 4. P-3051 (ciclopirox $8 \%$ hydrolacquer) compared to comparators for fungal infections of the toenails}

P-3051 (ciclopirox 8\% hydrolacquer) compared to comparators ${ }^{a}$ for fungal infections of the toenails

Patient or population: People with fungal infections of the toenails

Setting: outpatient clinics

Intervention: P-3051 (ciclopirox 8\% hydrolacquer)

Comparison: Comparators $a$

$\begin{array}{lllll}\text { Outcomes } & \text { Anticipated absolute effects* }(95 \% \mathrm{CI}) & \begin{array}{l}\text { Relative ef- } \\ \text { fect }\end{array} & \begin{array}{l}\text { No of partici- } \\ \text { pants }\end{array} & \begin{array}{l}\text { Quality of the } \\ \text { evidence }\end{array} \\ & & (95 \% \mathrm{CI}) & \text { (studies) } & \text { (GRADE) }\end{array}$




\begin{tabular}{|c|c|c|c|c|c|c|}
\hline & $\begin{array}{l}\text { Risk with Com } \\
\text { parators } a\end{array}$ & $\begin{array}{l}\text { Risk with P-3051 (ci- } \\
\text { clopirox } 8 \% \text { hydrolac- } \\
\text { quer) }\end{array}$ & & & & \\
\hline \multirow[t]{2}{*}{ Complete cure: 48 to 52 weeks } & Study populat & & \multirow{2}{*}{$\begin{array}{l}\text { RR } 2.43 \\
\text { (1.32 to } 4.48)\end{array}$} & \multirow{2}{*}{$\begin{array}{l}490 \\
\text { (2 RCTs) }\end{array}$} & \multirow{2}{*}{$\begin{array}{l}\oplus \oplus \oplus \ominus \\
\text { Moderate }\end{array}$} & \multirow[t]{2}{*}{ NNTB $=6$} \\
\hline & 52 per 1000 & $\begin{array}{l}127 \text { per } 1000 \\
(69 \text { to } 235)\end{array}$ & & & & \\
\hline \multirow{2}{*}{$\begin{array}{l}\text { Adverse events } \\
\text { (collected over the course of the } \\
\text { studies; they are not measured/re- } \\
\text { ported for specific time points) }\end{array}$} & \multicolumn{2}{|c|}{ Study population } & \multirow{2}{*}{$\begin{array}{l}\text { RR } 0.60 \\
\text { (0.19 to } 1.92)\end{array}$} & \multirow{2}{*}{$\begin{array}{l}487 \\
\text { (2 RCTs) }\end{array}$} & \multirow{2}{*}{$\begin{array}{l}\oplus \oplus \ominus \ominus \\
\text { Lowc }\end{array}$} & \multirow{2}{*}{$\begin{array}{l}\text { The most commonly report- } \\
\text { ed adverse events reported by } \\
\text { Baran } 2009 \text { included erythema, } \\
\text { redness, and burning, while the } \\
\text { one participant reporting an } \\
\text { adverse reaction in lorizzo } 2016 \\
\text { was unrelated to treatment } \\
\text { (foot trauma) }\end{array}$} \\
\hline & 121 per 1000 & $\begin{array}{l}73 \text { per } 1000 \\
\text { (23 to } 233)\end{array}$ & & & & \\
\hline \multirow[t]{2}{*}{ Mycological cure: 48 to 52 weeks } & \multicolumn{2}{|c|}{ Study population } & \multirow{2}{*}{$\begin{array}{l}\text { RR } 1.08 \\
(0.85 \text { to } 1.37)\end{array}$} & \multirow{2}{*}{$\begin{array}{l}490 \\
\text { (2 RCTs) }\end{array}$} & \multirow{2}{*}{$\begin{array}{l}\oplus \oplus \oplus \ominus \\
\text { Moderated }\end{array}$} & \multirow[t]{2}{*}{ NNTB $=13$} \\
\hline & 875 per 1000 & $\begin{array}{l}945 \text { per } 1000 \\
(744 \text { to } 1000)\end{array}$ & & & & \\
\hline Clinical cure - not measured & - & - & - & - & - & - \\
\hline
\end{tabular}

${ }^{\star}$ The risk in the intervention group (and its $95 \%$ confidence interval) is based on the assumed risk in the comparison group and the relative effect of the intervention (and its $95 \% \mathrm{Cl})$.

Cl: Confidence interval; RR: Risk ratio; NNTB: Number needed to treat for an additional beneficial outcome

\section{GRADE Working Group grades of evidence}

High quality: We are very confident that the true effect lies close to that of the estimate of the effect

Moderate quality: We are moderately confident in the effect estimate: The true effect is likely to be close to the estimate of the effect, but there is a possibility that it is substantially different

Low quality: Our confidence in the effect estimate is limited: The true effect may be substantially different from the estimate of the effect

Very low quality: We have very little confidence in the effect estimate: The true effect is likely to be substantially different from the estimate of effect

aComparators were ciclopirox $8 \%$ lacquer (Baran 2009) and amorolfine 5\% (Iorizzo 2016).

bDowngraded by one level to moderate-quality evidence due to imprecision, as very low event rates (fewer than 100).

CDowngraded by two levels to low-quality evidence: one level due to inconsistency as there is wide variation in the effect estimates of the two studies. Also downgraded by one level due to imprecision as the confidence interval is large with the upper and lower limits indicating a different effect of the intervention, and event rates are low.

dDowngraded by one level to moderate-quality evidence due to inconsistency, as there is no overlap in confidence intervals and the 12 and $\mathrm{Chi}^{2}$ statistics suggest evidence of high heterogeneity. 


\section{4-nm Nd:YAG laser compared to control for fungal infections of the toenails}

Patient or population: People with fungal infections of the toenails

Setting: outpatient clinics

Intervention: 1064-nm Nd:YAG laser

Comparison: no treatment or sham treatment

\begin{tabular}{|c|c|c|c|c|c|c|}
\hline \multirow[t]{2}{*}{ Outcomes } & \multicolumn{2}{|c|}{$\begin{array}{l}\text { Anticipated absolute effects }{ }^{\star}(95 \% \\
\mathrm{Cl})\end{array}$} & \multirow[t]{2}{*}{$\begin{array}{l}\text { Relative ef- } \\
\text { fect } \\
(95 \% \mathrm{CI})\end{array}$} & \multirow[t]{2}{*}{$\begin{array}{l}\text { № of partici- } \\
\text { pants } \\
\text { (studies) }\end{array}$} & \multirow[t]{2}{*}{$\begin{array}{l}\text { Quality of the } \\
\text { evidence } \\
\text { (GRADE) }\end{array}$} & \multirow[t]{2}{*}{ Comments } \\
\hline & $\begin{array}{l}\text { Risk with no } \\
\text { treatment }\end{array}$ & $\begin{array}{l}\text { Risk with 1064-nm } \\
\text { Nd:YAG laser }\end{array}$ & & & & \\
\hline Complete cure - not measured & - & - & - & - & - & - \\
\hline $\begin{array}{l}\text { Adverse events (collected over the } \\
\text { course of the studies; they are not } \\
\text { measured/reported for specific time } \\
\text { points) }\end{array}$ & See comment & See comment & $\begin{array}{l}\text { RR } 4.85 \\
(0.24 \text { to } \\
97.11)\end{array}$ & $\begin{array}{l}85 \\
(2 \text { RCTs })\end{array}$ & $\begin{array}{l}\oplus \odot \odot \odot \\
\text { Very low } a\end{array}$ & $\begin{array}{l}\text { No adverse effects were reported } \\
\text { with the intervention in the study, } \\
\text { rated at high risk of bias. }\end{array}$ \\
\hline \multirow[t]{2}{*}{ Mycological cure: 52 weeks } & \multicolumn{2}{|c|}{ Study population } & \multirow{2}{*}{$\begin{array}{l}\text { RR } 1.04 \\
\text { (0.59 to } 1.85)\end{array}$} & \multirow{2}{*}{$\begin{array}{l}85 \\
(2 \text { RCTs })\end{array}$} & \multirow{2}{*}{$\begin{array}{l}\oplus \oplus \odot \odot \\
\text { Lowc }\end{array}$} & \multirow{2}{*}{$\begin{array}{l}\text { No participants in one of the studies } \\
\text { achieved mycological cure. }\end{array}$} \\
\hline & 302 per 1000 & $\begin{array}{l}314 \text { per } 1000 \\
(178 \text { to } 559)\end{array}$ & & & & \\
\hline Clinical cure - not measured & - & - & - & - & - & - \\
\hline
\end{tabular}

${ }^{*}$ The risk in the intervention group (and its 95\% confidence interval) is based on the assumed risk in the comparison group and the relative effect of the intervention (and its $95 \% \mathrm{Cl}$ ).

\section{Cl: Confidence interval; RR: Risk ratio}

\section{GRADE Working Group grades of evidence}

High quality: We are very confident that the true effect lies close to that of the estimate of the effect

Moderate quality: We are moderately confident in the effect estimate: The true effect is likely to be close to the estimate of the effect, but there is a possibility that it is substantially different

Low quality: Our confidence in the effect estimate is limited: The true effect may be substantially different from the estimate of the effect

Very low quality: We have very little confidence in the effect estimate: The true effect is likely to be substantially different from the estimate of effect

aDowngraded by three levels to very low-quality evidence: one level due to risk of bias, as one of two studies that reported the outcome did not blind study personnel or participants and was rated at high risk of bias. Downgraded by one level due to inconsistency, as the effect estimate has wide variation with effects in both direction. Finally, 
downgraded by two levels due to imprecision, as the confidence interval is large, with the upper and lower limits indicating a different effect of the intervention, and contains both the null effect and appreciable harm. The sample size and event rates are low (fewer than 100).

bDowngraded by three levels to very low-quality evidence: one level due to risk of bias, as the one study available for this comparison was not a blinded study and thus had high risk of bias for blinding of participants, personnel, and outcome assessors. Downgraded by one level due to inconsistency, as the effect estimate has wide variation with effects in both directions. Finally, downgraded by two levels due to imprecision as there is only one study available for this comparison, the confidence interval is large with the upper and lower limits indicating a different effect of the intervention, and contains both the null effect and appreciable benefit. The sample size and event rates are low.

'Downgraded by two levels to low-quality evidence: one level due to risk of bias as one of the two studies that reported the outcome did not blind study personnel or participants

and was rated at high risk of bias. Also downgraded by one level due to imprecision, as there is a low number of events (fewer than 100) and a very low sample size.

\section{Summary of findings 6 . Luliconazole $5 \%$ solution compared to vehicle for fungal infections of the toenails}

\section{Luliconazole $5 \%$ solution compared to vehicle for fungal infections of the toenails}

Patient or population: People with fungal infections of the toenails

Setting: outpatient clinics

Intervention: Luliconazole $5 \%$ solution

Comparison: Vehicle

\begin{tabular}{|c|c|c|c|c|c|c|}
\hline \multirow[t]{2}{*}{ Outcomes } & \multicolumn{2}{|c|}{ Anticipated absolute effects ${ }^{\star}(95 \% \mathrm{Cl})$} & \multirow{2}{*}{$\begin{array}{l}\text { Relative effect } \\
(95 \% \mathrm{Cl})\end{array}$} & \multirow{2}{*}{$\begin{array}{l}\text { № of partici- } \\
\text { pants } \\
\text { (studies) }\end{array}$} & \multirow{2}{*}{$\begin{array}{l}\text { Quality of the } \\
\text { evidence } \\
\text { (GRADE) }\end{array}$} & \multirow[t]{2}{*}{ Comments } \\
\hline & $\begin{array}{l}\text { Risk with Vehi- } \\
\text { cle }\end{array}$ & $\begin{array}{l}\text { Risk with Luliconazole 5\% } \\
\text { solution }\end{array}$ & & & & \\
\hline \multirow[t]{2}{*}{ Complete cure: 48 weeks } & \multicolumn{2}{|l|}{ Study population } & \multirow{2}{*}{$\begin{array}{l}\text { RR } 2.96 \\
\text { (1.18 to } 7.41)\end{array}$} & \multirow{2}{*}{$\begin{array}{l}293 \\
(1 \mathrm{RCT})\end{array}$} & \multirow{2}{*}{$\begin{array}{l}\oplus \ominus \ominus \ominus \\
\text { Very lowa }\end{array}$} & \multirow[t]{2}{*}{ NNTB $=3$} \\
\hline & 51 per 1000 & $\begin{array}{l}149 \text { per } 1000 \\
(60 \text { to } 374)\end{array}$ & & & & \\
\hline \multirow{2}{*}{$\begin{array}{l}\text { Adverse events (collected over } \\
\text { the course of the studies; they } \\
\text { are not measured/reported for } \\
\text { specific time points) }\end{array}$} & \multicolumn{2}{|l|}{ Study population } & \multirow{2}{*}{$\begin{array}{l}\text { RR } 1.02 \\
\text { (0.90 to } 1.16)\end{array}$} & \multirow{2}{*}{$\begin{array}{l}293 \\
(1 \mathrm{RCT})\end{array}$} & \multirow{2}{*}{$\begin{array}{l}\oplus \oplus \ominus \ominus \\
\text { Low }^{b}\end{array}$} & \multirow{2}{*}{$\begin{array}{l}\text { Commonly-reported ad- } \\
\text { verse events included dry } \\
\text { skin, contact dermatitis, } \\
\text { paronychia, eczema, and } \\
\text { hyperkeratosis }\end{array}$} \\
\hline & 768 per 1000 & $\begin{array}{l}783 \text { per } 1000 \\
\text { (691 to } 891)\end{array}$ & & & & \\
\hline \multirow[t]{2}{*}{ Mycological cure: 48 weeks } & Study population & & \multirow{2}{*}{$\begin{array}{l}\text { RR } 1.39 \\
\text { (0.98 to } 1.97 \text { ) }\end{array}$} & \multirow{2}{*}{$\begin{array}{l}293 \\
(1 \mathrm{RCT})\end{array}$} & \multirow{2}{*}{$\begin{array}{l}\oplus \oplus \odot \ominus \\
\text { Lowb }\end{array}$} & \multirow[t]{2}{*}{ NNTB $=6$} \\
\hline & 293 per 1000 & $\begin{array}{l}407 \text { per } 1000 \\
\text { ( } 287 \text { to } 577)\end{array}$ & & & & \\
\hline Clinical Cure - not measured & - & - & - & - & - & - \\
\hline
\end{tabular}

${ }^{\star}$ The risk in the intervention group (and its 95\% confidence interval) is based on the assumed risk in the comparison group and the relative effect of the intervention (and its $95 \% \mathrm{Cl})$. 


\section{GRADE Working Group grades of evidence}

High quality: We are very confident that the true effect lies close to that of the estimate of the effect

Moderate quality: We are moderately confident in the effect estimate: The true effect is likely to be close to the estimate of the effect, but there is a possibility that it is sub-

stantially different

Low quality: Our confidence in the effect estimate is limited: The true effect may be substantially different from the estimate of the effect

Very low quality: We have very little confidence in the effect estimate: The true effect is likely to be substantially different from the estimate of effect

aDowngraded by three levels to very low-quality evidence. Downgraded by one level due to risk of bias as blinding of participants, personnel, and outcome assessors is unclear and there is high risk of reporting bias in the one study that is available for this comparison. Also downgraded by a further two levels due to imprecision, as there is only one study available for this comparison, the confidence interval is large, and sample size and event rates are low.

bDowngraded by two levels to low-quality evidence. Downgraded by one level due to risk of bias, as blinding of participants, personnel, and outcome assessors is unclear and there is high risk of reporting bias in the one study that is available for this comparison. Also downgraded by one level due to imprecision, as there is only one study available for this comparison, and sample size and event rates are low. 


\section{B A C K G R O U N D}

Please see Table 1 for a glossary of unfamiliar terms.

\section{Description of the condition}

Onychomycosis is a fungal infection of the nail apparatus (Zaias 1972). It is primarily caused by dermatophytes, which are infectious fungi that feed on the keratin present in the skin, hair, and nails, that usually cause an inflammatory response at the site of infection (Welsh 2010). Yeasts and non-dermatophyte moulds can also cause onychomycosis, either alone or in combination with dermatophytes (Gupta 2012; Welsh 2010). Fungi are ubiquitous in the environment and are found in greater abundance in warm or damp areas that foster growth (e.g. public change rooms and showers of athletic facilities, sweaty shoes) (Lipner 2018). For fungal infection to occur, a portal of entry to the nail unit has to exist; this usually occurs when there is trauma (i.e. injury) to the seal or space between the nail bed and nail plate (Figure 1) (Lipner 2018). Trauma (i.e. nail deformity) can also occur from long-term wear of ill-fitting or tight shoes (Lipner 2018). Many people seek treatment for cosmetic purposes, but onychomycosis is an infectious condition, so it is critical that it is treated to prevent cross-contamination to family members, and secondary complications such as tinea pedis (Szepietowski 2006). Patients with onychomycosis report decreased quality of life and may restrict their activities because of embarrassment about the aesthetic appearance of their nails (Reich 2011).

\section{Figure 1. Anatomy of the nail}

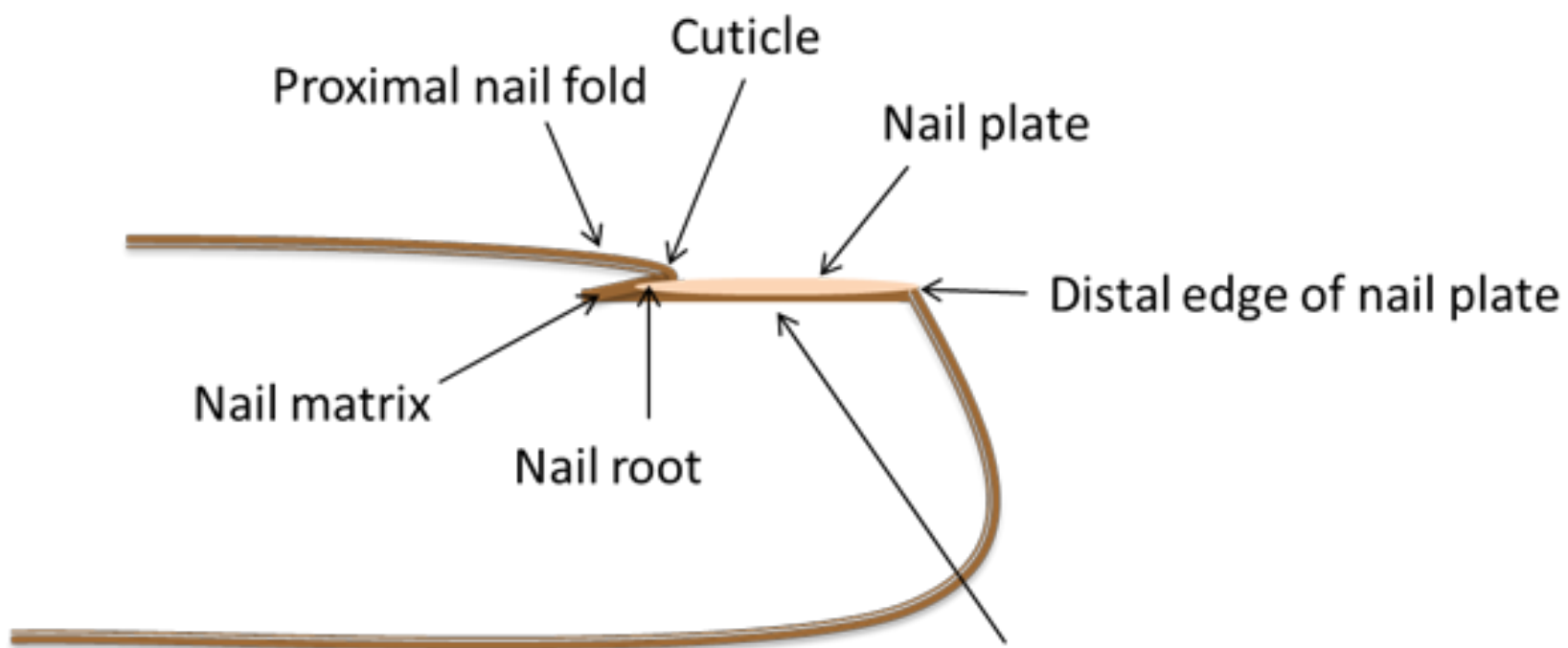

Nail bed

\section{Clinical presentation}

Onychomycosis has several clinical presentations, including distal and lateral subungual (under the nail) onychomycosis (DLSO), proximal subungual onychomycosis, superficial white onychomycosis, and total dystrophic onychomycosis (Hay 2011). DLSO is the most common form of onychomycosis and often presents as a result of tinea pedis (Hay 2011). DLSO affects the nail plate, nail bed, and hyponychium, which is the skin beneath the distal free edge of the nail, and may present with streaks of pigmentation in the nail and uneven borders, which distinguishes it from simple onycholysis, where the nail detaches from the nail bed (Hay 2011). Proximal subungual onychomycosis is initiated at the proximal nail fold and is usually whitish in colour, while superficial white onychomycosis is localised to the centre of the nail plate with a white, chalky appearance (Hay 2011). Total dystrophic onychomycosis is the most severe form of onychomycosis; it affects the entire nail plate and results in nail thickening along with complete loss of normal nail architecture (Hay 2011). The severity of onychomycosis can be described as mild, moderate, or severe. Published studies will assign a label of mild-moderate disease if less than approximately $60 \%$ of the nail is showing symptoms (i.e. discolouration). This upper limit is variable: studies will use values between $50 \%$ and $75 \%$ and use the term mild-moderate disease (Elewski 2013c; Elewski 2015a; Gupta 2000a; Iorizzo 2016). It is often at the clinician's discretion to determine what is mild-moderate and severe disease.

Figure 1 presents the anatomy of the nail, including the distal, lateral, and proximal areas of the nail.

\section{Epidemiology}

Onychomycosis accounts for approximately $50 \%$ of reported nail disorders (Faergemann 2003). A systematic review (Sigurgeirsson 2014) reported the population-based prevalence of onychomycosis as $4.3 \%$ in Europe and North America and found it was more prevalent in men in North America and Europe and in women in South America. The review reported that onychomycosis was more common in toenails than in fingernails, with clinic-based studies indicating toenail infections to be 10 times more common than fingernail infections. Infection was most commonly caused by the dermatophyte fungal organism Trichophyton rubrum (44.9\%), followed by yeasts (21.2\%), other dermatophytes (20.1\%), and moulds (13.3\%; Sigurgeirsson 2014). 


\section{Risk factors and comorbidities}

Onychomycosis is associated with a number of medical conditions and with age (Baran 2011). Patient characteristics such as advancing age, family members with tinea pedis or onychomycosis, genetics, and obesity may increase the risk of onychomycosis (Baran 2011; Rosen 2015). Some medical conditions occur concurrently with onychomycosis, such as diabetes, immunosuppression, peripheral vascular disease, and psoriasis, and can also increase risk for onychomycosis (Baran 2011; Döner 2011; Rosen 2015). There is an increased prevalence of onychomycosis in individuals with psoriasis, diabetes, immunosuppression, peripheral vascular disease, and obesity (Baran 2011; Döner 2011; Gupta 1997; Gupta 2000; Ozkan 2013). It is critical to treat and to monitor onychomycosis in individuals with these conditions, as secondary complications are a concern. Poor circulation, compromised immune systems, and inability to adequately care for the feet may exacerbate a fungal infection and provide opportunity for systemic fungal or bacterial infections to develop, with potential for cellulitis and septicaemia (Döner 2011; Fukunaga 2013; Gupta 2000; Ozkan 2013). Symptoms of psoriasis may worsen and treatment become complicated by the presence of fungus (Crowley 2015; Rigopoulos 2017; Ventura 2017). It is well documented in diabetes that complications with inadequate foot and nail care include foot ulcers and cellulitis, with progression to amputation and sometimes even death (Akkus 2017; Papini 2013; Rossaneis 2013). These patients may also have contraindications for the oral antifungals terbinafine and itraconazole due to drugdrug interactions or a higher risk of adverse events (Baran 2008; Barber 2006).

\section{Description of the intervention}

Topical drugs for onychomycosis generally come in the form of lacquers or solutions directly applied to the surface of the nail (Gupta 2013). Device-based therapies may include, but are not limited to, laser systems, iontophoresis, photodynamic therapy, and ultrasound (Gupta 2013). Topical drugs or devices are used in cases of mild-to-moderate onychomycosis and where oral antifungal drugs are contraindicated due to comorbidities or concerns with adverse events (Gupta 2014b). Oral treatments for fungal infections of the toenails are also commonly used (Gupta 2015a), but they are outside the scope of this review.

\section{How the intervention might work}

\section{Topical drugs}

Antifungal drugs can either be fungistatic, preventing further growth of fungal cells, or fungicidal, killing fungal cells entirely. Most antifungal drugs inhibit cell membrane synthesis, but others target protein translation and fungal proteases (Gupta 2013). Topical drugs are usually applied daily for 12 months in order to allow the normal nail to grow and replace the regions damaged by infection, although a few studies have used between six and 12 months (Gupta 2014b). Drugs formulated for topical application in onychomycosis include those from the allylamine (e.g. butenafine, terbinafine), azole (e.g. clotrimazole, efinaconazole, miconazole), hydroxypyridone (e.g. ciclopirox), morpholine-derivative (e.g. amorolfine, Kunzea oil), and benzoxaborole (e.g. tavaborole) classes (Gupta 2013; Gupta 2014a). The azoles, allylamines, and morpholine-derivative drugs inhibit ergosterol biosynthesis, an essential component of the cell wall (Gupta 1994). The hydroxypyridone-class drug ciclopirox inhibits metalloproteases by binding metal ions (metalloproteases are enzymes that help with fungal cell survival) (Valeant 2004). The benzoxaborole-class drugs inhibit protein translation by inhibiting the fungal leucine transfer ribonucleic acid (tRNA) synthetase (Rock 2007).

Application of topical treatments is usually daily for 12 months (Elewski 2013c; Elewski 2015a; Gupta 2000a), with amorolfine applied once or twice weekly for 12 months (Galderma 2011; Iorizzo 2016). Topical treatments come in cream, lacquer, and solutions of varying concentrations (e.g. $5 \%$ to $10 \%$ ) and are applied to the nail plate and skin surrounding the nail. In the case of lacquers, alcohol solution is used to remove buildup of lacquer on the nails; daily removal with amorolfine and weekly removal with ciclopirox is recommended (Dermik 2003; Galderma 2011). Debridement, i.e. removal of infected nail by trimming or filing, or both, by patients as required or monthly by healthcare professionals may be advised, particularly in the case of lacquers (Dermik 2003; Galderma 2011). For ciclopirox, a hydrolacquer solution (P-3051), based on a film-forming, water-soluble vehicle, has been formulated which eliminates the need for nail filing or alcohol removal (Baran 2009). Chemical removal or softening of the nails can be achieved using urea paste or creams with the aim of increasing the effectiveness of topical treatments that are used in combination or to allow easier removal of infected nail (Gupta 2014b; Lahfa 2013). Topical treatments do not generally have drug interactions, which is useful where patients are already taking multiple oral medications (Lipner 2019). Adverse events are usually related to skin reactions around the nail, such as rash, itching, or burning (Elewski 2013c; Elewski 2013d; Elewski 2015a; Elewski 2015b). The standard topical treatment will depend on geographical location; where there is more than one option, clinicians will use their expertise to determine treatment.

\section{Devices}

Devices have diverse mechanisms of action. Laser device systems are thought to act through selective photothermolysis, which is the conversion of light energy into heat energy that is confined to a specific target (Anderson 1983). The specific target in this case is fungi, which selectively absorb heat that leads to cell death (Gupta 2016b). Iontophoresis devices use an electrical current to increase the penetration of a topically-applied drug into the nail plate (Amichai 2010). Photodynamic therapy uses a narrow-spectrum light source to activate topically-applied photosensitisers (Piraccini 2008). The activation of the photosensitiser creates cellular damage that kills fungal cells (Piraccini 2008). Treatment with devices occurs in a clinical setting and may require weekly or monthly visits. As with topicals, devices do not interact with other medications, and if adverse events occur they are generally associated with transient pain or discomfort with laser treatment (Hollmig 2014; Karsai 2017; Lipner 2019). Devices are considered alternatives to antifungal treatments and in the USA are approved to improve visual appearance, not to cure the infection (Lipner 2019). This Cochrane Review did not identify any new devices with novel mechanisms of action.

\section{Why it is important to do this review}

A Cochrane Review on dermatophyte infections of the feet and nails was published in 2007 (Crawford 2007). Since then, a substantial amount of research on topical drugs and device-based therapies for the treatment of onychomycosis has emerged in the scientific literature, warranting a review exclusively on toenails. Topical 
drugs and devices are important therapeutic options for mildto-moderate onychomycosis. The advantage of effective topical therapies for onychomycosis is that they are not distributed throughout the body systemically, which may mean there is less likelihood of drug interactions or adverse events.

As these categories of therapies increase, it is important that healthcare professionals have accurate information about the optimal dosage and treatment regimens, so they can effectively treat patients with new drugs and devices.

The plans for this review were published as a protocol (Gupta 2016a).

\section{OB JECTIVES}

To assess the clinical and mycological effects of topical drugs and device-based therapies for onychomycosis.

\section{METHODS}

\section{Criteria for considering studies for this review}

\section{Types of studies}

We include randomised controlled trials (RCTs) of topical and device-based therapies for onychomycosis. We exclude cross-over trials. This is because cross-over trials are only appropriate when the intervention does not produce a permanent change in patient status. The endpoint with onychomycosis treatment is cure, and a cross-over following cure would not be appropriate.

\section{Types of participants}

We include men and women over 18 years of age who have onychomycosis of the toenails. Infection was diagnosed based on studying nail samples using direct microscopic examination (potassium hydroxide), culture, periodic acid-Schiff stain (PAS), or molecular biology (e.g. polymerase chain reaction (PCR)). Some studies may have reported results of fingernails and toenails combined; in these cases, we tried to obtain results for toenails alone and when this was not possible, we excluded the study.

\section{Types of interventions}

We include RCTs that compared a topical or device-based therapy with placebo, vehicle, no treatment, or an active comparator that was topical or device-based.

\section{Types of outcome measures}

We assessed outcomes based on the following time points: less than 12 weeks, between 12 and 36 weeks, between 36 and 52 weeks, and more than 52 weeks. We excluded studies lasting less than 12 weeks. Our primary outcomes are presented in a 'Summary of findings' table.

\section{Primary outcomes}

- Complete cure rate: the proportion of participants with $0 \%$ nail plate involvement and mycological cure at follow-up.

- Adverse events: the proportion of participants who reported at least one event, whether or not related to the intervention. In addition and where possible, we reported adverse events based on the likelihood of their being related to the interventions, as judged by the study investigator(s).

\section{Secondary outcomes}

- Mycological cure rate: the proportion of participants who achieved negative mycological testing as defined by the study at follow-up.

- Clinical cure rate: the proportion of participants who achieved $0 \%$ nail plate involvement at follow-up.

- Compliance: the proportion of participants who completed the study protocol without deviations, as defined by study investigators.

- Time to recurrence.

- Quality of life: this was described based on measures included by study investigators.

- Pharmaco-economics: the cost of the treatment was described.

\section{Search methods for identification of studies}

We aimed to identify all relevant RCTs, regardless of language or publication status (published, unpublished, in press, or in progress).

\section{Electronic searches}

The Cochrane Skin Information Specialist searched the following databases up to 7 May 2019:

- the Cochrane Skin Group Specialised Register, using the search strategy in Appendix 1;

- the Cochrane Central Register of Controlled Trials (CENTRAL) 2019, Issue 5, in the Cochrane Library, using the strategy in Appendix 2;

- MEDLINE via Ovid (from 1946), using the strategy in Appendix 3;

- Embase via Ovid (from 1974), using the strategy in Appendix 4; and

- Latin American and Caribbean Health Science Information database (LILACS), from 1982, using the strategy in Appendix 5.

\section{Trial registries}

We (KF, RM) searched the following trials registers up to 13 May 2019 using the following terms: onychomycosis or tinea unguium.

- the ISRCTN registry (www.controlled-trials.com);

- ClinicalTrials.gov (www.clinicaltrials.gov);

- the Australian New Zealand Clinical Trials Registry (www.anzctr.org.au);

- the World Health Organization International Clinical Trials Registry platform (ICTRP) (apps.who.int/trialsearch); and

- the EU Clinical Trials Register (www.clinicaltrialsregister.eu).

\section{Searching other resources}

\section{References from published papers}

We checked the bibliographies of included and excluded studies for additional references to relevant RCTs.

We handsearched the Mycoses journal from 1957 to 1990 for additional studies. Later editions of the journal are indexed in MEDLINE and Embase, so it was not necessary to handsearch them. 


\section{Unpublished literature}

We conducted online searches of pharmaceutical company websites and the US Food and Drug Administration (FDA) website.

We contacted experts in the field of dermatology seeking information about unpublished trials, including those listed in trial registries and conference abstracts.

\section{Conference proceedings}

We handsearched the following conference proceedings for relevant trials for years that the Cochrane Skin Group had not already handsearched:

- British Association of Dermatologists (1982 to 2013);

- Annual Meetings of the American Academy of Dermatology (1990 to 2013);

- European Academy of Dermatology and Venereology (1996 to 2013);

- European Society for Dermatological Research (1970 to 2012); and

- Australasian College of Dermatologists (1996 to 2013).

\section{Adverse effects}

We did not perform a separate search for adverse effects of the target intervention. However, we examined data on adverse effects from the included studies that we identified.

\section{Data collection and analysis}

\section{Selection of studies}

We managed studies using Microsoft Excel 2010 software. Two review authors, KF and SV, screened the references and each performed independent study selection. We piloted the study selection criteria on a subsample of the articles (approximately 10 ) in order to validate the criteria and ensure that they were consistently interpreted for all studies. We examined all articles and documents retrieved to determine if they complied with the inclusion and exclusion criteria. We discussed disagreements in order to reach a consensus. If we could not reach consensus, we consulted a third review author (RM).

\section{Data extraction and management}

Two review authors (from KF, RM, or SV) independently extracted the data for each included study using a standard form, including the study design, number of participants randomised into each treatment group, baseline characteristics, health intervention, treatment regimen and duration, treatment success and failure, safety, tolerability, the number of participants lost to follow-up, the duration of follow-up, and pharmaco-economic data. We piloted the forms on a small group of studies to ensure completeness. We discussed disagreements in order to reach a consensus. If we could not reach consensus, a third review author performed additional data extraction. The first review author (KF) entered the data into Review Manager 5 (Review Manager 2014), and the second review author (RM or SV) verified the data. We were not blinded to authors, institutions, or journals during data extraction.

\section{Assessment of risk of bias in included studies}

Two authors, KF and RM, independently evaluated risks of bias in individual studies using Cochrane's 'Risk of bias' tool
(Higgins 2017), resolving disagreements by discussion. The 'Risk of bias' ('RoB') table includes the following domains: sequence generation, allocation concealment, blinding of participants and personnel, blinding of outcome assessment, incomplete outcome data, within-study selective outcome reporting, and other potential sources of bias (e.g. reporting outcomes for subgroups rather than all participants; reporting data for each group to which participants were randomised in a multiple-arm intervention study, rather than combining multiple arms).

\section{Measures of treatment effect}

We analysed dichotomous outcomes using a risk ratio (RR) with a 95\% confidence interval $(\mathrm{Cl})$.

We quantified significantly different outcomes as the number needed to treat for an additional beneficial/harmful outcome(NNTB/H), based on the following formula: NT = 1/ARC * (1-RR) (Centre for Evidence-Based Medicine). We used the RRs from the meta-analysis and the moderate assumed control risk (ARC) calculated in GRADEpro GDT (Guideline Development Tool) (GRADEPro).

\section{Unit of analysis issues}

The participant was the unit of analysis, with clinical trials designating one target toenail per participant as the unit of analysis. We did not consider nail data in clinical studies that used individual nails as the unit of analysis, including multiple toenails per participant in their data set. The exception to this was in the case of studies where multiple toenails per participant were included if outcomes were reported per participant. We did not consider participant-controlled data. We excluded withinparticipant designs because onychomycosis is contagious and can spread to other nails in the same foot of a single patient or between feet in a single patient. Furthermore, the effect of the intervention may also carry. Between-participant designs control this by treating all nails with the same intervention. We excluded cross-over trials with the aim of any treatment for onychomycosis as cure, which is a permanent change in patient status. Where studies had multiple intervention arms, all arms are mentioned in the Characteristics of included studies table.

\section{Dealing with missing data}

If data were missing or in a format that precluded extraction, we contacted the study authors to request that information. We prioritised intention-to-treat (ITT) data and converted per-protocol data into ITT data wherever possible (e.g. when attrition data were available). We considered participants who discontinued a study to be treatment failures. If we were unable to acquire all missing data, we performed analyses with the available data.

\section{Assessment of heterogeneity}

We assessed the heterogeneity of the included studies for clinical and statistical factors. Clinical factors may include population age, comorbidities, and disease severity. We quantified statistical heterogeneity using the 12 statistic (Higgins 2019). The Handbook provides a range of values for moderate (30\% to $60 \%)$, substantial ( $50 \%$ to $90 \%)$, and considerable $(75 \%$ to $100 \%)$ heterogeneity. 


\section{Assessment of reporting biases}

We planned to investigate selective reporting of outcomes in analyses with 10 studies or more (Dwan 2008), generating funnel plots of the data to determine if reporting asymmetry was present. We did not do this, as none of our analyses had 10 studies or more.

\section{Data synthesis}

We performed data synthesis using Review Manager 5 (Review Manager 2014). We calculated risk ratios (dichotomous data) using the Mantel-Haenszel model. Where meta-analysis was possible (e.g. at least two trials), we conducted the analyses using a randomeffects model. For studies with multiple intervention arms, we selected the relevant interventions. Where possible, we combined intervention arms so that we could include a single comparison in the meta-analysis. When this was not possible, we used the relevant intervention arms in the analysis and identified them in the footnotes of the forest plot. Where we estimated results for individual studies with low numbers of events (fewer than 10 in total) or where the total sample size was fewer than 30 participants and we used a risk ratio, we reported the proportion of events in each group together with a P value from a Fisher's Exact test.

If meta-analysis was not possible due to the number of trials (fewer than two), we assessed the studies for the data collected qualitatively.

\section{Subgroup analysis and investigation of heterogeneity}

When we found moderate statistical heterogeneity ( 12 statistic greater than 50\%), we conducted subgroup analysis. We analysed any clinical sources of heterogeneity, such as participant age and onychomycosis severity, if we had sufficient studies (at least two studies with a combined sample size of 30 or more for each group being compared (Dijkman 2009)).

\section{Sensitivity analysis}

In analyses with considerable heterogeneity (12 statistic greater than $75 \%$ ), we conducted a sensitivity analysis to determine if removing studies with identified risks of bias contributed to the overall heterogeneity.

\section{'Summary of findings' tables}

We present the data for the primary outcomes (complete cure and adverse events) and for two of our secondary outcomes (mycological cure and clinical cure) in six 'Summary of findings' tables. We assessed the quality of the evidence across the outcome measures that were reported in the 'Summary of findings' tables using the GRADE assessment and GRADEpro GDT software (GRADEPro; Ryan 2016a; Ryan 2016b). The comparisons for which we created tables were: ciclopirox $8 \%$ lacquer versus vehicle; P-3051 (ciclopirox 8\% hydrolacquer) versus comparators; efinaconazole $10 \%$ solution versus vehicle; tavaborole $5 \%$ solution versus vehicle; luliconazole $5 \%$ solution versus vehicle; $1064-\mathrm{nm}$ $\mathrm{Nd}$ :YAG laser versus control.

\section{RES U L T S}

\section{Description of studies}

\section{Results of the search}

Searches of electronic databases, clinical trial registries, and conference proceedings yielded 1745 records. We found an additional 23 records from checking the reference lists of included and excluded studies. After removing duplicates, we had 1402 unique records for screening. We excluded 1259 records based on title/abstract review. We assessed the full text of 143 records for eligibility. We excluded 51 records (Characteristics of excluded studies). We identified 13 ongoing studies (see Characteristics of ongoing studies) and 22 studies awaiting classification (see Characteristics of studies awaiting classification).

We included 56 studies reported in 57 references (see Characteristics of included studies). We included 12 of these studies in the meta-analysis, and described the results of the remaining 44. Of these 44 studies, seven were published recently (2014 to 2019) and are depicted individually using forest plots. For a further description of our screening process, see the study flow diagram (Figure 2). We contacted authors for 48 of the 56 included studies and received a response, positive or negative, for 29 of the 48 studies (Table 2). 
Figure 2. Study flow diagram.

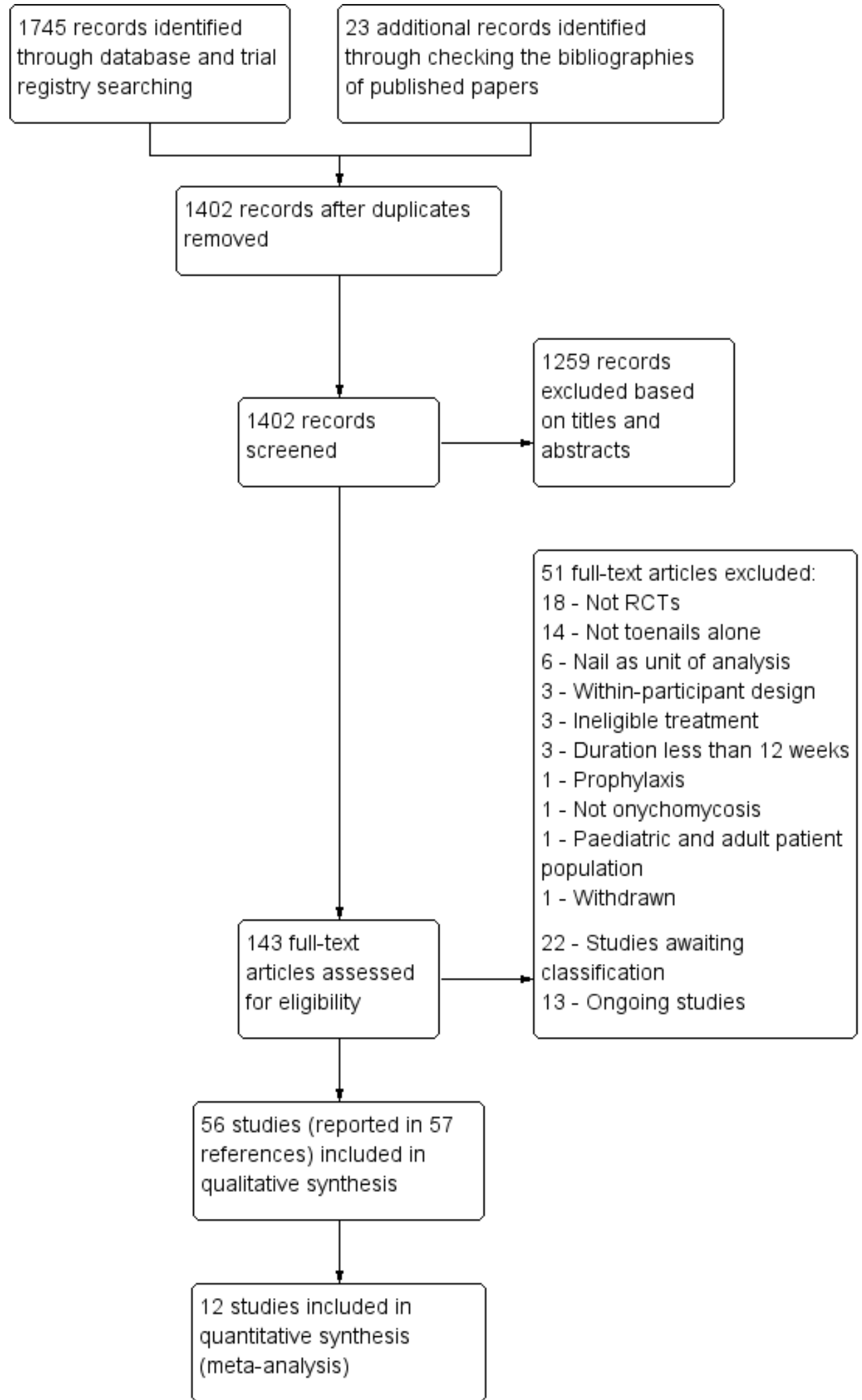




\section{Included studies}

\section{Trial settings}

All 56 studies were RCTs, with a total of 12,501 participants. Twenty-four studies were described as multicentre. Twenty-two studies were published in $\mathbf{2 0 1 0}$ or earlier. Forty-three studies were conducted in outpatient dermatology settings; 23 studies had at least one trial site in the USA and 18 studies had a European trial site.

\section{Trial design}

Thirty-nine studies were two-arm studies (ACTRN12614000946617; ACTRN12614001238662; Alberdi 2019; Amichai 2010; Bonhert 2019; Buck 1994; Eertmans 2018; Elewski 2013b; Elewski 2013c; Elewski 2013d; Elewski 2015a; Elewski 2015b; EUCTR2006-005895-42; EUCTR2008-003215-13; Gupta 2000a; Gupta 2000b; Hollmig 2014; Iorizzo 2016; Karsai 2017; Koren 2018; Kumar 2001; Lahfa 2013; Montana 1994; NCT01080079; NCT01246518; NCT01400594; NCT02343627; NCT03141840; NCT03289871; Nijenhuis-Rosien 2019; Parekh 2017; Paul 2013; Romero-Cerecero 2008; RomeroCerecero 2009; Schalka 2012; Sigurgeirsson 2016; Syed 1998; Syed 1999; Watanabe 2017).

The remaining 17 studies had three arms or more (Auvinen 2015; Baran 2009; Elewski 2013a; EUCTR2005-005905-51; EUCTR2006-000974-54; EUCTR2016-001242-25; Gupta 2006; Hartmane 2013; ljzerman 2010; Lu 2005; NCT01145807; NCT02022215; NCT02933879; Stein 2014; Toledo-Bahena 2014; Tschen 2013; Waibel 2013). All interventions are described in the Characteristics of included studies tables. For studies with multiple intervention arms that were included in meta-analyses, the relevant interventions were identified and used in the analysis. For example, Tschen 2013 includes three efinaconazole arms; in our meta-analysis, we used the efinaconazole arm that was consistent with that assessed in the Phase 3, two-arm studies (Elewski 2013c; Elewski 2013d) and thus the approved treatment for patient use. Treatments used in meta-analyses are identified in the footnotes of forest plots. For single studies, we list all interventions and available results in Table 3. All topical treatment studies used one target toenail per person, usually the big toenail, to evaluate treatment. Device trials evaluated multiple nails per person; however, we were able to include the studies because treatment outcomes were reported per person, so all affected nails had to be cured for a person to be considered cured (Hollmig 2014; Karsai 2017; Waibel 2013).

\section{Participants}

Sample sizes varied from 10 to 1044 participants (median: 119). The age of the participants across studies ranged from 27 to 68 years of age. As per eligibility criteria, all participants were aged 18 and over. All studies, with the exception of Gupta 2006 and Lu 2005, specified the inclusion of both genders. With the exception of one study, in which a specific patient group was used (Chinese participants with onychomycosis) (Gupta 2006), all studies were open to general dermatology patients diagnosed with onychomycosis. Approximately $63 \%$ (35 studies) specifically examined onychomycosis caused by dermatophytes alone, requiring confirmation of dermatophyte infection prior to study entry (Amichai 2010; Auvinen 2015; Baran 2009; Buck 1994; Elewski 2013a; Elewski 2013b; Elewski 2015a; Elewski 2015b; EUCTR2005-005905-51;
EUCTR2006-000974-54; EUCTR2006-005895-42; Gupta 2000a; Gupta 2000b; Gupta 2006; Hartmane 2013; Ijzerman 2010; Karsai 2017; Koren 2018; Kumar 2001; Lahfa 2013; Lu 2005; NCT01080079; NCT01246518; NCT01400594; NCT02022215; NCT02343627; NCT02933879; NCT03141840; Parekh 2017; Paul 2013; Romero-Cerecero 2008; Syed 1998; Syed 1999; ToledoBahena 2014; Watanabe 2017). Nine studies included infections caused by yeasts, either alone or in combination with dermatophytes (Alberdi 2019; Bonhert 2019; Eertmans 2018; Elewski 2013c; Elewski 2013d; Iorizzo 2016; Schalka 2012; Sigurgeirsson 2016; Tschen 2013), and three studies included non-dermatophyte moulds in combination with dermatophytes (Hollmig 2014; NCT01145807; Schalka 2012), two studies included non-dermatophyte infections alone (Alberdi 2019; Iorizzo 2016), and 11 studies did not clearly specify (ACTRN12614000946617; ACTRN12614001238662; Eertmans 2018 EUCTR2008-003215-13; EUCTR2016-001242-25; Montana 1994; NCT03289871; NijenhuisRosien 2019; Romero-Cerecero 2009; Stein 2014; Waibel 2013). Where reported, most participants had more than one toenail affected by onychomycosis, with nine studies reporting an average number of toenails affected of four or more (Baran 2009; Elewski 2013a; Elewski 2013b; EUCTR2006-000974-54; Iorizzo 2016; Nijenhuis-Rosien 2019; Romero-Cerecero 2009; Sigurgeirsson 2016; Tschen 2013) and four studies reporting an average number of toenails affected of fewer than four (Elewski 2013c; Elewski 2013d; Elewski 2015a; Elewski 2015b). Two studies used the number of toenails affected as an exclusion criterion: for EUCTR2008-003215-13 no more than three affected toenails per person were allowed, and for Lahfa 2013 no more than two toenails were allowed.

Most studies did not report the disease duration of the participants enrolled (ACTRN12614000946617; ACTRN12614001238662; Amichai 2010; Baran 2009; Bonhert 2019; Eertmans 2018; Elewski 2013a; Elewski 2013b; Elewski 2013c; Elewski 2013d; Elewski 2015a; Elewski 2015b; EUCTR2005-005905-51; EUCTR2006-005895-42; EUCTR2008-003215-13; EUCTR2016-001242-25; Gupta 2006; Hartmane 2013; Hollmig 2014; Ijzerman 2010; lorizzo 2016; Karsai 2017; Kumar 2001; Lu 2005; Montana 1994; NCT01080079; NCT01145807; NCT01246518; NCT01400594; NCT02022215; NCT02343627; NCT02933879; NCT03141840; NCT03289871; Nijenhuis-Rosien 2019; Parekh 2017; Paul 2013; Romero-Cerecero 2009; Stein 2014; Toledo-Bahena 2014; Tschen 2013; Waibel 2013; Watanabe 2017). In fact, only 13 of 56 studies reported disease duration in some way (Alberdi 2019; Auvinen 2015; Buck 1994; EUCTR2006-000974-54; Gupta 2000a; Gupta 2000b; Koren 2018; Lahfa 2013; Romero-Cerecero 2008; Schalka 2012; Sigurgeirsson 2016; Syed 1998; Syed 1999). Many participants had a disease duration of five years or longer in Auvinen 2015 (75\%), EUCTR2006-000974-54 (mean seven years), Gupta 2000a and Gupta 2000b (group means ranging from 10.8 to 11.8 years), Koren 2018 (mean 11.7 years), Romero-Cerecero 2008 (29\%), and Sigurgeirsson 2016 (62\%), while shorter disease durations of a few months to three years were reported in Buck 1994; Schalka 2012; Syed 1998; and Syed 1999; lastly, Lahfa 2013 reported mean disease durations of 4.1 and 6.3 years, whlie Alberdi 2019 reported a disease duration of three to five years.

Matrix/lunula involvement was specified as excluded in 23 studies (ACTRN12614001238662; Amichai 2010; Eertmans 2018; Elewski 2013a; Elewski 2013b; Elewski 2013c; Elewski 2013d; EUCTR2006-000974-54; Hartmane 2013; Ijzerman 2010; Iorizzo 
2016; NCT01080079; NCT01145807; NCT02343627; NCT02933879; NCT03141840; NCT03289871; Paul 2013; Romero-Cerecero 2008; Schalka 2012; Sigurgeirsson 2016; Tschen 2013; Watanabe 2017), while 33 studies did not specify whether matrix involvement was included or excluded (ACTRN12614000946617; Alberdi 2019; Auvinen 2015; Baran 2009; Bonhert 2019; Buck 1994; Elewski 2015a; Elewski 2015b; EUCTR2005-005905-51; EUCTR2006-005895-42; EUCTR2008-003215-13; EUCTR2016-001242-25; Gupta 2000a; Gupta 2000b; Gupta 2006; Hollmig 2014; Karsai 2017; Koren 2018; Kumar 2001; Lahfa 2013; Lu 2005; Montana 1994; NCT01246518; NCT01400594; NCT02022215; Nijenhuis-Rosien 2019; Parekh 2017; Romero-Cerecero 2009; Stein 2014; Syed 1998; Syed 1999; ToledoBahena 2014; Waibel 2013).

Forty studies enrolled participants with mild to moderate onychomycosis (ACTRN12614000946617; ACTRN12614001238662; Amichai 2010; Baran 2009; Eertmans 2018; Elewski 2013a; Elewski 2013b; Elewski 2013c; Elewski 2013d; Elewski 2015a; Elewski 2015b; EUCTR2005-005905-51; EUCTR2006-005895-42; EUCTR2008-003215-13; EUCTR2016-001242-25; Gupta 2000a; Gupta 2000b; Gupta 2006; Hartmane 2013; Ijzerman 2010; Iorizzo 2016; Lahfa 2013; Montana 1994; NCT01080079; NCT01145807; NCT01246518; NCT02022215; NCT02343627; NCT02933879; NCT03141840; NCT03289871; Paul 2013; Romero-Cerecero 2008; Romero-Cerecero 2009; Schalka 2012; Sigurgeirsson 2016; Stein 2014; Toledo-Bahena 2014; Tschen 2013; Watanabe 2017), while 12 studies did not specify severity (Auvinen 2015; Bonhert 2019; Buck 1994; EUCTR2006-000974-54; Karsai 2017; Koren 2018; Lu 2005; NCT01400594; Nijenhuis-Rosien 2019; Syed 1998; Syed 1999; Waibel 2013), one study enrolled severe onychomycosis (Parekh 2017), and three studies did not exclude participants based on disease severity (Alberdi 2019; Hollmig 2014; Kumar 2001).

\section{Interventions}

Trials evaluated several topical antifungal treatments including, but not limited to, topical solutions (efinaconazole, luliconazole, tavaborole and terbinafine $\mathrm{HCl}$ ), lacquers (ciclopirox, amorolfine, ciclopirox $8 \%$ hydrolacquer, terbinafine), creams (butenafine hydrochloride, bifonazole), devices (1064-nm neodymium-garnet lasers (1064-nm Nd:YAG)), and a combination thereof.

\section{Classes of drugs}

Classes of drugs were included as follows:

- 13 studies included allylamines (Amichai 2010; Elewski 2013a; Elewski 2013b; EUCTR2016-001242-25; Gupta 2006; Hartmane 2013; Lu 2005; NCT01080079; NCT01145807; NCT01246518; NCT01400594; Syed 1998; Syed 1999);

- 9 studies included azoles (Bonhert 2019; Buck 1994; Elewski 2013c; Elewski 2013d; EUCTR2008-003215-13; Lahfa 2013; Stein 2014; Tschen 2013; Watanabe 2017);

- 3 studies included benzoxaboroles (Elewski 2015a; Elewski 2015b; Toledo-Bahena 2014);

- 11 studies included hydroxypyridones (Baran 2009; EUCTR2005-005905-51; EUCTR2006-005895-42; Gupta 2000a; Gupta 2000b; Gupta 2006; Iorizzo 2016; Lu 2005; Paul 2013; Romero-Cerecero 2008; Schalka 2012); and

- 8 included morpholine-derivatives (ACTRN12614000946617; Auvinen 2015; Eertmans 2018; Elewski 2013b; EUCTR2006-000974-54; Koren 2018; NCT03289871; Sigurgeirsson 2016).

\section{Comparator arm}

- 11 studies had a placebo arm (ACTRN12614001238662; Kumar 2001; NCT01080079; NCT01400594; NCT02343627; NCT02933879; NCT03141840; Nijenhuis-Rosien 2019; Parekh 2017; Syed 1998; Syed 1999);

- 11 studies had a vehicle arm (Elewski 2013a; Elewski 2013c; Elewski 2013d; Elewski 2015a; Elewski 2015b; EUCTR2016-001242-25; Gupta 2000a; Gupta 2000b; Montana 1994; Stein 2014; Watanabe 2017);

- 2 studies used no treatment as a control (Hollmig 2014; Karsai 2017); and

- 22 studies used an active comparator (ACTRN12614000946617; ACTRN12614001238662; Alberdi 2019; Amichai 2010; Auvinen 2015; Bonhert 2019; Buck 1994; Eertmans 2018; Elewski 2013b; EUCTR2008-003215-13; EUCTR2016-001242-25; Iorizzo 2016; Koren 2018; Lahfa 2013; NCT01246518; NCT03289871; Paul 2013; Romero-Cerecero 2008; Romero-Cerecero 2009; Schalka 2012; Sigurgeirsson 2016; Waibel 2013).

Thirteen studies had multiple comparison groups; 11 of these studies had a vehicle/placebo arm and active comparator(s) (Baran 2009; EUCTR2005-005905-51; EUCTR2006-000974-54; EUCTR2016-001242-25; Gupta 2006; Ijzerman 2010; Lu 2005; NCT01145807; NCT02022215; Toledo-Bahena 2014; Tschen 2013) and the remaining two studies only had active comparators (Hartmane 2013; Waibel 2013).

\section{Intervention arm}

- 3 studies compared efinaconazole $10 \%$ solution to vehicle (Elewski 2013c; Elewski 2013d; Tschen 2013);

- 3 studies compared tavaborole $5 \%$ solution to vehicle (Elewski 2015a; Elewski 2015b; Toledo-Bahena 2014);

- 2 studies compared ciclopirox $8 \%$ hydrolacquer to comparator lacquers such as ciclopirox $8 \%$ and amorolfine 5\% (Baran 2009; lorizzo 2016);

- 2 studies compared ciclopirox $8 \%$ lacquer to vehicle (Gupta 2000a; Gupta 2000b);

- 1 study each evaluated different ciclopirox $8 \%$ lacquer regimens (Schalka 2012) and ciclopirox $8 \%$ hydrolacquer concentrations (Hartmane 2013); and

- 2 studies compared luliconazole solution with vehicle (Stein 2014; Watanabe 2017).

Amorolfine lacquer was often used as a comparator, to other topical lacquers such as Abicin 30\% nail lacquer (Auvinen 2015), to topical solutions such as terbinafine $\mathrm{HCl}$ (Elewski 2013b) and Kunzea oil (ACTRN12614000946617), to photodynamic therapy (PDT) (Koren 2018), and to sequential therapy of urea ointment and ciclopirox (Paul 2013).

Other treatments such as mastic paint (ACTRN12614001238662), NVXT solution (NCT02933879), and ABL01 (NCT03141840) were compared to placebo.

Device-based therapies included three studies comparing 1064$\mathrm{nm} \mathrm{Nd}$ :YAG laser monotherapy to an untreated group (Hollmig 2014; Karsai 2017) or sham control group (Nijenhuis-Rosien 2019), one study comparing different laser treatments (Waibel 2013), one study drilling holes into nails to facilitate drug delivery (EUCTR2006-005895-42), two studies using photodynamic therapy 
(PDT) (Alberdi 2019; Koren 2018), two studies using iontophoresis (Amichai 2010; NCT01080079), and one study using intense pulsed light (IPL) therapy (Alberdi 2019).

One study investigated amorolfine lacquer while using nail varnish (Sigurgeirsson 2016).

Study duration ranged from 12 weeks to 52 weeks, with most studies 48 to 52 weeks in length.

\section{Frequency}

The frequency with which topical treatments were applied varied, with the most common regimen being once daily for 36 to 48 weeks (22 studies: Auvinen 2015; Baran 2009; Bonhert 2019; Elewski 2013a; Elewski 2013b; Elewski 2013c; Elewski 2013d; Elewski 2015a; Elewski 2015b; EUCTR2006-005895-42; Gupta 2000a; Gupta 2000b; Ijzerman 2010; Iorizzo 2016; Montana 1994; NCT01246518; NCT01400594; NCT02022215; Paul 2013; Stein 2014; Tschen 2013; Watanabe 2017; two additional studies twice daily ACTRN12614000946617; NCT01145807). One study specified application for 48 weeks without frequency (EUCTR2016-001242-25). Daily application was common among other treatment durations as 10 studies adopted it (Amichai 2010; EUCTR2008-003215-13; NCT02343627; Syed 1999 (three times daily) for four to eight weeks; EUCTR2005-005905-51; Gupta 2006; Lahfa 2013; Lu 2005 for three months; Eertmans 2018; Hartmane 2013 for six months). Twice-daily application for other durations was used in three studies: Buck 1994 for six months; Kumar 2001 for three months; Syed 1998 for seven days. Alternate frequencies of topical application were: four times daily for one month (ACTRN12614001238662), twice daily for three months, then daily for three months (Parekh 2017), weekly for three months (Sigurgeirsson 2016) or six to 12 months (NCT03141840), daily for two weeks, then weekly to six months (EUCTR2006-000974-54), daily for three months, then three times a week for three months (Toledo-Bahena 2014), daily for two eight-week periods separated by 32 weeks (NCT02933879), and a six-month application with a first month of three times a week, a second month of twice a week, and the last four months of weekly application (Romero-Cerecero 2008; Schalka 2012).

Device-based treatments occurred weekly (Waibel 2013) or every other week (Hollmig 2014; Nijenhuis-Rosien 2019 (with an additional treatment at week 12)) for three to four weeks, every other week for 16 weeks (Alberdi 2019), weekly every four to six weeks (Karsai 2017), six treatments with one every three weeks (Koren 2018), or twice daily (NCT03289871) for six months, while NCT01080079 and Romero-Cerecero 2009 did not specify frequency of application.

\section{Co-interventions}

Co-interventions were infrequent, with six studies using debriding/ filing with topical treatments; Baran 2009, Gupta 2000a and Gupta $2000 \mathrm{~b}$ used filing with ciclopirox $8 \%$ lacquer treatment, Elewski 2013a and Elewski 2013b used filing with amorolfine 5\% lacquer treatment, and Buck 1994 used debridement with tea tree oil and clotrimazole treatment.

\section{Outcome measures}

All studies, with the exception of ACTRN12614000946617, Kumar 2001 and NCT01080079, addressed one or both primary objectives, reporting complete cure rates or adverse events, or both. Eight studies did not address mycological cure rate (EUCTR2005-005905-51; EUCTR2006-000974-54; NCT01080079; NCT01400594; NCT02022215; Romero-Cerecero 2009; Stein 2014; Syed 1998). We accepted mycological cure as reported by study investigators.

Twenty-eight studies defined mycological cure as negative $\mathrm{KOH}$ microscopy or PAS and negative culture (ACTRN12614001238662; Alberdi 2019; Auvinen 2015; Eertmans 2018; Elewski 2013a; Elewski 2013b; Elewski 2013c; Elewski 2013d; Elewski 2015a; Elewski 2015b; EUCTR2008-003215-13; Gupta 2000a; Gupta 2000b; Gupta 2006; Ijzerman 2010; Iorizzo 2016; Karsai 2017; Kumar 2001; Lahfa 2013; Lu 2005; NCT01246518; NCT02343627; NCT02933879; NijenhuisRosien 2019; Parekh 2017; Paul 2013; Syed 1999; Tschen 2013).

Studies also used less rigorous criteria, with three studies defining mycological cure as negative $\mathrm{KOH}$ microscopy alone (Amichai 2010; Romero-Cerecero 2008; Watanabe 2017), 10 studies as negative culture alone (ACTRN12614000946617; Baran 2009; Buck 1994; Hollmig 2014; Koren 2018; NCT03289871; Schalka 2012; Sigurgeirsson 2016; Toledo-Bahena 2014; Waibel 2013), three studies reported both microscopy and culture but separately (Bonhert 2019; Hartmane 2013; Montana 1994), two studies used molecular biology techniques (EUCTR2016-001242-25; NCT03141840), and two studies left cure undefined (EUCTR2006-005895-42; NCT01145807). Seven studies evaluated recurrence (Gupta 2000a; Gupta 2000b; Gupta 2006; Nijenhuis-Rosien 2019; Syed 1998; Syed 1999; Watanabe 2017), three studies provided pharmaco-economic information (Auvinen 2015; Buck 1994; Paul 2013), and two studies reported compliance with treatment (Auvinen 2015; Romero-Cerecero 2008).

No studies reported the impact of treatment on quality of life, but three studies stated that they would measure patientreported outcomes; one (Nijenhuis-Rosien 2019) did not include the outcome in the publication and two have yet to post results (EUCTR2016-001242-25; NCT03141840).

The most common assessment time point was 36 to 52 weeks from baseline, which was either immediately after treatment completion or within four-week follow-up after treatment completion. For some single studies 12 weeks and six months were also used as time point assessments from baseline, coinciding with treatment completion.

\section{Excluded studies}

We excluded 51 records from the review (see Characteristics of excluded studies). Of these, the most common reasons for exclusion were as follows:

- non-randomised studies (18 records: Anonymous 2000; Anonymous 2015; Bergstrom 2009; Botter 1971; Canavan 2018; Dina 2017; Fredriksson 1974; Furtado 2005; Hay 1988; Krasaeath 2016; Meyerson 1992; Morgado 2017; Rand 1996; Shemer 2016; Sotiriou 2010; Souza 2014; Tardivo 2015; Zaug 1995);

- combining the results of onychomycosis for toenails and fingernails, which prevented toenail data from being extracted (14 records: Bassiri-Jahromi 2012; El-Tatawy 2015; Emtestam 2012; Gilaberte 2017; Halmy 2005; Kim 2016b; Lauharanta 1992; Li 2014; Mahgoub 1975; Many 1964; Menéndez 2011; Tietz 2013; Zhang 2012a; Zhou 2016); 
- using nails as the unit of analysis rather than participants (six records: Kim 2016a; Landsman 2010; Landsman 2012; Lu 2016; Malay 2009; Reinel 1992);

- using a within-participant design as a control group (three records: Harris 2010; Ortiz 2014; Schaller 2017);

- less than 12 weeks in duration (three records: Chan 2005; Jimenez-Faraj 2018; Mensing 1992);

- using an ineligible treatment (three records: Schlockermann 1957 used a combination of surgery and antifungal medication; Gupta 2005 studied a combination of ciclopirox lacquer and oral terbinafine; Oberste-Lehn 1974 studied oral clotrimazole);

- Sigurgeirsson 2010 was an extension trial investigating amorolfine as prophylaxis in patients who achieved cure with oral treatment;

- Kamalam 1980 did not study onychomycosis, but rather fungal infections of the skin;

- NCT03094468 is a registered trial that has since been withdrawn due to project development priorities changing, and was excluded; and

- Abd El-Aal 2019 included both paediatric and adult participants.

\section{Studies awaiting classification}

Twenty-two records are classified as awaiting classification and are detailed in 'Characteristics of studies awaiting classification' (Anonymous 2002; Bagatell 1977; Barquero 2007; Butareva 1986; Emokpare 1977; EUCTR2008-002427-90; George 1991; Grant 1974; JPRN-JapicCTI-101143; Klima 1976; Kull 1972; Mathur 1973; NCT00253305; NCT02549001; Park 2017; Ramm 1994; Rüping 1993a; Rüping 1993b; Schubert 1973; Weuta 1972; Xu 2017; Yao 2016). We did not have enough information to assess these studies for inclusion, but they may be added to the review as information becomes available.

\section{Ongoing studies}

Thirteen records are ongoing studies from clinical trial registries and are detailed in 'Characteristics of ongoing studies' (ACTRN12618000131257; ChiCTR-INR-17013504; DRKS00007709; EUCTR2016-001204-39; EUCTR2016-003784-19;
JapicCTI-183840; NCT02644551; NCT02812043; NCT02859519; NCT02866032; NCT02961634; NCT03098342; NCT03814343). Any of these records may be added to the review as information becomes available.

Topical investigational products are being compared to vehicle or placebo, including HXP124 (ACTRN12618000131257), RJ-0265 (ciclopirox formulation) (EUCTR2016-003784-19), ME-1111 (JapicCTI-183840), CELEXT07 (NCT02644551), terbinafine HCl 10\% (NCT02859519), and amphotericin B (NCT03814343). Topical agents are also being compared to comparators: RJ-0265 versus ciclopirox 8\% (EUCTR2016-003784-19), ME-1111 versus efinaconazole (JapicCTI-183840), terbinafine $\mathrm{HCl} 10 \%$ solution versus ciclopirox 8\% (EUCTR2016-001204-39; NCT02866032), CELEXT07 versus ciclopirox 8\% (NCT02644551), and a combination of Nailner and ciclopirox 8\% versus ciclopirox 8\% (NCT02961634).

Photodynamic therapy is compared to amorolfine (NCT03098342). Three studies are investigating laser therapy: 1064-nm Nd:YAG laser (DRKS00007709) and 2940-nm Er:YAG (ChiCTR-INR-17013504), each in combination with amorolfine, versus amorolfine alone, and 1064-nm laser versus amorolfine versus combination laser plus amorolfine (NCT02812043).

\section{Risk of bias in included studies}

Two review authors ( $K F$ and $\mathrm{RM}$ ) independently assessed the included studies for risks of bias using the Cochrane 'Risk of bias' assessment tool as outlined in the Methods. We report assessments in the Characteristics of included studies, and summarise them in Figure 3 and Figure 4 . We judged three of the 56 studies to be at low risk across the seven domains (Elewski 2015a; Elewski 2015b; Nijenhuis-Rosien 2019). We rated seven studies at high risk in one domain but low risk for the other domains (Auvinen 2015; Eertmans 2018; Elewski 2013c; Elewski 2013d; Parekh 2017; Toledo-Bahena 2014; Tschen 2013), while 11 studies were at low risk on all but two domains (ACTRN12614000946617; Alberdi 2019; Buck 1994; EUCTR2016-001242-25; Hollmig 2014; Iorizzo 2016; Karsai 2017; Koren 2018; NCT02933879; NCT03141840; Sigurgeirsson 2016). Selective reporting (reporting bias) was the domain with the largest number of studies judged to be high risk. 
Figure 3. Risk of bias graph: review authors' judgements about each risk of bias item presented as percentages across all included studies.

Random sequence generation (selection bias)

Allocation concealment (selection bias)

Blinding of participants and personnel (performance bias)

Blinding of outcome assessment (detection bias)

Incomplete outcome data (attrition bias)

Selective reporting (reporting bias)

Other bias

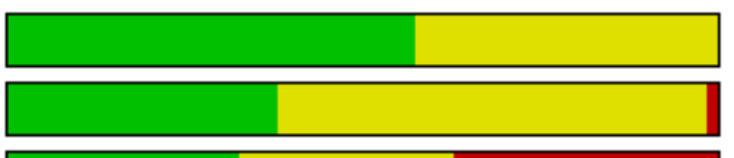

L

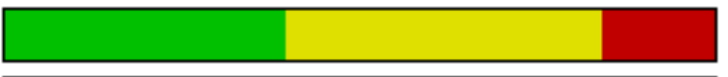

L
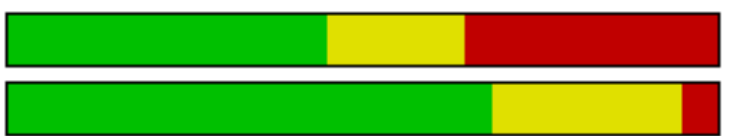

\begin{tabular}{|lllll}
\hline 1 & & 1 & 1 & \\
\hline $0 \%$ & $25 \%$ & $50 \%$ & $75 \%$ & $100 \%$
\end{tabular}

Low risk of bias

Unclear risk of bias

High risk of bias 
Figure 4. Risk of bias summary: review authors' judgements about each 'Risk of bias' item for each included study.

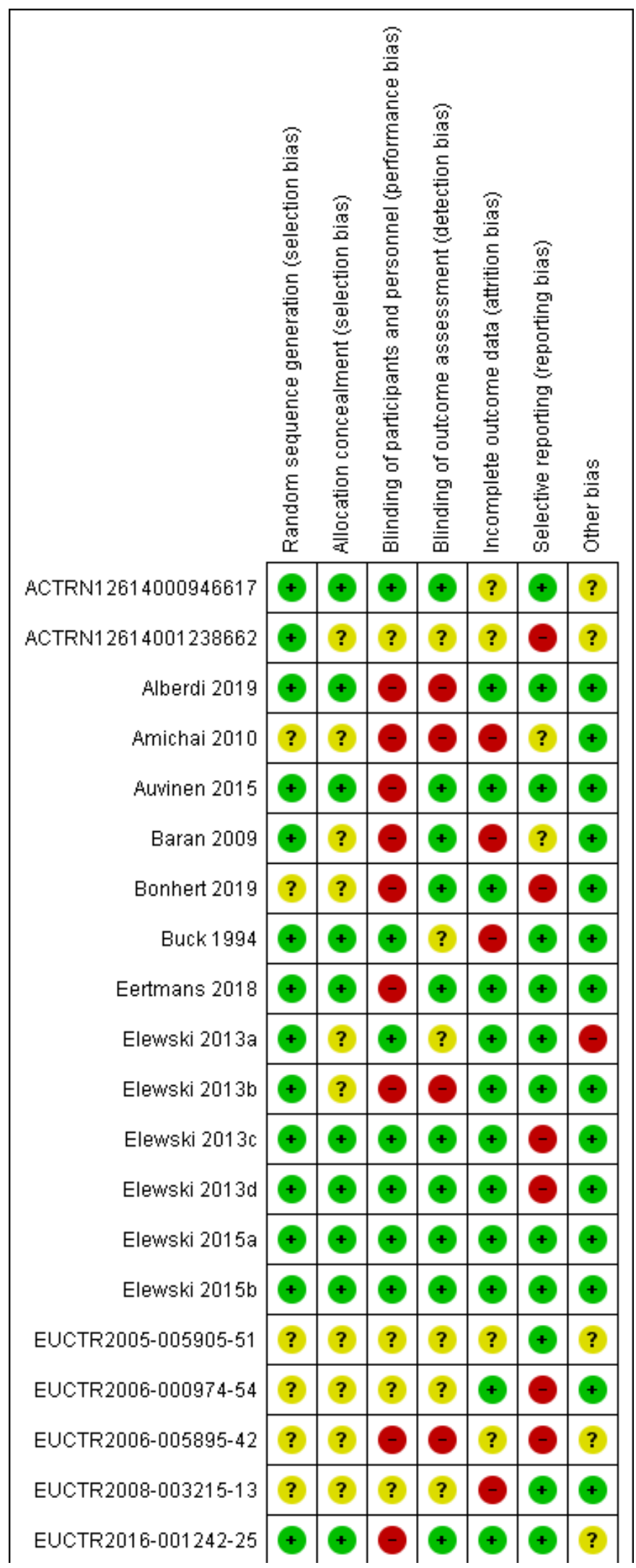


Figure 4. (Continued)

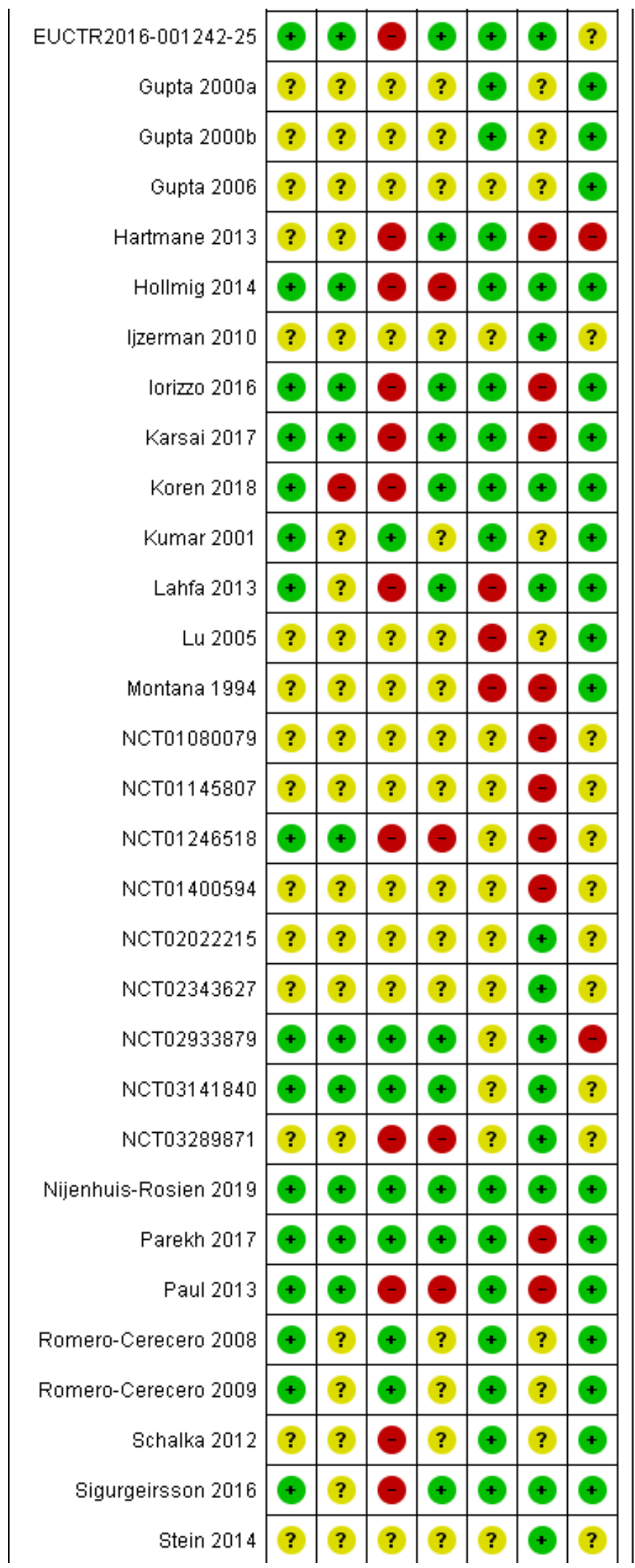


Figure 4. (Continued)

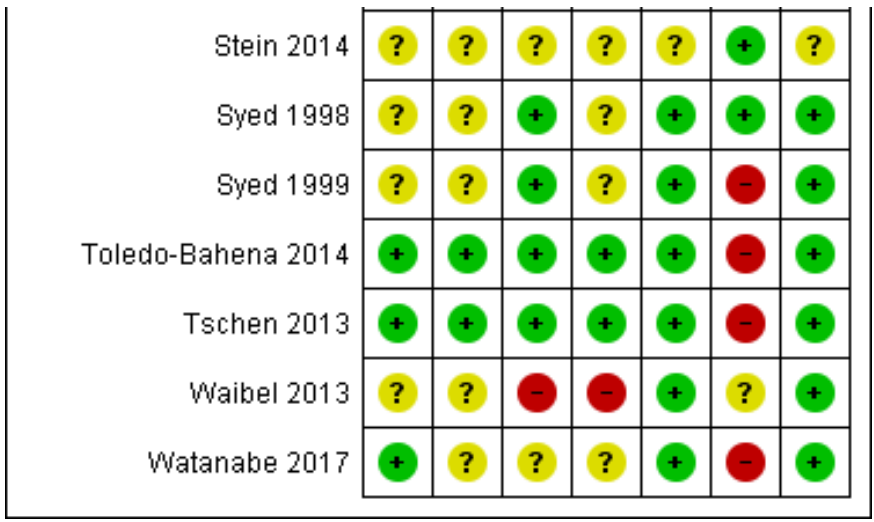

\section{Allocation}

\section{Sequence generation}

We judged 32 studies to be at low risk of bias for random sequence generation, as the sequence generation method was clearly stated in all of them (ACTRN12614000946617; ACTRN12614001238662; Alberdi 2019; Auvinen 2015; Baran 2009; Buck 1994; Eertmans 2018; Elewski 2013a; Elewski 2013b; Elewski 2013c; Elewski 2013d; Elewski 2015a; Elewski 2015b; EUCTR2016-001242-25; Hollmig 2014; Iorizzo 2016; Karsai 2017; Koren 2018; Kumar 2001; Lahfa 2013; NCT01246518; NCT02933879; NCT03141840; NijenhuisRosien 2019; Parekh 2017; Paul 2013; Romero-Cerecero 2008; Romero-Cerecero 2009; Sigurgeirsson 2016; Toledo-Bahena 2014; Tschen 2013; Watanabe 2017). We rated the remaining 24 studies at unclear risk of bias for sequence generation, as there was no information provided.

\section{Allocation concealment}

We judged 21 studies to be at low risk for selection bias due to clearly-stated allocation concealment procedures (ACTRN12614000946617; Alberdi 2019; Auvinen 2015; Buck 1994; Eertmans 2018; Elewski 2013c; Elewski 2013d; Elewski 2015a; Elewski 2015b; EUCTR2016-001242-25; Hollmig 2014; Iorizzo 2016; Karsai 2017; NCT01246518; NCT02933879; NCT03141840; Nijenhuis-Rosien 2019; Parekh 2017; Paul 2013; Toledo-Bahena 2014; Tschen 2013). We rated the remaining 35 studies as unclear due to a lack of information, except for Koren 2018, which we judged as high risk for selection bias, due to investigators being aware of randomisation procedures.

\section{Blinding}

\section{Performance bias}

We judged 18 studies to be at low risk for performance bias (ACTRN12614000946617; Buck 1994; Elewski 2013a; Elewski 2013c; Elewski 2013d; Elewski 2015a; Elewski 2015b; Kumar 2001; NCT02933879; NCT03141840; Nijenhuis-Rosien 2019; Parekh 2017; Romero-Cerecero 2008; Romero-Cerecero 2009; Syed 1998; Syed 1999; Toledo-Bahena 2014; Tschen 2013). These studies described procedures for ensuring that participants and study personnel were blinded. We rated 21 studies at high risk for performance bias, as these were unblinded or singleblinded studies (Alberdi 2019; Amichai 2010; Auvinen 2015; Baran 2009; Bonhert 2019; Eertmans 2018; Elewski 2013b; EUCTR2006-005895-42; EUCTR2016-001242-25; Hartmane 2013;
Hollmig 2014; Iorizzo 2016; Karsai 2017; Koren 2018; Lahfa 2013; NCT01246518; NCT03289871; Paul 2013; Schalka 2012; Sigurgeirsson 2016; Waibel 2013). The remaining 17 studies were at unclear risk, due to lack of information.

\section{Detection bias}

We judged 22 studies as being at low risk for detection bias, as it was clear in these studies that the individuals evaluating clinical outcomes were blinded to the treatment of participants (ACTRN12614000946617; Auvinen 2015; Baran 2009; Bonhert 2019; Eertmans 2018; Elewski 2013c; Elewski 2013d; Elewski 2015a; Elewski 2015b; EUCTR2016-001242-25; Hartmane 2013; Iorizzo 2016; Karsai 2017; Koren 2018; Lahfa 2013; NCT02933879; NCT03141840; Nijenhuis-Rosien 2019; Parekh 2017; Sigurgeirsson 2016; Toledo-Bahena 2014; Tschen 2013). We assessed nine studies as being at high risk as outcome assessment was not blinded (Alberdi 2019; Amichai 2010; Elewski 2013b; EUCTR2006-005895-42; Hollmig 2014; NCT01246518; NCT03289871; Paul 2013; Waibel 2013), with the remaining 25 studies judged as unclear due to a lack of information.

\section{Incomplete outcome data}

We judged 33 studies to be at low risk for attrition bias. These studies accounted for all participants and, where there were study withdrawals, reported study discontinuations and reasons for withdrawals for all study arms (Alberdi 2019; Auvinen 2015; Bonhert 2019; Eertmans 2018; Elewski 2013a; Elewski 2013b; Elewski 2013c; Elewski 2013d; Elewski 2015a; Elewski 2015b; EUCTR2006-000974-54; EUCTR2016-001242-25; Gupta 2000a; Gupta 2000b; Hartmane 2013; Hollmig 2014; Iorizzo 2016; Karsai 2017; Koren 2018; Kumar 2001; NijenhuisRosien 2019; Parekh 2017; Paul 2013; Romero-Cerecero 2008; Romero-Cerecero 2009; Schalka 2012; Sigurgeirsson 2016; Syed 1998; Syed 1999; Toledo-Bahena 2014; Tschen 2013; Waibel 2013; Watanabe 2017). We rated 16 studies at unclear risk as information was unavailable and not stated in the record (ACTRN12614000946617; ACTRN12614001238662; EUCTR2005-005905-51; EUCTR2006-005895-42; Gupta 2006; ljzerman 2010; NCT01080079; NCT01145807; NCT01246518; NCT01400594; NCT02022215; NCT02343627; NCT02933879; NCT03141840; NCT03289871; Stein 2014). We judged the remaining seven studies to be at high risk due to not stating reasons for participants discontinuing the study (Baran 2009; EUCTR2008-003215-13; Lahfa 2013; Lu 2005) or not specifying the 
treatment group to which participants who were lost to follow-up belonged (Amichai 2010; Buck 1994; Montana 1994).

\section{Selective reporting}

We judged 25 studies to be at low risk of reporting bias (ACTRN12614000946617; Alberdi 2019; Auvinen 2015; Buck 1994; Eertmans 2018; Elewski 2013a; Elewski 2013b; Elewski 2015a; Elewski 2015b; EUCTR2005-005905-51; EUCTR2008-003215-13; EUCTR2016-001242-25; Hollmig 2014; ljzerman 2010; Koren 2018; Lahfa 2013; NCT02022215; NCT02343627; NCT02933879; NCT03141840; NCT03289871; Nijenhuis-Rosien 2019; Sigurgeirsson 2016; Stein 2014; Syed 1998). The outcomes reported in these studies were similar to those listed in the studies' respective trial protocols, or where the trial protocol is the included record, clearly defined outcomes were stated, or, where a study pre-dates clinical trial registries, clearly defined outcomes were stated in the Methods section and reported in the Results. We rated 20 studies at high risk of bias where the outcomes were not clearly defined, or where there were discrepancies between outcomes stated in the trial registries and outcomes reported in publications (ACTRN12614001238662; Bonhert 2019; Elewski 2013c; Elewski 2013d; EUCTR2006-000974-54; EUCTR2006-005895-42; Hartmane 2013; Iorizzo 2016; Karsai 2017; Montana 1994; NCT01080079; NCT01145807; NCT01246518; NCT01400594; Parekh 2017; Paul 2013; Syed 1999; Toledo-Bahena 2014; Tschen 2013; Watanabe 2017). We judged the remaining 11 studies to be at unclear risk of bias, as we were unable to locate clinical trial registries to assess risks of bias (Amichai 2010; Baran 2009; Gupta 2000a; Gupta 2000b; Gupta 2006; Kumar 2001; Lu 2005; Romero-Cerecero 2008; RomeroCerecero 2009; Schalka 2012; Waibel 2013).

\section{Other potential sources of bias}

We judged 38 studies to be at low risk of other potential sources of bias. We determined this domain to be at high risk in three studies: Elewski 2013a pooled safety data for treatment groups, while Hartmane 2013 and NCT02933879 pooled clinical outcome data across treatment groups. Data per group were therefore not presented. We classified 15 studies as being at unclear risk of bias; these studies did not have results available to be able to assess other sources of bias (ACTRN12614000946617; ACTRN12614001238662; EUCTR2005-005905-51; $\quad$ EUCTR2006-005895-42; EUCTR2016-001242-25; ljzerman 2010; NCT01080079; NCT01145807; NCT01246518; NCT01400594; NCT02022215; NCT02343627; NCT03141840; NCT03289871; Stein 2014).

\section{Effects of interventions}

See: Summary of findings for the main comparison Ciclopirox $8 \%$ lacquer compared to vehicle for fungal infections of the toenails; Summary of findings 2 Efinaconazole $10 \%$ solution compared to vehicle for fungal infections of the toenails; Summary of findings 3 Tavaborole 5\% solution compared to vehicle for fungal infections of the toenails; Summary of findings 4 P-3051 (ciclopirox 8\% hydrolacquer) compared to comparators for fungal infections of the toenails; Summary of findings 5 1064-nm Nd:YAG laser compared to no treatment or sham treatment for fungal infections of the toenails; Summary of findings 6 Luliconazole 5\% solution compared to vehicle for fungal infections of the toenails

\section{Topical treatment versus vehicle/placebo}

\section{Ciclopirox $8 \%$ lacquer versus vehicle}

Two identical clinical trials (Gupta 2000a; Gupta 2000b) compared ciclopirox $8 \%$ lacquer with vehicle lacquer in a total of 460 participants (Summary of findings for the main comparison). Treatment duration was daily application for 48 weeks, with clinical outcomes also assessed at 48 weeks. There were no discernible differences in the trial populations. Population demographics of the two identical trials were similar, with predominantly male participants (study 1: $78.5 \%$, study 2: $77.2 \%$ ) and T. rubrum as the most common organism causing infection (study 1: $96.9 \%$, study 2: 95.4\%) (Gupta 2000a; Gupta 2000b).

\section{Primary outcomes}

\section{Complete cure}

See Analysis 1.1

Pooling these two clinical trials yielded 16 participants $(16 / 231=$ $6.9 \%$ ) achieving complete cure with ciclopirox $8 \%$ lacquer versus one participant $(1 / 229=0.4 \%)$ receiving vehicle lacquer (risk ratio (RR) $9.29,95 \%$ confidence interval (Cl) 1.72 to 50.14 ; $\mathrm{I}^{2}=0 \%$; NNTB = 3; low quality of evidence). Ciclopirox $8 \%$ lacquer may lead to complete cure for participants compared to those receiving vehicle lacquer.

\section{Adverse events}

See Analysis 1.2

There may be an increased risk of participants reporting adverse events deemed by investigators to be due to treatment with ciclopirox $8 \%$ lacquer $(26 / 231=11.3 \%)$ compared with vehicle lacquers $(16 / 229=7 \%)\left(\mathrm{RR} 1.61,95 \% \mathrm{Cl} 0.89\right.$ to $2.92 ; \mathrm{I}^{2}=0 \%$; low quality of evidence); however, the $95 \%$ indicates that ciclopirox lacquer may actually make little or no difference. The most commonly reported adverse events were application site reactions (transient tingling, burning, or pain with treatment use), rashes (mild erythema in the skin surrounding the nail), and alterations in nail colour or shape. These adverse reactions did not require additional treatment.

\section{Secondary outcomes}

\section{Mycological cure}

See Analysis 1.3

Thirty-one per cent of participants (71/231) achieved mycological cure, defined as negative $\mathrm{KOH}$ and negative culture, after treatment with ciclopirox $8 \%$ lacquer, while $9.6 \%$ of participants achieved mycological cure after treatment with vehicle lacquer (22/229). Mycological cure probably increases with ciclopirox $8 \%$ lacquer (RR $3.15,95 \% \mathrm{Cl} 1.93$ to $5.12 ; \mathrm{I}^{2}=16 \%$, NNTB $=2$; moderate quality of evidence).

\section{Clinical cure}

Clinical cure rate was not a clinical outcome in these studies.

\section{Compliance}

These studies did not report compliance. 


\section{Time to recurrence}

In these two trials (Gupta 2000a; Gupta 2000b), participants who achieved complete cure at 48 weeks with ciclopirox $8 \%$ lacquer or vehicle were followed for an additional 12 weeks. Of the 17 participants who were eligible, 10 were treatment failures. We were unable to perform analysis on these data as the treatment assignment of the participants followed was not available.

\section{Quality of life}

These studies did not include quality-of-life measures.

\section{Pharmaco-economics}

These studies did not include pharmaco-economic data.

\section{Efinaconazole $10 \%$ solution versus vehicle}

Two identical Phase 3 clinical trials (Elewski 2013c; Elewski 2013d) and a Phase 2 trial (Tschen 2013), compared efinaconazole 10\% solution with vehicle in a total of 1716 participants (Summary of findings 2). Treatment duration was daily application for 36 (Tschen 2013) or 48 weeks (Elewski 2013c; Elewski 2013d), with clinical outcomes assessed four weeks after the end of treatment. Tschen 2013 had multiple arms of efinaconazole and we selected for analysis the efinaconazole treatment arm consistent with Phase 3 trials and regulatory approval (10\% solution without semiocclusion). All arms of the Tschen 2013 trial are presented in Table 3.

\section{Primary outcomes}

\section{Complete cure}

\section{See Analysis 2.1}

Pooling these studies resulted in 215 participants $(215 / 1278=$ $16.8 \%$ ) receiving efinaconazole $10 \%$ solution achieving complete cure and 20 participants $(20 / 438=4.6 \%)$ receiving vehicle achieving complete cure (RR 3.54, 95\% Cl 2.24 to 5.60; $\mathrm{I}^{2}=3 \%$; NNTB = 2; high quality of evidence). Efinaconazole $10 \%$ solution leads to complete cure when compared to vehicle.

\section{Adverse events}

\section{See Analysis 2.2}

Participants were more likely to experience adverse events with efinaconazole $10 \%$ solution compared to vehicle (RR 1.10, 95\% Cl 1.01 to $1.20 ; 1^{2}=0 \%$, NNTH = 15; high quality of evidence). Sixty-six per cent of participants experienced an adverse event with efinaconazole $10 \%$ solution $(831 / 1266=65.6 \%)$ and $60 \%$ of participants experienced an adverse event with vehicle (261/435 $=60 \%)$. The most common adverse events reported in the Phase 3 trials (Elewski 2013c; Elewski 2013d) that were likely to be related to treatment were dermatitis (efinaconazole: 23/653 versus vehicle: $0 / 213$, Elewski 2013c) and vesicles (efinaconazole: 21/1227 versus vehicle 0/413, Elewski 2013c; Elewski 2013d) at the application site; most adverse events were probably not related to treatment application and included the common cold or nasopharyngitis (efinaconazole: 141/1227 versus vehicle 40/413), sinusitis (efinaconazole: 47/1227 versus vehicle: 9/413), and upper respiratory tract infection (efinaconazole: $73 / 1227$ versus vehicle: 24/413, Elewski 2013c; Elewski 2013d for all). Four adverse events related to efinaconazole were reported in the Phase 2 trial: one instance of ingrowing toenail in the efinaconazole $10 \%$ without semi-occlusion group (included in analysis) and one instance each of blister, contact dermatitis, and erythema in the efinaconazole $5 \%$ solution group (Tschen 2013).

\section{Secondary outcomes}

\section{Mycological cure}

See Analysis 2.3

Fifty-five per cent of participants $(706 / 1278=55.2 \%)$ achieved mycological cure, defined as negative $\mathrm{KOH}$ and negative culture, after treatment with efinaconazole $10 \%$ solution, while $19.6 \%$ of participants (86/438) achieved mycological cure after treatment with vehicle. Mycological cure probably increases with efinaconazole $10 \%$ solution ( $R R 2.31,95 \% \mathrm{Cl} 1.08$ to $4.94 ; \mathrm{I}^{2}=95 \%$; $\mathrm{NNTB}=2$; moderate quality of evidence). The large heterogeneity in the analysis could be due to the small sample size of Tschen 2013, or to the differences in treatment length and outcome assessment times between the Phase 2 (Tschen 2013) and Phase 3 (Elewski 2013c; Elewski 2013d) studies. In a sensitivity analysis removing Tschen 2013, results indicate that mycological cure increases with efinaconazole $10 \%$ solution (RR $3.22,95 \% \mathrm{Cl} 2.59$ to $4.01 ; \mathrm{I}^{2}=0 \%$, NNTB $=1$; high quality of evidence).

\section{Clinical cure}

See Analysis 2.4

Clinical cure was reported in Elewski 2013c and Elewski 2013d. Clinical cure increases with efinaconazole $10 \%$ solution $(242 / 1239$ $=19.5 \%)$ compared to vehicle $(26 / 416=6.3 \%)$; RR $3.07,95 \% \mathrm{Cl} 2.08$ to $4.53 ; 1^{2}=0 \%$, NNTB $=3$; high quality of evidence.

\section{Compliance}

These studies did not report compliance.

\section{Time to recurrence}

These studies did not assess time to recurrence.

\section{Quality of life}

These studies did not include quality-of-life measures.

\section{Pharmaco-economics}

These studies did not include pharmaco-economic data.

\section{Tavaborole $5 \%$ solution versus vehicle}

Two identical Phase 3 trials (Elewski 2015a; Elewski 2015b) compared tavaborole $5 \%$ solution versus vehicle in a total of 1198 participants (Summary of findings 3 ). Treatment duration was daily application for 48 weeks, with clinical outcomes assessed at 52 weeks.

\section{Primary outcomes}

\section{Complete cure}

See Analysis 3.1

Combining these two clinical trials yielded 62 participants $(62 / 799$ $=7.8 \%$ ) achieving complete cure with tavaborole $5 \%$ solution versus four participants $(4 / 399=1.0 \%)$ receiving vehicle (RR 7.40, $95 \% \mathrm{Cl} 2.71$ to $20.24 ; \mathrm{I}^{2}=0 \%$, NNTB $=2$; moderate quality of evidence). Treatment with tavaborole $5 \%$ solution probably increases complete cure rates compared to vehicle. 


\section{Adverse events}

\section{See Analysis 3.2; Analysis 3.3}

There was little or no difference between tavaborole $5 \%$ solution $(482 / 791=60.9 \%)$ and vehicle $(244 / 395=61.8 \%)$ in the number of participants reporting adverse events (RR $0.98,95 \% \mathrm{Cl} 0.85$ to 1.13 ; $1^{2}=56 \%$; high quality of evidence). The moderate heterogeneity in the analysis may be due to a difference in the number of participants with adverse events in the vehicle groups across the two studies. Any adverse event, whether or not related to treatment, is included in this analysis. More specifically, Elewski 2015a and Elewski 2015b identified adverse events related to treatment and analysis showed that tavaborole 5\% solution is probably more likely to lead to participants experiencing treatment-related adverse events $(48 / 791=6.1 \%)$ compared to participants receiving vehicle $(6 / 395=1.5 \%$; RR 3.82, 95\% Cl 1.65 to $8.85 ; 1^{2}=0 \%$; NNTB $=6$; moderate quality of evidence). Most reported adverse events related to treatment were application site reactions, and included exfoliation, dermatitis, erythema, and pain.

\section{Secondary outcomes}

\section{Mycological cure}

See Analysis 3.4

One-third of participants $(266 / 799=33.3 \%)$ achieved mycological cure, defined as negative $\mathrm{KOH}$ and negative culture, after treatment with tavaborole $5 \%$ solution, while $9.8 \%$ of participants (39/399) achieved mycological cure after treatment with vehicle. Mycological cure increases with tavaborole 5\% solution (RR 3.40, $95 \% \mathrm{Cl} 2.34$ to $4.93 ;\left.\right|^{2}=26 \%$; NNTB = 1; high quality of evidence).

\section{Clinical cure}

Clinical cure rate was not a clinical outcome in these studies.

\section{Compliance}

These studies did not report compliance.

\section{Time to recurrence}

These studies did not assess time to recurrence.

\section{Quality of life}

These studies did not include quality-of-life measures.

\section{Pharmaco-economics}

These studies did not include pharmaco-economic data.

\section{Topical treatment versus topical treatment}

\section{P-3051 (ciclopirox 8\% hydrolacquer) versus comparators}

Two studies (Baran 2009; lorizzo 2016) reported clinical trials comparing P-3051 (ciclopirox 8\% hydrolacquer) with other treatments in a total of 490 participants (Summary of findings 4). Baran 2009 compared P-3051 to ciclopirox 8\% lacquer, with both treatments applied daily for 48 weeks and clinical outcomes assessed at 52 and 60 weeks; this study also compared P-3051 to placebo but placebo data were not included in the analysis (see Table 3). Iorizzo 2016 compared P-3051 to amorolfine 5\%, applied daily or twice-weekly for 48 weeks, respectively, with clinical outcomes also assessed at 48 weeks.

\section{Primary outcomes}

\section{Complete cure}

See Analysis 4.1

Thirty-one participants $(31 / 242=12.8 \%)$ achieved complete cure with daily use of P-3051 (ciclopirox $8 \%$ hydrolacquer), while 13 participants $(13 / 248=5.2 \%)$ achieved complete cure with comparator treatments. P-3051 (ciclopirox $8 \%$ hydrolacquer) will probably lead to complete cure compared to comparator treatments (RR 2.43, 95\% Cl 1.32 to $4.48 ; \mathrm{I}^{2}=0 \%$; NNTB = 6; moderate quality of evidence). A post hoc analysis of Baran 2009 analysed participants with less than $50 \%$ nail involvement, whereas the clinical trial enrolled participants with up to $60 \%$ nail involvement. At 60 weeks, 18 participants $(18 / 119=15.1 \%)$ achieved complete cure with P-3051, while seven participants $(7 / 129=5.8 \%)$ achieved complete cure with the comparator ciclopirox $8 \%$ lacquer (RR 2.79, $95 \% \mathrm{Cl} 1.21$ to 6.44 ).

\section{Adverse events}

See Analysis 4.2

The risk of experiencing adverse events was similar for participants who received treatment with P-3051 (ciclopirox $8 \%$ hydrolacquer, $15 / 240=6.3 \%)$ or one of the comparators $(30 / 247=12.1 \%$; RR $0.60,95 \% \mathrm{Cl} 0.19$ to $1.92 ; \mathrm{I}^{2}=18 \%$; low quality of evidence). There were no adverse events related directly to P-3051 (Baran 2009; Iorizzo 2016) or to amorolfine 5\% (Iorizzo 2016), but two participants reported adverse events directly related to ciclopirox $8 \%$ lacquer (Baran 2009). The most commonly-reported adverse events reported by Baran 2009 included erythema, redness, and burning, while the one participant reporting an adverse reaction in lorizzo 2016 was unrelated to treatment (foot trauma). There may be little or no difference in adverse events experienced with ciclopirox $8 \%$ hydrolacquer and comparators (amorolfine $5 \%$ and ciclopirox $8 \%$ lacquer).

\section{Secondary outcomes \\ Mycological cure \\ See Analysis 4.3; Analysis 4.4}

The difference in mycological cure rates between P-3051 (ciclopirox $8 \%$ hydrolacquer) and comparator treatments indicates that there is probably little or no difference between the treatments (RR 1.08, $95 \% \mathrm{Cl} 0.85$ to $1.37 ; 1^{2}=91 \%$; NNTB = 13 ; moderate quality of evidence). Eighty-nine per cent of participants who received P-3051 experienced mycological cure $(216 / 242=89.3 \%)$, while $87.5 \%$ $(217 / 248)$ of participants who received comparator treatments experienced mycological cure. We did not perform sensitivity analysis on this outcome, as there were only two studies in the analysis. We conducted a sensitivity analysis to investigate the measure of effect in the population of participants who only had dermatophyte infections, as lorizzo 2016 included participants with dermatophyte, non-dermatophyte mould, or yeast infections, and Baran 2009 included participants with only dermatophyte infections. As in the full analysis, there is probably little or no difference between the treatments (RR $1.07,95 \% \mathrm{Cl} 0.85$ to 1.33 ; $\left.\right|^{2}=$ $88 \%$; moderate quality of evidence). An alternative explanation for the high heterogeneity may be differences in outcome definition. Baran 2009 defined mycological cure as negative culture, while lorizzo 2016 defined mycological cure as negative $\mathrm{KOH}$ and negative 
culture. As with complete cure, a post hoc analysis of Baran 2009 limited participants to less than $50 \%$ nail involvement. At 60 weeks, 98 participants $(98 / 119=82.4 \%)$ achieved negative culture, while 97 participants $(97 / 129=75 \%)$ achieved negative culture with the comparator ciclopirox $8 \%$ lacquer (RR 1.10, 95\% Cl 0.96 to 1.25).

\section{Clinical cure}

Clinical cure rate was not a clinical outcome in these studies.

\section{Compliance}

These studies did not report compliance.

\section{Time to recurrence}

These studies did not assess time to recurrence.

\section{Quality of life}

These studies did not include quality-of-life measures.

\section{Pharmaco-economics}

These studies did not include pharmaco-economic data.

\section{Device versus control}

\section{4-nm Nd:YAG laser versus no treatment/sham device}

Three studies (Hollmig 2014; Karsai 2017; Nijenhuis-Rosien 2019) reported clinical trials comparing 1064-nm Nd:YAG laser with no treatment or sham treatment in a total of 112 participants (Summary of findings 5). Two sessions (Hollmig 2014) or four sessions (Karsai 2017; Nijenhuis-Rosien 2019) of treatment with 1064-nm Nd:YAG were compared to no treatment or sham treatment, with clinical outcomes assessed at 12 (Hollmig 2014) and 52 weeks (Hollmig 2014; Karsai 2017; Nijenhuis-Rosien 2019).

\section{Primary outcomes}

\section{Complete cure}

Complete cure rate was not a clinical outcome in these studies.

\section{Adverse events}

See Analysis 5.1

There was no difference in adverse events (RR 4.85, 95\% Cl 0.24 to 97.11 ; Fishers exact test $P=0.24$; very low quality of evidence) between laser and sham treatment (Nijenhuis-Rosien 2019), with Karsai 2017 reporting no adverse events. Due to the very low quality of the evidence, we are uncertain about the adverse events that laser treatment may or may not deliver. During treatment, participants rating pain on a seven-point visual analogue scale (minimum 1, maximum 7) reported a median score of 5 with treatment (Karsai 2017).

\section{Secondary outcomes}

\section{Mycological cure}

See Analysis 5.2; Analysis 5.3

Due to the very low quality of the evidence, we are uncertain about the effects laser treatment may or may not have at 12 weeks. There was no difference in mycological cure, defined as negative culture (RR 1.18, $95 \% \mathrm{Cl} 0.26$ to 5.31; NNTB $=24$; Fishers exact test $P=1.0$; very low quality of evidence), between laser treatment and no treatment (Hollmig 2014). Twelve months after the end of treatment, there may be little to no difference in mycological cure (RR $1.04,95 \% \mathrm{Cl} 0.59$ to 1.85 ; NNTB = 80; low quality of evidence) between laser treatment and controls (Karsai 2017; NijenhuisRosien 2019).

Clinical cure

Clinical cure rate was not a clinical outcome in these studies.

\section{Compliance}

These studies did not report compliance.

\section{Time to recurrence}

These studies did not assess time to recurrence. Nijenhuis-Rosien 2019 defined recurrence as the same-species infection occurring at baseline and week 52, with negative biological results at week 30 . Three participants in the sham treatment group and six participants in the laser group experienced recurrent infection.

\section{Quality of life}

These studies did not include quality-of-life measures.

\section{Pharmaco-economics}

These studies did not include pharmaco-economic data.

\section{Description of studies not included in meta-analyses}

A number of studies met the inclusion criteria for this review, but these were single studies of interventions that we were unable to use in our meta-analyses. Whenever possible, we have included forest plots of studies published in the last four years (2014 to present) to illustrate the results of studies that may be considered emerging treatments, and to summarise the interventions and outcomes of the remaining studies. 'Summary of findings' tables are provided for interventions that may currently be clinically relevant.

\section{Topical treatment versus vehicle}

\section{Luliconazole $5 \%$ solution versus vehicle}

See Analysis 6.1; Analysis 6.2; Analysis 6.3; Summary of findings 6

Watanabe 2017 conducted a study comparing luliconazole 5\% solution with vehicle solution after 48 weeks of daily application. We are uncertain whether treatment with luliconazole $5 \%$ solution leads to complete cure, because we consider the evidence to be of very low quality (RR $2.96,95 \% \mathrm{Cl} 1.18$ to $7.41 ; 293$ participants; NNTB $=3$ ). There may be little or no difference in the occurrence of adverse events (RR 1.02, 95\% Cl 0.90 to 1.16; 293 participants; low quality of evidence) and mycological cure rate (negative $\mathrm{KOH}$ microscopy) (RR 1.39, 95\% Cl 0.98 to $1.97 ; 293$ participants; NNTB $=6$; low quality of evidence) between luliconazole $5 \%$ solution and vehicle. Commonly-reported adverse events were dry skin, contact dermatitis, paronychia, eczema, and hyperkeratosis; these improved or resolved immediately after stopping treatment. Participants who achieved complete cure with luliconazole $5 \%$ solution $(n=29)$ or vehicle $(n=5)$ were followed for an additional four weeks, with no participants experiencing recurrence during this time.

\section{Spirulina bioactive extract versus placebo}

See Analysis 7.1 
A bioactive extract, derived from Arthospira maxima (spirulina) which is a cyanobacterium used as food and marketed as AMYCOT, was compared to placebo (Parekh 2017). Each treatment was applied for a total of 24 weeks; for the first 12 weeks, lotion was applied twice daily and for the last 12 weeks once daily. Mycological cure (negative $\mathrm{KOH}$ microscopy and culture) did not differ between the two groups at six months (RR 2.20,95\% Cl 0.83 to 5.84; Fishers exact test $\mathrm{P}=0.17 ; 10$ participants). No participants reported adverse events in the bioactive extract arm. One participant in the placebo arm reported mild pain in both legs which was unrelated to treatment; as this study also included participants with skin infections, it was not clear if this participant had onychomycosis.

\section{Topical treatment versus topical treatment}

\section{Spruce resin lacquer versus amorolfine $5 \%$ lacquer}

See Analysis 8.1; Analysis 8.2; Analysis 8.3

A resin lacquer, $30 \%$ from the purified resin of the Norway spruce tree (Picea abies), was compared to amorolfine 5\% lacquer (Auvinen 2015). Each treatment was applied for nine months; resin lacquer $30 \%$ was applied once daily and amorolfine $5 \%$ lacquer was applied once weekly. Mycological cure (negative $\mathrm{KOH}$ microscopy and culture) was not different between the two groups at five months (RR $0.62,95 \% \mathrm{Cl} 0.21$ to $1.85 ; 48$ participants) or at 10 months (RR $1.63,95 \% \mathrm{Cl} 0.30$ to 8.90 ; Fishers exact test $\mathrm{P}=0.66$; 48 participants). No participants in either group achieved clinical cure at 12 to 36 or 36 to 52 weeks. No adverse events were reported for either treatment. There was no difference between treatment groups for self-reporting compliance, as compliance was close to $100 \%$ in both groups (RR $1.04,95 \% \mathrm{Cl} 0.93$ to 1.16 ; 48 participants). The cost of treatment per participant for nine months was reported to be EUR 41.60 for resin lacquer and EUR 56.30 for amorolfine.

\section{Amorolfine 5\% lacquer with nail varnish versus amorolfine $5 \%$ lacquer with no nail varnish}

See Analysis 9.1; Analysis 9.2

Sigurgeirsson 2016 compared treatment with amorolfine 5\% nail lacquer once weekly in the presence of nail varnish (applied 24 hours after treatment) versus no nail varnish once weekly for 12 weeks. After 12 weeks, there was no difference between groups in mycological cure, defined as negative culture (RR 1.00, 95\% Cl 0.90 to 1.12; 50 participants), and no clear difference in adverse events (RR 2.78, 95\% Cl 0.12 to 65.08; Fishers exact test $P=1.0 ; 50$ participants).

\section{Acetic acid-based peelable nail polish versus amorolfine 5\% lacquer}

See Analysis 10.1

An aqueous, acetic acid-based, peelable nail polish applied once daily for 180 days was compared to amorolfine $5 \%$ lacquer applied weekly for 180 days (Eertmans 2018). After 180 days, there was no difference between groups in mycological cure, defined as negative $\mathrm{KOH}$ (RR $1.11,95 \% \mathrm{Cl} 0.70$ to $1.75 ; 102$ participants). Scores on the nail quality-of-life scale decreased by 27.9 units for the peelable nail polish and by 26.7 units for amorolfine $5 \%$ lacquer compared to baseline, and did not differ from each other.

\section{Device versus device}

\section{Photodynamic therapy (PDT) versus Intense pulsed light (IPL)}

See Analysis 11.1; Analysis 11.2

Alberdi 2019 compared photodynamic therapy (PDT) mediated by methylene blue (MB) with intense pulsed light (IPL) treatment. All participants were pre-treated with $40 \%$ urea to soften nails followed by up to eight sessions of PDT or IPL spaced every one to two weeks. At week 28, there was no difference between groups in complete cure (RR $0.88,95 \% \mathrm{Cl} 0.57$ to $1.34 ; 46$ participants) or mycological cure, defined as negative PAS and negative culture (RR $0.88,95 \% \mathrm{Cl} 0.57$ to $1.34 ; 46$ participants).

\section{Combination therapy versus combination therapy}

Fractional ablative $\mathrm{CO} 2$ laser (FACL) + photodynamic therapy (PDT) versus FACL + amorolfine 5\% lacquer

See Analysis 12.1; Analysis 12.2

Fractional ablative $\mathrm{CO} 2$ laser (FACL) was applied to toenails prior to treatment with photodynamic therapy (PDT) mediated by 20\%-ALA or amorolfine 5\% lacquer (Koren 2018). At 12 and 36 weeks, there was no difference between groups in mycological cure, defined as negative culture (12 weeks: RR $1.31,95 \% \mathrm{Cl} 0.78$ to $2.19 ; 60$ participants; 36 weeks: RR 2.00, 95\% Cl 0.67 to 5.94 ; 60 participants).

\section{Other single studies}

There are 21 studies with reported data to describe (Table 3). Eight studies reported the primary outcome of complete cure (Elewski 2013a; Elewski 2013b; Lahfa 2013; NCT02933879; Romero-Cerecero 2008; Romero-Cerecero 2009; Syed 1998; Syed 1999) and 19 studies reported the primary outcome of adverse events (Amichai 2010; Buck 1994; Elewski 2013a; Elewski 2013b; EUCTR2006-000974-54; EUCTR2008-003215-13; Gupta 2006; Lahfa 2013; Montana 1994; NCT02343627; NCT02933879; Paul 2013; Romero-Cerecero 2008; Romero-Cerecero 2009; Schalka 2012; Syed 1998; Syed 1999; Toledo-Bahena 2014; Waibel 2013). Six studies reported the secondary outcome of clinical cure (Elewski 2013a; Elewski 2013b; Lahfa 2013; NCT02933879; Romero-Cerecero 2008; Romero-Cerecero 2009) while 17 studies reported mycological cure (Amichai 2010; Buck 1994; Elewski 2013a; Elewski 2013b; EUCTR2008-003215-13; Gupta 2006; Hartmane 2013; Kumar 2001; Lahfa 2013; Montana 1994; NCT02343627; NCT02933879; Paul 2013; Romero-Cerecero 2008; Schalka 2012; Toledo-Bahena 2014; Waibel 2013).

The outcomes reported most frequently were adverse events and mycological cure. Adverse events reported were mild to moderate, and resolved with cessation of treatment. Studies most commonly reported application-site reactions with treatment application, including local irritation/discomfort, erythema (redness), oedema or mild inflammation (swelling), pain or warmth, contact dermatitis, and tinea pedis (Amichai 2010; Buck 1994; Elewski 2013a; Elewski 2013b; EUCTR2006-000974-54; EUCTR2008-003215-13; Gupta 2006; Lahfa 2013; Montana 1994; NCT02343627; NCT02933879; Paul 2013; Romero-Cerecero 2008; Syed 1998; Syed 1999; Toledo-Bahena 2014). There were no adverse events reported with treatment of A.pichinchensis extract (RomeroCerecero 2008; Romero-Cerecero 2009) or ciclopirox 8\% lacquer (Schalka 2012), although other studies using ciclopirox $8 \%$ lacquer did report application-site reactions (Gupta 2000a; Gupta 2000b; 
Romero-Cerecero 2008). Acute pain was associated with laser use in Waibel 2013, which subsided once treatment was complete.

For mycological cure, studies reported statistical differences between treatment and vehicle or comparator for terbinafine nail solution (Elewski 2013a), and terbinafine 1\% patch with iontophoresis (Amichai 2010).

Mycological cure did not differ between active treatment and comparator for:

- 1064-nm laser (Waibel 2013);

- A. pichinchensis extract versus ciclopirox (Romero-Cerecero 2008);

- ciclopirox 8\% lacquer regimens (Schalka 2012);

- tea tree oil versus clotrimazole (Buck 1994);

- tavaborole $2.5 \%, 5 \%$, or $7.5 \%$ versus vehicle (Toledo-Bahena 2014);

- terbinafine nail solution versus amorolfine 5\% (Elewski 2013b);

- bifonazole versus placebo (both following urea $40 \%$ paste; EUCTR2008-003215-13);

- $40 \%$ urea ointment with plastic dressing versus bifonazole-urea combination (Lahfa 2013); and

- sequential treatment (40\% urea ointment with plastic dressing, ciclopiroxolamine cream, ciclopirox film-forming solution) versus amorolfine 5\% (Paul 2013).

No statistical analysis of mycological cure rates was provided in Gupta 2006, Hartmane 2013, Kumar 2001, Montana 1994, NCT02343627, or NCT02933879. Of the studies reporting mycological cure, Elewski 2013a, Elewski 2013b, Lahfa 2013, NCT02933879 and Romero-Cerecero 2008 reported our primary outcome of complete cure. There were no statistical differences between treatments in complete cure rates in any of these studies.

Lastly, there were 16 studies that met our inclusion criteria that did not have published results or did not have extractable data. Four of these studies were published conference abstracts or published studies, investigating a novel compound, NB-002 (ljzerman 2010), varying concentrations of terbinafine $\mathrm{HCl}$ lacquer ( $\mathrm{Lu}$ 2005), luliconazole $10 \%$ solution (Stein 2014), and efinaconazole $10 \%$ solution in combination with 1064-nm Nd:YAG laser (Bonhert 2019). There was not enough information to extract outcomes from these studies.

The remaining 12 studies are clinical trial registry entries (ACTRN12614000946617; ACTRN12614001238662; EUCTR2005-005905-51; $\quad$ EUCTR2006-005895-42; EUCTR2016-001242-25; NCT01080079; NCT01145807; NCT01246518; NCT01400594; NCT02022215; NCT03141840; NCT03289871) that have been completed; however, results are not posted or published. Four of these clinical trial registry entries are for trials registered in the year 2010 or earlier, with no updates past the year 2012 (EUCTR2005-005905-51; EUCTR2006-005895-42; NCT01080079; NCT01145807). It is possible that the results of these trials, as well as the conference abstracts which are also old, are negative and will not be published. The remaining eight trial registry entries were recently completed and data may be available at a later date (ACTRN12614000946617; ACTRN12614001238662; EUCTR2016-001242-25; NCT01246518; NCT01400594; NCT02022215; NCT03141840; NCT03289871).

\section{DISCUSSION}

\section{Summary of main results}

We included 56 studies (12,501 participants) in this review, with some publications reporting more than one study (e.g. Elewski 2013a; Elewski 2013b). We included 12 studies published in the year 2000 or later in our meta-analyses. The remaining 44 studies were single studies investigating various topical and device-based interventions that prevented further quantitative analysis, or they were clinical trial entries where the studies have been completed but results have not yet been posted. Eighty per cent of the studies assessed topical treatments alone, $9 \%$ device treatments, and $11 \%$ of the studies investigated both topical and device treatments, but there was a lack of included studies investigating amorolfine lacquer as an intervention of interest and urea creams/paste alone and not in combination treatments. The most common device included was the 1064-nm Nd:YAG laser. We were unable to include many device-based studies and even in the case of the 1064$\mathrm{nm} \mathrm{Nd}$ :YAG laser, there were only three included studies of laser monotherapy. Uncertainty in these areas therefore remains, as we cannot comment on the effectiveness of these interventions.

Clinical cure was measured for only one of our key comparisons and quality of life was not reported by any included study.

\section{Ciclopirox 8\% lacquer versus vehicle}

\section{Summary of findings for the main comparison}

Two studies in 460 participants (analysed for each of the following outcomes) provided low-quality evidence indicating that when compared to vehicle, ciclopirox $8 \%$ lacquer may be better at producing complete cure and is probably more effective in producing mycological cure. Ciclopirox $8 \%$ lacquer may lead to an increase in the number of participants reporting adverse events related to treatment; however, the $95 \%$ confidence interval indicates that ciclopirox $8 \%$ lacquer may actually make little or no difference (low-quality evidence). Common adverse events included reactions to treatment application such as tingling or burning, and mild rash. Clinical cure was not measured in these studies. Outcomes were measured after 48 weeks of treatment.

\section{Efinaconazole $10 \%$ solution versus vehicle}

\section{Summary of findings 2}

Two phase 3 studies and one phase 2 study, with a total of 1716 participants, provided high-quality evidence that efinaconazole $10 \%$ solution is more effective in achieving complete cure (1716 participants, 3 studies) and clinical cure (1655 participants, 2 studies) compared to vehicle. Efinaconazole $10 \%$ solution also leads to slightly more adverse events directly related to treatment than vehicle (high-quality evidence) (1701 participants, 3 studies), with application site reactions the most commonly reported events (e.g. dermatitis and vesicles). Mycological cure probably increases with efinaconazole $10 \%$ solution (moderate-quality evidence) (1716 participants, 3 studies). Treatment duration lasted either 36 weeks (one study) or 48 weeks (two studies), with clinical outcomes assessed four weeks after the end of treatment.

\section{Tavaborole $\mathbf{5} \%$ solution versus vehicle}

Summary of findings 3 
Two studies of 1198 participants (analysed for each of the following outcomes) provided high-quality evidence that when compared with vehicle, tavaborole $5 \%$ solution improves mycological cure, and moderate-quality evidence that it probably increases complete cure. However, it probably leads to more adverse events directly related to treatment than vehicle, with the most common adverse events reported being application-site reactions such as dermatitis, erythema, exfoliation, and pain (1186 participants, moderatequality evidence). Clinical cure was not measured in these studies. Treatment duration was 48 weeks, with clinical outcomes assessed at 52 weeks.

\section{P-3051 (ciclopirox 8\% hydrolacquer) versus comparators}

\section{Summary of findings 4}

P-3051 (ciclopirox 8\% hydrolacquer) was compared to either ciclopirox $8 \%$ lacquer or amorolfine $5 \%$ lacquer in two studies evaluating a total of 490 participants. Moderate-quality evidence suggests that P-3051 probably increases complete cure compared to comparators, and there is probably little or no difference between the treatments in producing mycological cure.

We found no evidence of a difference in the number of adverse events reported with P-3051 versus comparators (low-quality evidence) (487 participants). The most common adverse events reported included erythema, burning, and redness. Clinical cure was not measured in these studies. Treatment duration was 48 weeks, with clinical outcomes assessed at 48 to 52 weeks.

\section{4-nm Nd:YAG laser versus no treatment or sham treatment}

\section{Summary of findings 5}

Three studies compared 1064-nm Nd:YAG laser to no treatment or sham treatment. Very low-quality evidence means we are uncertain if there is a difference between groups in the number of participants experiencing adverse events ( 85 participants). There may be little or no difference in the laser's effect on mycological cure (measured at 52 weeks; 85 participants, low-quality evidence). Complete cure and clinical cure were not measured in these trials.

\section{Luliconazole $5 \%$ solution versus vehicle}

\section{Summary of findings 6}

Luliconazole $5 \%$ solution was investigated in a single study of 293 participants analysed for each of the following outcomes. Because of very low-quality evidence, we are uncertain of the comparative effect luliconazole $5 \%$ solution has on complete cure. There may be little or no difference in adverse events between luliconazole $5 \%$ solution and vehicle (low-quality evidence). There may be increased mycological cure with luliconazole $5 \%$ solution, but the $95 \%$ confidence interval indicates that luliconazole $5 \%$ solution may make little or no difference (lowquality evidence). Commonly-reported adverse events were dry skin, contact dermatitis, paronychia, eczema, and hyperkeratosis; these improved or resolved immediately after stopping treatment. This study did not measure clinical cure. Treatment was applied for 48 weeks, with outcomes assessed at the end of treatment.

\section{Overall completeness and applicability of evidence}

Major topical interventions for toenail onychomycosis were evaluated with meta-analyses: ciclopirox 8\% lacquer, P-3051 (ciclopirox $8 \%$ hydrolacquer), efinaconazole $10 \%$ solution, and tavaborole $5 \%$ solution. Included studies for each of these interventions reported our primary outcomes of complete cure and adverse events, as well as the secondary outcome of mycological cure. The most common device therapy, 1064-nm Nd:YAG laser, was evaluated with meta-analysis for adverse events and mycological cure. Mycological cure, defined as negative $\mathrm{KOH}$ microscopy and negative culture by regulatory authorities, is an informative outcome, indicating that the fungus causing the infection has been eradicated. However, only 28 studies used this definition. We accepted mycological cure as reported by study investigators. Large clinical studies will use this definition of mycological cure; smaller clinical studies will use one technique (e.g. microscopy alone) rather than a combination. An improvement in clinical appearance of the nail without eradication of the fungus may contribute to infection recurrence.

Complete cure is a suitable primary outcome, defined as mycological cure in addition to clearance of all clinical symptoms. Onychomycosis is difficult to treat and it is worth noting that complete cure rates are still low. Not all patients can expect to achieve complete cure with these treatments. We are confident that the results of this review are applicable to the general population afflicted with mild-to-moderate toenail onychomycosis caused by dermatophytes. Only one study enrolled severe onychomycosis. Every study in the analyses enrolled some participants with dermatophyte onychomycosis. Applicability of the results to infections caused by non-dermatophyte moulds or yeasts is uncertain, as only one RCT in our meta-analyses identified nondermatophyte or yeast infections alone (Iorizzo 2016). Of all the included studies, there were nine that included yeasts and five that included non-dermatophyte moulds, either alone or in combination with dermatophytes. We were unable to evaluate the remaining secondary outcomes of clinical cure, with the exception of the comparison of efinaconazole versus vehicle (studies often use varying definitions, making comparisons difficult); compliance; time to recurrence; quality of life; and pharmaco-economics. Few studies reported any of these outcomes, and no studies reported quality of life.

The review has externality validity, as it was able to evaluate the primary outcomes for most of the commonly-used topical and device-based treatments; however, we were unable to evaluate all of the currently relevant topical and device-based treatments for toenail onychomycosis. For example, we could not evaluate the evidence for use of urea-based treatment alone and amorolfine lacquer. These are older treatments where RCTs were, to our knowledge, either not conducted or published. Amorolfine is used as a comparator in our included trials (ACTRN12614000946617; Auvinen 2015; Elewski 2013b; lorizzo 2016; Koren 2018) but was not evaluated as the drug of interest, whereas urea is used in combination with topical treatments (EUCTR2008-003215-13; Lahfa 2013; Paul 2013; Syed 1998). In the case of laser therapy, there are many published studies for onychomycosis; however, only four of these (Hollmig 2014; Karsai 2017; Nijenhuis-Rosien 2019; Waibel 2013) met the inclusion criteria for this review, and only two could be included in a meta-analysis due to widely varying methods and outcome measures. Furthermore, we included only two studies of photodynamic therapy (PDT) and no meta-analysis was possible. There is therefore uncertainty about the effectiveness of devicebased treatments that we could not resolve. Clinicians, researchers and patients are interested in the potential of device-based 
therapies, but many questions remain about the effectiveness of lasers and other devices. Other topical treatments that we did not meta-analyse are not commonly used and require further evidence. These include region-specific treatments such as spruce resin lacquer, and $A$. pichinchensis that may be viable alternatives to common treatments (e.g. amorolfine, ciclopirox) in the future. Our results therefore do not sufficiently address the objective of the review: to assess the clinical and mycological effects of topical and device-based therapies for onychomycosis.

Two limitations that prevented studies of both topical and devicebased treatments from being included in this review deserve mention: we did not include RCTs that evaluated interventions in both toenails and fingernails and that did not present data in a way that allowed extraction of data for toenails alone.

Unit of analysis is also an issue, particularly with device-based studies. We set out to include studies in the current review that designated one toenail as the nail that was evaluated and usually this was the great (big) toenail; this was the case for studies of topical treatments. We found three laser studies where the number of people achieving cure could be evaluated, although these studies used many toenails per person (if a person was cured, all nails were cured). This allowed for inclusion of laser devices in this review, but we should emphasise that analysing data in this way is not in line with how drug trials are performed, and that this impedes our ability to evaluate devices in the same way as drugs and our ability to compare modalities. Most studies that include many toenails per person in the analysis will report the number of nails achieving cure, rather than the number of people achieving cure. A per-nail analysis may result in cure rates higher or lower than a per-person analysis, as people differ in responsiveness to treatment.

It is worth noting that there were 16 studies that met the inclusion criteria for this review but did not have results available. These studies were conference abstracts or clinical trial entries where the studies have been completed. Eight of these studies were last updated within three years; it is possible that data are forthcoming and may be included in a future review. The others have not been updated for some time, and highlight the difficulty in assessing investigative treatments.

\section{Quality of the evidence}

The five major comparisons for which meta-analyses were performed are summarised in 'Summary of findings' tables (Summary of findings for the main comparison; Summary of findings 2; Summary of findings 3; Summary of findings 4; Summary of findings 5). We also include a 'Summary of findings' table for one additional comparison in which there were single studies available and hence no meta-analyses performed (Summary of findings 6). We did this to highlight potential clinically relevant therapies for onychomycosis published in recent years.

\section{Limitations in study design or execution}

Most studies included in our meta-analyses did not raise concerns about methodology, and questions that arose were often addressed through author correspondence. The exceptions were ciclopirox 8\% lacquer and 1064-nm Nd:YAG laser; additional information was not available and we downgraded the evidence by one level due to missing information (unclear risk of bias) and lack of blinding, respectively. We also downgraded the evidence by one level for the single study of luliconazole $5 \%$ solution, due to missing information.

\section{Inconsistency of results}

For most of the outcomes analysed, results across studies were consistent and did not cause concern. We downgraded evidence for mycological cure with P-3051 (ciclopirox $8 \%$ hydrolacquer) and efinaconazole $10 \%$ solution by one level due to inconsistency. In both cases, there was evidence of heterogeneity among studies. Adverse events of P-3051 and 1064-nm Nd:YAG laser were also downgraded by one level due to wide variation in the effects observed across studies. Wide variation in the effect observed for mycological cure at 12 weeks in a single study of laser therapy resulted in downgrading evidence by one level.

\section{Indirectness of results}

We did not downgrade any studies due to indirectness. Participants in studies were representative of the general patient population. Interventions assessed were similar to what would be observed in clinical practice and were applied by the participants. All studies reported outcomes at appropriate time points.

\section{Imprecision of results}

There were few studies in our analyses. Imprecision was due mostly to small sample sizes which lead to a small number of detected events and large confidence intervals. We downgraded evidence by one level for complete cure in the ciclopirox $8 \%$ lacquer and tavaborole $5 \%$ solution analyses, for adverse events in the P-3051 and tavaborole $5 \%$ solution analyses, for mycological cure in the P-3051 and 1064-nm Nd:YAG laser analysis, and by two levels for adverse events in the laser analysis. We also downgraded the single study of luliconazole by one level for adverse events and mycological cure, and by two levels for complete cure.

\section{Publication bias}

We did not downgrade evidence for publication bias. The comprehensive literature search is very likely to have limited bias for our comparisons. However, it is possible that older clinical trial registries and conference abstracts produced negative results and were not published. Even if results do not warrant publication in a peer-reviewed journal, it would be informative if negative results were posted to clinical trial registries.

\section{Potential biases in the review process}

An obvious potential source of bias is that the lead author of this review is also the lead author of one included study comparing ciclopirox 8\% lacquer to vehicle (Gupta 2000a; Gupta 2000b; Summary of findings for the main comparison) and an author on additional studies on terbinafine nail lacquer (Elewski 2013a; Elewski 2013b; Gupta 2006) and tavaborole (Elewski 2015a; Elewski 2015b). To prevent this from affecting the review process, other review authors (KF, SV, RM) who have academic research backgrounds and are not clinicians performed the screening and selection of sources to be included in the review, as well as the evaluation of risks of bias and judging evidence quality with GRADE. The lead author (AKG) accepted the judgements of the academic team and participated in all other facets of the review, including clinical and research implications. 
We made every attempt to be objective and avoid bias in evaluating the evidence. We conducted an extensive search for eligible studies, and contacted study authors for additional data and methodological details. However, the fact that 22 studies awaiting classification have not yet been incorporated may be a potential source of bias. Additionally, incomplete correspondence with study investigators and sponsors limited our ability to assess risks of bias, particularly of randomisation and allocation concealment. We were unable to obtain results or updates on results for many of the clinical trial registry entries.

Toenails and fingernails have different rates of growth, with fingernails growing faster than toenails. This means that fingernails may respond more favourably to interventions. As fingernail onychomycosis is not as common as toenail onychomycosis, including a few fingernails in a clinical study could inflate the cure rates and misrepresent the efficacy of the intervention. We excluded studies where data were reported for fingernails and toenails combined, and we might therefore have excluded some potentially useful data on toenails. Lastly, after seeing the data, we decided to include forest plots of single studies of treatments published since 2014. We considered that these may be emerging treatments that stakeholders should know about.

At least two authors independently screened and selected studies, extracted data, assessed risks of bias, and graded the quality of the evidence.

\section{Agreements and disagreements with other studies or reviews}

When the last Cochrane Review on topical treatments for fungal infections of the toenail was published (Crawford 2007), there was very limited information available and the review also included topical treatments for fungal infection of the skin of the foot. Most of the studies included in the present review were published since 2007, justifying the need for a comprehensive review of toenails alone. Consequently, the information in the present review does not agree or disagree with the previous review, and offers new information to the evidence base. The one exception to this is the use of ciclopirox $8 \%$ lacquer for fungal infections of the toenails. Crawford 2007 concluded that ciclopirox 8\% lacquer was a poor choice for nail infections as cure rates were low. This is similar to the conclusion of our review, where we have found lowquality evidence to suggest that ciclopirox $8 \%$ lacquer may lead to complete cure compared with vehicle.

In a network meta-analysis using RCTs of oral and topical onychomycosis treatments, the odds ratios of achieving mycological cure for efinaconazole $10 \%$ solution, tavaborole $5 \%$ solution, and ciclopirox $8 \%$ lacquer were each found to be significantly superior to vehicle (Gupta 2015b). This is consistent with the current review's results as moderate-quality (ciclopirox $8 \%$ lacquer, efinaconazole $10 \%$ solution) or high-quality evidence (tavaborole 5\% solution) supports these topical treatments producing mycological cure.

Odds ratios for terbinafine nail solution and amorolfine 5\% lacquer were also found to be significantly superior to vehicle (Gupta 2015b). Gupta 2015b included trials with a minimum 48 weeks duration and did not include Paul 2013; since this analysis was published, additional studies using amorolfine have been published (e.g. Auvinen 2015; Koren 2018; Sigurgeirsson
2016). Our review was unable to perform a meta-analysis for terbinafine nail solution and amorolfine, as studies with overlapping comparisons were lacking (ACTRN12614000946617; Auvinen 2015; Eertmans 2018; EUCTR2006-000974-54; Iorizzo 2016; Koren 2018; NCT03289871; Paul 2013; Sigurgeirsson 2016). This highlights the advantages of the network meta-analysis technique; although the evidence network may be thin, one can be created using a limited number of studies for a comparison. Repeating the analysis of Gupta 2015b while including all treatments would allow the amorolfine studies our review identified to be analysed in a similar network. To our knowledge, there has not been a network meta-analysis evaluating complete or clinical cure.

Based on low-quality evidence and very few studies, the results of our review suggest that there is uncertainty about the effectiveness of laser treatment for onychomycosis. A systematic review published in 2014 concluded that the evidence for laser treatment was limited and identified methodological concerns (Bristow 2014), which included small sample sizes, lack of control groups, and variable follow-up periods. Gupta 2017 reports weighted pooled rates for mycological cure, but the lack of control data and consistently-defined clinical outcomes precluded further quantitative analysis. We have included only four studies of laser therapy in our review, and the reasons for excluding other laser studies reflected the concerns of the aforementioned reviews, namely, many laser studies are not randomised, do not employ control groups, and/or report data by nail rather than by participant.

Currently, device-based treatments are not approved for treatment of onychomycosis in the USA (e.g. are not approved to produce complete or mycological cure) but rather, are approved to temporarily increase the amount of clear nail (Lipner 2019; U.S. FDA 2015).

In patients that achieve complete cure, recurrence is a possibility. Although we were unable to report time to recurrence in this review, recurrence is common in toenail onychomycosis. Rates of recurrence following oral antifungal treatment in the published literature range from 10\% to 53\% (Piraccini 2010; Sigurgeirsson 2002; Tosti 1998), with recurrence more likely to occur within the first two years following cure (Sigurgeirsson 2002). Recurrence following topical or device-based treatments should be considered a possibility.

\section{AUTHORS' CONCLUSIONS}

\section{Implications for practice}

Some topical treatments for toenail onychomycosis are a viable alternative to traditional oral therapies in mild-to-moderate severity of onychomycosis, although it should be noted that we did not compare topical therapies to oral therapies in this review.

In comparison with vehicle, there is high-quality evidence that efinaconazole $10 \%$ solution is more effective in achieving complete cure, low-quality evidence that ciclopirox $8 \%$ lacquer may better lead to complete cure, and moderate-quality evidence in support of tavaborole $5 \%$ solution and P-3051 (ciclopirox $8 \%$ hydrolacquer) probably being more likely to achieve complete cure, although for P-3051 the comparators are ciclopirox 8\% lacquer or amorolfine $5 \%$, rather than vehicle. However, not all patients can be expected 
to achieve complete cure, since reported cure rates in clinical studies, while better than vehicle, are still low.

Treatment-related adverse events with efinaconazole (slightly increases adverse events compared with vehicle; high-quality evidence) and tavaborole (probably increases adverse events compared with vehicle; moderate-quality evidence) were limited to application-site reactions, with erythema, rash, and burning reported for P-3051 and its comparators ciclopirox 8\% lacquer or amorolfine $5 \%$, although for this comparison adverse events may not have been directly related to treatment, and we found no evidence of a difference in the risk of adverse events between the groups (low-quality evidence). For ciclopirox $8 \%$ lacquer, application site reactions, rashes, and alterations in nail colour or shape were the most common events, and this intervention may lead to an increase in the number of participants reporting adverse events related to treatment compared with vehicle; however, the $95 \%$ confidence interval indicates that ciclopirox $8 \%$ lacquer may actually make little or no difference (low-quality evidence).

In terms of mycological cure, when compared with vehicle, tavaborole $5 \%$ solution increases this outcome, and ciclopirox $8 \%$ lacquer and efinaconazole $10 \%$ probably increase the outcome. For mycological cure, there is probably little or no difference between P-3051 (ciclopirox 8\% hydrolacquer) and its comparators (ciclopirox $8 \%$ lacquer or amorolfine 5\%).

Studies evaluating ciclopirox 8\%, P-3051, efinaconazole, or tavaborole topical treatments included infections caused by dermatophytes. Infections caused by non-dermatophyte moulds, yeast, or mixed infections (a combination of dermatophytes and nondermatophyte moulds or yeast) may also benefit from these treatments and their use in everyday practice will be dictated by regulatory restrictions, clinicians' experience, and patient preferences. The study populations were representative of the general population afflicted with mild-to-moderate toenail onychomycosis caused by dermatophytes.

There is currently not enough evidence to recommend or discourage the use of luliconazole $5 \%$ solution, 1064-nm Nd:YAG laser, or photodynamic therapy. We included a small number of device-based studies and could not meet our objective in drawing conclusions on the clinical and mycological effectiveness of devicebased interventions. There were a number of topical interventions about which we could not draw conclusions (Table 3). These include combinations of creams with urea, and treatments specific to geographic areas such as Finland (spruce resin lacquer) and Mexico (A. pichinchensis). We could not evaluate treatments that no published RCTs have assessed, such as amorolfine or urea-based treatment alone.

Only one of our key comparisons measured clinical cure: efinaconazole $10 \%$ is more effective in achieving this outcome when compared with vehicle; high-quality evidence. Most of the included trials did not measure the secondary outcomes of compliance, time to recurrence, or pharmaco-economics, with no studies reporting quality of life.

The 22 studies in Studies awaiting classification may alter the conclusions of the review once assessed.

\section{Implications for research}

More randomised controlled studies of device-based therapies (e.g. 1064-nm Nd:YAG, photodynamic therapy, iontophoresis) are needed. Unlike drug treatments, there are no generally accepted outcomes that device-based studies adopt. The US Food and Drug Administration (FDA) has published non-binding guidance suggesting objective clinical measures that could be used for onychomycosis treatment, including mycological cure, defined as negative microscopy and negative culture (U.S. FDA 2015). Additional clinical outcomes that have been suggested for devices differ from those used for drug treatments; rather than focusing on complete cure, the non-binding guidance suggests $0 \%$ nail involvement (clinical cure) if less than $12 \mathrm{~mm}$ of nail is infected prior to treatment (U.S. FDA 2015).

Patient-centred outcomes are lacking in clinical studies. Further research could be conducted into patient satisfaction with treatment results, improvements in quality of life (e.g. social interactions, confidence), and compliance (e.g. ease and convenience of the intervention). Informal discussion has taken place as to what realistic and useful clinical outcomes may be for onychomycosis studies (Elewski 2016). Complete cure rates for topical treatments, while better than vehicle, are low, and there are no other consistently-reported clinical outcomes. It may be worth considering attempts in line with Cochrane Skin Core Outcomes Set Initiative to guide studies worldwide (CS-COUSIN). For both drugand device-based studies, clinical outcomes should be reported at a patient level, i.e. one target nail for analysis, usually the great (big) toenail.

Research to characterise therapy efficacy in patients with severe onychomycosis is warranted, as well as those with mild-tomoderate onychomycosis. Data are absent on the recurrence of onychomycosis following topical and device-based treatment, and given documented recurrence with oral antifungal therapy, the same should be investigated with topical and device-based interventions.

Of concern to both patients and physicians is the cost of treatments for onychomycosis. Clinical studies very rarely undertake costeffectiveness analysis.

There is also a paucity of studies that perform head-to-head comparisons of topical treatments. For example, there are no direct comparisons of amorolfine and ciclopirox with efinaconazole, luliconazole, or tavaborole. Device-based treatments have not been compared to topical treatments in randomised controlled trials. Direct comparisons of treatments in clinical studies with the inclusion of cost-effectiveness data will provide valuable information that can aid clinicians and patients in making treatment decisions. Network meta-analysis may be considered in the future to overcome the lack of direct comparisons in clinical studies. Further research into, for example, region-specific treatments, such as spruce resin lacquer or A. pichinchensis, or urea-based treatments, would provide evidence for further onychomycosis treatment options.

Future investigations of topical and device-based interventions would benefit from characterising outcomes of success according to causative organism. Current studies focus on dermatophyte infections and evidence for treatment success in nondermatophyte and mixed infections would be informative. All future studies, both 
topical and device-based, should strive to maintain blinding of at least participants and outcome assessors. Adequate sample size will limit imprecision associated with small numbers of detected events. Heterogeneity among studies may be limited by characterising outcomes according to causative organism and, in the case of mycological cure, adopting the definition of negative $\mathrm{KOH}$ microscopy and negative culture.

Regardless of the modality, topical or device, future randomised controlled trials should adhere, and continue to adhere, to the reporting standards laid out in the Consolidated Standards of Reporting Trials (CONSORT) statement (Schulz 2010), to allow a fair and accurate assessment of the evidence for these interventions.

\section{ACK N O WLEDGEMENTS}

The authors wish to acknowledge Dr Jessie L Carviel for creation of Figure 1 and Melissa A MacLeod, MSc, for guidance about referees' comments. Thank you to Professor Erika Ota and Dr Christian Surber for help translating articles. Additionally, thank you to the many individuals who took the time to respond to our inquiries about their studies.

The Cochrane Skin Group editorial base wishes to thank Sue Jessop who was the Cochrane Dermatology Editor; Jeremy Hugh who was the External Content Expert; Laurence Le Cleach who was the Methods Editor for this review; Matthew Grainge who was the Statistical Editor; and the consumer referee, Muhammad Imran Omar. We would also like to thank Kate Cahill for copy-editing the review. 


\section{RE F E R E N C E S}

\section{References to studies included in this review \\ ACTRN12614000946617 \{unpublished data only\}}

ACTRN12614000946617. Kunzea oil for the management of fungal nail infection (toenail onychomycosis), a pilot randomised controlled trial. www.anzctr.org.au/Trial/ Registration/TrialReview.aspx?id=366923 (first received 4 September 2014). [CENTRAL: CN-01831590]

\section{ACTRN12614001238662 \{unpublished data only\}}

ACTRN12614001238662. Evaluation of mastic paint in the treatment of nail disorders (onycholysis, onychomycosis, onychorrhexis). www.anzctr.org.au/Trial/Registration/ TrialReview.aspx?ACTRN=12614001238662 (first received 26 November 2014).

\section{Alberdi 2019 \{published data only\}}

Alberdi E, Gomez C. Efficiency of methylene blue-mediated photodynamic therapy vs intense pulsed light in the treatment of onychomycosis in the toenails. Photodermatology, Photoimmunology, and Photomedicine 2019;35(2):69-77. [CENTRAL: CN-01951889]

\section{Amichai 2010 \{published data only\}}

Amichai B, Nitzan B, Mosckovitz R, Shemer A. Iontophoretic delivery of terbinafine in onychomycosis: a preliminary study. British Journal of Dermatology 2010;162(1):46-50. [CENTRAL: $\mathrm{CN}-00741789]$

\section{Auvinen 2015 \{published data only\}}

Auvinen T, Tiihonen R, Soini M, Wangel M, Sipponen A, Jokinen JJ. Efficacy of topical resin lacquer, amorolfine and oral terbinafine for treating toenail onychomycosis: a prospective, randomized, controlled, investigator-blinded, parallel-group clinical trial. British Journal of Dermatology 2015;173(4):940-8. [CENTRAL: CN-01105172]

\section{Baran 2009 \{published data only\}}

* Baran R, Tosti A, Hartmane I, Altmeyer P, Hercogova J, Koudelkova V, et al. An innovative water-soluble biopolymer improves efficacy of ciclopirox nail lacquer in the management of onychomycosis. Journal of the European Academy of Dermatology and Venereology 2009;23(7):773-81. [CENTRAL: CN-00718755]

Piraccini BM, Tosti A. Ciclopirox hydroxypropyl chitosan: efficacy in mild-to-moderate onychomycosis. Skin Appendage Disorders 2019;5(1):13-9. [CENTRAL: CN-01611757]

\section{Bonhert 2019 \{published data only\}}

Bonhert K, Dorizas A, Sadick NS. Efficacy of combination therapy with efinaconazole $10 \%$ solution and $1064 \mathrm{~nm} \mathrm{Nd:YAG}$ laser for treatment of toenail onychomycosis. Journal of Cosmetic and Laser Therapy 2019;21(3):179-83. [CENTRAL: CN-01629520]

\section{Buck 1994 \{published data only\}}

Buck DS, Nidorf DM, Addino JG. Comparison of two topical preparations for the treatment of onychomycosis: Melaleuca alternifolia (tea tree) oil and clotrimazole. Journal of Family Practice 1994;38(6):601-5. [CENTRAL: CN-00101608]

\section{Eertmans 2018 \{published data only\}}

Eertmans F, Doss N, Rossel B, Adriaens E. Daily application of an aqueous, acidifying, peelable nail polish versus weekly amorolfine for topical onychomycosis treatment: a prospective, randomized, blinded trial. Dermatology and Therapy 2018;8(3):463-73. [CENTRAL: CN-01646389]

\section{Elewski 2013a \{published data only\}}

Elewski BE, Ghannoum MA, Mayser P, Gupta AK, Korting HC, Shouey RJ, et al. Efficacy, safety and tolerability of topical terbinafine nail solution in patients with mild-to-moderate toenail onychomycosis: results from three randomized studies using double-blind vehicle-controlled and open-label activecontrolled designs. Journal of the European Academy of Dermatology and Venereology 2013;27(3):287-94. [PUBMED: 22181693]

\section{Elewski 2013b \{published data only\}}

Elewski BE, Ghannoum MA, Mayser P, Gupta AK, Korting HC, Shouey RJ, et al. Efficacy, safety and tolerability of topical terbinafine nail solution in patients with mild-to-moderate toenail onychomycosis: results from three randomized studies using double-blind vehicle-controlled and open-label activecontrolled designs. Journal of the European Academy of Dermatology and Venereology 2013;27(3):287-94. [PUBMED: 22181693]

\section{Elewski 2013c \{published and unpublished data\}}

* Elewski BE, Rich P, Pollak R, Pariser DM, Watanabe S, Senda H, et al. Efinaconazole $10 \%$ solution in the treatment of toenail onychomycosis: two phase III multicenter, randomized, doubleblind studies (Erratum appears in Journal of the American Academy of Dermatology, 2014 Feb;70(2):399). Journal of the American Academy of Dermatology 2013;68(4):600-8. [CENTRAL: CN-00966732]

Gupta AK, Elewski BE, Sugarman JL, leda C, Kawabata H, Kang R, et al. The efficacy and safety of efinaconazole $10 \%$ solution for treatment of mild to moderate onychomycosis: a pooled analysis of two phase 3 randomized trials. Journal of Drugs in Dermatology 2014;13(7):815-20. [CENTRAL: CN-01041119]

\section{Elewski 2013d \{published and unpublished data\}}

* Elewski BE, Rich P, Pollak R, Pariser D M, Watanabe S, Senda $\mathrm{H}$, et al. Efinaconazole $10 \%$ solution in the treatment of toenail onychomycosis: two phase III multicenter, randomized, double-blind studies (Erratum appears in Journal of the American Academy of Dermatolology, 2014 Feb;70(2):399). Journal of the American Academy of Dermatology 2013;68(4):600-8. [CENTRAL: CN-00966732]

Gupta AK, Elewski BE, Sugarman JL, leda C, Kawabata H, Kang R, et al. The efficacy and safety of efinaconazole $10 \%$ solution for treatment of mild to moderate onychomycosis: a pooled analysis of two phase 3 randomized trials. Journal 
of Drugs in Dermatology 2014;13(7):815-20. [CENTRAL: CN-01041119]

\section{Elewski 2015a \{published data only\}}

* Elewski BE, Aly R, Baldwin SL, Gonzalez Soto RF, Rich P, Weisfeld $M$, et al. Efficacy and safety of tavaborole topical solution, $5 \%$, a novel boron-based antifungal agent, for the treatment of toenail onychomycosis: Results from 2 randomized phase-III studies. Journal of the American Academy of Dermatology 2015;73(1):62-9. [CENTRAL: CN-01085211]

Gupta AK, Hall S, Zane LT, Lipner SR, Rich P. Evaluation of the efficacy and safety of tavaborole topical solution, $5 \%$, in the treatment of onychomycosis of the toenail in adults: a pooled analysis of an 8-week, post-study follow-up from two randomised phase 3 studies. Journal of Dermatological Treatment 2018;29(1):44-8. [CENTRAL: CN-01458612]

Pariser DM, Wendelken ME, Rycerz AM, Gellings Lowe N, Yost JM, Lipner SR. Planimetric post-hoc analysis of women with onychomycosis from tavaborole $5 \%$ phase III studies: evidence of greater improvements in patients with $>50 \%$ baseline infection. Journal of Drugs in Dermatology 2018;17(2):168-72. [CENTRAL: CN-01627312]

\section{Elewski 2015b \{published data only\}}

* Elewski BE, Aly R, Baldwin SL, Gonzalez Soto RF, Rich P, Weisfeld $M$, et al. Efficacy and safety of tavaborole topical solution, $5 \%$, a novel boron-based antifungal agent, for the treatment of toenail onychomycosis: Results from 2 randomized phase-III studies. Journal of the American Academy of Dermatology 2015;73(1):62-9. [CENTRAL: CN-01085211]

Gupta AK, Hall S, Zane LT, Lipner SR, Rich P. Evaluation of the efficacy and safety of tavaborole topical solution, 5\%, in the treatment of onychomycosis of the toenail in adults: a pooled analysis of an 8-week, post-study follow-up from two randomised phase 3 studies. Journal of Dermatological Treatment 2018;29(1):44-8. [CENTRAL: CN-01458612]

Pariser DM, Wendelken ME, Rycerz AM, Gellings Lowe N, Yost JM, Lipner SR. Planimetric post-hoc analysis of women with onychomycosis from tavaborole $5 \%$ phase III studies: evidence of greater improvements in patients with $>50 \%$ baseline infection. Journal of Drugs in Dermatology 2018;17(2):168-72. [CENTRAL: CN-01627312]

\section{EUCTR2005-005905-51 \{unpublished data only\}}

EUCTR2005-005905-51. A phase II, single-centre, randomised, parallel-group, clinical study to investigate the tolerability of double blind ciclopirox nail product compared to double blind placebo nail product compared to single blind mycoster in subjects with mild to moderate onychomycosis. www.clinicaltrialsregister.eu/ctr-search/ search?query=2005-005905-51 (first received 20 February 2006). [CENTRAL: CN-01813729]

\section{EUCTR2006-000974-54 \{unpublished data only\}}

EUCTR2006-000974-54. A multi-centre, randomized, parallel groups, vehicle and active controlled study of amorolfine $4 \%$ and $10 \%$ nail lacquer new formulation in the topical treatment of distal and lateral subungual toenail onychomycosis. www.clinicaltrialsregister.eu/ctr-search/ search?query=eudract_number:2006-000974-54 (first received 28 February 2007). [CENTRAL: CN-01835701]

\section{EUCTR2006-005895-42 \{unpublished data only\}}

EUCTR2006-005895-42. A study to determine the safety and efficacy of a terbinafine topical formulation system in subjects with onychomycosis in laser treated toenails versus ciclopirox lacquer in intact toenails. www.clinicaltrialsregister.eu/ctrsearch/search?query=2006-005895-42 (first received 2 March 2009). [CENTRAL: CN-01852085]

\section{EUCTR2008-003215-13 \{unpublished data only\}}

EUCTR2008-003215-13. A double-blind, randomized, multicenter, placebo-controlled phase 3 trial to prove the superiority of bifonazole vs. placebo after 4 weeks of onychomycosis treatment (as a follow-up of a 2 weeks treatment of non-surgical nail ablation of diseased nail matrix with a $40 \%$ urea paste). www.clinicaltrialsregister.eu/ctr-search/ search?query=2008-003215-13 (first received 21 October 2008). [CENTRAL: CN-01822662]

\section{EUCTR2016-001242-25 \{unpublished data only\}}

EUCTR2016-001242-25. An early phase development, partly blinded, positive and vehicle controlled, randomized, noninferiority investigation of the pharmacokinetics, safety and efficacy of BB2603 cutaneous hand-pump spray versus Lamisil ${ }^{\circledR}$ spray and versus BB2603 vehicle hand-pump spray in subjects with onychomycosis and associated tinea pedis. www.clinicaltrialsregister.eu/ctr-search/search? query=2016-001242-25 (first received 7 February 2017). [CENTRAL: CN-01816797]

\section{Gupta 2000a \{published data only\}}

Gupta AK, Fleckman P, Baran R. Ciclopirox nail lacquer topical solution $8 \%$ in the treatment of toenail onychomycosis. Journal of the American Academy of Dermatology 2000;43(4 Suppl):S70-80. [CENTRAL: CN-00602467]

\section{Gupta 2000b \{published data only\}}

Gupta AK, Fleckman P, Baran R. Ciclopirox nail lacquer topical solution $8 \%$ in the treatment of toenail onychomycosis. Journal of the American Academy of Dermatology 2000;43(4 Suppl):S70-80. [CENTRAL: CN-00602467]

\section{Gupta 2006 \{published data only\}}

Gupta A, Mo J, Lu M. A randomized, controlled, dose-ranging study to assess the efficacy and safety of terbinafine $\mathrm{HCl}$ nail lacquer for onychomycosis. Journal of the American Academy of Dermatology 2006;54(3 Suppl):AB148. [CENTRAL: CN-00602415]

Hartmane 2013 \{published data only (unpublished sought but not used)\}

Hartmane I, Dervenice A, Mailland F, Mikazans I, Frisenda L, Caserini M. Evaluation of safety profile, pharmacokinetics, and clinical benefit of an innovative terbinafine transungual solution (P-3058): A phase I study in patients with mild-tomoderate distal subungual onychomycosis. Journal of the American Academy of Dermatology 2013;68(4 S1):AB105. 
Hollmig 2014 \{published data only\}

Hollmig ST, Rahman Z, Henderson MT, Rotatori RM, Gladstone H, Tang JY. Lack of efficacy with 1064-nm neodymium:yttriumaluminum-garnet laser for the treatment of onychomycosis: a randomized, controlled trial. Journal of the American Academy of Dermatology 2014;70(5):911-7. [CENTRAL: CN-00988910]

ljzerman 2010 \{published data only (unpublished sought but not used)\}

ljzerman M, Baker J, Flack M, Robinson P. Forty-two-week safety study of topical nanoemulsion (NB-002) for the treatment of mild to moderate distal subungual onychomycosis: a randomized, double-blind, vehicle-controlled trial. Journal of the American Academy of Dermatology 2010;62 (3 Suppl):AB77. [CENTRAL: CN-00843737]

\section{Iorizzo 2016 \{published data only\}}

Iorizzo M, Hartmane I, Derveniece A, Mikazans I. Ciclopirox $8 \% \mathrm{HPCH}$ nail lacquer in the treatment of mild-to-moderate onychomycosis: a randomized, amorolfine controlled study using a blinded evaluator. Skin Appendage Disorders 2016;1(3):134-40. [PUBMED: 27171791]

\section{Karsai 2017 \{published data only\}}

Karsai S, Jager M, Oesterhelt A, Weiss C, Schneider SW, Junger $M$, et al. Treating onychomycosis with the shortpulsed 1064-nm-Nd: YAG laser: results of a prospective randomized controlled trial. Journal of the European Academy of Dermatology and Venereology 2017;31(1):175-80. [CENTRAL: CN-01285896]

\section{Koren 2018 \{published data only\}}

Koren A, Salameh F, Sprecher E, Artzi O. Laser-assisted photodynamic therapy or laser-assisted amorolfine lacquer delivery for treatment of toenail onychomycosis: an openlabel comparative study. Acta Dermato-Venereologia 2018;98(4):467-8. [CENTRAL: CN-01643159]

\section{Kumar 2001 \{published data only\}}

Kumar SV, Kulkarni K. Clinical trial of nailcare capsule and creams in toenail tinea unguium infection. Bombay Hospital Journal 2001;43(4):519-23. [CENTRAL: CN-01094773]

\section{Lahfa 2013 \{published data only\}}

Lahfa M, Bulai-Livideanu C, Baran R, Ortonne J P, Richert B, Tosti A, et al. Efficacy, safety and tolerability of an optimized avulsion technique with onyster ( $40 \%$ urea ointment with plastic dressing) ointment compared to bifonazole-urea ointment for removal of the clinically infected nail in toenail onychomycosis: a randomized evaluator-blinded controlled study. Dermatology 2013;226(1):5-12. [CENTRAL: CN-00906754]

\section{Lu 2005 \{published data only\}}

Lu MQ, Mo J, Bonfrisco J. Randomized, double-blind, parallel design trial to assess the efficacy and safety of terbinafine nail lacquer for the treatment of onychomycosis. Journal of the European Academy of Dermatology and Venereology 2005;19(Suppl 2):394-5. [CENTRAL: CN-00602518]

\section{Montana 1994 \{published data only\}}

Montana JB, Scher RK. A double-blind, vehicle-controlled study of the safety and efficacy of fungoid tincture in patients with distal subungual onychomycosis of the toes. Cutis 1994;53(6):313-6. [CENTRAL: CN-00103913]

NCT01080079 \{unpublished data only\}

NCT01080079. Efficacy and safety study of iontophoretic application of terbinafine gel in subjects with onychomycosis. clinicaltrials.gov/ct2/show/NCT01080079 (first received 3 March 2010). [CENTRAL: CN-01528416]

\section{NCT01145807 \{unpublished data only\}}

NCT01145807. A randomized, double-blind, vehicle- and placebo-controlled, multicenter trial in patients with mild to moderate distal subungual toenail onychomycosis to investigate the efficacy, tolerability, and safety of twice daily application of TDT 067 for 48 weeks. clinicaltrials.gov/ct2/ show/NCT01145807 (first received 17 June 2010). [CENTRAL: CN-01530110]

NCT01246518 \{unpublished data only\}

NCT01246518. Efficacy and safety of two treatment regimens of topical MOB015 in adults with distal subungual onychomycosis. clinicaltrials.gov/ct2/show/NCT01246518 (first received 23 November 2010). [CENTRAL: CN-01502004]

\section{NCT01400594 \{unpublished data only\}}

NCT01400594. Safety and efficacy study of HTU-520 in the treatment of distal subungual onychomycosis of the toenail. clinicaltrials.gov/ct2/show/study/NCT01400594 (first received 22 July 2011). [CENTRAL: CN-01487713]

NCT02022215 \{unpublished data only\}

NCT02022215. Efficacy and safety study of ME1111 in patients with onychomycosis. clinicaltrials.gov/ct2/show/NCT02022215 (first received 27 December 2013). [CENTRAL: CN-01480021]

\section{NCT02343627 \{unpublished data only\}}

NCT02343627. Safety and efficacy of NVXT solution in mild to moderate fungal infection of the toenail. clinicaltrials.gov/ct2/ show/NCT02343627 (first received 22 January 2015). [CENTRAL: CN-01575756]

\section{NCT02933879 \{unpublished data only\}}

NCT02933879. Randomized, placebo-controlled, doubleblind, parallel-group, multi-site phase $2 \mathrm{~b}$ clinical study. www.clinicaltrials.gov/ct2/show/NCT02933879 (first received 14 October 2016). [CENTRAL: CN-01521579]

NCT03141840 \{unpublished data only\} NCT03141840. Clinical trial of topical ABL01 treatment of onychomycosis. www.clinicaltrials.gov/ct2/show/NCT03141840 (first received 5 May 2017). [CENTRAL: CN-01493896]

\section{NCT03289871 \{unpublished data only\}}

NCT03289871. Clinical evaluation of the efficacy of a medical device in treatment of toenail onychomycosis. clinicaltrials.gov/ ct2/show/record/NCT03289871 (first received 21 September 2017). [CENTRAL: CN-01592803] 
Nijenhuis-Rosien 2019 \{published data only (unpublished sought but not used)\}

* Nijenhuis-Rosien L, Kleefstra N, Van Dijk PR, Wolfhagen MJ, Groenier KH, Bilo HJ, et al. Laser therapy for onychomycosis in patients with diabetes at risk for foot ulcers: a randomised, quadruple-blind, sham controlled trial (LASER-1). Journal of the European Academy of Dermatology and Venereology 2019 Mar 28 [Epub ahead of print]. [CENTRAL: CN-01940931; DOI: $10.1111 / j d v .15601]$

Nijenhuis-Rosien L, Kleefstra N, Wolfhagen MJ, Groenier KH, Bilo HJ, Landman GW. Laser therapy for onychomycosis in patients with diabetes at risk for foot complications: study protocol for a randomised, double-blind, controlled trial (LASER-1). Trials 2015;16:108. [CENTRAL: CN-01256326]

\section{Parekh 2017 \{published data only\}}

Parekh M, Ramaiah G, Pashilkar P, Ramanujam R, Johnston P, Ilag LL. A pilot single centre, double blind, placebo controlled, randomized, parallel study of Calmagen ${ }^{\circledR}$ dermaceutical cream and lotion for the topical treatment of tinea and onychomycosis. BMC Complementary and Alternative Medicine 2017;17(1):464. [CENTRAL: CN-01412779; PUBMED: 28923039]

\section{Paul 2013 \{published data only\}}

Paul C, Coustou D, Lahfa M, Bulai-Livideanu C, Doss N, Mokthar I, et al. A multicenter, randomized, open-label, controlled study comparing the efficacy, safety and costeffectiveness of a sequential therapy with RV4104A ointment, ciclopiroxolamine cream and ciclopirox film-forming solution with amorolfine nail lacquer alone in dermatophytic onychomycosis. Dermatology 2013;227(2):157-64. [CENTRAL: CN-01001997]

\section{Romero-Cerecero 2008 \{published data only\}}

Romero-Cerecero O, Zamilpa A, Jimenez-Ferrer JE, RojasBribiesca G, Roman-Ramos R, Tortoriello J. Double-blind clinical trial for evaluating the effectiveness and tolerability of Ageratina pichinchensis extract on patients with mild to moderate onychomycosis. A comparative study with ciclopirox (Planta Med) 2008:October;74(12):1430-1435. Planta Medica 2008;74(14):1767. [CENTRAL: CN-00668953]

\section{Romero-Cerecero 2009 \{published data only\}}

Romero-Cerecero O, Roman-Ramos R, Zamilpa A, JimenezFerrer JE, Rojas-Bribiesca G, Tortoriello J. Clinical trial to compare the effectiveness of two concentrations of the Ageratina pichinchensis extract in the topical treatment of onychomycosis. Journal of Ethnopharmacology 2009;126(1):74-8. [CENTRAL: CN-00733423]

\section{Schalka 2012 \{published data only\}}

Schalka S, Nunes S, Gomes Neto A. Comparative clinical evaluation of efficacy and safety of a formulation containing ciclopirox $8 \%$ in the form of a therapeutic nail lacquer in two different posologies for the treatment of onychomycosis of the toes. Anais Brasileiros de Dermatologia 2012;87(1):19-25. [CENTRAL: CN-00895307]

\section{Sigurgeirsson 2016 \{published data only\}}

Sigurgeirsson B, Ghannoum MA, Osman-Ponchet $\mathrm{H}$, Kerrouche N, Sidou F. Application of cosmetic nail varnish does not affect the antifungal efficacy of amorolfine 5\% nail lacquer in the treatment of distal subungual toenail onychomycosis: results of a randomised active-controlled study and in vitro assays. Mycoses 2016;59(5):319-26. [CENTRAL: CN-01260064]

Stein 2014 \{published data only (unpublished sought but not used)\}

Stein L, Tavakkol A, Wiltz H, Noss M, Pollak R, Kempers S. A blinded review of the ongoing SOLUTION study, a phase $2 b / 3$ study of 2 dosing regimens of a novel $10 \%$ luliconazole solution in patients with onychomycosis. Journal of the American Academy of Dermatology 2014;70(5 Suppl 1):AB88. [DOI: 10.1016/j.jaad.2014.01.367]

\section{Syed 1998 \{published data only\}}

Syed TA, Ahmadpour OA, Ahmad SA, Shamsi S. Management of toenail onychomycosis with $2 \%$ butenafine and $20 \%$ urea cream: a placebo-controlled, double-blind study. Journal of Dermatology 1998;25(10):648-52. [CENTRAL: CN-00157302]

\section{Syed 1999 \{published data only\}}

Syed TA, Qureshi ZA, Ali SM, Ahmad S, Ahmad SA. Treatment of toenail onychomycosis with $2 \%$ butenafine and $5 \%$ Melaleuca alternifolia (tea tree) oil in cream. Tropical Medicine \& International Health 1999;4(4):284-7. [CENTRAL: CN-00164395]

Toledo-Bahena 2014 \{published and unpublished data\}

Toledo-Bahena ME, Bucko A, Ocampo-Candiani J, HerzRuelas ME, Jones TM, Jarratt M T, et al. The efficacy and safety of tavaborole, a novel, boron-based pharmaceutical agent: phase 2 studies conducted for the topical treatment of toenail onychomycosis. Journal of Drugs in Dermatology 2014;13(9):1124-32. [CENTRAL: CN-01113743]

Tschen 2013 \{published data only\}

Tschen EH, Bucko AD, Oizumi N, Kawabata H, Olin JT, Pillai R. Efinaconazole solution in the treatment of toenail onychomycosis: a phase 2, multicenter, randomized, doubleblind study. Journal of Drugs in Dermatology 2013;12(2):186-92. [CENTRAL: CN-01164712]

\section{Waibel 2013 \{published data only\}}

Waibel J, Wulkan A J, Rudnick A. Prospective efficacy and safety evaluation of laser treatments with real-time temperature feedback for fungal onychomycosis. Journal of Drugs in Dermatology 2013;12(11):1237-42. [CENTRAL: CN-00996765]

\section{Watanabe 2017 \{published data only\}}

Watanabe S, Kishida H, Okubo A. Efficacy and safety of luliconazole $5 \%$ nail solution for the treatment of onychomycosis: A multicenter, double-blind, randomized phase III study. Journal of Dermatology 2017;44(7):753-9. [CENTRAL: $\mathrm{CN}-01458932]$ 


\section{References to studies excluded from this review}

\section{Abd El-Aal 2019 \{published data only\}}

Abd El-Aal EB, Abdo HM, Ibrahim SM, Eldestawy MT. Fractional carbon dioxide laser assisted delivery of topical tazarotene versus topical tioconazole in the treatment of onychomycosis. Journal of Dermatological Treatment 2019;30(3):277-82. [PUBMED: 30081698]

\section{Anonymous 2000 \{published data only\}}

Anonymous. Ciclopirox (Penlac) nail lacquer for onychomycosis. Medical Letter on Drugs \& Therapeutics 2000;42(1080):51-2. [PUBMED: 10859733]

\section{Anonymous 2015 \{published data only\}}

Anonymous. Tavaborole topical solution (Kerydin) for onychomycosis. Medical Letter on Drugs \& Therapeutics 2015;57(1463):35-6. [PUBMED: 25719999]

Bassiri-Jahromi 2012 \{published data only (unpublished sought but not used)\}

Bassiri-Jahromi S, Ehsani AH, Mirshams-Shahshahani M, Jamshidi B. A comparative evaluation of combination therapy of fluconazole $1 \%$ and urea $40 \%$ compared with fluconazole $1 \%$ alone in a nail lacquer for treatment of onychomycosis: therapeutic trial. Journal of Dermatological Treatment 2012;23(6):453-6. [CENTRAL: CN-00882643]

\section{Bergstrom 2009 \{published data only\}}

Bergstrom KG. Tea tree oil: Panacea or placebo?. Journal of Drugs in Dermatology 2009;8(5):494-6. [PUBMED: 19537376]

\section{Botter 1971 \{published data only\}}

Botter AA. Topical treatment of nail and skin infections with miconazole, a new broad-spectrum antimycotic. Mykosen 1971;14(4):187-91. [PUBMED: 4251820]

\section{Canavan 2018 \{published data only\}}

Canavan TN, Bevans SL, Cantrell WC, Wang C, Elewski BE. Single-center, prospective, blinded study comparing the efficacy and compatibility of efinaconazole $10 \%$ solution in treating onychomycosis with and without concurrent nail polish use. Skin Appendage Disorders 2018;5(1):9-12. [CENTRAL: CN-01767497; PUBMED: 30643774$]$

\section{Chan 2005 \{published data only\}}

Chan TC, Czarniecki C, Burrows W, Bittker R, Stough DB, Lam GN, et al. EcoNail ${ }^{\mathrm{TM}}$ (5\% econazole plus 18\% SEPA ${ }^{\circledR}$ ) nail lacquer in patients with severe onychomycosis: safety, local tolerability, and systemic exposure. Journal of Investigative Dermatology 2005;125(1):A4. [CENTRAL: CN-00792736]

\section{Dina 2017 \{published data only\}}

Dina A, Gomaa AH, Mohammed GF, Eyada MM, El Sweify MA. Evaluation of the therapeutic efficacy of tea tree oil in treatment of onychomycosis. International Journal of Pharmacognosy and Phytochemical Research 2017;9(12):1414-20. [CENTRAL: $\mathrm{CN}-01466199$ ]
El-Tatawy 2015 \{published data only (unpublished sought but not used)\}

El-Tatawy RA, Abd El-Naby NM, El-Hawary EE, Talaat RA. A comparative clinical and mycological study of Nd-YAG laser versus topical terbinafine in the treatment of onychomycosis. Journal of Dermatological Treatment 2015;26(5):461-4. [CENTRAL: CN-01129437]

Emtestam 2012 \{published data only (unpublished sought but not used)\}

Emtestam L, Kaaman T, Rensfeldt K. Treatment of distal subungual onychomycosis with a topical preparation of urea, propylene glycol and lactic acid: results of a 24-week, doubleblind, placebo-controlled study. Mycoses 2012;55(6):532-40. [CENTRAL: CN-00880045]

Fredriksson 1974 \{published data only\}

Fredriksson T. Topical treatment of superficial mycoses with clotrimazole. Postgraduate Medical Journal 1974;50 Suppl 1:62-4. [CENTRAL: CN-00012751]

Furtado 2005 \{published data only\}

Furtado T, Adjadj L, Halmy K. Cost-effectiveness analysis comparing amorolfine and ciclopirox in the treatment of onychomycosis without matrix involvement. Journal of the American Academy of Dermatology 2005;52:P124.

Gilaberte 2017 \{published data only (unpublished sought but not used)\}

Gilaberte Y, Robres MP, Frias MP, Garcia-Doval I, Rezuta A, Aspiroz C. Methyl aminolevulinate photodynamic therapy for onychomycosis: a multicentre, randomized, controlled clinical trial. Journal of the European Academy of Dermatology and Venereology 2017;31:347-54. [CENTRAL: CN-01332762]

Gupta 2005 \{published data only\}

Gupta AK. Ciclopirox nail lacquer as an adjunct to oral terbinafine for toenail onychomycosis: interim results from a randomized controlled trial. Journal of the European Academy of Dermatology and Venereology 2005;19(Suppl 2):28-9. [CENTRAL: CN-00602229]

Halmy 2005 \{published data only (unpublished sought but not used)\}

Halmy K. Experience with nail lacquers containing amorolfine $5 \%$ and ciclopirox $8 \%$ in patients with onychomycosis. Abstract P-56. 85th BAD Annual Meeting 5-8th July 2005, Glasgow, UK. British Journal of Dermatology 2005;153(Suppl 1):38. [CENTRAL: CN-00527206]

Harris 2010 \{published data only\}

Harris D, Gupta A, Pollak R, Goffe B, Lanzafame R. Pulsed infrared laser treatment for onychomycosis: Controlled, randomized, multi-centered trial $(n=155)$. Lasers in Surgery and Medicine 2010;42:62.

Hay 1988 \{published data only\}

Hay RJ, Roberts DT, Doherty VR, Richardson MD, Midgley G. The topical treatment of onychomycosis using a new combined urea/imidazole preparation. Clinical and Experimental Dermatology 1988;13(3):164-7. [CENTRAL: CN-00240760] 
Jimenez-Faraj 2018 \{published data only\}

Jimenez-Faraj JE, Torres-Oviedo JN, Fiores-Acosta ME, Castillo-Moreno CG, Argueta-Cruz AK, Portillo-Varela EC, et al. Comparison of therapy with $1 \%$ bifonazole plus $40 \%$ urea, monotherapy with $40 \%$ urea and placebo on patients with onychomycosis. Dermatologia Revista Mexicana 2018;62(1):11-8. [CENTRAL: CN-01464271]

\section{Kamalam 1980 \{published data only\}}

Kamalam A, Thambiah AS. Clotrimazole and econazole in dermatophytoses (a double blind study). Mycoses 1980;23(12):707-10. [CENTRAL: CN-00024851]

\section{Kim 2016a \{published data only\}}

Kim MS, Jung JY, Cho EB, Park EJ, Kim KH, Kim KJ. The effectiveness of 1,064-nm long-pulsed Nd:YAG laser in the treatment of severe onychomycosis. Journal of Cosmetic and Laser Therapy 2016;18(6):317-22. [CENTRAL: CN-01208456]

Kim 2016b \{published data only (unpublished sought but not used)\}

Kim TI, Jeong KH, Suh DH, Lee SJ, Shin MK, Lee MH. A randomised comparative study of $1064 \mathrm{~nm}$ neodymium-doped yttrium aluminum garnet ( $\mathrm{Nd}: \mathrm{YAG}$ ) laser and topical antifungal treatment of onychomycosis. Mycoses 2016;59(12):803-10. [CENTRAL: CN-01248276]

\section{Krasaeath 2016 \{published data only\}}

Krasaeath R, Elizondo J. Topical antifungals for treatment of onychomycosis. American Family Physician 2016;94(9):734. [PUBMED: 27929250]

\section{Landsman 2010 \{published data only\}}

Landsman AS, Robbins AH, Angelini PF, Wu CC, Cook J, Oster M, et al. Treatment of mild, moderate, and severe onychomycosis using 870- and 930-nm light exposure. Journal of the American Podiatric Medical Association 2010;100(3):166-77. [CENTRAL: CN-00759930]

\section{Landsman 2012 \{published data only\}}

Landsman AS, Robbins AH. Treatment of mild, moderate, and severe onychomycosis using 870- and 930-nm light exposure: some follow-up observations at 270 days. Journal of the American Podiatric Medical Association 2012;102(2):169-71. [CENTRAL: CN-00903460]

Lauharanta 1992 \{published data only (unpublished sought but not used)\}

Lauharanta J. Comparative efficacy and safety of amorolfine nail lacquer $2 \%$ versus $5 \%$ once weekly. Clinical and Experimental Dermatology 1992;17(Suppl 1):41-3. [CENTRAL: CN-00089244]

Li 2014 \{published data only (unpublished sought but not used)\} Li Y, Yu S, Xu J, Zhang R, Zhao J. Comparison of the efficacy of long-pulsed Nd:YAG laser intervention for treatment of onychomycosis of toenails or fingernails. Journal of Drugs in Dermatology 2014;13(10):1258-63. [CENTRAL: CN-01110964]

\section{Lu 2016 \{published data only\}}

Lu S, Zhang J, Liang Y, Li X, Cai W, Xi L. The efficacy of prognostic factors for long-pulse neodymium: yttrium-aluminum-garnet laser treatment on onychomycosis: A pilot study. Annals of Dermatology 2016;28(3):406-8. [PUBMED: 27274650]

Mahgoub 1975 \{published data only (unpublished sought but not used)\}

Mahgoub ES. Clinical trial with clotrimazole cream (Bay b 5097) in dermatophytosis and onychomycosis. Mycopathologia 1975;56(3):149-52. [PUBMED: 128698]

Malay 2009 \{published data only\}

Malay DS, Yi S, Borowsky P, Downey MS, Mlodzienski AJ. Efficacy of debridement alone versus debridement combined with topical antifungal nail lacquer for the treatment of pedal onychomycosis: a randomized, controlled trial. Journal of Foot \& Ankle Surgery 2009;48(3):294-308. [CENTRAL: CN-00705155]

Many 1964 \{published data only\}

Many H, Franklin CL, Derbes VJ. Candida albicans onychomycosis- a study of five modes of therapy. Dermatologia Tropica Et Ecologica Geographica 1964;3(1):17-20. [CENTRAL: CN-00446588]

Menéndez 2011 \{published data only (unpublished sought but not used)\}

Menéndez S, Falcón L, Maqueira Y. Therapeutic efficacy of topical OLEOZON ${ }^{\circledR}$ in patients suffering from onychomycosis. Mycoses 2011;54(5):e272-7. [PUBMED: 20492527]

\section{Mensing 1992 \{published data only\}}

Mensing H, Polak-Wyss A, Splanemann V. Determination of the subungual antifungal activity of amorolfine after 1 month's treatment in patients with onychomycosis: comparison of two nail lacquer formulations. Clinical and Experimental Dermatology 1992;17(Suppl 1):29-32. [CENTRAL: CN-00089242]

\section{Meyerson 1992 \{published data only\}}

Meyerson MS, Scher RK, Hochman LG, Cohen JL, Pappert AS, Holwell JE. Open-label study of the safety and efficacy of fungoid tincture in patients with distal subungual onychomycosis of the toes. Cutis 1992;49(5):359-62. [CENTRAL: CN-00269422]

\section{Morgado 2017 \{published data only\}}

Morgado LF, Travolo AR, Muehlmann LA, Narcizo PS, Nunes RB, Pereira PA, et al. Photodynamic therapy treatment of onychomycosis with aluminium-phthalocyanine chloride nanoemulsions: A proof of concept clinical trial. Journal of Photochemistry and Photobiology. B, Biology 2017;173:266-70. [PUBMED: 28622558]

\section{NCT03094468 \{unpublished data only\}}

NCT03094468. Study to evaluate the efficacy and safety of $\mathrm{P}-3058$ nail solution in the treatment of onychomycosis. clinicaltrials.gov/ct2/show/NCT03094468 (first received 29 March 2017). 


\section{Oberste-Lehn 1974 \{published data only\}}

Oberste-Lehn $\mathrm{H}$. Ideal properties of a modern antifungal agent-the therapy of mycoses with clotrimazole. Postgraduate Medical Journal 1974;50(Suppl 1):51-3. [PUBMED: 4282870]

\section{Ortiz 2014 \{published data only\}}

Ortiz AE, Truong S, Serowka K, Kelly KM. A 1,320-nm Nd: YAG laser for improving the appearance of onychomycosis. Dermatologic Surgery 2014;40(12):1356-60. [CENTRAL: CN-01037143]

\section{Rand 1996 \{published data only\}}

Rand S. Clinical trials of the new antifungal drug therapies. Journal of the American Academy of Dermatology 1996;34(5 Pt 1):863. [PUBMED: 8632094]

\section{Reinel 1992 \{published data only\}}

Reinel D, Clarke C. Comparative efficacy and safety of amorolfine nail lacquer $5 \%$ in onychomycosis, once-weekly versus twice-weekly. Clinical and Experimental Dermatology 1992;17(Suppl 1):44-9. [CENTRAL: CN-00089245]

\section{Schaller 2017 published data only\}}

Schaller M, Sigurgeirsson B, Sarkany M. Patient-reported outcomes from two randomised studies comparing onceweekly application of amorolfine 5\% nail lacquer to other methods of topical treatment in distal and lateral subungual onychomycosis. Mycoses 2017;60(12):800-7. [CENTRAL: $\mathrm{CN}-01431814]$

\section{Schlockermann 1957 \{published data only\}}

Schlockermann FW. Therapy of onychomycosis. Hautarzt 1957;8(6):270-1. [PUBMED: 13448560]

\section{Shemer 2016 \{published data only\}}

Shemer A, Gupta AK, Amichai B, Farhi R, Baran R, Daniel III CR, et al. An open comparative study of nail drilling as adjunctive treatment for toenail onychomycosis. Journal of Dermatological Treatment 2016;27(5):480-3. [PUBMED: 27032812]

\section{Sigurgeirsson 2010 \{published data only\}}

Sigurgeirsson B, Olafsson JH, Steinsson JT, Kerrouche N, Sidou F. Efficacy of amorolfine nail lacquer for the prophylaxis of onychomycosis over 3 years. Journal of the European Academy of Dermatology and Venereology: JEADV 2010;24(8):910-5. [CENTRAL: CN-00772061]

\section{Sotiriou 2010 \{published data only\}}

Sotiriou E, Koussidou-Eremonti T, Chaidemenos G, Apalla Z, Ioannides D. Photodynamic therapy for distal and lateral subungual toenail onychomycosis caused by Trichophyton rubrum: Preliminary results of a single-centre open trial. Acta Dermato-Venereologica 2010;90(2):216-7. [PUBMED: 20169321]

\section{Souza 2014 \{published data only\}}

Souza LW, Souza SV, Botelho AC. Distal and lateral toenail onychomycosis caused by trichophyton rubrum: treatment with photodynamic therapy based on methylene blue dye. Anais Brasileiros de Dermatologia 2014;89(1):184-6. [PUBMED: 24626676]

\section{Tardivo 2015 \{published data only\}}

Tardivo JP, Wainwright M, Baptista M. Small scale trial of photodynamic treatment of onychomycosis in Sao Paulo. Journal of Photochemistry and Photobiology. B, Biology 2015;150:66-8. [PUBMED: 25835504]

Tietz 2013 \{published data only (unpublished sought but not used)\} Tietz HJ, Hay R, Querner S, Delcker A, Kurka P, Merk HF. Efficacy of 4 weeks topical bifonazole treatment for onychomycosis after nail ablation with $40 \%$ urea: a doubleblind, randomized, placebo-controlled multicenter study. Mycoses 2013;56(4):414-21. [CENTRAL: CN-00964322]

\section{Zaug 1995 \{published data only\}}

Zaug M. Amorolfine nail lacquer: Clinical experience in onychomycosis. Journal of the European Academy of Dermatology and Venereology 1995;4(Suppl 1):S23-30. [CENTRAL: CN-00174369]

Zhang 2012a \{published data only (unpublished sought but not used)\}

Zhang RN, Wang DK, Zhuo FL, Duan XH, Zhang XY, Zhao JY. Long-pulse Nd:YAG 1064-nm laser treatment for onychomycosis. Chinese Medical Journal 2012;125(18):3288-91. [CENTRAL: CN-00967772]

Zhou 2016 \{published data only (unpublished sought but not used)\} Zhou BR, Lu Y, Permatasari F, Huang H, Li J, Liu J, et al. The efficacy of fractional carbon dioxide (CO2) laser combined with luliconazole $1 \%$ cream for the treatment of onychomycosis: A randomized, controlled trial. Medicine 2016;95(44):e5141. [CENTRAL: CN-01306946]

\section{References to studies awaiting assessment}

\section{Anonymous 2002 \{published data only\}}

Anonymous. A frequently underestimated dermatomycosis. Long-term therapeutic success with fungicidal effect. MMW Fortschritte der Medizin 2002;144(43):52-3. [PUBMED: 12534020]

Bagatell 1977 \{published data only\}

Bagatell FK. Topical therapy for onychomycosis. Archives of Dermatology 1977;113(3):378. [CENTRAL: CN-00465913]

\section{Barquero 2007 \{published data only\}}

Barquero N. Treatment of toenail DSO onychomycosis by T rubrum with topical $1 \%$ terbinafine gel. Journal of the American Academy of Dermatology 2007;56(2):AB113. [CENTRAL: $\mathrm{CN}-00616061]$

\section{Butareva 1986 \{published data only\}}

Butareva TA, Kukushkin AM, Unzhakov VP, Nemkaeva RM. 8Hydroxyquinoline derivatives in the treatment of patients with onychomycoses. Vestnik Dermatologii i Venerologii 1986;12:50-3. [PUBMED: 2951932]

\section{Emokpare 1977 \{published data only\}}

Emokpare NA. Clinical evaluation of a new antifungal agent clotrimazole in dermatomycoses. Zeitschrift fur Arztliche Fortbildung (Jena) 1977;71(3):144-6. [PUBMED: 139766] 
EUCTR2008-002427-90 \{unpublished data only\}

EUCTR2008-002427-90. Double-blind study of the clinical and mycological equivalence of Locery ${ }^{\circledR}$ (Amorolfine, filmforming solution at 5\%) and its generic in the treatment of onychomycosis without the nail matrix [Etude en double aveugle de l'équivalence clinique et mycologique du Locéryl ${ }^{\circledR}$ (Amorolfine, solution filmogène à $5 \%$ ) et de son générique dans le traitement des onychomycoses sans atteinte de la matrice unguéale]. www.clinicaltrialsregister.eu/ctr-search/search? query=2008-002427-90 (first received 20 October 2008).

George 1991 \{published data only\}

George MA. A comparison of three treatments for fungal nail infection. British Journal of Podiatric Medicine 1991;46:134-5. [CENTRAL: CN-00252045]

\section{Grant 1974 \{published data only\}}

Grant LV. A further look at the treatment of onychomycosis with topical glutaraldehyde. Journal of the American Podiatry Association 1974;64(3):158-60. [CENTRAL: CN-00250917]

\section{JPRN-JapicCTI-101143 \{unpublished data only\}}

JPRN-JapicCTI-101143. A placebo-controlled, parallel group study of HTU-520 in patients with onychomycosis. apps.who.int/trialsearch/Trial2.aspx?TrialID=JPRNJapicCTI-101143 (first received 24 May 2010).

\section{Klima 1976 \{published data only\}}

Klima J. A report on the clinical experiences from the comparison of the effectiveness of $0.2 \%$ and $0.5 \%$ solutions of the antimycotic agent VUFB 9244 (working name Jopargin). Ceskoslovenska Dermatologie 1976;51(6):404-6. [PUBMED: 137783]

\section{Kull 1972 \{published data only\}}

Kull E. Local treatment of skin and nail mycoses with Daktarin, a new broad-spectrum antifungal agent. Testing results of a group of dermatologists in practice. Schweizerische Rundschau fur Medizin Praxis 1972;61(42):1308-10. [PUBMED: 4263785]

\section{Mathur 1973 \{published data only\}}

Mathur SM, Agarwal RK. Clinical trial of Jadit in superficial mycoses. Indian Journal of Dermatology, Venereology and Leprology 1973;39(6):266-9. [PUBMED: 29139428]

\section{NCT00253305 \{unpublished data only\}}

NCT00253305. Topical gel anti-fungal agent for tinea unguium. clinicaltrials.gov/ct2/show/NCT00253305 (first received 15 November 2005).

\section{NCT02549001 \{unpublished data only\}}

NCT02549001. Study to evaluate the efficacy and safety of P-3058 10\% nail solution in the treatment of toenail onychomycosis. www.clinicaltrials.gov/ct2/show/NCT02549001 (first received 14 September 2015).

\section{Park 2017 \{published data only\}}

Park KY, Suh JH, Kim BJ, Kim MN, Hong CK. Randomized clinical trial to evaluate the efficacy and safety of combination therapy with short-pulsed 1,064-nm neodymium-doped yttrium aluminium garnet laser and amorolfine nail lacquer for onychomycosis. Annals of Dermatology 2017;29(6):699-705. [CENTRAL: CN-01568577; PUBMED: 29200757]

Ramm 1994 \{published data only\}

Ramm S. Loceryl nail lacquer - a new treatment of onychomycosis [Loceryl nagellack - neuer ansatz zur therapie der nagelmykose]. Aktuelle Dermatologie 1994;20(9/10):335-7. [CENTRAL: CN-00727297]

Rüping 1993a \{published data only\}

Ruping KW, Haas PJ. Treatment onychomycoses--bifonazole nail set in comparison with urea with ciclopiroxolamine formulation [Behandlung von onychomykosen--bifonazol-nagelset im vergleich zur harnstoffrezeptur mit ciclopiroxolamin]. Zeitschrift fur Arztliche Fortbildung 1993;87(5):425-9. [CENTRAL: CN-00094233]

Rüping 1993b \{published data only\}

Rüping KW, Haas PJ. Treatment of onychomycoses. Zeitschrift für Allgemeinmedizin 1993;69(20-21):568-72. [CENTRAL: CN-00257388]

\section{Schubert 1973 \{published data only\}}

Schubert E. Clinical experience in the treatment of nail mycoses with the new antibiotic Bay b5097 (clotrimazol). Zeitschrift fur Hautkrankheiten 1973;48(21):887-91. [PUBMED: 4273270]

\section{Weuta 1972 \{published data only\}}

Weuta H. Clotrimazol cream and solution--clinical assessment in an open trial. Arzneimittel-Forschung 1972;22(8):1295-9. [PUBMED: 4264179]

\section{Xu 2017 \{published data only\}}

Xu TH, Su L, Huang D, Li YH, Gao XH, Chen HD. Clinical efficacy of 0.3-ms pulsed 1,064 nm Nd:YAG laser for onychomycosis. Journal of Clinical Dermatology 2017;46(6):450-4. [CENTRAL: CN-01404845]

\section{Yao 2016 \{published data only\}}

Yao Y, Wang XH. Clinical observation of the effects of focused ultrasound combined traditional Chinese medicine on neurodermatitis in middle and aged patients. Journal of the American Geriatrics Society 2016;64:S338.

\section{References to ongoing studies}

\section{ACTRN12618000131257 \{unpublished data only\}}

ACTRN12618000131257. Phase I, first in human (FIH), randomised, double-blind, placebo-controlled multiple ascending dose study in healthy volunteers with mild to moderate onychomycosis. www.anzctr.org.au/Trial/ Registration/TrialReview.aspx?id=374039 (first received 30 January 2018).

\section{ChiCTR-INR-17013504 \{unpublished data only\}}

ChiCTR-INR-17013504. 2940-nm Er:YAG fractional laser combined with amorolfine hydrochloride and amorolfine hydrochloride for treating onychomycosis: a prospective, randomized, controlled, evaluator-blinded, parallel-group clinical trial. www.chictr.org.cn/showproj.aspx?proj=23130 (accessed 16 April 2018). 
DRKS00007709 \{unpublished data only\}

DRKS00007709. Treatment of onychomycosis with a shortpulsed Nd:YAG Laser (1064nm). apps.who.int/trialsearch/ Trial2.aspx?TrialID=DRKS00007709 (first received 20 January 2015).

\section{EUCTR2016-001204-39 \{unpublished data only\}}

EUCTR2016-001204-39. A multi-centre, randomized, twoarmed, parallel group and evaluator-blinded study of efficacy and safety of topical MOB015B in the treatment of mild to moderate distal subungual onychomycosis (DSO). www.clinicaltrialsregister.eu/ctr-search/search? query=2016-001204-39 (first received 15 September 2016).

\section{EUCTR2016-003784-19 \{unpublished data only\}}

EUCTR2016-003784-19. Phase III, multicentre, randomised, double-blind, parallel-group, clinical trial to evaluate the efficacy and safety of a new medicated nail lacquer for the treatment of toenail fungal infection. www.clinicaltrialsregister.eu/ctr-search/search? query=2016-003784-19 (first received 18 April 2017).

\section{JapicCTI-183840 \{unpublished data only\}}

JapicCTI-183840. A phase II multicenter, randomized, double-blind, vehicle-controlled, parallel-group study to investigate the efficacy and safety of ME1111 for 48 weeks of treatment in patients with mild to moderate onychomycosis. www.clinicaltrials.jp/user/showCteDetailE.jsp? japicld=JapicCTI-183840 (first received 22 January 2018).

\section{NCT02644551 \{unpublished data only\}}

NCT02644551. The efficacy of CELEXT07 in the treatment of toenail onychomycosis. www.clinicaltrials.gov/ct2/show/ NCT02644551 (first received 1 January 2016).

\section{NCT02812043 \{unpublished data only\}}

NCT02812043. Comparison between long-pulsed Nd:YAG, amorolfine and combination treatment in treating nondermatophyte onychomycosis. www.clinicaltrials.gov/ct2/ show/NCT02812043 (first received 23 June 2016).

\section{NCT02859519 \{unpublished data only\}}

NCT02859519. A multi-centre, double-blind, randomized, vehicle-controlled study of efficacy and safety of topical MOB015B in the treatment of mild to moderate distal subungual onychomycosis (DSO). www.clinicaltrials.gov/ct2/show/ NCT02859519 (first received 9 August 2016).

\section{NCT02866032 \{unpublished data only\}}

NCT02866032. Study to evaluate the efficacy and safety of topical MOB015B in the treatment of mild to moderate distal subungual onychomycosis (DSO). www.clinicaltrials.gov/ct2/ show/NCT02866032 (first received 15 August 2016).

\section{NCT02961634 \{unpublished data only\}}

NCT02961634. Study efficacy and safety in comparative use of investigational product adjuvant treatment in onychomycosis. www.clinicaltrials.gov/ct2/show/NCT02961634 (first received 11 November 2016).
NCT03098342 \{unpublished data only\}

NCT03098342. Comparison of efficacy and safety between methylene blue-mediated photodynamic therapy and 5\% amorolfine nail lacquer for toenail onychomycosis treatment. www.clinicaltrials.gov/ct2/show/NCT03098342 (first received 31 March 2017).

NCT03814343 \{unpublished data only\}

NCT03814343. Topical amphotericin B in 30\% dimethylsulphoxide in treating of non-dermatophytes onychomycosis. www.clinicaltrials.gov/ct2/show/NCT03814343 (first received 24 January 2019).

\section{Additional references}

\section{Akkus 2017}

Akkus G, Evran M, Gungor D, Karakas M, Sert M, Tetiker T. Tinea pedis and onychomycosis frequency in diabetes mellitus patients and diabetic foot ulcers. A cross sectionalobservational study. Pakistan Journal of Medical Sciences 2016;32(4):891-5.

\section{Anderson 1983}

Anderson RR, Parrish JA. Selective photothermolysis: precise microsurgery by selective absorption of pulsed radiation. Science 1983;220(4596):524-7. [PUBMED: 6836297 ]

\section{Baran 2008}

Baran R, Hay RJ, Garduno JI. Review of antifungal therapy, part II: treatment rationale, including specific patient populations. Journal of Dermatologic Treatment 2008;19(3):168-75. [PUBMED: 18569273]

\section{Baran 2011}

Baran R. The nail in the elderly. Clinics in Dermatology 2011;29(1):54-60. [PUBMED: 21146733]

\section{Barber 2006}

Barber K, Claveau J, Thomas R. Review of treatment for onychomycosis: consideration for special populations. Journa of Cutaenous Medicine and Surgery 2006;10(6 Suppl 2):S48-53. [DOI: 10.2310/7750.2006.00054]

\section{Bristow 2014}

Bristow IR. The effectiveness of lasers in the treatment of onychomycosis: a systematic review. Journal of Foot and Ankle Research 2014;7:34.

\section{Centre for Evidence-Based Medicine}

Centre for Evidence-Based Medicine. Number needed to treat, 2014. www.cebm.net/number-needed-to-treat-nnt/ (accessed 9 September 2015).

\section{Crowley 2015}

Crowley JJ, Weinberg JM, Wu JJ, Robertson AD, Van Voorhees AS, National Psoriasis Foundation. Treatment of nail psoriasis: best practice recommendations from the medical board of the National Psoriasis Foundation. JAMA Dermatology 2015;151(1):87-94. 


\section{CS-COUSIN}

Cochrane Skin. Core Outcomes Set Initiative (CS-COUSIN). skin.cochrane.org/core-outcomes-set-initiative-csg-cousin (accessed 30 November 2018).

\section{Dermik 2003}

Dermik Laboratories. Penlac Nail Lacquer (ciclopirox) Topical Solution $8 \%$ (Product insert). www.accessdata.fda.gov/ drugsatfda_docs/label/2004/21022s004lbl.pdf (accessed 25 October 2018).

\section{Dijkman 2009}

Dijkman B, Kooistra B, Bhandari M, for the Evidence-Based Surgery Working Group. How to work with a subgroup analysis. Canadian Journal of Surgery 2009;52(6):515-22. [PUBMED: 2792383]

\section{Dwan 2008}

Dwan K, Altman DG, Arnaiz JA, Bloom J, Chan A, Cronin E, et al. Systematic review of the empirical evidence of study publication bias and outcome reporting bias. PLoS One 2008;3(8):e3081. [PUBMED: 18769481]

\section{Döner 2011}

Döner N, Yasar S, Ekmekçi TR. Evaluation of obesity-associated dermatoses in obese and overweight individuals [Obezite ile iliskili dermatozlarin obezlerde ve asiri kilolularda arastirilmasi]. Turkderm 2011;45(3):146-51. [EMBASE: 2011536948]

\section{Elewski 2016}

Elewski BE, Gupta AK, Rosen T, Caldwell BD, Pariser DM, Kircik L, et al. Onychomycosis: Does cure equate to treatment success?. Journal of Drugs in Dermatology 2016;15(5):626-32. [PUBMED: 27168271]

\section{Faergemann 2003}

Faergemann J, Baran R. Epidemiology, clinical presentation and diagnosis of onychomycosis. British Journal of Dermatology, Supp/ 2003;149(65):1-4. [EMBASE: 2003465096]

\section{Fukunaga 2013}

Fukunaga A, Washio K, Ogura K, Taguchi K, Chiyomaru K, Ohno $\mathrm{Y}$, et al. Onychomycosis as a warning sign for peripheral arterial disease. Acta Dermatology Venereology 2013;93(6):747-8.

\section{Galderma 2011}

Galderma. Loceryl Nail Lacquer (amorolfine) (Product insert). medicines.org.uk/emc/files/pil.1411.pdf (accessed 20 December 2019).

\section{GRADEPro [Computer program]}

McMaster University. GRADEpro GDT. McMaster University, 2015.

\section{Gupta 1994}

Gupta AK, Sauder DN, Shear NH. Antifungal agents: an overview. Part II. Journal of the American Academy of Dermatology 1994;30(6):911-933. [EMBASE: 1994177736]

\section{Gupta 1997}

Gupta AK, Lynde CW, Jain HC, Sibbald RG, Elewski BE, Daniel CR 3rd, et al. A higher prevalence of onychomycosis in psoriatics compared with non-psoriatics: a multicentre study. British Journal of Dermatology 1997;136(5):786-9. [EMBASE: 1997197390]

\section{Gupta 2000}

Gupta AK, Taborda P, Taborda V, Gilmour J, Rachlis A, Salit I, et al. Epidemiology and prevalence of onychomycosis in HIVpositive individuals. International Journal of Dermatology 2000;39(10):746-53. [EMBASE: 2000387514]

\section{Gupta 2012}

Gupta AK, Drummond-Main C, Cooper EA, Brintnell W, Piraccini BM, Tosti A. Systematic review of nondermatophyte mold onychomycosis: diagnosis, clinical types, epidemiology, and treatment. Journal of the American Academy of Dermatology 2012;66(3):494-502. [EMBASE: 2012113486]

\section{Gupta 2013}

Gupta AK, Paquet M, Simpson FC. Therapies for the treatment of onychomycosis. Clinics in Dermatology 2013;31(5):544-54. [EMBASE: 2013610881]

\section{Gupta 2014a}

Gupta AK, Simpson FC. Investigational drugs for onychomycosis. Expert Opinion on Investigational Drugs 2014;23(1):97-106. [EMBASE: 2013778604]

\section{Gupta 2014b}

Gupta AK, Daigle D, Foley KA. Topical therapy for toenail onychomycosis: an evidence-based review. American Journal of Clinical Dermatology 2014;15(6):489-502. [EMBASE: 25257931]

\section{Gupta 2015a}

Gupta AK, Sibbald RG, Andriessen A, Belley R, Boroditsky A, Botros M, et al. Toenail onychomycosis-A Canadian approach with a new transungual treatment: Development of a clinical pathway. Journal of Cutaneous Medicine and Surgery 2015;19(5):440-9. [PUBMED: 25857439]

\section{Gupta 2015b}

Gupta AK, Daigle D, Foley KA. Network meta-analysis of onychomycosis treatments. Skin Appendage Disorders 2015;1(2):74-81. [PUBMED: 27170937]

\section{Gupta 2016b}

Gupta AK, Simpson FC, Heller DF. The future of lasers in onychomycosis. Journal of Dermatological Treatment 2016;27(2):167-72. [PUBMED: 26270468]

\section{Gupta 2017}

Gupta AK, Versteeg SG. A critical review of improvement rates for laser therapy used to treat toenail onychomycosis. Journal of the European Academy of Dermatology and Venereology 2017;31(7):1111-8. [PUBMED: 28294418] 


\section{Hay 2011}

Hay RJ, Baran R. Onychomycosis: a proposed revision of the clinical classification. Journal of the American Academy Dermatology 2011;65(6):1219-27. [EMBASE: 2011628916]

\section{Higgins 2011}

Higgins JP, Deeks JJ, Altman DJ. Chapter 16: Special topics in statistics. In: Higgins JP, Green S, editor(s). Cochrane Handbook for Systematic Reviews of Interventions Version 5.1.0 (updated March 2011). The Cochrane Collaboration, 2011. Available from handbook.cochrane.org.

\section{Higgins 2017}

Higgins JP, Altman DG, Sterne JA. editor(s). Chapter 8: Assessing risk of bias in included studies. In: Higgins JPT, Churchill R, Chandler J, Cumpston MS (editors), Cochrane Handbook for Systematic Reviews of Interventions version 5.2.0 (updated June 2017), The Cochrane Collaboration, 2017. Available from www.training.cochrane.org/handbook.

\section{Higgins 2019}

Higgins JP, Thomas J, Chandler J, Cumpston M, Li T, Page MJ, et al. editor(s). Cochrane Handbook for Systematic Reviews of Interventions version 6.0 (updated July 2019). The Cochrane Collaboration, 2019. Available from www.training.cochrane.org/ handbook.

\section{Lipner 2018}

Lipner SR, Scher RK. Part 1: Onychomycosis: Clinical overview and diagnosis. Journal of the American Academy of Dermatology 2018 Jun 27 [Epub ahead of print]. [DOI: 10.1016/ j.jaad.2018.03.062]

\section{Lipner 2019}

Lipner SR, Scher RK. Onychomycosis: treatment and prevention of recurrence. Journal of the American Academy of Dermatology 2019;80(4):853-67. [PUBMED: 29959962]

\section{Ozkan 2013}

Ozkan F, Ozturk P, Ozyurt K, Inci MF, Kalender AM, Bakan B, et al. Frequency of peripheral arterial disease and venous insufficiency in toenail onychomycosis. Journal of Dermatology 2013;40(2):107-10.

\section{Papini 2013}

Papini M, Cicoletti M, Fabrizi V, Landucci P. Skin and nail mycoses in patients with diabetic foot. Giornale Italiano di Dermatologia e Venereologia 2013;148(6):603-8.

\section{Piraccini 2008}

Piraccini BM, Rech G, Tosti A. Photodynamic therapy of onychomycosis caused by Trichophyton rubrum. Journal of the American Academy of Dermatology 2008;59(5 Suppl):S75-6. [EMBASE: 2008471503]

\section{Piraccini 2010}

Piraccini BM, Sisti A, Tosti A. Long-term follow-up of toenail onychomycosis caused by dermatophytes after successful treatment with systemic antifungal agents. Journal of the American Academy of Dermatology 2010;62(3):411-4. [PUBMED: 20159308]

\section{Reich 2011}

Reich A, Szepietowski JC. Health-related quality of life in patients with nail disorders. American Journal of Clinical Dermatology 2011;12(5):313-20. [EMBASE: 2011453579]

\section{Review Manager 2014 [Computer program]}

The Nordic Cochrane Centre, The Cochrane Collaboration. Review Manager (RevMan). Version 5.3. Copenhagen: The Nordic Cochrane Centre, The Cochrane Collaboration, 2014.

\section{Rigopoulos 2017}

Rigopoulos D, Papanagiotou V, Daniel R 3rd, Piraccini BM. Onychomycosis in patients with nail psoriasis: a point to point discussion. Mycoses 2017;60(1):6-10.

\section{Rock 2007}

Rock FL, Mao W, Yaremchuk A, Tukalo M, Crépin T, Zhou H, et al. An antifungal agent inhibits an aminoacyl-tRNA synthetase by trapping tRNA in the editing site. Science 2007;213(5832):1759-61. [EMBASE: 2007311724]

\section{Rosen 2015}

Rosen T, Fallon Friedlander S, Kircik L, Zirwas MJ, Stein Gold L, Bhatia N, et al. Onychomycosis: Epidemiology, diagnosis, and treatment in a changing landscape. Journal of Drugs in Dermatology 2015;14(3):223-28.

\section{Rossaneis 2013}

Rossaneis MA, Haddad MD, Mantovani MF, Marcon SS, Pissinati PS. Foot ulceration in patients with diabetes: a risk analysis. British Journal of Nursing 2017;26(6):S6-S14.

\section{Ryan 2016a}

Ryan R, Hill S. How to GRADE the quality of the evidence. Cochrane Consumers and Communication Group. Version 3.0 December 2016. cccrg.cochrane.org/author-resources.

\section{Ryan 2016b}

Ryan R, Synnot A, Hill S. Describing results. Cochrane Consumer and Communications Group. Version 2.0 December 2016. cccrg.cochrane.org/author-resources.

\section{Schulz 2010}

Schulz KF, Altman DG, Moher D. CONSORT 2010 statement: updated guidelines for reporting parallel group randomised trials. BMJ 2010;340:c332. [PUBMED: 20332509]

\section{Sigurgeirsson 2002}

Sigurgeirsson B, Olafsson JH, Steinsson JB, Paul C, Billstein S, Evans EG. Long-term effectiveness of treatment with terbinafine vs itraconazole in onychomycosis: a 5-year blinded prospective follow-up study. Archives of Dermatology 2002;138(3):353-7.

\section{Sigurgeirsson 2014}

Siguregeirsson B, Baran R. The prevalence of onychomycosis in the global population: a literature study. Journal of the European Academy of Dermatology and Venereology 2014;28(11):1480-91. [EMBASE: 24283696] 


\section{Szepietowski 2006}

Szepietowski JC, Reich A, Garlowska E, Kulig M, Baran E. Factors influencing coexistence of toenail onychomycosis with tinea pedis and other dermatomycoses: a survey of 2761 patients. Archives of Dermatology 2006;42(10):1279-84. [EMBASE: 2006511256]

\section{Tosti 1998}

Tosti A, Piraccini BM, Stinchi C, Colombo MD. Relapses of onychomycosis after successful treatment with systemic antifungals: a three-year follow-up. Dermatology (Basel, Switzerland) 1998;197(2):162-6. [PUBMED: 9732167]

\section{U.S. FDA 2015}

U.S. Food, Drug Administration. Medical Devices and Clinical Trial Design for the Treatment or Improvement in the Appearance of Fungally-Infected Nails - Draft Guidance for Industry and FDA Staff 2015. www.fda.gov/ downloads/MedicalDevices/DeviceRegulationandGuidance/ GuidanceDocuments/UCM431312.pdf (accessed 12 October 2017).

\section{Valeant 2004}

Valeant Bermuda. Penlac ${ }^{\circledR}$ Nail Lacquer (ciclopirox) Topica Solution, $8 \%$. www.accessdata.fda.gov/drugsatfda_docs/ label/2004/21022s004lbl.pdf (accessed 9 September 2015).

\section{Ventura 2017}

Ventura A, Mazzeo M, Gaziano R, Galluzzo M, Bianchi L, Campione $\mathrm{E}$. New insight into the pathogenesis of nail psoriasis and overview of treatment strategies. Drug Design, Development and Therapy 2017;11:2527-35. [PUBMED: 28919705]

\section{Welsh 2010}

Welsh O, Vera-Cabrera L, Welsh E. Onychomycosis. Clinics in Dermatology 2010;28(2):151-9. [EMBASE: 2010196893]

\section{Zaias 1972}

Zaias N. Onychomycosis. Archives of Dermatology 1972;105(2):263-74. [EMBASE: 4258535]

\section{References to other published versions of this review Crawford 2007}

Crawford F, Hollis S. Topical treatments for fungal infections of the skin and nails of the foot. Cochrane Database of Systematic Reviews 2007, Issue 3. [DOI: 10.1002/14651858.CD001434.pub2]

\section{Gupta 2016a}

Gupta AK, Simpson F, Daigle D, Villanueva E, John D, Foley K. Topical and device-based treatments for fungal infections of the toenails. Cochrane Database of Systematic Reviews 2016, Issue 2. [DOI: 10.1002/14651858.CD012093]

* Indicates the major publication for the study

\section{CHARACTERISTICS OF STUDIES}

Characteristics of included studies [ordered by study ID]

\section{ACTRN12614000946617}

\begin{tabular}{|c|c|}
\hline \multirow[t]{8}{*}{ Methods } & RCT (parallel) \\
\hline & Study design: Single blind \\
\hline & Diagnosis mycology: Mycological culture \\
\hline & Big toenail specified: Yes \\
\hline & Statistical comparisons for outcomes: Unknown \\
\hline & Duration of follow-up: Weeks $40 \pm 2$ \\
\hline & Time points of measurements: Weeks $40 \pm 2$ \\
\hline & Location: Royal Hobart Hospital, Tasmania, Australia \\
\hline \multirow[t]{4}{*}{ Participants } & Total n: 93 \\
\hline & Age: $\geq 18$ years old \\
\hline & Sex: Men and women (ratio unknown) \\
\hline & $\begin{array}{l}\text { Inclusion criteria: Entry criteria required a clinical diagnosis of onychomycosis (based on clinical man- } \\
\text { ifestations and positive mycology (mycological culture) involving } \leq 20 \% \text { of the area of one of the great } \\
\text { toenails and a minimum age of } 18\end{array}$ \\
\hline
\end{tabular}


ACTRN12614000946617 (Continued)

Exclusion criteria: The main exclusion criteria were other nail conditions (psoriasis, bacterial infections, contact dermatitis, nail bed tumours, yellow nail syndrome, traumatic onychodystrophies, lichen planus, pachonychia congenita and idiopathic onycholysis) confounding onychomycosis assessment, pregnancy, breast-feeding, and a known history of allergy to essential oils. All participants had a 6week washout period for systemic antifungal therapy and 4 weeks for topical antifungal treatment prior to receiving the study design

Disease duration: Unknown

Comparable at baseline: Unknown

Causative species: Unknown

Number of people lost to follow-up: Unknown

Participant enrolment: August 2007 to January 2009

Interventions

Treatment duration: Kunzea oil twice daily (morning and evening) or nail lacquer once a week for $40 \pm 2$ weeks

Drug in study arm 1: Neat Kunzea oil (100\% steam distilled, produced from the Kunzea ambigua plant), $\mathrm{n}=$ unknown

Drug in study arm 2: Loceryl nail lacquer (containing 5\% amorolfine), $\mathrm{n}=$ unknown

\begin{tabular}{ll}
\hline Outcomes & Mycological cure (negative culture) at weeks $40 \pm 2$ \\
& Clinical severity score graded on a visual analogue scale at weeks $40 \pm 2$ \\
& Change in amount of clear nail (digital photographs) and diseases nail (tracings) at weeks $40 \pm 2$ \\
& Adverse events: not reported \\
\hline Notes & The study was government-funded and affiliated with a university. \\
& Trial completed, no results posted. Email response: Manuscript is currently under review
\end{tabular}

\section{Risk of bias}

Bias Authors' judgement Support for judgement

Random sequence genera- Low risk tion (selection bias)

Quote: "participants were randomly allocated into one of two groups (test or control) using a computer-generated randomisation schedule"

Comment: Specific randomisation method that is suitable was specified

$\begin{array}{ll}\begin{array}{l}\text { Allocation concealment } \\ \text { (selection bias) }\end{array} & \text { Quote: "randomisation was provided by the study sponsor, using an indepen- } \\ & \text { dent statistician" "code was kept secure by a staff member (University of Tas- } \\ \text { mania) who was not part of the research team." }\end{array}$

Comment: Allocation concealment was appropriate

\section{Blinding of participants Low risk} and personnel (perfor-

mance bias)

All outcomes
Quote: "Test formulations were prepared in the compounding pharmaceutical laboratory...University of Tasmania, under the supervision of a registered pharmacist...study medications were packed in identical containers...university staff to send the medications (via express registered post) directly to the patients...enabled the clinical investigator (performing assessments) and other study personnel to be blinded."

Comment: Blinding was adequate 
ACTRN12614000946617 (Continued)

Blinding of outcome as- Low risk Quote: "university staff to send the medications (via express registered post) sessment (detection bias) directly to the patients...enabled the clinical investigator (performing assessAll outcomes ments) and other study personnel to be blinded."

Comment: Blinding was adequate

Incomplete outcome data Unclear risk Comment: No information provided in protocol
(attrition bias)

(attrition bias)

All outcomes

Selective reporting (re- Low risk Comment: Outcomes provided in clinical trial register are defined
porting bias)

porting bias

Other bias Unclear risk Comment: Multiple treatment arm study; however, there are no results to assess

\section{ACTRN12614001238662}

Methods

RCT (parallel)

Study design: Unknown

Sample size calculation: Unknown

Diagnosis mycology: Unknown

Big toenail specified: Yes

Statistical comparisons for outcomes: Unknown

Duration of follow-up: At 4 months

Time points of measurement: Months 1 and 4

Location: College of Dentistry/Hawler Medical University, Erbil, Kurdistann, Iraq

Participants

Total n: 75

Age: 18 - 75 years old

Sex: Men and women (ratio unknown)

Inclusion criteria: 18 to 75 years of age inclusive, diagnosed with onycholysis, onychomycosis or onychorrhexis of the nail, at least one target which has: $25 \%$ - $75 \%$ involvement of the area of the nail unit (including destroying or missing parts of the nail plate), at least $2 \mathrm{~mm}$ of uninvolved nail growth as measured from the proximal nail fold to the most proximal point to the disease process and with no lunular involvement, nail unit hyperkeratosis at the most distal edge which measures no greater than $3 \mathrm{~mm}$

Exclusion criteria: Any significant disease of the hepatic, renal, endocrine (including diabetes mellitus), immune systems, or other health conditions which could interfere with or confound the results of study assessments, pre-existing chronic foot pain, neurologic deficit or skin disease / injury in treated foot. In the case of female subjects, are pregnant, nursing or planning to become pregnant within the study period. History of clinically significant abnormal laboratory values, including liver function test results equal to or greater than twice the upper limit of normal. Disease-like psoriatic toenails, significant toenail abnormalities/dystrophies, toenail injuries or any other condition in the toenail, which could interfere with study evaluations

Disease duration: Unknown

Comparable at baseline: Unknown 
ACTRN12614001238662 (Continued)

\section{Causative species: Unknown}

Number of people lost to follow-up: Unknown

Participant enrolment: April 2014 - July 2014

\begin{tabular}{ll}
\hline Interventions & Treatment duration: Applied 4 times daily for 1 month \\
& Drug in study arm 1: Mastic paint $(0.1 \mathrm{ml} / \mathrm{site}), \mathrm{n}=$ unknown \\
Drug in study arm 2: Topically-applied glycerin $(0.1 \mathrm{ml} / \mathrm{site}), \mathrm{n}=$ unknown
\end{tabular}

\begin{tabular}{ll}
\hline Outcomes & $\begin{array}{l}\text { Mycological cure (KOH and culture) at months } 1 \text { and } 4 \\
\text { Clinical cure at months } 1 \text { and } 4 \\
\text { Complete cure at months } 1 \text { and } 4 \\
\text { Adverse events: not reported }\end{array}$ \\
\hline Notes & The study is not funded by industry or affiliated with a university \\
Email response: Manuscript is currently in preparation for publication
\end{tabular}

\section{Risk of bias}

\begin{tabular}{|c|c|c|}
\hline Bias & Authors' judgement & Support for judgement \\
\hline $\begin{array}{l}\text { Random sequence genera- } \\
\text { tion (selection bias) }\end{array}$ & Low risk & $\begin{array}{l}\text { Quote: "table created by computer" } \\
\text { Comment: Method of randomisation likely appropriate }\end{array}$ \\
\hline $\begin{array}{l}\text { Allocation concealment } \\
\text { (selection bias) }\end{array}$ & Unclear risk & $\begin{array}{l}\text { Quote: "by numbered containers" } \\
\text { Comment: Not enough information was available }\end{array}$ \\
\hline $\begin{array}{l}\text { Blinding of participants } \\
\text { and personnel (perfor- } \\
\text { mance bias) } \\
\text { All outcomes }\end{array}$ & Unclear risk & Comment: No information provided in protocol \\
\hline $\begin{array}{l}\text { Blinding of outcome as- } \\
\text { sessment (detection bias) } \\
\text { All outcomes }\end{array}$ & Unclear risk & Comment: No information provided in protocol \\
\hline $\begin{array}{l}\text { Incomplete outcome data } \\
\text { (attrition bias) } \\
\text { All outcomes }\end{array}$ & Unclear risk & Comment: No information provided in protocol \\
\hline $\begin{array}{l}\text { Selective reporting (re- } \\
\text { porting bias) }\end{array}$ & High risk & Comment: Clinical outcomes are not defined. Mycological cure is defined \\
\hline Other bias & Unclear risk & $\begin{array}{l}\text { Comment: Multiple treatment arm study; however, there are no results to as- } \\
\text { sess }\end{array}$ \\
\hline
\end{tabular}

Alberdi 2019

Methods RCT (parallel)


Alberdi 2019 (Continued)

\author{
Study design: Open \\ Sample size calculation: No \\ Diagnosis mycology: PAS and culture \\ Big toenail specified: Yes \\ Statistical comparisons for outcomes: Yes \\ Duration of follow-up: 12 weeks \\ Time points of measurements: Weeks 16 and 28 \\ Location: Hospital Clinico San Carlos, Madrid, Spain
}

\title{
Participants
}

Total n: 46

Age: Mean \pm SD: Photodynamic therapy (PDT) $64.4 \pm 17.3$; Intense pulsed light (IPL) $64.1 \pm 11.4$

Sex: PDT: 4 men and 16 women, 3 not stated, lost to follow-up; IPL: 9 men and 11 women, 3 not stated, lost to follow-up

Inclusion criteria: People with any severity of onychomycosis in the first toe, with mycological diagnosis of positive PAS and positive culture. Patients with prior treatment with oral or topical antifungal agents that was unsuccessful, and/or presence of comorbidity and/or poly-medication that could generate risk when combined with systemic antifungal agents, negative to undergo antifungal treatment were enrolled

Exclusion criteria: Under 18 years of age, contraindications for the use of methylene blue, any diseases causing nail abnormalities and would interfere with evaluation, pregnancy, lactation, use of systemic antifungals within 4 - 24 weeks prior to enrolment

Disease duration: 3 - 5 years

Comparable at baseline: Unknown

Causative species: Dermatophytes, non-dermatophyte moulds, and yeast: Trichophyton species $n=15$, T. rubrum $n=13, T$. mentagrophytes $n=6$, Candida species $n=2$, Candida parapsilosis $n=2$, Candida famata $n=1$, Aspergillus fumigatus $n=2$, Rhodotula $n=1$, Microsporum $n=2$, Fusarium $n=1$, Microsporum + Epidermophyton $\mathrm{n}=1$

Number of people lost to follow-up: PDT $n=3 ;$ IPL $n=3$; all 6 participants decided the study was too time-consuming

Trial duration: unknown

Treatment duration: once every 1 - 2 weeks for 8 treatments, approximately 16 weeks; pre-treatment with $40 \%$ urea for $1-7$ days

Intervention in study arm 1: Methylene blue mediated photodynamic therapy, $\mathrm{n}=23$

Intervention in study arm 2: Intense pulsed light laser therapy, $\mathrm{n}=23$

Outcomes

Clinical improvement measured with Onychomycosis Severity Index (OSI) at weeks 16 and 28

Mycological response (negative PAS and negative culture) at weeks 16 and 28

Complete cure (negative PAS and culture, and 100\% growth of healthy nail) at weeks 16 and 28

Adverse events: reported 


\section{Risk of bias}

Bias Authors' judgement Support for judgement

Random sequence genera- Low risk tion (selection bias)

Quote: "randomly allocated into two different treatment groups by means of a computer-generated random list"

Comment: Specific randomisation method that is suitable was specified

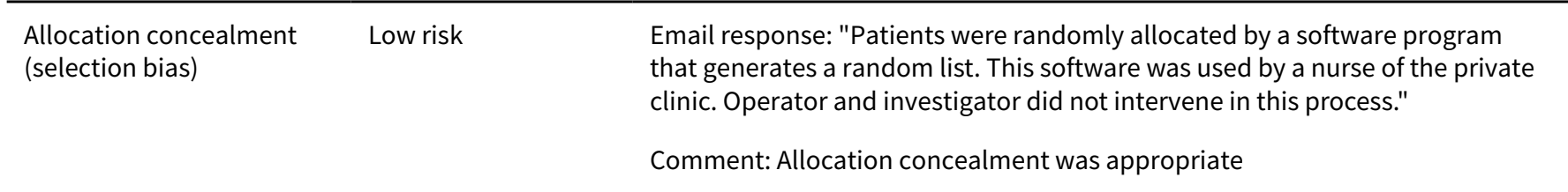

\begin{tabular}{|c|c|c|}
\hline $\begin{array}{l}\text { Blinding of participants } \\
\text { and personnel (perfor- } \\
\text { mance bias) } \\
\text { All outcomes }\end{array}$ & High risk & $\begin{array}{l}\text { Email response: "Participants and operator were not blinded." } \\
\text { Comment: The treatments (photodynamic therapy and intense pulse light) } \\
\text { are very different and it is very likely that participants and personnel were not } \\
\text { blinded }\end{array}$ \\
\hline
\end{tabular}

Blinding of outcome as- High risk sessment (detection bias)

All outcomes
Email response: "the nail treated by methylene blue remained blue during the experiment."

Comment: It was likely that outcome assessors were aware of treatment

\begin{tabular}{lll}
\hline $\begin{array}{l}\text { Incomplete outcome data } \\
\text { (attrition bias) } \\
\text { All outcomes }\end{array}$ & Low risk & $\begin{array}{l}\text { Comment: Reasons for patients discontinuing the study were provided for } \\
\text { each treatment group. The ITT population was clearly specified }\end{array}$ \\
\hline $\begin{array}{l}\text { Selective reporting (re- } \\
\text { porting bias) }\end{array}$ & Low risk & $\begin{array}{l}\text { Email response: Study was not registered in a clinical trial register } \\
\text { Comment: Although not registered, the outcomes specified in Method are re- } \\
\text { ported in Results }\end{array}$ \\
\hline Other bias & Low risk & $\begin{array}{l}\text { Comment: Not a multiple intervention trial, outcomes reported for interven- } \\
\text { tions }\end{array}$ \\
\end{tabular}

Amichai 2010

$\begin{array}{ll}\text { Methods } & \text { RCT (parallel) } \\ \text { Study design: Not blinded } \\ \text { Sample size calculation: No } \\ \text { Diagnosis mycology: KOH and culture } \\ \text { Big toenail specified: Yes } \\ \text { Statistical comparisons for outcomes: Yes } \\ \text { Duration of follow-up: At } 8 \text { and } 12 \text { weeks } \\ \text { Time points of measurements: Weeks 2, 4, } 8 \text { and } 12 \\ \text { Location: Israel (city/institution not stated) }\end{array}$


Amichai 2010 (Continued)
Participants
Total $n=38$
Age: (range): At least $18(20$ - 60) years
Sex: men $22 / 38$ (58\%), women $16 / 38(42 \%)$
Inclusion criteria: Otherwise healthy people, aged at least 18 years, with distal and lateral subungual onychomycosis that involved $25 \%-75 \%$ of the big toenail, caused by dermatophyte
Exclusion criteria: People with matrix involvement, dermatophytoma or yellow streaks were excluded
Disease duration: Unknown
Comparable at baseline: Unknown
Causative species: Dermatophytes
Number of people lost to follow-up: Protocol violations $n=2$; follow-up $n=1$; treatment groups un- known
Trial duration: unknown
Interventions
Treatment duration: Treatment period was overnight wear ( 6 - 8 hours), every day, 5 days a week, for 4 weeks
Drug in study arm 1: $1 \%$ terbinafine $\mathrm{HCl}$ and an iontophoretic patch (current density of $100 \mathrm{uA} / \mathrm{cm} 2), \mathrm{n}$ $=20$
Drug in study arm 2: $1 \%$ terbinafine $\mathrm{HCl}$ patch without iontophoresis, $\mathrm{n}=18$

Outcomes Clinical improvement of the target nail (outgrowth of at least $1.5 \mathrm{~mm}$ of new clinically-unaffected nail plate) at weeks 2, 4, 8, 12

Mycological cure (negative direct microscopy) at weeks 2, 4, 8, 12

Mycological improvement (reduced number of fungal elements in nail specimen and decreased rate of fungal culture growth during and after treatment) of the target nail at weeks 2, 4, 8, 12

Adverse events: reported

Notes

One of 4 authors is an employee of industry, while author is affiliated with industry (relationship not specified)

\section{Risk of bias}

Bias Authors' judgement Support for judgement

Random sequence genera- Unclear risk
tion (selection bias)

Quote: "Patients enrolled in the study were divided randomly into two groups"

Comment: Method of randomisation not specified

Allocation concealment $\quad$ Unclear risk
(selection bias)

Blinding of participants

High risk

Comment: No information was available. Study appears to be an open study and personnel (performance bias)

All outcomes 
Amichai 2010 (Continued)

Incomplete outcome data High risk Quote: "Two patients did not finish the study due to protocol violations." (attrition bias)

Comment: No information on treatment group of protocol violations. Data in-

All outcomes dicate that 1 additional participant was lost to follow-up; treatment unknown

$\begin{array}{ll}\begin{array}{l}\text { Selective reporting (re- } \\ \text { porting bias) }\end{array} & \begin{array}{l}\text { Comment: Review authors were unable to find a clinical trial register entry for } \\ \text { this study }\end{array}\end{array}$

Low risk

Comment: Not a multiple intervention trial, all outcomes reported for interventions

Auvinen 2015

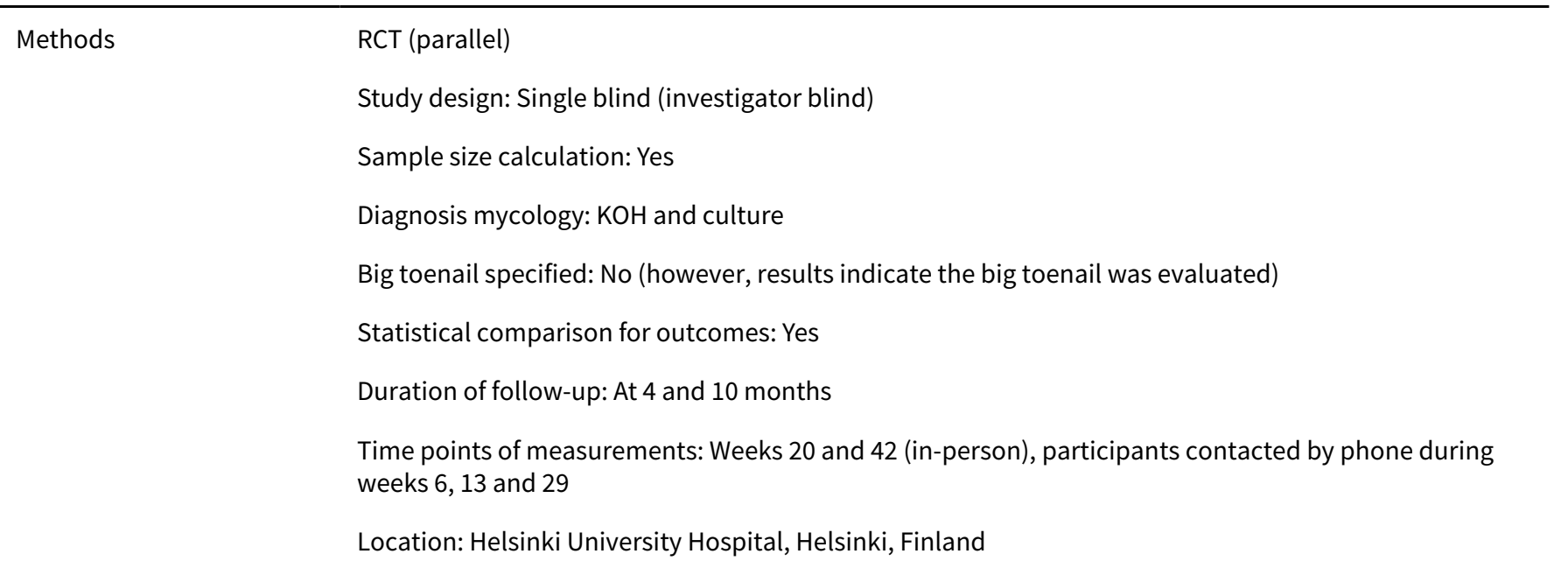

Participants

Total n: 73

Age: Mean \pm SD (range): resin lacquer: $64 \pm 10$ (41 - 87), amorolfine: $63 \pm 11$ (43 - 87), terbinafine: $64 \pm 9$ $(38-77)$

Sex: Resin lacquer: men $21 / 23$ (91\%), women 2/23 (9\%), amorolfine: men 20/25 (80\%), women 5/25 (20\%), terbinafine: men $17 / 25(68 \%)$, women $8 / 25(32 \%)$

Inclusion criteria: Positive dermatophyte culture and a positive $\mathrm{KOH}$ stain

Exclusion criteria: Negative dermatophyte culture or $\mathrm{KOH}$ stain; onychomycosis caused by yeasts or nondermatophyte moulds; liver failure, determined by plasma c-glutamyl transferase levels > $120 \mathrm{U} \mathrm{L} 1$ (2 9 upper limit of normal range); kidney failure, determined by plasma creatinine levels $>100 \mathrm{lmol} L \mathrm{~L}$ (upper limit of normal range); a known hypersensitivity to resin, amorolfine or terbinafine; potential adverse cross-reactions between resin, amorolfine or terbinafine and any concurrently-taken medication; and any prior topical or oral antifungal treatment administered, for any reason, in the year before the beginning of the study

Disease duration: Resin lacquer: 1 - 3 years, 3 participants; 3 - 5 years, 1 participant; 5 - 10 years, 6 participants; 10 years, 13 participants Amorolfine: 1 - 3 years, 4 participants; 3 - 5 years, 3 participants; 5 10 years, 3 participants; 10 years, 15 participants

Terbinafine: 1 - 3 years, 4 participants; 3 - 5 years, 3 participants; 5 - 10 years, 5 participants; 10 years, 13 participants

Comparable at baseline: Yes

Causative species: Resin lacquer: T. rubrum $n=20$ (87\%), T. mentagrophytes $n=3(13 \%)$. Amorolfine: T. rubrum $\mathrm{n}=24(96 \%)$, T. mentagrophytes $\mathrm{n}=1(4 \%)$. Terbinafine: T. rubrum $\mathrm{n}=21(84 \%)$, T. mentagrophytes $n=4(16 \%)$ 
Auvinen 2015 (Continued)

Number of people lost to follow-up: Terbinafine: adverse events $n=2$, participant-based refusal $n=1$.

Amorolfine: subjectively assessed lack of efficacy $n=1$

Participant enrolment: Began October 2013, end date not stated, treatment started January 2014

Interventions

Drug in study arm 1: topical resin lacquer (Abicin 30\% nail lacquer), once daily for 9 months, $n=23$

Drug in study arm 2: 5\% amorolfine hydrochloride, once weekly for 9 months, $n=25$

Drug in study arm 3: oral terbinafine $(250 \mathrm{mg})$, daily for 3 months, $n=25$

\begin{tabular}{|c|c|}
\hline \multirow[t]{5}{*}{ Outcomes } & Mycological cure (negative $\mathrm{KOH}$ staining and negative culture) at 4 and 10 months \\
\hline & Clinical cure (normal appearance of the nail) at 4 and 10 months \\
\hline & Participant compliance (self-reporting throughout study) \\
\hline & Cost effectiveness (average treatment cost per participant based on retail price) \\
\hline & Adverse events: reported \\
\hline Notes & $\begin{array}{l}2 \text { authors of } 6 \text { are shareholders in the company developing the investigational product. This company } \\
\text { also provided funding for the study }\end{array}$ \\
\hline
\end{tabular}

\section{Risk of bias}

\begin{tabular}{lll}
\hline Bias & Authors' judgement & Support for judgement \\
\hline $\begin{array}{l}\text { Random sequence genera- } \\
\text { tion (selection bias) }\end{array}$ & Low risk & $\begin{array}{l}\text { Quote: "Randomization was in permuted blocks of six with an allocation ratio } \\
\text { of 1:1:1" } \\
\text { Comment: Specific randomisation method that is suitable was specified }\end{array}$ \\
\hline $\begin{array}{l}\text { Allocation concealment } \\
\text { (selection bias) }\end{array}$ & Low risk & $\begin{array}{l}\text { Quote: "Treatment was concealed from the investigators; therefore, a research } \\
\text { coordinator allocated patients to the three arms according to the randomisa- } \\
\text { tion list, and delivered the treatments to the patients." }\end{array}$ \\
& \begin{tabular}{l} 
Comment: Allocation concealment was appropriate \\
\hline
\end{tabular}
\end{tabular}

Blinding of participants High risk Comment: Participants were not blinded, potentially influencing results
and personnel (perfor-
mance bias)
All outcomes

Blinding of outcome as- Low risk sessment (detection bias) All outcomes

\begin{abstract}
Quote: "This was an investigator-blinded study...The final analysis and assessment of the treatment outcome was carried out in a blinded manner, without the investigators knowing to which treatment arm the patient belonged at any given time."

Comment: Blinding was adequate
\end{abstract}

\begin{tabular}{lll}
\hline $\begin{array}{l}\text { Incomplete outcome data } \\
\text { (attrition bias) } \\
\text { All outcomes }\end{array}$ & Low risk & $\begin{array}{l}\text { Comment: Reasons for participants discontinuing the study were provided for } \\
\text { each treatment group. The ITT population was clearly specified }\end{array}$ \\
\hline $\begin{array}{l}\text { Selective reporting (re- } \\
\text { porting bias) }\end{array}$ & Low risk & $\begin{array}{l}\text { Comment: Outcomes specified in Methods were reported. Outcomes specified } \\
\text { in trial protocol were all reported. NCT0185150 }\end{array}$ \\
\hline Other bias & Low risk & $\begin{array}{l}\text { Comment: Multiple intervention trial, but outcomes for all interventions re- } \\
\text { ported separately }\end{array}$ \\
\hline
\end{tabular}


Study design: 2 arms (P-3051 and placebo) were double-blinded, while the third arm (market reference: ciclopirox) was open label

Sample size calculation: Yes

Diagnosis mycology: $\mathrm{KOH}$ and culture

Big toenail specified: Yes

Statistical comparisons for outcomes: Yes

Duration of follow-up: At 12 weeks

Time points of measurements: Weeks 48,52 , and 60

Location: Italy (sites, $n=6)$, Germany $(n=2)$, France $(n=2)$, Poland $(n=4)$, Latvia $(n=1)$, Czech Republic $(n=8)$

Participants

Total n: 467 randomised participants, 466 participants with associated data (1 participant in the P-3051 group does not have associated data, explanation not stated)

Age: Mean \pm SD: placebo $49.5 \pm 12.43, \mathrm{P}-305149.47 \pm 12.44$, reference $50.38 \pm 11.09$, all participants $49.84 \pm 11.89$

Sex: Placebo: men 26/97 (26.8\%), women 71/97 (73.2\%); P-3051: men 75/181 (41.4\%), women 106/181 (58.6\%); reference: men 70/188 (37.2\%), women $118 / 188$ (62.8\%). All participants: men $36.7 \%$, women $63.3 \%$.

Inclusion criteria: Patients with distal subungual, mild-to-moderate onychomycosis of at least 1 big toenail (target nail) entered the trial. The target nail for efficacy analysis was chosen between the 2 big toenails, as the most affected within the eligibility criteria. According to the protocol, participants had an infected area $\geq 25 \%$ and $\leq 60 \%$ of target nail. Only people with dermatophyte infection, confirmed by both $\mathrm{KOH}$ microscopy and culture, were randomised to treatment.

Exclusion criteria: People with nail psoriasis, who were positive for yeasts or non-dermatophyte moulds on the nail specimen, and/or who were immunosuppressed were excluded. Local treatment of mycotic infections, with localisation other than in the nails, was allowed

Disease duration: Unknown

Comparable at baseline: The 3 groups were similar with respect to sex, age, weight, number of affected toenails, and in causative pathogen

Causative species: Any dermatophyte: $n=466$ (100\%). T. mentagrophytes: placebo $n=97(39.2 \%)$, P-3051 $n=181(36.8 \%)$, ciclopirox $n=188(37.8 \%)$, all participants $n=466(37.7 \%)$. T. rubrum: placebo $n=97(52.6 \%), P-3051 n=181(54.4 \%)$, ciclopirox $n=188(50.5 \%)$, all participants $(n=466)(52.5 \%)$. T. spp: placebo $n=97(5.1 \%), P-3051 n=181(5.5 \%)$, ciclopirox $n=188(6.9 \%)$, all participants $n=466$ (6.0\%). E floccosum: placebo $n=97(1.0 \%), P-3051 n=181(1.6 \%)$, ciclopirox $n=188(2.7 \%)$, all participants $n=466(2.1 \%)$. Other dermatophytes: placebo $n=97(2.1 \%), P-3051 n=181(1.7 \%)$, ciclopirox $n=$ $188(2.1 \%)$, all participants $n=466(1.9 \%)$

Number of people lost to follow-up: $P-3051 n=25$; reference $n=32$; placebo $n=22$

Trial duration: unknown

Drug in study arm 1: P-3051 (ciclopirox $80 \mathrm{mg} / \mathrm{g}$, water, ethanol, hydroxypropyl chitosan, cetyl stearyl alcohol, ethyl acetate), $\mathrm{n}=182$ 
Baran 2009 (Continued)

Drug in study arm 2: Ciclopirox $80 \mathrm{mg} / \mathrm{g}$ (ethyl acetate, isopropyl alcohol, butyl monoester of

poly[methyl vinyl ether/maleic acid] in isopropyl alcohol) (Market reference), $n=188$

Drug in study arm 3: Placebo (matching vehicle of P-3051, water, ethanol, hydroxypropyl chitosan, cetyl stearyl alcohol, ethyl acetate), $\mathrm{n}=97$

\begin{tabular}{ll}
\hline Outcomes & Mycological cure (negative culture) at weeks 48 and 60 \\
Complete cure (negative $\mathrm{KOH}$ and culture, and $100 \%$ growth of healthy nail) at weeks 48 and 60 \\
Responder (negative $\mathrm{KOH}$ and culture, and decrease of diseased nail area to $\leq 10 \%$ (including zero) of \\
total) \\
Growth rate of healthy nail every 12 weeks \\
Adverse events: reported \\
Quote: "The clinical study was 100\% supported by [industry]" and the sponsor "is the holder of all \\
rights related to the study."
\end{tabular}

\section{Risk of bias}

\begin{tabular}{lll}
\hline Bias & Authors' judgement & Support for judgement \\
\hline $\begin{array}{l}\text { Random sequence genera- } \\
\text { tion (selection bias) }\end{array}$ & Low risk & $\begin{array}{l}\text { Quote: "uneven random allocation of treatments by blocks of 5 (2:2:1) was per- } \\
\text { formed" } \\
\text { Comment: Specific randomisation method that is suitable was specified }\end{array}$ \\
\hline $\begin{array}{l}\text { Allocation concealment } \\
\text { (selection bias) }\end{array}$ & Unclear risk & Comment: No information was available \\
\hline
\end{tabular}

Blinding of participants High risk and personnel (performance bias)

All outcomes
Quote: "Placebo was the matching vehicle of P-3051; thus, the treatment was double-blind between the two arms P-3051 and placebo; the third arm (market reference) was open label, both because the appearance of the reference product and the treatment procedure were different [from the other 2 groups]".

Comment: Adequate participant blinding for 2 of 3 treatment groups. Knowledge of the open arm introduces bias

\begin{tabular}{|c|c|c|}
\hline $\begin{array}{l}\text { Blinding of outcome as- } \\
\text { sessment (detection bias) } \\
\text { All outcomes }\end{array}$ & Low risk & $\begin{array}{l}\text { Quote: "The final evaluation of the primary and secondary clinical endpoints } \\
\text { was centrally made in blind by the International Study Coordinator, who acted } \\
\text { as blinded evaluator." }\end{array}$ \\
\hline & & Comment: Blinding was adequate \\
\hline
\end{tabular}

\begin{tabular}{|c|c|c|}
\hline $\begin{array}{l}\text { Incomplete outcome data } \\
\text { (attrition bias) }\end{array}$ & High risk & $\begin{array}{l}\text { Comment: Based on reported cure rates, participants discontinued the study } \\
\text { Reasons for discontinuation are not reported }\end{array}$ \\
\hline
\end{tabular}

Comment: Based on reported cure rates, participants discontinued the study. All outcomes

\begin{tabular}{lll}
\hline $\begin{array}{l}\text { Selective reporting (re- } \\
\text { porting bias) }\end{array}$ & Unclear risk & $\begin{array}{l}\text { Comment: Review authors were unable to find a clinical trial register entry for } \\
\text { this study }\end{array}$ \\
\hline Other bias & Low risk & $\begin{array}{l}\text { Comment: Multiple intervention trial, but outcomes for all interventions re- } \\
\text { ported separately }\end{array}$ \\
\hline
\end{tabular}


Bonhert 2019

\begin{tabular}{|c|c|}
\hline \multirow[t]{9}{*}{ Methods } & $\mathrm{RCT}$ (parallel) \\
\hline & Study design: Open (evaluating investigator blinded) \\
\hline & Sample size calculation: No \\
\hline & Diagnosis mycology: $\mathrm{KOH}$ and culture \\
\hline & Big toenail specified: Yes \\
\hline & Statistical comparisons for outcomes: Yes \\
\hline & Duration of follow-up: At 4 weeks \\
\hline & Time points of measurements: Weeks 48,52 \\
\hline & Location: United States of America (city/institution not stated) \\
\hline \multirow[t]{10}{*}{ Participants } & Total n: 30 \\
\hline & Age: Mean 51 (range 29 - 69) \\
\hline & Sex: unknown (authors state $\operatorname{men}=18$, women $=13$ ) \\
\hline & $\begin{array}{l}\text { Inclusion criteria: Patients } 18-70 \text { years of age with a clinical diagnosis of DLSO affecting at least } 1 \text { great } \\
\text { toenail. Toenail had to be uninfected length of } 3 \mathrm{~mm} \text { or more from the proximal nail fold and } 3 \mathrm{~mm} \text { or } \\
\text { less in thickness. Diagnosis confirmed with positive KOH microscopy and positive culture of dermato- } \\
\text { phytes or mixed dermatophyte/Candida } \leq 42 \text { days before baseline. Contraception and negative preg- } \\
\text { nancy test for women required }\end{array}$ \\
\hline & $\begin{array}{l}\text { Exclusion criteria: Use of laser/light-based fungal therapy within } 3 \text { months, or topical antifungal ther- } \\
\text { apy applied to the foot or nails within } 1 \text { month of study entry. HIV-positive, infection, tinea pedis, im- } \\
\text { munosuppression and liver toxicity, non-dermatophyte infection, and intolerance to efinaconazole } \\
\text { were also excluded }\end{array}$ \\
\hline & Disease duration: Unknown \\
\hline & Comparable at baseline: Clinical index of onychomycosis was similar between the 2 groups \\
\hline & Causative species: Dermatophytes or mixed dermatophyte/Candida \\
\hline & Number of people lost to follow-up: None \\
\hline & Trial duration: unknown \\
\hline \multirow[t]{3}{*}{ Interventions } & $\begin{array}{l}\text { Treatment duration: daily for } 48 \text { weeks, laser treatment every } 4 \text { weeks for } 6 \text { treatments at the begin- } \\
\text { ning of the study in conjunction with daily topical use }\end{array}$ \\
\hline & Drug in study arm 1: Efinaconazole $10 \%$ solution, $\mathrm{n}=15$ \\
\hline & Intervention in study arm 2: Efinaconazole $10 \%$ solution and $1064 \mathrm{~nm} \mathrm{Nd:YAG} \mathrm{laser,} \mathrm{n}=15$ \\
\hline \multirow[t]{5}{*}{ Outcomes } & $\begin{array}{l}\text { Complete cure (negative } \mathrm{KOH} \text { and culture, and } 0 \% \text { nail involvement with no onycholysis and subungual } \\
\text { hyperkeratosis) at week } 52\end{array}$ \\
\hline & Effective treatment (negative $\mathrm{KOH}$ and culture, and $\leq 10 \%$ nail involvement) at week 52 \\
\hline & $\mathrm{KOH}$ and fungal cultures at weeks 48 and 52 \\
\hline & Adverse events: reported \\
\hline & Participant satisfaction \\
\hline
\end{tabular}


Bonhert 2019 (Continued)

Notes

The study was funded by industry. There is no conflict of interest statement for authors. Author affiliations are academic in nature

\section{Risk of bias}

\begin{tabular}{|c|c|c|}
\hline Bias & Authors' judgement & Support for judgement \\
\hline \multirow{2}{*}{$\begin{array}{l}\text { Random sequence genera- } \\
\text { tion (selection bias) }\end{array}$} & \multirow[t]{2}{*}{ Unclear risk } & Quote: "randomised" \\
\hline & & Comment: Method of randomisation not specified \\
\hline $\begin{array}{l}\text { Allocation concealment } \\
\text { (selection bias) }\end{array}$ & Unclear risk & Comment: No information was provided \\
\hline $\begin{array}{l}\text { Blinding of participants } \\
\text { and personnel (perfor- } \\
\text { mance bias) } \\
\text { All outcomes }\end{array}$ & High risk & $\begin{array}{l}\text { Comment: The differences in treatment application (topical vs laser + topical) } \\
\text { indicates that participants and personnel were probably not blinded }\end{array}$ \\
\hline \multirow[t]{2}{*}{$\begin{array}{l}\text { Blinding of outcome as- } \\
\text { sessment (detection bias) } \\
\text { All outcomes }\end{array}$} & \multirow[t]{2}{*}{ Low risk } & $\begin{array}{l}\text { Quote: "evaluating investigator blinded"; "efficacy assessments will be per- } \\
\text { formed on the target nail for each subject by the same blinded evaluating in- } \\
\text { vestigator" }\end{array}$ \\
\hline & & Comment: Blinding was adequate \\
\hline \multirow{2}{*}{$\begin{array}{l}\text { Incomplete outcome data } \\
\text { (attrition bias) } \\
\text { All outcomes }\end{array}$} & \multirow[t]{2}{*}{ Low risk } & $\begin{array}{l}\text { Quote: "All } 30 \text { patients enrolled completed the study and adhered to the proto- } \\
\text { col." }\end{array}$ \\
\hline & & Comment: All participants were accounted for. \\
\hline $\begin{array}{l}\text { Selective reporting (re- } \\
\text { porting bias) }\end{array}$ & High risk & $\begin{array}{l}\text { Comment: Outcomes stated in Methods are not reported in the Results (com- } \\
\text { plete cure, adverse events) }\end{array}$ \\
\hline Other bias & Low risk & $\begin{array}{l}\text { Comment: Not a multiple intervention trial, but outcomes are reported for all } \\
\text { interventions }\end{array}$ \\
\hline
\end{tabular}

\section{Buck 1994}

$\begin{array}{ll}\text { Methods } & \text { RCT (parallel) } \\ \text { Study design: Double blind } \\ \text { Sample size calculation: Yes } \\ \text { Diagnosis mycology: Culture } \\ \text { Big toenail specified: No } \\ \text { Statistical comparisons for outcomes: Yes } \\ \text { Duration of follow-up: At } 3 \text { months } \\ \text { Time points of measurements: Months 1, 3 (with phone calls), } 6 \\ \text { Location: United States of America (city/institution not stated) }\end{array}$

\section{Participants}

\section{Total n: 117}

Age: Average: clotrimazole 59 years, tea tree oil 61 years. 
Buck 1994 (Continued)

Sex: Clotrimazole: men 15/53 (28\%), women 38/53 (72\%). Tea tree oil: men 15/64 (23\%), women 49/64 $(77 \%)$

Inclusion criteria: All patients presenting to 1 of 3 sites between June 1991 and December 1991 with distal subungual toe onychomycosis proven by culture were enrolled

Exclusion criteria: Use of immune-suppressant therapy within the previous 6 months, had used a topical agent on the toenails in the previous 2 weeks, had a history of psoriasis, or had known HIV infection

Disease duration: Nail disease for more than 1 year: clotrimazole 49/53 (92\%), tea tree oil 59/64 (92\%)

Comparable at baseline: Yes.

Causative species: Clotrimazole: T. rubrum $n=41$ (77\%), T. mentagrophytes $n=10$ (19\%). Tea Tree Oil: $T$. rubrum $n=53(83 \%)$, T. mentagrophytes $n=8(13 \%)$. Number of people lost to follow-up: Moved or their telephone had been disconnected: clotrimazole $n=4$; tea tree oil $n=1 ; 4$ additional participants discontinued due to adverse events, treatment allocation unknown

Participant enrolment: June 1991 - December 1991

\begin{tabular}{ll}
\hline Interventions & Treatment duration: Twice daily for 6 months \\
& Drug in study arm 1: $100 \%$ Melaleuca alternifolia (Tea Tree) Oil, $\mathrm{n}=64$ \\
& Drug in study arm 2: $1 \%$ clotrimazole solution, $\mathrm{n}=53$
\end{tabular}

Outcomes Mycological cure (culture) at 6 months

Clinical assessment (recorded as full, partial, or no resolution of nail appearance) at 1, 3, and 6 months

Participant assessment by phone call at 3-month follow-up ("Were symptoms and nail appearance resolved, improved, stayed the same, or worsened?")

Adverse events: reported

Notes Industry provided technical and financial support

\section{Risk of bias}

\begin{tabular}{lll}
\hline Bias & Authors' judgement & Support for judgement \\
\hline $\begin{array}{ll}\text { Random sequence genera- } \\
\text { tion (selection bias) }\end{array}$ & Low risk & $\begin{array}{l}\text { Quote: "The treatment groups were randomised by the pharmacy by means of } \\
\text { a computerized random-number generator." }\end{array}$ \\
& & Comment: Specific randomisation method that is suitable was specified
\end{tabular}

\begin{tabular}{|c|c|c|}
\hline $\begin{array}{l}\text { Allocation concealment } \\
\text { (selection bias) }\end{array}$ & Low risk & $\begin{array}{l}\text { Comment: Randomisation was performed by the pharmacy, who then pre- } \\
\text { pared and distributed identical treatments to investigators }\end{array}$ \\
\hline
\end{tabular}

\begin{tabular}{|c|c|c|}
\hline $\begin{array}{l}\text { Blinding of participants } \\
\text { and personnel (perfor- } \\
\text { mance bias) }\end{array}$ & Low risk & $\begin{array}{l}\text { Quote: "...pharmacy filled } 60 \text {-cc standardized bottles with solutions...that } \\
\text { appeared identical...the type of medication was blinded to both patient and } \\
\text { provider" }\end{array}$ \\
\hline All outcomes & & Comment: Blinding was adequate \\
\hline
\end{tabular}

\begin{tabular}{lll}
\hline $\begin{array}{l}\text { Blinding of outcome as- } \\
\text { sessment (detection bias) } \\
\text { All outcomes }\end{array}$ & Unclear risk & $\begin{array}{l}\text { Comment: Blinded, but no specific mention of outcome assessor blinding } \\
\text { method. Unclear if provider and investigator are the same individuals }\end{array}$ \\
\hline $\begin{array}{l}\text { Incomplete outcome data } \\
\text { (attrition bias) }\end{array}$ & High risk & $\begin{array}{l}\text { Comment: Participants lost to follow-up identified by treatment; but partici- } \\
\text { pants lost due to adverse events not specified by treatment }\end{array}$ \\
\hline
\end{tabular}


Buck 1994 (Continued)

Selective reporting (re- Low risk Comment: Outcomes specified in Methods reported in Results. Study preporting bias) dates trial registry in the USA

Other bias

Low risk

Comment: Not a multiple intervention trial, but all outcomes reported for interventions

Eertmans 2018

Methods

RCT (parallel)

Study design: Open (evaluator-blinded)

Sample size calculation: Yes

Diagnosis mycology: $\mathrm{KOH}$

Big toenail specified: Yes

Statistical comparisons for outcomes: Yes

Duration of follow-up: None

Time points of measurements: Days $0,30,60,120,180$

Location: Military Hospital of Tunis, Université Tunis El Manar, Tunis, Tunisia

Participants

Total n: 102

Age: $18+$ years of age

Sex: men $25 / 50$ (50\%) test product, $25 / 49$ (51\%) reference; women $25 / 50$ (50\%) test product, $24 / 49$ $(49 \%)$ reference. 2 and 1 participants were lost to follow up in the test product and reference groups, respectively.

Inclusion criteria: Ability to operate medical device, 18 years of age and older with superficial onychomycosis on at least 1 great toenail or light to moderate disto-lateral onychomycosis without matrix involvement, confirmed with $\mathrm{KOH}$ staining, no systemic antifungal within 6 months of study and no topical antifungal within 3 months of study, use of contraception since at least 12 weeks before study until 1 month after the study.

Exclusion criteria: Enrolment in another clinical trial, pregnant, nursing, or women planning to become pregnant, allergy to product constituents, serious or progressive disease such as diabetes, peripheral circulatory disease, HIV, psoriasis, lichen planus, immunosuppression, other cutaneous disease on the nails

Disease duration: Unknown

Comparable at baseline: Yes

Causative species: Dermatophytes, non-dermatophyte moulds, and yeast; test product: $34 / 50$ positive culture, $94.3 \%$ dermatophytes; reference product: $25 / 49$ positive culture, $88.6 \%$ dermatophytes

Number of people lost to follow-up: test product $n=2$, reference product $n=1$

Trial duration: October 26, 2016 (first patient, first visit) - August 26, 2017 (last patient, last visit)

Interventions

Drug in study arm 1: Test product, an aqueous, acetic acid-based, peelable nail polish, once a day for 180 days, $\mathrm{n}=52$

Drug in study arm 2: Reference product, amorolfine 5\% nail lacquer, once a week for 180 days, $n=50$ 
Eertmans 2018 (Continued)

Outcomes

Percentage of healthy nail as assessed using digital photography versus baseline at days 30,60, 120, 180

Clinical efficacy assessed on a 5-point scale for onycholysis, nail dystrophy, nail discolouration, nail thickening at days $30,60,120,180$

Negative $\mathrm{KOH}$ at day 0,180

Evaluation of product tolerance at days $30,60,120,180$

Quality of life using NailQoL questionnaire at days 60, 180

Subjective evaluation with participant questionnaire at days $30,60,120,180$

Adverse events: tolerance to product and quality of life assessed

\section{Risk of bias}

Bias Authors' judgement Support for judgement

Random sequence genera- Low risk tion (selection bias)
Quote: "Prior to the study, a randomisation list was calculated by an external statistician using SAS software (version 9.4). For this purpose, block randomisation with a block size of two was used."

Comment: Specific randomisation method that is suitable was specified
Email response: "The [randomisation list] was generated by the CRO who performed the clinical trial."

Comment: Allocation concealment appropriate

\section{Blinding of participants High risk} and personnel (performance bias)

All outcomes
Quote: "Discernible differences in the product properties (e.g. different bottle, odour) and in the administration process allowed patients to recognize both trial products."

Email response: Unblinded clinical staff member who was not involved in diagnosis or follow-up provided product to participants

Comment: Participants and personnel were not blinded

Blinding of outcome as- Low risk
sessment (detection bias) All outcomes

Quote: "For each photograph, a blinded dermatologist traced the healthy surface. Next, a second evaluator, also blinded, determined the percentage of healthy surface"
Email response: Assessors of mycology were blinded. Clinical evaluators were not involved with providing product to participants

Comment: A blinded evaluator was used for efficacy outcomes but safety was recorded by unblinded investigators

\begin{tabular}{ll}
\hline $\begin{array}{l}\text { Incomplete outcome data } \\
\text { (attrition bias) }\end{array}$ & Low risk \\
All outcomes & each treatment group. The ITT population was clearly specified
\end{tabular}

Comment: Outcomes reported in trial protocol are explicit. The same outcomes are specified and reported in the publication. NCT03382717 
Eertmans 2018 (Continued)

Other bias Low risk Comment: Not a multiple intervention trial, but all outcomes reported for interventions

Elewski 2013a

$\begin{array}{ll}\text { Methods } & \text { RCT (parallel) } \\ \text { Study design: Double blind } \\ \text { Sample size calculation: Yes } \\ \text { Diagnosis mycology: KOH and culture } \\ \text { Big toenail specified: Yes } \\ \text { Statistical comparisons for outcomes: Yes } \\ \text { Duration of follow-up: At } 4 \text { and } 28 \text { weeks } \\ \text { Time points of measurements: Week } 52 \\ \text { Location: unknown }\end{array}$

Participants

Total n: 1044

Age: Mean \pm SD 24-week treatment: terbinafine nail solution (TNS) $53.7 \pm 12.86$ years; Vehicle $52.7 \pm$ 12.51 years; 48-week treatment: terbinafine nail solution (TNS) $54.0 \pm 11.78$ years; Vehicle $53.9 \pm 12.09$ years

Sex: 24-week treatment: TNS: male 200/259 (77.2\%), female 59/259 (22.8\%). Vehicle: male 190/258 (73.6\%), female 68/258 (26.4\%); 48-week treatment: TNS: male 204/271 (75.3\%), female 67/271 (24.7\%). Vehicle: male 194/256 (75.8\%), female $62 / 256(24.2 \%)$

Inclusion criteria: aged 12 - 75 years with mild-to-moderate toe onychomycosis of the great toenail due to dermatophytes. Mild-to-moderate toe onychomycosis was defined as a toenail involvement of $\geq 25 \%$ to $\leq 75 \%$ without spikes and without matrix (lunula) involvement, and nail infection was confirmed by positive $\mathrm{KOH}$ microscopy and culture of a dermatophyte

Exclusion criteria: nail abnormalities, obscuring appearance of infection-free normal nail (including traumatic or onychogryphotic nail) or in whom the target toenail had $<2 \mathrm{~mm}$ unaffected nail plate length beyond the proximal fold, severe plantar tinea pedis requiring systemic therapy, mixed infections (dermatophyte and non-dermatophyte), dermatophytoma (thick masses of fungal hyphae between the nail plate and nail bed), systemic or topical anti-fungal therapy within 6 months or 3 months, respectively, and those who used any commercial topical nail medication within 1 month. People with severe diabetic foot neuropathy, malignancy, and sensitivity to the study medication

Disease duration: Unknown

Comparable at baseline: Unknown

Causative species: 24-week treatment: TNS: T. rubrum $n=252$ (97.3\%), T. mentagrophytes $\mathrm{n}=5(1.9 \%)$, Other $n=1(0.4 \%)$; Vehicle: $n=237(91.9 \%)$, T. mentagrophytes $n=18(7.0 \%)$, Other $n=2(0.8 \%) .48$ week treatment: TNS: T. rubrum $\mathrm{n}=255(94.1 \%)$, T. mentagrophytes $\mathrm{n}=14(5.2 \%)$; Vehicle: T. rubrum $\mathrm{n}=$ 240 (93.8\%), T. mentagrophytes $n=14$ (5.5\%), Other $1 / 256(0.4 \%)$

Number of people lost to follow-up: 24-week treatment, TNS (45 discontinued): adverse events $(n=1)$, unsatisfactory therapeutic effect $(n=5)$, protocol deviation $(n=3)$, consent withdrawn $(n=17)$, loss to follow-up ( $n=19)$. Vehicle (45 discontinued): adverse events $(n=1)$, serious adverse events $(n=1)$, unsatisfactory therapeutic effect $(n=5)$, protocol deviation $(n=2)$, consent withdrawn $(n=17)$, loss to follow-up ( $n=18)$, death $(n=1)$. 48-week treatment, TNS (48 discontinued): adverse events $(n=1)$, serious adverse events $(n=1)$, unsatisfactory therapeutic effect $(n=4)$, protocol deviation $(n=3)$, consent with- 
Elewski 2013a (Continued)

drawal $(n=18)$, loss to follow-up $(n=20)$, death $(n=1)$. Vehicle (35 discontinued): adverse events $(n=$

1 ), unsatisfactory therapeutic effect $(n=2)$, protocol deviation $(n=1)$, consent withdrawn $(n=15)$, loss

to follow up $(n=15)$, other $(n=1)$

Trial duration: unknown

Drug in study arm 1: Topical terbinafine $\mathrm{HCl}$ Nail Solution (TNS) once daily for 24 weeks, $\mathrm{n}=259$
Drug in study arm 2: Vehicle once daily for 24 weeks, $\mathrm{n}=258$
Drug in study arm 3: Topical terbinafine $\mathrm{HCl}$ Nail Solution (TNS) once daily for 48 weeks, $\mathrm{n}=271$
Drug in study arm 4: Vehicle once daily for 48 weeks, $\mathrm{n}=256$

Outcomes

Complete cure (no residual clinical involvement and negative $\mathrm{KOH}$ and culture) at week 52

Mycologoical cure (negative $\mathrm{KOH}$ microscopy and culture) at week 52

Clinical effectiveness ( $\leq 10 \%$ residual involvement of the nail, negative $\mathrm{KOH}$ and culture) at week 52

Clinical cure (no residual clinical involvement) at week 52

Adverse events: reported

Notes 16 authors are employees of industry, 5 additional authors have ties to industry (e.g., consultant,
investigator) and 4 authors have no conflicts of interest. The study was financially supported by an un-
restricted grant from industry

\section{Risk of bias}

\begin{tabular}{lll}
\hline Bias & Authors' judgement & Support for judgement \\
\hline $\begin{array}{ll}\text { Random sequence genera- } \\
\text { tion (selection bias) }\end{array}$ & Low risk & $\begin{array}{l}\text { Quote: "Subjects were randomised using a validated automated system in } \\
\text { equal ratios." }\end{array}$ \\
& & Comment: Specific randomisation method that is suitable was specified.
\end{tabular}

\begin{tabular}{|c|c|c|}
\hline $\begin{array}{l}\text { Allocation concealment } \\
\text { (selection bias) }\end{array}$ & Unclear risk & Comment: No information was available \\
\hline $\begin{array}{l}\text { Blinding of participants } \\
\text { and personnel (perfor- } \\
\text { mance bias) }\end{array}$ & Low risk & $\begin{array}{l}\text { Quote: "Terbinafine nail solution or vehicle were supplied in identical packag- } \\
\text { ing." }\end{array}$ \\
\hline All outcomes & & Comment: Blinding was adequate \\
\hline
\end{tabular}

\begin{tabular}{|c|c|c|}
\hline $\begin{array}{l}\text { Blinding of outcome as- } \\
\text { sessment (detection bias) } \\
\text { All outcomes }\end{array}$ & Unclear risk & $\begin{array}{l}\text { Comment: Blinded, but no specific mention of outcome assessor blinding } \\
\text { method }\end{array}$ \\
\hline
\end{tabular}

\begin{tabular}{ll}
\hline Incomplete outcome data & Low risk \\
(attrition bias) & $\begin{array}{l}\text { Comment: Reasons for patients discontinuing the study were provided for } \\
\text { each treatment group. The ITT population was clearly specified }\end{array}$
\end{tabular}

\begin{tabular}{lll} 
All outcomes & \\
\hline $\begin{array}{l}\text { Selective reporting (re- } \\
\text { porting bias) }\end{array}$ & Low risk & $\begin{array}{l}\text { Comment: Outcomes reported in trial protocol are explicit. The same out- } \\
\text { comes are specified and reported verbatim in the publication. Clinical cure } \\
\text { was added to the publication as an outcome. NCT00443820 and NCT00443898 }\end{array}$ \\
\hline Other bias & High risk & $\begin{array}{l}\text { Quote: "The } 24 \text { week and } 48 \text { week treatment groups were pooled for data col- } \\
\text { lected up to week 28." }\end{array}$
\end{tabular}




Rethods (parallel)
Study design: Open-label, active-controlled
Sample size calculation: Yes
Diagnosis mycology: KOH microscopy and culture of a dermatophyte
Big toenail specified: Yes
Statistical comparisons for outcomes: Yes
Duration of follow-up: 4 weeks
Time points of measurements: Week 52
Location: unknown

Inclusion criteria: aged $12-75$ years with mild-to-moderate toe onychomycosis of the great toenail due to dermatophytes. Mild-to-moderate toe onychomycosis was defined as a toenail involvement of $\geq 25 \%$ to $\leq 75 \%$ without spikes and without matrix (lunula) involvement, and nail infection was confirmed by positive $\mathrm{KOH}$ microscopy and culture of a dermatophyte

Exclusion criteria: nail abnormalities, obscuring appearance of infection-free normal nail (including traumatic or onychogryphotic nail) or in whom the target toenail had $<2 \mathrm{~mm}$ unaffected nail plate length beyond the proximal fold, severe plantar tinea pedis requiring systemic therapy, mixed infections (dermatophyte and non-dermatophyte), dermatophytoma (thick masses of fungal hyphae between the nail plate and nail bed), systemic or topical anti-fungal therapy within 6 months or 3 months, respectively, and those who used any commercial topical nail medication within 1 month. People with severe diabetic foot neuropathy, malignancy, and sensitivity to the study medication

Disease duration: Unknown

Comparable at baseline: Unknown

Causative species: TNS: T. rubrum 484/507 (95.5\%), T. mentagrophytes 17/507 (3.4\%), Other 6/507 (1.2\%). Amorolfine: T. rubrum 488/522 (93.5\%), T. mentagrophytes 25/522 (4.8\%), Other 9/522 (1.7\%)

Number of people lost to follow-up: TNS (66 discontinued): adverse events $(n=1)$, unsatisfactory therapeutic effect $(n=10)$, protocol deviation $(n=4)$, consent withdrawal $(n=20)$, loss to follow-up $(n=27)$, others ( $n=3)$, missing $(n=1)$. Vehicle (76 discontinued): adverse events $(n=6)$, unsatisfactory therapeutic effect $(n=11)$, protocol deviation $(n=3)$, consent withdrawal $(n=26)$, loss to follow-up $(n=30)$

Trial duration: unknown 
Elewski 2013b (Continued)

Outcomes
Complete cure (no residual clinical involvement and negative $\mathrm{KOH}$ and culture) at week 52

Mycologoical cure (negative $\mathrm{KOH}$ microscopy and culture) at week 52

Clinical effectiveness ( $\leq 10 \%$ residual involvement of the nail, negative $\mathrm{KOH}$ and culture) at week 52

Clinical cure (no residual clinical involvement) at week 52

Adverse events: reported

7 of 16 authors are employees of industry, 5 additional authors have ties to industry (e.g. consultant, investigator) and 4 authors have no conflicts of interest. The study was financially supported by an unrestricted grant from industry

\section{Risk of bias}

\begin{tabular}{|c|c|c|}
\hline Bias & Authors' judgement & Support for judgement \\
\hline \multirow[t]{2}{*}{$\begin{array}{l}\text { Random sequence genera- } \\
\text { tion (selection bias) }\end{array}$} & Low risk & $\begin{array}{l}\text { Quote: "Subjects were randomised using a validated automated system in } \\
\text { equal ratios." }\end{array}$ \\
\hline & & Comment: Specific randomisation method that is suitable was specified \\
\hline $\begin{array}{l}\text { Allocation concealment } \\
\text { (selection bias) }\end{array}$ & Unclear risk & Comment: No information was available \\
\hline $\begin{array}{l}\text { Blinding of participants } \\
\text { and personnel (perfor- } \\
\text { mance bias) } \\
\text { All outcomes }\end{array}$ & High risk & Comment: Participants and personnel were not blinded \\
\hline
\end{tabular}

Blinding of outcome as- High risk Comment: Participants and personnel were not blinded

sessment (detection bias)

All outcomes

Incomplete outcome data Low risk

(attrition bias)

All outcomes
Comment: Reasons for participants discontinuing the study were provided for each treatment group. The ITT population was clearly specified

\begin{tabular}{lll}
\hline $\begin{array}{l}\text { Selective reporting (re- } \\
\text { porting bias) }\end{array}$ & Low risk & $\begin{array}{l}\text { Comment: Outcomes reported in trial protocol are explicit. The same out- } \\
\text { comes are specified and reported verbatim in the publication. Clinical cure } \\
\text { was added to the publication as an outcome. NCT00459537 }\end{array}$ \\
\hline Other bias & Low risk & $\begin{array}{l}\text { Comment: Not a multiple intervention trial, but all outcomes reported for in- } \\
\text { terventions }\end{array}$
\end{tabular}

\section{Elewski $2013 \mathrm{c}$}

\begin{tabular}{ll}
\hline Methods & Study 1 \\
RCT (parallel) & Study design: Double blind \\
& Sample size calculation: No \\
& Diagnosis mycology: $\mathrm{KOH}$ and culture \\
& Big toenail specified: $Y$ Yes \\
\hline
\end{tabular}


Duration of follow-up: At 4 weeks

Time points of measurements: Weeks 12, 24, 36, 48, 52

Locations: United States (sites, $n=34)$, Canada $(n=7)$, Japan $(n=33)$ (cities/institutions not stated)

Age: Mean (range): efinaconazole 52.4 years $(20.0$ - 71.0$)$, vehicle 51.9 years $(18.0-70.0)$

Sex: Efinaconazole: men 489/656 (74.5\%), women 167/656 (25.5\%). Vehicle: men 158/214 (73.8\%), women 56/214 (26.2\%). Total: men 647/870 (74.4\%), women $223 / 870$ (25.6\%)

Inclusion criteria: 18 to 70 years of age, clinical diagnosis of DLSO affecting at least 1 great toenail, target toenail uninfected length $3 \mathrm{~mm}$ or more (from the proximal nailfold), thickness $3 \mathrm{~mm}$ or less, evidence of toenail growth, positive $\mathrm{KOH}$ microscopy result, and culture of dermatophyte or mixed dermatophyte/Candida $\leq 42$ days before baseline. Women of childbearing age were required to use birth control

Exclusion criteria: History of immunosuppression and/or clinical signs indicative of possible immunosuppression, known HIV infection, uncontrolled diabetes mellitus, presence of toenail infection other than dermatophytes, severe moccasin tinea pedis at screening/baseline, any disease/condition that might have caused toenail abnormalities or interfered with the evaluation, and previous target toenail surgery. Patients were not excluded for concomitant drugs that inhibit cytochrome P450 3A4

Disease duration: Unknown

Comparable at baseline: Yes

Causative species: Dermatophtyes or mixed dermatophyte/Candida

Number of people lost to follow-up: Efinaconazole: adverse events $(n=21)$, participant request $(n=31)$, lost to follow-up $(n=20)$, other $(n=9)$ Vehicle: adverse events $(n=1)$, participants request $(n=12)$, protocol violation $(n=1)$, lost to follow-up $(n=11)$, other $(n=2)$

Trial duration: unknown

Drug in study arm 1: Efinaconazole $10 \%$ solution, $n=656$

Drug in study arm 2: Vehicle, $\mathrm{n}=214$

Outcomes Complete cure ( $0 \%$ clinical involvement of target toenail and mycologic cure (negative $\mathrm{KOH}$ and negative culture)) at week 52

Mycological cure (negative $\mathrm{KOH}$ and negative culture) at week 52

Treatment success ( $<10 \%$ clinical involvement of target toenail) at week 52

Complete or almost complete cure ( $\leq 5 \%$ clinical involvement and mycologic cure)

Unaffected toenail growth (from baseline) at week 52

Change in number of affected non-target toenails assessed throughout study

Change in quality of life assessed throughout study

Adverse events: reported 
Elewski 2013c (Continued)

Risk of bias

\begin{tabular}{lll}
\hline Bias & Authors' judgement & Support for judgement \\
\hline $\begin{array}{l}\text { Random sequence genera- } \\
\text { tion (selection bias) }\end{array}$ & Low risk & $\begin{array}{l}\text { Quote: "randomisation number determined by a computer-generated ran- } \\
\text { domisation schedule" }\end{array}$ \\
& & Comment: Specific randomisation method that is suitable was specified
\end{tabular}

\begin{tabular}{ll}
\hline $\begin{array}{l}\text { Allocation concealment } \\
\text { (selection bias) }\end{array}$ & Low risk \\
& $\begin{array}{l}\text { Quote: "Study drugs were provided in a kit containing identical masked bottles } \\
\text { with a randomisation number...Access to the randomisation schedule was per- } \\
\text { mitted after both the database was locked and the study unblinded." }\end{array}$
\end{tabular}

Comment: Allocation concealment appropriate

\begin{tabular}{|c|c|c|}
\hline $\begin{array}{l}\text { Blinding of participants } \\
\text { and personnel (perfor- } \\
\text { mance bias) } \\
\text { All outcomes }\end{array}$ & Low risk & $\begin{array}{l}\text { Quote: "Investigators, sponsor, investigational centre staff, clinical monitors, } \\
\text { patients, and all other study personnel were unaware of study drug assign- } \\
\text { ments to individual patients...If the treatment code was broken, the patient } \\
\text { was discontinued." }\end{array}$ \\
\hline & & Comment: Blinding was adequate \\
\hline
\end{tabular}

Blinding of outcome as- Low risk

All outcomes patients...If the treatment code was broken, the patient was discontinued...A study investigator assessed the outcomes at each visit."

Comment: Blinding was adequate

$\begin{array}{ll}\text { Incomplete outcome data Low risk } & \begin{array}{l}\text { Comment: Reasons for participants discontinuing the study were provided for } \\ \text { (attrition bias) }\end{array}\end{array}$

All outcomes

\begin{tabular}{|c|c|c|}
\hline $\begin{array}{l}\text { Selective reporting (re- } \\
\text { porting bias) }\end{array}$ & High risk & $\begin{array}{l}\text { Comment: Primary outcomes different between trial protocol (clinical cure) } \\
\text { and publication (complete cure); outcomes that do appear in trial protocol } \\
\text { are reported in publication. Secondary outcome not defined and vague in tri- } \\
\text { al protocol (clinical efficacy). Multiple outcomes added to publication that are } \\
\text { not found in trial protocol. NCT01007708 }\end{array}$ \\
\hline
\end{tabular}

Other bias Low risk $\quad \begin{aligned} & \text { Comment: Not a multiple intervention trial, but all outcomes reported for in- } \\ & \text { terventions }\end{aligned}$

\section{Elewski 2013d}

$\begin{array}{ll}\text { Methods } & \text { Study } 2 \\ \text { RCT (parallel) } & \text { Study design: Double blind } \\ & \text { Sample size calculation: No } \\ & \text { Diagnosis mycology: } \mathrm{KOH} \text { and culture } \\ & \text { Big toenail specified: Yes } \\ & \text { Statistical comparison for outcomes: Yes } \\ & \text { Duration of follow-up: At } 4 \text { weeks }\end{array}$


Locations: United States (sites, $n=36)$, Canada $(n=8)$ (cities/institutions not stated)

Participants
Total n: 785

Age; 18 to 70 years of age. Mean (range): Efinaconazole 50.6 years (18.0 - 71.0) , Vehicle 50.7 years (18.0 70.0)

Sex: Efinaconazole: men 464 (80.0\%), women 116 (20.0\%). Vehicle: male 164 (81.6\%), women 37 (18.4\%). Total: male $628(80.4 \%)$, women $153(19.6 \%)$

Inclusion criteria: 18 to 70 years of age, clinical diagnosis of DLSO affecting at least 1 great toenail, target toenail uninfected length $3 \mathrm{~mm}$ or more (from the proximal nailfold), thickness $3 \mathrm{~mm}$ or less, evidence of toenail growth, positive $\mathrm{KOH}$ microscopy result, and culture of dermatophyte or mixed dermatophyte/Candida $\leq 42$ days before baseline. Women of childbearing age were required to use birth control

Exclusion criteria: History of immunosuppression and/or clinical signs indicative of possible immunosuppression, known HIV infection, uncontrolled diabetes mellitus, presence of toenail infection other than dermatophytes, severe moccasin tinea pedis at screening/baseline, any disease/condition that might have caused toenail abnormalities or interfered with the evaluation, and previous target toenail surgery. Patients were not excluded for concomitant drugs that inhibit cytochrome P450 3A4

Disease duration: Unknown

Comparable at baseline: Yes

Causative species: Dermatophtyes or mixed dermatophyte/Candida

Number of people lost to follow-up: Efinaconazole: adverse events $(n=11)$, participant request $(n=36)$, protocol violation $(n=3)$, lost to follow-up $(n=29)$, worsening condition $(n=1)$, other $(n=5)$. Vehicle: participant request $(n=19)$, protocol violation $(n=3)$, lost to follow-up $(n=18)$, pregnancy $(n=1)$, other $(n=1)$

Trial duration: unknown

Interventions

Treatment duration: Self-applied once daily for 48 weeks

Drug in study arm 1: Efinaconazole 10\% solution, $n=583$

Drug in study arm 2: Vehicle, $\mathrm{n}=202$

Outcomes

Complete cure $(0 \%$ clinical involvement of target toenail and mycologic cure (negative $\mathrm{KOH}$ and negative culture)) at week 52

Mycological cure (negative $\mathrm{KOH}$ and negative culture) at week 52

Treatment success ( $<10 \%$ clinical involvement of target toenail) at week 52

Complete or almost complete cure ( $\leq 5 \%$ clinical involvement and mycologic cure)

Unaffected toenail growth (from baseline) at week 52

Change in number of affected non-target toenails assessed throughout study

Change in quality of life assessed throughout study

Adverse events: reported

Notes sultant, investigator) 
Elewski 2013d (Continued)

Risk of bias

\begin{tabular}{lll}
\hline Bias & Authors' judgement & Support for judgement \\
\hline $\begin{array}{l}\text { Random sequence genera- } \\
\text { tion (selection bias) }\end{array}$ & Low risk & $\begin{array}{l}\text { Quote: "randomisation number determined by a computer-generated ran- } \\
\text { domisation schedule" }\end{array}$ \\
& & Comment: Specific randomisation method that is suitable was specified
\end{tabular}

$\begin{array}{ll}\begin{array}{l}\text { Allocation concealment } \\ \text { (selection bias) }\end{array} & \text { Low risk } \\ & \begin{array}{l}\text { Quote: "Study drugs were provided in a kit containing identical masked bottles } \\ \text { with a randomisation number...Access to the randomisation schedule was per- } \\ \text { mitted after both the database was locked and the study unblinded." }\end{array}\end{array}$

Comment: Allocation concealment appropriate

\begin{tabular}{|c|c|c|}
\hline $\begin{array}{l}\text { Blinding of participants } \\
\text { and personnel (perfor- } \\
\text { mance bias) } \\
\text { All outcomes }\end{array}$ & Low risk & $\begin{array}{l}\text { Quote: "Investigators, sponsor, investigational centre staff, clinical monitors, } \\
\text { patients, and all other study personnel were unaware of study drug assign- } \\
\text { ments to individual patients...If the treatment code was broken, the patient } \\
\text { was discontinued." }\end{array}$ \\
\hline & & Comment: Blinding was adequate \\
\hline
\end{tabular}

Blinding of outcome as- Low risk sessment (detection bias)

All outcomes

Quote: "Investigators...were unaware of study drug assignments to individual patients...If the treatment code was broken, the patient was discontinued...A study investigator assessed the outcomes at each visit."

Comment: Blinding was adequate

$\begin{array}{ll}\begin{array}{l}\text { Incomplete outcome data } \\ \text { (attrition bias) }\end{array} & \text { Low risk } \\ \text { All } & \text { each treatment group. The ITT population was clearly specified }\end{array}$

All outcomes each treatment group. The ITT population was clearly specified

\begin{tabular}{|c|c|c|}
\hline $\begin{array}{l}\text { Selective reporting (re- } \\
\text { porting bias) }\end{array}$ & High risk & $\begin{array}{l}\text { Comment: Primary outcomes different between trial protocol (clinical cure) } \\
\text { and publication (complete cure); outcomes that do appear in trial protocol } \\
\text { are reported in publication. Secondary outcome not defined and vague in tri- } \\
\text { al protocol (clinical efficacy). Multiple outcomes added to publication that are } \\
\text { not found in trial protocol. NCT01008033 }\end{array}$ \\
\hline
\end{tabular}

Other bias Low risk $\quad \begin{aligned} & \text { Comment: Not a multiple intervention trial, but all outcomes reported for in- } \\ & \text { terventions }\end{aligned}$

\section{Elewski 2015a}

$\begin{array}{ll}\text { Methods } & \text { Study } 1 \\ \text { RCT (parallel) } & \text { Study design: Double blind } \\ & \text { Sample size calculation: No } \\ & \text { Diagnosis mycology: } \mathrm{KOH} \text { and culture } \\ & \text { Big toenail specified: Yes } \\ & \text { Statistical comparisons for outcomes: Yes } \\ & \text { Duration of follow-up: At } 4 \text { weeks and } 12 \text { weeks }\end{array}$


Elewski 2015a (Continued)

Time points of measurements: Weeks 2, 6, and every 6 weeks thereafter; at week 48, participants who achieved complete cure or treatment success were followed until week 60 (post-study follow-up (PSFU))

Location: United States and Mexico, 27 individual sites (cities/institutions not stated)

\section{Participants}

Total n: 594

Age: Mean \pm SD: tavaborole $53.6 \pm 12.5$, vehicle $53.4 \pm 12.3$

Sex: Tavaborole: men 324/399 (81.2\%); women 75/399 (18.8\%); Vehicle: men 158/194 (81.4\%); women $36 / 194(18.6 \%)$

Inclusion criteria: People 18 years of age or older with distal subungual toenail onychomycosis involving $20 \%$ to $60 \%$ of at least 1 TGT were eligible if they had a positive potassium hydroxide $(\mathrm{KOH})$ wet mount and positive culture for dermatophytes, greater than or equal to 3- $\mathrm{mm}$ clear nail measured from the proximal nail fold to the most proximal visible mycotic border, and distal TGT thickness $3 \mathrm{~mm}$ or less

Exclusion criteria: Patients with proximal subungual or superficial white onychomycosis, severe disease, dermatophytoma, exclusively lateral disease, yellow/brown spikes, co-infection with nondermatophyte fungi, anatomic abnormalities of the toes or toenails, active tinea pedis (involving the sides or back of the foot, interdigital, or plantar) requiring treatment, history of chronic moccasin-type tinea pedis (involving the sides or back of the foot), history of other significant chronic fungal disease, psoriasis, lichen planus, known immunodeficiency, significant peripheral vascular disease, known structural heart disease, or uncontrolled diabetes (haemoglobin A1C 8\%). Patients who used topical antifungals on the toenails within 4 weeks or systemic antifungals within 24 weeks were also excluded. Recent use of other topical agents on the toe or toenails, systemic corticosteroids, or immunomodulatory agents was not permitted

Disease duration: Unknown

Comparable at baseline: Yes

Causative species: Dermatophytes

Number of people lost to follow-up: Tavaborole: participant request $n=24$, lost to follow-up $n=18$, noncompliance $n=2$, adverse events $n=1$, other $n=7$. Vehicle: participant request $n=11$, lost to follow-up $n=5$, noncompliance $n=2$, adverse events $n=2$, other $n=3$

Trial duration: December 2010 - November 2012

Interventions

Treatment duration: Once daily for 48 weeks

Drug in study arm 1: Tavaborole topical solution 5\%, $n=400 ;$ PSFU $n=18$

Drug in study arm 2: Vehicle, $\mathrm{n}=194 ;$ PSFU $\mathrm{n}=2$

Outcomes Mycological cure ( $\mathrm{KOH}$ and culture) at week 52

Complete cure (no clinical evidence of onychomycosis and mycological cure) at week 52 and 60

Complete or almost complete clear nail ( $\leq 10 \%$ nail affected) at week 52

Complete or almost complete clear nail ( $\leq 10 \%$ nail affected) plus mycological cure at week 52 and 60 (treatment success)

Adverse events: reported
2 of 9 authors are employees of industry, while the remaining 7 have ties to industry (e.g. advisor, consultant, investigator). Funded by industry. Disclosures accompanied by a quote: "The authors were fully responsible for the content, editorial decisions, and opinions expressed in the current article. No author received an honorarium related to the development of this manuscript." 
Elewski 2015a (Continued)

Risk of bias

\begin{tabular}{lll}
\hline Bias & Authors' judgement & Support for judgement \\
\hline $\begin{array}{l}\text { Random sequence genera- } \\
\text { tion (selection bias) }\end{array}$ & Low risk & $\begin{array}{l}\text { Email response: "The randomisation (blocks of 6) was stratified by investiga- } \\
\text { tional site in a 2:1 ratio to the following treatment groups: tavaborole or vehi- } \\
\text { cle" } \\
\text { Comment: Specific randomisation method that is suitable was specified }\end{array}$ \\
\hline $\begin{array}{l}\text { Allocation concealment } \\
\text { (selection bias) }\end{array}$ & Low risk & $\begin{array}{l}\text { Email response: "Treatment assignment was accomplished by a computer } \\
\text { generated random sequence implemented through the interactive web ran- } \\
\text { domisation system (IWRS)." } \\
\text { Comment: Allocation concealment appropriate }\end{array}$ \\
\hline
\end{tabular}

$\begin{array}{ll}\begin{array}{l}\text { Blinding of participants } \\ \text { and personnel (perfor- }\end{array} & \text { Low risk } \\ \begin{array}{l}\text { mance bias) } \\ \text { All outcomes }\end{array} & \begin{array}{l}\text { Email response: "Tavaborole or matching vehicle was supplied in amber glass } \\ \text { bottles containing } 10 \mathrm{~mL} \text { of investigational product...neither the Investigator } \\ \text { nor the subject knew the subject's treatment assignment." }\end{array} \\ & \text { Comment: Blinding was adequate }\end{array}$

Blinding of outcome as- Low risk Email response: "...neither the Investigator nor the subject knew the subject's sessment (detection bias) treatment assignment. The Sponsor's staff involved in clinical management, All outcomes data management, and statistical evaluation was to remain blinded until a database lock memo was issued and identification of the analysis populations was agreed upon and documented."

Comment: Blinding was adequate

\begin{tabular}{lll}
\hline $\begin{array}{l}\text { Incomplete outcome data } \\
\text { (attrition bias) } \\
\text { All outcomes }\end{array}$ & Low risk & $\begin{array}{l}\text { Comment: Reasons for participants discontinuing the study were provided for } \\
\text { each treatment group. The ITT population was clearly specified }\end{array}$ \\
\hline $\begin{array}{l}\text { Selective reporting (re- } \\
\text { porting bias) }\end{array}$ & Low risk & $\begin{array}{l}\text { Comment: Outcomes reported in trial protocol are explicit. The same out- } \\
\text { comes are specified and reported verbatim in the publication. One variation of } \\
\text { the secondary outcome was added to the publication that is not stated in the } \\
\text { trial protocol. NCT01302119 }\end{array}$ \\
\hline Other bias & Low risk & $\begin{array}{l}\text { Comment: Not a multiple intervention trial, but all outcomes reported for in- } \\
\text { terventions }\end{array}$ \\
\hline
\end{tabular}

Elewski 2015b

$\begin{array}{ll}\text { Methods } & \text { Study } 2 \\ \text { RCT (parallel) } & \text { Study design: Double blind } \\ & \text { Sample size calculation: No } \\ & \text { Diagnosis mycology: } \mathrm{KOH} \text { and culture } \\ & \text { Big toenail specified: Yes } \\ & \text { Statistical comparisons for outcomes: Yes } \\ & \text { Duration of follow-up: At } 4 \text { weeks }\end{array}$


Elewski 2015b (Continued)

Time points of measurements: Weeks 2, 6 , and every 6 weeks thereafter; at week 48 , patients who achieved complete cure or treatment success were followed until week 60 (post-study follow-up (PSFU))

Location: United States and Canada, 32 individual sites (cities/institutions not stated)

Participants

Total $\mathrm{n}: 604$ randomised

Age: Mean \pm SD: tavaborole $55.5 \pm 11.5$, vehicle $55.4 \pm 11.0$

Sex: Tavaborole: men 323/396 (81.6\%), women 73/396 (18.4\%), 3 participants were excluded because study treatment was not dispensed. Vehicle: men 174/205 (84.9\%), women 31/205 (15.1\%)

Inclusion criteria: People 18 years of age or older with distal subungual toenail onychomycosis involving $20 \%$ to $60 \%$ of at least 1 TGT were eligible if they had a positive $\mathrm{KOH}$ wet mount and positive culture for dermatophytes, $\geq 3 \mathrm{~mm}$ clear nail measured from the proximal nail fold to the most proximal visible mycotic border, and distal TGT thickness $3 \mathrm{~mm}$ or less

Exclusion criteria: People with proximal subungual or superficial white onychomycosis, severe disease, dermatophytoma, exclusively lateral disease, yellow/brown spikes, co-infection with nondermatophyte fungi, anatomic abnormalities of the toes or toenails, active tinea pedis (involving the sides or back of the foot, interdigital, or plantar) requiring treatment, history of chronic moccasin-type tinea pedis (involving the sides or back of the foot), history of other significant chronic fungal disease, psoriasis, lichen planus, known immunodeficiency, significant peripheral vascular disease, known structural heart disease, or uncontrolled diabetes (haemoglobin A1C 8\%). Patients who used topical antifungals on the toenails within 4 weeks or systemic antifungals within 24 weeks were also excluded. Recent use of other topical agents on the toe or toenails, systemic corticosteroids, or immunomodulatory agents was not permitted

Disease duration: Unknown

Comparable at baseline: Yes

Causative species: Dermatophtyes

Number of participants lost to follow-up: tavaborole: participant request $n=30$, lost to follow-up $n=$ 10 , noncompliance $n=4$, adverse events $n=2$, other $n=4$. Vehicle: participant request $n=20$, lost to follow-up $n=4$, noncompliance $n=2$, adverse events $n=1$, other $n=1$

Trial duration: February 2011 - January 2013

Treatment duration: Once daily for 48 weeks

Drug in study arm 1: Tavaborole topical solution 5\%, $n=399 ;$ PSFU $n=31$

Drug in study arm 2: Vehicle, $n=205 ;$ PSFU $n=11$

Outcomes Mycological cure ( $\mathrm{KOH}$ and culture) at week 52

Complete cure (no clinical evidence of onychomycosis and mycological cure) at weeks 52 and 60

Complete or almost complete clear nail ( $\leq 10 \%$ nail affected) at week 52

Complete or almost complete clear nail ( $\leq 10 \%$ nail affected) plus mycological cure at week 52 and 60 (treatment success)

Adverse events: reported

Notes

2 of 9 authors are employees of industry, while the remaining 7 have ties to industry (e.g. advisor, consultant, investigator). Funded by industry. Disclosures accompanied by a quote: "The authors were fully responsible for the content, editorial decisions, and opinions expressed in the current article. No author received an honorarium related to the development of this manuscript." 
Elewski 2015b (Continued)

Risk of bias

\begin{tabular}{lll}
\hline Bias & Authors' judgement & Support for judgement \\
\hline $\begin{array}{l}\text { Random sequence genera- } \\
\text { tion (selection bias) }\end{array}$ & Low risk & $\begin{array}{l}\text { Email response: "The randomisation (blocks of 6) was stratified by investiga- } \\
\text { tional site in a 2:1 ratio to the following treatment groups: tavaborole or vehi- } \\
\text { cle." } \\
\text { Comment: Specific randomisation method that is suitable was specified }\end{array}$ \\
\hline $\begin{array}{l}\text { Allocation concealment } \\
\text { (selection bias) }\end{array}$ & Low risk & $\begin{array}{l}\text { Email response: "Treatment assignment was accomplished by a computer } \\
\text { generated random sequence implemented through the interactive web ran- } \\
\text { domisation system (IWRS)." } \\
\text { Comment: Allocation concealment appropriate }\end{array}$ \\
\hline
\end{tabular}

$\begin{array}{ll}\begin{array}{l}\text { Blinding of participants } \\ \text { and personnel (perfor- }\end{array} & \text { Low risk } \\ \begin{array}{l}\text { mance bias) } \\ \text { All outcomes }\end{array} & \begin{array}{l}\text { Email response: "Tavaborole or matching vehicle was supplied in amber glass } \\ \text { bottles containing } 10 \mathrm{~mL} \text { of investigational product...neither the Investigator } \\ \text { nor the subject knew the subject's treatment assignment." }\end{array} \\ & \text { Comment: Blinding was adequate }\end{array}$

Blinding of outcome as- Low risk

All outcomes treatment assignment. The Sponsor's staff involved in clinical management, data management, and statistical evaluation was to remain blinded until a database lock memo was issued and identification of the analysis populations was agreed upon and documented."

Comment: Blinding was adequate

$\begin{array}{ll}\begin{array}{l}\text { Incomplete outcome data } \\ \text { (attrition bias) }\end{array} & \text { Low risk } \\ \text { Allouch treatment group. The ITT population was clearly specified }\end{array}$

All outcomes

$\begin{array}{ll}\begin{array}{l}\text { Selective reporting (re- } \quad \text { Low risk } \\ \text { porting bias) }\end{array} & \begin{array}{l}\text { Comment: Outcomes reported in trial protocol are explicit. The same out- } \\ \text { comes are specified and reported verbatim in the publication. One variation of } \\ \text { the secondary outcome was added to the publication that is not stated in the } \\ \text { trial protocol. NCT } 01270971\end{array}\end{array}$

\begin{tabular}{ll}
\hline Other bias $\quad$ Low risk & $\begin{array}{l}\text { Comment: Not a multiple intervention trial, but all outcomes reported for in- } \\
\text { terventions }\end{array}$ \\
\hline
\end{tabular}

$\begin{array}{ll}\text { Methods } & \text { RCT (parallel) } \\ \text { Study design: Double blind for } 2 \text { treatments, Single blind for third treatment } \\ \text { Sample size calculation: Unknown } \\ \text { Diagnosis mycology: KOH and culture } \\ \text { Big toenail specified: Yes } \\ \text { Statistical comparisons for outcomes: Unknown } \\ \text { Duration of follow-up: Unknown } \\ \text { Time points of measurements: Week } 12\end{array}$


EUCTR2005-005905-51 (Continued)

Location: Iceland (city/institution not stated)

\begin{tabular}{|c|c|}
\hline \multirow[t]{10}{*}{ Participants } & Total n: 50 \\
\hline & Age: 18 - 70 years \\
\hline & Sex: Men and women (ratio unknown) \\
\hline & $\begin{array}{l}\text { Inclusion criteria: Men and women, } 18 \text { - } 70 \text { years of age inclusive, diagno } \\
\text { tal subungual onychomycosis due to dermatophytes ( } \mathrm{KOH} \text { and culture), } \\
\text { nail with }<74 \% \text { nail involvement }\end{array}$ \\
\hline & $\begin{array}{l}\text { Exclusion criteria: Unacceptable contraception, pregnancy or breastfeec } \\
\text { imal subungual onychomycosis involving the nail matrix, systemic or to } \\
\text { months, history of immunosuppression, peripheral vascular disease, pe } \\
\text { skin disease or allergy/hypersensitivity likely to interfere with study, tria } \\
\text { cosis within } 6 \text { months or use of investigational drug within } 30 \text { days of rec } \\
\text { results at the discretion of the investigator }\end{array}$ \\
\hline & Disease duration: Unknown \\
\hline & Comparable at baseline: Unknown \\
\hline & Causative species: Dermatophyte \\
\hline & Number of people lost to follow-up: Unknown \\
\hline & Trial duration: unknown \\
\hline \multirow[t]{4}{*}{ Interventions } & Treatment duration: once daily for 12 weeks \\
\hline & Drug in study arm 1: Ciclopirox $7.5 \%$ solution, $n=$ unknown \\
\hline & Drug in study arm 2: Mycoster $8 \%$ film-forming solution, $n=$ unknown \\
\hline & Drug in study arm 3: Vehicle lacquer to ciclopirox solution, $n=$ unknown \\
\hline
\end{tabular}

Outcomes

Tolerability as measured by the investigator tolerance assessments (inflammation and scaling) and as assessed by participants (burning and pruritus)

Adverse events: not reported

Percentage of target nail affected compared to baseline

Notes Commercial sponsor. Industry funding

\section{Risk of bias}

\begin{tabular}{lll}
\hline Bias & Authors' judgement & Support for judgement \\
\hline $\begin{array}{l}\text { Random sequence genera- } \\
\text { tion (selection bias) }\end{array}$ & Unclear risk & Quote: "randomised" \\
& & Comment: Method of randomisation not specified \\
\hline $\begin{array}{l}\text { Allocation concealment } \\
\text { (selection bias) }\end{array}$ & Unclear risk & Comment: No information provided in protocol \\
\hline $\begin{array}{l}\text { Blinding of participants } \\
\text { and personnel (perfor- } \\
\text { mance bias) } \\
\text { All outcomes }\end{array}$ & Unclear risk & Comment: No information provided in protocol \\
\hline
\end{tabular}


EUCTR2005-005905-51 (Continued)

Blinding of outcome as- Unclear risk Comment: No information provided in protocol sessment (detection bias)

All outcomes

Incomplete outcome data Unclear risk $\quad$ Comment: No information provided in protocol
(attrition bias)

All outcomes

Selective reporting (re- Low risk Comment: Outcomes provided in clinical trial register are defined

porting bias)

Other bias Unclear risk Comment: Multiple treatment arm study, but there are no results to assess

\section{EUCTR2006-000974-54}

Rethods (parallel)
Study design: Double blind
Sample size calculation: No
Diagnosis mycology: Unknown
Big toenail specified: Yes
Statistical comparisons for outcomes: Yes
Duration of follow-up: At 9 months
Time points of measurements: Days 1, 7, 14, 45, 90, 135, 180, 210 and 270
Location: 13 centres in Bulgaria, Czech Republic, Germany, and Iceland (cities/institutions not stated)

\section{Participants}

Total n: 127

Age: 21 - 65 years.

Sex: Amorolfine-NF 10\%: women 13/31 (41.9\%), men 18/31 (58.1\%). Amorolfine-NF 4\%: women 17/30 (56.7\%), men $13 / 30$ (43.3\%). Loceryl 5\%: women 19/34 (55.9\%), men 15/34 (44.1\%). Vehicle: women 19/32 (59.4\%), men 13/32 (40.6\%). Total: women 68/127 (53.5\%), men 59/127 (46.5\%).

Inclusion criteria: Distal and lateral subungual onychomycosis due to dermatophytes, involving at least 1 great toenail with at least $25 \%$ of the nail surface area affected

Exclusion criteria: Involvement of the proximal third of the nail

Disease duration (years): Amorolfine - NF 10\% mean \pm SD: $7.71 \pm 6.3$, median 5.00 (min, max) $(0.4,20.0)$. Amorofline-NF 4\% mean \pm SD: $7.03 \pm 6.7$ median 5.00 (Min, Max) (0.3, 30.0). Loceryl 5\% Nail Lacquer mean $7.88 \pm 7.7$ median 5.00 (Min, Max) (1.0, 34.0). Vehicle mean \pm SD $6.33 \pm 6.2$ median 4.50 (Min, Max) $(0.3,25.0)$. Total $7.24 \pm 6.7$ median 5.00 (Min, Max) $(0.3,34.0)$

Comparable at baseline: Yes

Causative species: Dermatophyte

Number of people lost to follow-up: Discontinuation - Lack of efficacy: amorolfine-NF $10 \% \mathrm{n}=1$, Loceryl $5 \%$ nail lacquer $n=1$, total $n=2$. Lost to follow-up: amorolfine-NF $10 \% n=1$, amorolfine NF $n=2$, Loceryl $5 \%$ nail lacquer $n=1$. Vehicle $n=1$, total $n=5$. Other amorolfine $N F 10 \% n=1$, vehicle $n=2$, total $n$ $=3$. Participant's request: amorolfine NF $10 \% n=4$, amorolfine NF $4 \% n=1$, Loceryl $5 \%$ nail lacquer $n=$ 2 , vehicle $n=1$, total $n=8$ 


Drug in study arm 1: Amorolfine $10 \%$ lacquer daily for 2 weeks then weekly until 6 months, $\mathrm{n}=31$
Drug in study arm 2: Amorolfine 4\% lacquer daily for 2 weeks then weekly until 6 months, $\mathrm{n}=30$
Drug in study arm 3: Vehicle nail lacquer daily for 2 weeks then weekly until 6 months, $\mathrm{n}=34$
$\begin{aligned} & \text { Drug in study arm 4: Loceryl (amorolfine } 5 \% \text { nail lacquer) daily for } 2 \text { weeks then weekly until } 6 \text { months, } \\ & \mathrm{n}=32\end{aligned}$

\begin{tabular}{ll}
\hline Outcomes & Clinical cure (completely cleared target great toenail) at days 1, 7, 14, 45, 90, 135, 180, 210 and 270 \\
& Mycological cure (negative culture) at days 1, 7, 14, 45, 90, 135, 180, 210 and 270 \\
& Adverse events: reported \\
\hline Notes & Commercial sponsor. Industry funding
\end{tabular}

\section{Risk of bias}

\begin{tabular}{lll}
\hline Bias & Authors' judgement & Support for judgement \\
\hline $\begin{array}{l}\text { Random sequence genera- } \\
\text { tion (selection bias) }\end{array}$ & Unclear risk & Quote: "randomised" \\
& & Comment: Method of randomisation not specified \\
\hline $\begin{array}{l}\text { Allocation concealment } \\
\text { (selection bias) }\end{array}$ & Unclear risk & Comment: No information provided in protocol \\
\hline $\begin{array}{l}\text { Blinding of participants } \\
\text { and personnel (perfor- } \\
\text { mance bias) } \\
\text { All outcomes }\end{array}$ & Unclear risk & Comment: No information provided in protocol \\
\hline
\end{tabular}

\begin{tabular}{lll}
\hline $\begin{array}{l}\text { Blinding of outcome as- } \\
\text { sessment (detection bias) } \\
\text { All outcomes }\end{array}$ & Unclear risk & Comment: No information provided in protocol \\
\hline $\begin{array}{l}\text { Incomplete outcome data } \\
\text { (attrition bias) } \\
\text { All outcomes }\end{array}$ & Low risk & $\begin{array}{l}\text { Comment: Reasons for discontinuation were provided for each treatment } \\
\text { group. The ITT population was clearly specified }\end{array}$ \\
\hline $\begin{array}{l}\text { Selective reporting (re- } \\
\text { porting bias) }\end{array}$ & High risk & $\begin{array}{l}\text { Comment: Results submitted to trial registry limited, with data for analysis not } \\
\text { extractable apart from safety outcomes. Planned statistical comparisons and } \\
\text { reporting of clinical outcomes do not include all treatment groups. No publica- } \\
\text { tion or further data available for cross-reference }\end{array}$ \\
\hline
\end{tabular}

$\begin{array}{ll}\text { Other bias } \quad \text { Low risk } & \begin{array}{l}\text { Comment: Multiple intervention trial, but safety reported for all interventions } \\ \text { and treatment outcomes analysed without pooling of interventions }\end{array}\end{array}$

$\begin{array}{ll}\text { Methods } & \text { RCT (parallel) } \\ & \text { Study design: Open } \\ & \text { Sample size calculation: Unknown }\end{array}$


Diagnosis mycology: $\mathrm{KOH}$ and culture

Big toenail specified: Yes

Statistical comparisons for outcomes: Unknown

Duration of follow-up: 12 weeks

Time points of measurements: Week 48 and 60

Location: Switzerland (city/institution unknown)

Total n: 235
Age: $18-75$ years
Sex: Men and women (ratio unknown)
Inclusion criteria: Men and women, $18-75$ years of age, diagnosed with distal/lateral subungual ony-
chomycosis due to dermatophytes (KOH and culture), involving at least 1 great toenail with $15 \%$ to $75 \%$
nail involvement
Exclusion criteria: Non-dermatophyte infections, pregnant or lactating women, hypersensitivity to
terbinafine or ciclopirox, structural deformities of the toenails, oral antifungals to treat onychomycosis
within 6 months, topical antifungals to treat onychomycosis within 3 months, dermatophytoma
Disease duration: Unknown
Comparable at baseline: Unknown
Causative species: Dermatophyte
Number of people lost to follow-up: Unknown
Trial duration: unknown

Interventions Treatment duration: once daily for 48 weeks

Drug in study arm 1: Laser method to create perforated holes (once) followed by $10 \%$ terbinafine ethanolic solution and $8 \%$ terbinafine lacquer, $n=$ unknown

Drug in study arm 2: ciclopirox $8 \%$ lacquer, $\mathrm{n}=$ unknown

\begin{tabular}{ll}
\hline Outcomes & Complete cure (100\% clear nail and negative $\mathrm{KOH}$ and culture) at week 48 \\
& Mycological cure \\
& Time to complete cure \\
& Effectively treated/almost clear \\
& Relapse (in those achieving complete cure) at week 60 \\
& Adverse events: not reported \\
\hline Notes & Commercial sponsor. Industry funding \\
\hline Risk of bias & Authors' judgement Support for judgement \\
\hline Bias & Unclear risk $\quad$ Quote: "randomised" \\
\hline $\begin{array}{l}\text { Random sequence genera- } \\
\text { tion (selection bias) }\end{array}$ & \begin{tabular}{l} 
Comment: Method of randomisation not specified \\
\hline
\end{tabular}
\end{tabular}


EUCTR2006-005895-42 (Continued)
Allocation concealment
Unclear risk
Comment: No information provided in protocol (selection bias)

Blinding of participants High risk Comment: The study was not blinded
and personnel (perfor-
mance bias)

\begin{tabular}{lll}
\hline Blinding of outcome as- & High risk & Comment: The study was not blinded \\
sessment (detection bias) &
\end{tabular}

sessment (detection bias)

All outcomes

Incomplete outcome data Unclear risk Comment: No information provided in protocol
(attrition bias)

All outcomes

Selective reporting (re- High risk

porting bias)

Comment: Primary outcome defined clearly. Secondary outcomes provided in clinical trial register not defined

Other bias Unclear risk Comment: There are no results to assess

\section{EUCTR2008-003215-13}

\begin{tabular}{ll}
\hline Methods & RCT (parallel) \\
& Study design: Double blind \\
& Sample size calculation: Yes \\
& Diagnosis mycology: microscopy and culture \\
& Big toenail specified: No \\
& Statistical comparisons for outcomes: Yes \\
& Duration of follow-up: At 6 months \\
& Time points of measurements: Day 14, months 3 and 6 \\
& Location: Germany (sites, $n=35)$, Poland $(\mathrm{n}=6)$, Czech Republic $(\mathrm{n}=10)$ \\
\hline
\end{tabular}

\section{Participants}

Total n: 692

Age: At least 18 years

Sex: Men 384/692 (55.5\%), women 308/692 (44.5\%)

Inclusion criteria: Aged at least 18 years with positive clinical and mycological findings of onychomycosis (positive microscopy and positive culture with identification of pathogen). People with psoriasis at head/neck and torso, fontaine stage I (no symptoms), no signs of venous disease or presenting with only ecstatic or reticular veins or with varicose veins (Widmer stage I - II), known rheumatic disease not requiring any treatment, known history of Hepatitis $\mathrm{C}$ in the past not requiring any treatment and basalioma or actinic keratodermia could be included in the study

Exclusion criteria: Nail mycosis in $>3$ nails (each nail not more than $50 \%$ infected area, in the target nail between $20 \%$ and $50 \%$ )

Disease duration: Unknown

Comparable at baseline: Unknown

Causative species: Unknown

Number of people lost to follow-up: $n=692$ participants were randomised, $n=653$ completed both treatment phases of the study, 325/347 participants (93.7\%) in the bifonazole group and 328/345 par- 
EUCTR2008-003215-13 (Continued)

ticipants (95.1\%) in the placebo group. A total of 629 participants completed the whole study period, i.e. until last V5, 311/347 participants (89.6\%) in the bifonazole group and $318 / 345$ participants (92.2\%) in the placebo group

Trial duration: October 23, 2008 - January 21, 2010

Treatment duration: the overall treatment duration per participant was 42 days up to 56 days: 1 st treat-
ment phase (nail ablation) - 14 days (up to 28 days); 2 nd treatment phase (antifungal treatment) 28
days
Drug in study arm 1: bifonazole $1 \%$ cream and urea $40 \%$ paste. Urea: topical application once daily on
infected nail areas (14 days up to 28 days). Bifonazole: topical application once daily on infected nail ar-
eas ( 28 days), $n=347$
Drug in study arm 2: urea $40 \%$ paste and placebo. Urea: topical application once daily on infected nail
areas (14 days up to 28 days); Placebo cream: topical application once daily on infected nail areas ( 28
days), $n=345$

\begin{tabular}{ll}
\hline Outcomes & Complete cure (mycological and clinical cure) at day 14, months 3 and 6 \\
& Mycological cure rate (microscopy and culture) at day 14, months 3 and 6 \\
Adverse events: reported
\end{tabular}

\section{Risk of bias}

\begin{tabular}{|c|c|c|}
\hline Bias & Authors' judgement & Support for judgement \\
\hline \multirow{2}{*}{$\begin{array}{l}\text { Random sequence genera- } \\
\text { tion (selection bias) }\end{array}$} & Unclear risk & \\
\hline & & Comment: Method of randomisation not specified \\
\hline $\begin{array}{l}\text { Allocation concealment } \\
\text { (selection bias) }\end{array}$ & Unclear risk & Comment: No information provided in protocol \\
\hline $\begin{array}{l}\text { Blinding of participants } \\
\text { and personnel (perfor- } \\
\text { mance bias) } \\
\text { All outcomes }\end{array}$ & Unclear risk & Comment: No information provided in protocol \\
\hline $\begin{array}{l}\text { Blinding of outcome as- } \\
\text { sessment (detection bias) } \\
\text { All outcomes }\end{array}$ & Unclear risk & Comment: No information provided in protocol \\
\hline $\begin{array}{l}\text { Incomplete outcome data } \\
\text { (attrition bias) } \\
\text { All outcomes }\end{array}$ & High risk & $\begin{array}{l}\text { Comment: Number of participants discontinuing study provided for each } \\
\text { treatment group, but reasons for discontinuation were not provided }\end{array}$ \\
\hline $\begin{array}{l}\text { Selective reporting (re- } \\
\text { porting bias) }\end{array}$ & Low risk & $\begin{array}{l}\text { Comment: Results to trial registry limited, with data not extractable apart from } \\
\text { mycological cure and safety, but results were reported for all stated outcomes }\end{array}$ \\
\hline Other bias & Low risk & $\begin{array}{l}\text { Comment: Not a multiple intervention trial, all outcomes reported for inter- } \\
\text { ventions }\end{array}$ \\
\hline
\end{tabular}


Methods

RCT (parallel)

Study design: Double blind

Sample size calculation: Unknown

Diagnosis mycology: PCR positive

Big toenail specified: No

Statistical comparisons for outcome: Unknown

Duration of follow-up: 4 weeks

Time points of measurement: At days 28, 46 and weeks $0,4,8,12,16,20,24,36,48$ and 52

Location: Germany (city/institution unknown)

Participants
Age: $18-64$ years old $n=35 ;>65$ years old $n=10$

Sex: Men and women (ratio unknown)

Inclusion criteria: Men and women 18 years old or older, clinical diagnosis of onychomycosis in at least 1 toenail with Onychomycosis Severity Index (OSI) 1 - 15 at screening and baseline (pre-dose D1) and PCR positive at screening, clinical diagnosis of TP with lesions localised to the interdigital spaces or predominantly interdigital, but may extend to other areas of the foot (the non-interdigital lesions should not be hyperkeratotic i.e. should not have the characteristics of tinea pedis moccasin), and confirmed by a positive $\mathrm{KOH}$ wet mount preparation screening and baseline (pre-dose D1), sum of the clinical signs and symptom scores of the target TP lesion is at least 2, including a minimum score of at least 1 for erythema AND a minimum score of 1 for either scaling or pruritus (on a scale of $0-3$, where 1 indicated mild severity), non-menopausal or non-surgically sterilised childbearing potential women must have a negative urine HCG at the time of entry into the study with no intentions of becoming pregnant during the study, non-menopausal or non-surgically sterilised childbearing potential women must use a medically acceptable form of birth control. Participants must have the mental, literate, and legal ability to give written informed consent, which must comply with the International Council for Harmonisation (ICH) guidelines and local requirements. Reliable contraception is defined as a method which results in a low failure rate, i.e. $<1 \%$ per year when used consistently and correctly, such as implants, injectables, some intrauterine contraceptive devices (IUDs), complete sexual abstinence, or a vasectomised partner. Oral contraceptive medications are allowed in this study. Women who are surgically sterile (bilateral tubal ligation, bilateral oophorectomy or hysterectomy) or postmenopausal (defined as no menstrual period within 1 year of screening) are also allowed to participate.

Exclusion criteria: PCR negative OM, OSI $\geq 16, \mathrm{KOH}$ negative tinea pedis, known history of hypersensitivity or allergy to or known contraindication to the use of Lamisil ${ }^{\circledR}$, terbinafine, BB2603 or any of its components/excipients, pregnancy or planning to become pregnant during the study period, breast feeding, severe renal or hepatic impairment with parameters of grade 3 or higher on the corresponding CTCAE scale, presence of other known clinically significant medical and/or psychological illnesses precluding participation, any dermatological condition that could mimic the signs and symptoms of TP or $\mathrm{OM}$, progression of OM by $>50 \%$ nail manifestation during the last 2 months before enrolment, use of any systemic (oral) or topical anti-fungal treatment for onychomycosis in the previous 3 months, use of systemic (oral) antipruritics, including antihistamines, within 72 hours prior to first dosing in the study (Day 1), use of systemic (oral or injectable) corticosteroid or antibiotic therapy within 1 month prior to first dosing in the study (Day 1), use of oral terbinafine or itraconazole (or other oral anti-fungal) within 3 months prior to first dosing in the study, use of topical corticosteroid, antibiotics or antifungal therapy for tinea pedis within 2 weeks prior to first dosing in the study, use of immunosuppressive medication or radiation therapy within 2 months prior to study entry, use of laser therapy, photodynamic therapy, chemical, surgical, relevant mechanical removal for onychomycosis within the last 3 months, confluent, diffuse moccasin-type tinea pedis associated with the entire plantar surface, presence of any other infection of the foot or other disease process that might confound the treatment evaluation, history of dermatophyte infections unresponsive to systemic or topical antifungal drugs (other than onychomycosis), use of any investigational agent within 30 days or 5 half-lives prior to randomisation,

Topical and device-based treatments for fungal infections of the toenails (Review)

Copyright ( 2020 The Cochrane Collaboration. Published by John Wiley \& Sons, Ltd. 
EUCTR2016-001242-25 (Continued)

whichever is longer, screening blood parameters $>2$ times upper limit of normal, unable or unwilling to complete the follow-up evaluations required for the study, any other condition that, in the opinion of the Investigator, would prevent the person from effectively participating in the study, place the person at risk or affect the assessment of efficacy and safety of the study medication, vulnerable people (such as those kept in detention), individuals involved in the planning and/or conduct of the study (i.e. sponsor or employees of sponsor, CRO employees and relatives thereof), any other condition that, in the opinion of the Investigator, would prevent the person from effectively participating in the study, place the person at risk or affect the assessment of efficacy and safety of the study medication, terbinafine resistance should be noted.

Disease duration: Unknown

Comparable at baseline: Unknown

Causative species: Unknown

Number of people lost to follow-up: 1 participant had toe trauma and lost the target toenail

Trial duration: March 28, 2017 - August 13, 2018

Treatment duration: 48 weeks
Drug in study arm 1: BB2603 (terbinafine hydrochloride $0.01 \%), \mathrm{n}=$ unknown
Drug in study arm 2: Lamisil spray $1 \%$ (terbinafine hydrochloride), $\mathrm{n}=$ unknown
Drug in study arm 3: Vehicle spray to BB2603, $\mathrm{n}=$ unknown

Outcomes Complete cure (negative culture and no clinical signs or symptoms, OSI $=0$ ) at weeks $0,4,8,12,16,20$, 24, 36, 48 and 52

Mycological cure (PCR) at week 52

Partial cure (negative culture and $\leq 10 \%$ nail involvement) at weeks $0,4,8,12,16,20,24,36,48$ and 52

Clinical improvement (negative culture and reduction in OSI of at least $40 \%$ from baseline) at weeks 0 , $4,8,12,16,20,24,36,48$ and 52

Adverse events: not reported

Participant-reported outcomes (OnyCOE-t and lifestyle questionnaire)

Pharmacokinetics

Notes Sponsored by industry

\section{Risk of bias}

Bias Authors' judgement Support for judgement

Random sequence genera- Low risk tion (selection bias)

Email response: "A pre-specified randomisation system was used to control allocation of individual eligible subjects...randomisation code was provided by CRO prior to the study."

Comment: Randomisation method likely appropriate

Allocation concealment Low risk
(selection bias)

Email response: "The randomisation scheme and process were developed and (selection bias) managed by the independent CRO pharmacy responsible for study conduct. Sealed envelopes containing the randomisation schedule of the study were opened by the pharmacy for labelling, packaging and randomisation procedures"

Comment: Allocation concealment was appropriate 
EUCTR2016-001242-25 (Continued)

Blinding of participants High risk Email response: "[BB2603 and vehicle] were presented as hand-pump sprays and personnel (perforin indistinguishable PVC bottles. Lamisil spray was open label with the marketed packaging."

mance bias)

All outcomes

Comment: Adequate participant blinding for 2 of 3 treatment groups. Knowledge of the open arm introduces bias

\begin{tabular}{|c|c|c|}
\hline $\begin{array}{l}\text { Blinding of outcome as- } \\
\text { sessment (detection bias) }\end{array}$ & Low risk & $\begin{array}{l}\text { Email response: "Investigators [who assessed clinical outcomes] were blinded } \\
\text { to treatment allocation." }\end{array}$ \\
\hline All outcomes & & Comment: No information provided in protocol \\
\hline
\end{tabular}

Incomplete outcome data Low risk
(attrition bias)

All outcomes

Email response: "A total of 46 subjects were randomised...15 patients met predefined stopping criteria and one patient had toe trauma and lost the target toenail."

Comment: Participants are accounted for and ITT population is identified. Reasons for discontinuation are provided

Selective reporting (re- Low risk Comment: Outcomes provided in clinical trial register are clearly defined
porting bias)

\begin{tabular}{lll}
\hline Other bias $\quad$ Unclear risk $\quad$ Comment: Multiple treatment arm study; but there are no results to assess \\
\hline
\end{tabular}

Gupta 2000a

\begin{tabular}{|c|c|}
\hline \multirow[t]{10}{*}{ Methods } & $\underline{\text { Study } 1}$ \\
\hline & RCT (parallel) \\
\hline & Study design: Double blind \\
\hline & Sample size calculation: No \\
\hline & Diagnosis mycology: $\mathrm{KOH}$ and culture \\
\hline & Big toenail specified: Yes \\
\hline & Statistical comparisons for outcomes: Yes \\
\hline & Duration of follow-up: 12 to 24 weeks \\
\hline & $\begin{array}{l}\text { Time points of measurements: every } 4 \text { weeks of treatment for global evaluations, every } 12 \text { weeks for } \\
\text { mycology and photography planimetry }\end{array}$ \\
\hline & Location: United States (city/institution not stated) \\
\hline \multirow[t]{4}{*}{ Participants } & Total n: 223 \\
\hline & Age: Mean \pm SD (range): ciclopirox $50.4 \pm 12.3(20$ - 70); vehicle $48.6 \pm 13.2(18-70)$ \\
\hline & $\begin{array}{l}\text { Sex: Ciclopirox: men 85/112 (76\%), women 27/112 (24\%). Vehicle: men 90/111 (81\%), women 21/111 } \\
(19 \%)\end{array}$ \\
\hline & $\begin{array}{l}\text { Inclusion criteria: Individuals age } 18 \text { to } 70 \text { years with distal subungual tinea unguium of at least } 1 \text { great } \\
\text { toenail (target nail). Before randomisation, participants were to have clinical evidence of onychomy- } \\
\text { cosis with positive } \mathrm{KOH} \text { preparation and positive dermatophyte culture. Participants had between } \\
20 \% \text { and } 65 \% \text { area of target nail involved (confirmed by computerised planimetry of standardised pho- } \\
\text { tographs) }\end{array}$ \\
\hline
\end{tabular}


Exclusion criteria: Either white superficial or proximal subungual onychomycosis present. People with abnormalities of the target nail that could have prevented obtaining a normal-appearing nail if complete cure of the tinea unguium was achieved. Similarly, individuals with a structural deformity of the target nail or foot that could interfere with photography or planimetric analysis were excluded. An individual with a "spike" of onychomycosis extending to the cuticle of the target nail could not enter the study. A person with a history of immunosuppression or clinical signs indicative of possible immunosuppression was not allowed to enrol in the study

Disease duration: Mean (range) Ciclopirox 11.8 (0.6 - 44) years. Vehicle 11.1 (0.3 - 50) years

Comparable at baseline: Population characteristics were homogeneous between the 2 treatment groups $(P>0.05)$

Causative species: Ciclopirox T rubrum 108/112 (96\%), T. mentagrophytes 4/112 (4\%), E. Floccosum $0 / 112(0 \%)$, other $0 / 112(0 \%)$. Vehicle

Trubrum 108/111 (97\%), T. mentagrophytes 3/111 (3\%), E. Floccosum 0/111 (0\%), other 0/111 (0\%)

Number of people lost to follow-up: Withdrawn (withdrawal of consent, unreliability, violation of protocol criteria, lost to follow-up, and lack of efficacy): treatment $n=23$, vehicle $n=27$

Trial duration: unknown

$\begin{array}{ll}\text { Interventions } & \text { Treatment duration: daily for } 48 \text { weeks } \\ & \text { Drug in study arm 1: Ciclopirox nail lacquer } 8 \%, \mathrm{n}=112 \\ & \text { Drug in study arm 2: Vehicle, } \mathrm{n}=111\end{array}$

Outcomes Treatment success $(\mathrm{KOH}$ and culture and $<10 \%$ area involvement of the target nail plate as determined by planimetry) at week 48

Mycological cure (negative $\mathrm{KOH}$ and culture) at week 48

Treatment cure (KOH and culture and 100\% clear nail) at week 48

Adverse events: reported

\begin{tabular}{ll}
\hline Notes & 3 of 3 authors have ties to industry (e.g. consultant) \\
Quote: "This article is part of a supplement sponsored by [industry]".
\end{tabular}

\section{Risk of bias}

\begin{tabular}{lll}
\hline Bias & Authors' judgement & Support for judgement \\
\hline $\begin{array}{l}\text { Random sequence genera- } \\
\text { tion (selection bias) }\end{array}$ & Unclear risk & $\begin{array}{l}\text { Quote: "randomly assigned" } \\
\text { Comment: Method of randomisation not specified }\end{array}$ \\
\hline $\begin{array}{l}\text { Allocation concealment } \\
\text { (selection bias) }\end{array}$ & Unclear risk & Comment: No information was available \\
\hline $\begin{array}{l}\text { Blinding of participants } \\
\text { and personnel (perfor- } \\
\text { mance bias) } \\
\text { All outcomes }\end{array}$ & Unclear risk & Comment: No information was available \\
\hline $\begin{array}{l}\text { Blinding of outcome as- } \\
\text { sessment (detection bias) } \\
\text { All outcomes }\end{array}$ & Unclear risk & Comment: Incomplete information. Only planimetry is stated as being blinded \\
\hline
\end{tabular}


Gupta 2000a (Continued)

Incomplete outcome data Low risk Comment: Reasons for participants discontinuing the study were provided for (attrition bias) each treatment group. The ITT population was clearly specified

All outcomes
Comment: Review authors were unable to find a clinical trial register entry for this study

Selective reporting (re- Unclear risk porting bias)

Comment: Not a multiple intervention trial; all outcomes reported for interventions

\section{Gupta 2000b}

\section{Methods}

\section{Study 2}

RCT (parallel)

Study design: Double blind

Sample size calculation: No

Diagnosis mycology: $\mathrm{KOH}$ and culture

Big toenail specified: Yes

Statistical comparisons for outcomes: Yes

Duration of follow-up: 12 to 24 weeks

Time points of measurements: every 4 weeks of treatment for global evaluations, every 12 weeks for mycology and photography planimetry

Location: United States (city/institution unknown)

Participants

Total n: 237

Age: Mean \pm SD (range): ciclopirox $49.6 \pm 11.9(19-70)$, vehicle $50.1 \pm 12.2(23-70)$

Sex: Ciclopirox: men 94/119 (79\%), women 25/119 (21\%). Vehicle: men 89/118 (75\%), women 29/118 $(25 \%)$

Inclusion criteria: Individuals age 18 to 70 years with distal subungual tinea unguium of at least 1 great toenail (target nail). Before randomisation, patients were to have clinical evidence of onychomycosis with positive $\mathrm{KOH}$ preparation and positive dermatophyte culture. Participants had between $20 \%$ to $65 \%$ area of target nail involved (confirmed by computerised planimetry of standardised photographs)

Exclusion criteria: If either white superficial or proximal subungual onychomycosis was present. Also excluded were people with abnormalities of the target nail that could have prevented obtaining a normal-appearing nail if complete cure of the tinea unguium was achieved. Similarly, individuals with a structural deformity of the target nail or foot that could interfere with photography or planimetric analysis were excluded. An individual with a "spike" of onychomycosis extending to the cuticle of the target nail could not enter the study. A person with a history of immunosuppression or clinical signs indicative of possible immunosuppression was not allowed to enrol in the study

Disease duration: Mean (range) ciclopirox 10.8 (0.5 - 51) years, vehicle $11.6(0.5-50)$ years

Comparable at baseline: Population characteristics were homogeneous between the 2 treatment groups $(P>.05)$

Causative species: Ciclopirox T rubrum 114/119 (96\%), T. mentagrophytes 5/119 (4\%), E. fluccosum 0/119 (0\%), other 0/119 (0\%). Vehicle 
Number of people lost to follow-up: Withdrawn (withdrawal of consent, unreliability, violation of protocol criteria, lost to follow-up, and lack of efficacy): treatment $n=23$, vehicle $n=24$

Trial duration: unknown

\begin{tabular}{ll}
\hline Interventions & Treatment duration: daily for 48 weeks \\
& Drug in study arm 1: Ciclopirox nail lacquer $8 \%, \mathrm{n}=119$ \\
& Drug in study arm 2: Vehicle, $\mathrm{n}=118$ \\
\hline Outcomes & $\begin{array}{l}\text { Treatment success (KOH and culture and }<10 \% \text { area involvement of the target nail plate as determined } \\
\text { by planimetry) at week } 48\end{array}$ \\
Mycological cure (negative KOH and culture) at week 48 \\
Treatment cure (KOH and culture and $100 \%$ clear nail) at week 48 \\
Adverse events: reported \\
\hline Notes 3 authors have ties to industry (e.g. consultant) \\
Quote: "This article is part of a supplement sponsored by [industry]"
\end{tabular}

\section{Risk of bias}

\begin{tabular}{|c|c|c|}
\hline Bias & Authors' judgement & Support for judgement \\
\hline $\begin{array}{l}\text { Random sequence genera- } \\
\text { tion (selection bias) }\end{array}$ & Unclear risk & $\begin{array}{l}\text { Quote: "randomly assigned" } \\
\text { Comment: Method of randomisation not specified }\end{array}$ \\
\hline $\begin{array}{l}\text { Allocation concealment } \\
\text { (selection bias) }\end{array}$ & Unclear risk & Comment: No information was available \\
\hline $\begin{array}{l}\text { Blinding of participants } \\
\text { and personnel (perfor- } \\
\text { mance bias) } \\
\text { All outcomes }\end{array}$ & Unclear risk & Comment: No information was available \\
\hline $\begin{array}{l}\text { Blinding of outcome as- } \\
\text { sessment (detection bias) } \\
\text { All outcomes }\end{array}$ & Unclear risk & Comment: Incomplete information. Only planimetry is stated as being blinded \\
\hline $\begin{array}{l}\text { Incomplete outcome data } \\
\text { (attrition bias) } \\
\text { All outcomes }\end{array}$ & Low risk & $\begin{array}{l}\text { Comment: Reasons for patients discontinuing the study were provided for } \\
\text { each treatment group. The ITT population was clearly specified }\end{array}$ \\
\hline $\begin{array}{l}\text { Selective reporting (re- } \\
\text { porting bias) }\end{array}$ & Unclear risk & $\begin{array}{l}\text { Comment: Review authors were unable to find a clinical trial register entry for } \\
\text { this study }\end{array}$ \\
\hline Other bias & Low risk & $\begin{array}{l}\text { Comment: Not a multiple intervention trial, all outcomes reported for inter- } \\
\text { ventions }\end{array}$ \\
\hline
\end{tabular}

Methods RCT (parallel)


Gupta 2006 (Continued)

\author{
Study design: Not blinded \\ Sample size calculation: No \\ Diagnosis mycology: Not specified \\ Big toenail specified: Yes \\ Statistical comparison for outcomes: No \\ Duration of follow-up: At 24 weeks \\ Time points of measurements: Weeks 12 and 24 \\ Location: unknown
}

Total n: 120
Age: unknown
Sex: unknown
Inclusion criteria: Chinese adults with dermatophyte onychomycosis of at least 1 great toenail
Exclusion criteria: Unknown
Disease duration: Unknown
Comparable at baseline: Unknown
Causative species: Dermatophytes
Number of people lost to follow-up: 0
Trial duration: unknown

Interventions

Treatment duration: daily for 6 or 12 weeks

Placebo lacquer-12 wks, $n=12$

$1 \%$ terbinafine nail lacquer (TNL)- $6 \mathrm{wks}, \mathrm{n}=12$

$1 \%$ TNL- 12 weeks, $\mathrm{n}=20$

$5 \%$ TNL- 6 weeks, $n=12$

$5 \%$ TNL-12 weeks, $\mathrm{n}=20$

$10 \%$ TNL-6 weeks, $\mathrm{n}=12$

$10 \%$ TNL-12 weeks, $n=20$

Ciclopirox 8\% nail lacquer (CNL)-12 weeks, $\mathrm{n}=12$

Outcomes Treatment success ( $\mathrm{KOH}$ and culture with a new unaffected length $\geq 3 \mathrm{~mm}$ at week 12 ) at weeks 12 and 24

Mycological success (KOH and culture) at weeks 12 and 24

Adverse events: reported
Notes
Conference abstract. Author has ties to industry (e.g. investigator, past honoraria and grants). Study partly funded by industry

\title{
Risk of bias
}

Topical and device-based treatments for fungal infections of the toenails (Review) 
Gupta 2006 (Continued)

\begin{tabular}{lll} 
Bias & Authors' judgement & Support for judgement \\
\hline $\begin{array}{l}\text { Random sequence genera- } \\
\text { tion (selection bias) }\end{array}$ & Unclear risk & $\begin{array}{l}\text { Quote: "randomised" } \\
\text { Comment: Method of randomisation not spe }\end{array}$ \\
\hline $\begin{array}{l}\text { Allocation concealment } \\
\text { (selection bias) }\end{array}$ & Unclear risk & Comment: No information was available \\
\hline $\begin{array}{l}\text { Blinding of participants } \\
\text { and personnel (perfor- } \\
\text { mance bias) }\end{array}$ & Unclear risk & Comment: No information was available \\
All outcomes & & \\
\hline
\end{tabular}

\begin{tabular}{lll}
\hline $\begin{array}{l}\text { Blinding of outcome as- } \\
\text { sessment (detection bias) } \\
\text { All outcomes }\end{array}$ & Unclear risk & Comment: No information was available \\
\hline $\begin{array}{l}\text { Incomplete outcome data } \\
\text { (attrition bias) } \\
\text { All outcomes }\end{array}$ & Unclear risk & Comment: No information was available \\
\hline $\begin{array}{l}\text { Selective reporting (re- } \\
\text { porting bias) }\end{array}$ & Unclear risk & $\begin{array}{l}\text { Comment: Review authors were unable to find a clinical trial register entry for } \\
\text { this study }\end{array}$ \\
\hline Other bias & Low risk & $\begin{array}{l}\text { Comment: Multiple intervention study, but outcomes for all interventions re- } \\
\text { ported separately }\end{array}$ \\
\hline
\end{tabular}

RCT (parallel)
Study design: Not blinded
Sample size calculation: Unknown
Diagnosis mycology: KOH and culture
Big toenail specified: Yes
Statistical comparison for outcomes: Yes
Duration of follow up: 24 weeks
Time points of measurements: Weeks 12, 24 and 48
Location: unknown
$\begin{aligned} & \text { Total n: } 60 \\ & \text { Age: Unknown } \\ & \text { Sex: Unknown } \\ & \text { Inclusion criteria: Men and women aged } 12-80 \text { years of age with mild-to-moderate distal subungual } \\ & \text { onychomycosis due to dermatophytes (confirmed by KOH and culture), having } 25 \% \text { to } 60 \% \text { diseased } \\ & \text { big toenail area }\end{aligned}$


Exclusion criteria: People with history of cardiovascular, renal, neurologic, liver, immunologic or endocrine dysfunction if they are clinically significant. People with a recent history ( $<1$ year) of myocardial infarction and/or ( $<3$ years) of heart failure or those with any cardiac arrhythmia requiring drug therapy. People with liver function impairment AST, ALT more than twice above the upper limit of reference range. People with kidney function impairment serum creatinine more than twice above the upper limit of reference range. History of cancer within the past 5 years, excluding treated basal cell carcinoma. Chemotherapy, immunosuppressive therapy in the 12 weeks prior to screening visit. Systemic glucocorticosteroids, antimetabolites and immunostimulants therapy in the 4 weeks prior to screening visit, alcohol or substance abuse, HIV infection or any other immunodeficiency, history of allergic reactions to terbinafine or its excipients, use of an investigational drug or participation in an investigational study within 30 days prior to administration of study medication. Patients using nail polish or other nail cosmetic product on the concerned nails, target nail hyperkeratosis exceeding $4 \mathrm{~mm}$ in thickness, proximal subungual involvement, mucocutaneous candidiasis, white superficial onychomycosis, onychomycosis caused by yeasts or non-dermatophytes mould, "Yellow spikes" on the target nail, lunula involvement, severe plantar or moccasin tinea pedis, nail abnormalities due to conditions like psoriasis or lichen planus, uncontrolled diabetes, recurrent erysipelas at screening visit. Use of systemic antifungal drugs in the 6 months prior to screening visit, use of topical products containing terbinafine in the 3 months prior to screening visit, use of topical antifungal drugs (other than terbinafine) in the 4 weeks prior to screening visit, breast-feeding women, positive urine pregnancy test at screening (performed on all women of child-bearing potential or being in non-surgical post-menopause for less than 1 year), women of child-bearing potential or in menopause for less than 1 year not using a reliable method of contraception

Disease duration: Unknown

Comparable at baseline: Unknown

Causative species: Dermatophyte

Number of people lost to follow-up: 3 participants did not complete the study; 2 in $5 \%$ group and 1 in the $10 \%$ group

Trial duration: unknown

Drug in study arm 3: P-3058 10\% weekly for 24 weeks, $n=20$

\begin{tabular}{ll} 
Outcomes & Cure (culture and $\mathrm{KOH}$ ) at weeks $12,24,48$ \\
& Adverse events: reported \\
\hline Notes & $\begin{array}{l}\text { Conference abstract. } 3 \text { of six authors are employees of industry. The study was } 100 \% \text { sponsored by in- } \\
\text { dustry }\end{array}$
\end{tabular}

\section{Risk of bias}

\begin{tabular}{lll}
\hline Bias & Authors' judgement & Support for judgement \\
\hline $\begin{array}{l}\text { Random sequence genera- } \\
\text { tion (selection bias) }\end{array}$ & Unclear risk & $\begin{array}{l}\text { Quote: "randomly allocated" } \\
\text { Comment: Method of randomisation not specified }\end{array}$ \\
\hline $\begin{array}{l}\text { Allocation concealment } \\
\text { (selection bias) }\end{array}$ & Unclear risk & Comment: No information was available \\
\hline $\begin{array}{l}\text { Blinding of participants } \\
\begin{array}{l}\text { and personnel (perfor- } \\
\text { mance bias) }\end{array}\end{array}$ & High risk & Quote: "open label" \\
\end{tabular}


Hartmane 2013 (Continued)

All outcomes

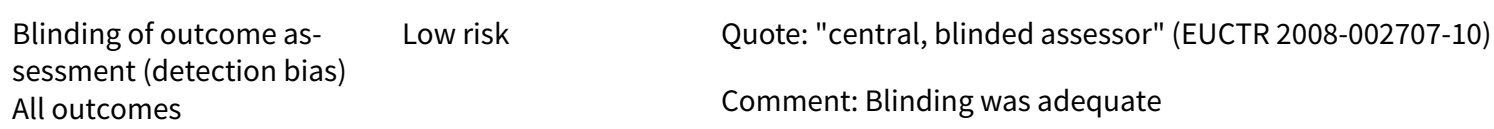

All outcomes

Comment: Attrition rates provided, but reasons for dropout not provided. The

Incomplete outcome data Low risk

ITT was clearly specified

All outcomes

Selective reporting (re- High risk
porting bias)

Comment: Primary endpoint identified in protocol not reported in the article. porting bias) EUCTR 2008-002707-10

Other bias High risk

Hollmig 2014

Rethods (parallel)
Study design: Not blinded
Sample size calculation: Yes
Diagnosis mycology: PAS stain and culture
Big toenail specified: No
Statistical comparisons for outcomes: Yes
Duration of follow-up: At 3 and 12 months
Time points of measurements: Months 3 and 12
Location: Stanford University, Stanford, California, United States

Participants

Total n: 27

Age: Mean (range): treatment 53 years, control 65 years, $(18-75)$

Sex: Treatment: men 10/12 (83\%), women 2/12 (16.7\%). Control: men 8/10 (80\%), women 2/10 (20\%)

Inclusion criteria: Diagnosis of onychomycosis by clinical toenail morphology confirmed by positive culture. People whose cultures revealed nondermatophyte molds were included if periodic acid Schiff staining-assisted microscopic evaluation was positive ( 4 in laser group; 3 in control group). All participants met diagnostic criteria for onychomycosis as defined by prior studies. Participants were not excluded based on the severity of disease or prior treatment regimen

Exclusion criteria: Unknown

Disease duration: Unknown

Comparable at baseline: Participants in the laser and control groups differed in age (64.9 vs 53.4 years, $\mathrm{P}=0.03$ ) but not in gender, interval days for follow-up, extent of clinical nail involvement, or per cent with dermatophyte species at baseline culture

Causative species: dermatophyte and non-dermatophyte moulds

Number of people lost to follow-up: 5 in laser group lost at 3-month follow-up, did not return for reevaluation after treatment 
Hollmig 2014 (Continued)

Patient enrolment: July 2011 - December 2011
Treatment duration: Participants in the laser group underwent 2 treatments separated by 2 weeks

Treatment in study arm 1: $1064 \mathrm{~nm}$ laser, $\mathrm{n}=17$

Treatment in study arm 2: control (not treated), $n=10$

\begin{tabular}{ll}
\hline Outcomes & Mycological cure (culture) at months 3 and 12 \\
& Proximal nail plate clearance in mm at months 3 and 12 \\
& Adverse events: reported \\
\hline Notes & $\begin{array}{l}\text { All } 6 \text { authors have no conflicts of interest to declare. The laser handpiece was loaned to the University } \\
\text { department by industry for the study }\end{array}$ \\
\hline
\end{tabular}

\section{Risk of bias}

\begin{tabular}{ll}
\hline Bias & Authors' jud \\
\hline $\begin{array}{l}\text { Random sequence genera- } \\
\text { tion (selection bias) }\end{array}$ & Low risk
\end{tabular}

Quote: "Patients were randomised following simple randomisation procedures (computerized random number generator) in a 2:1 ratio into laser or control groups."

Comment: Specific randomisation method that is suitable was specified

\begin{tabular}{ll}
\hline $\begin{array}{l}\text { Allocation concealment } \\
\text { (selection bias) }\end{array}$ & Low risk \\
& $\begin{array}{l}\text { Email response: Randomisation was performed by research staff, not study in- } \\
\text { vestigators. }\end{array}$ \\
Comment: Allocation concealment was likely appropriate
\end{tabular}

Blinding of participants High risk Comment: The study was not blinded
and personnel (perfor-
mance bias)
All outcomes

\begin{tabular}{|c|c|c|}
\hline $\begin{array}{l}\text { Blinding of outcome as- } \\
\text { sessment (detection bias) } \\
\text { All outcomes }\end{array}$ & High risk & Comment: The study was not blinded \\
\hline $\begin{array}{l}\text { Incomplete outcome data } \\
\text { (attrition bias) } \\
\text { All outcomes }\end{array}$ & Low risk & $\begin{array}{l}\text { Comment: Reasons for patients discontinuing the study were provided for } \\
\text { each treatment group. The ITT population was clearly specified }\end{array}$ \\
\hline $\begin{array}{l}\text { Selective reporting (re- } \\
\text { porting bias) }\end{array}$ & Low risk & $\begin{array}{l}\text { Comment: Outcomes reported in the publication are the same as those in pro- } \\
\text { tocol. NCT01666002 }\end{array}$ \\
\hline Other bias & Low risk & $\begin{array}{l}\text { Comment: Not a multiple intervention trial, all outcomes reported for inter- } \\
\text { ventions }\end{array}$ \\
\hline
\end{tabular}

Ijzerman 2010

$\begin{array}{ll}\text { Methods } & \text { RCT (parallel) } \\ & \text { Study design: Double blind } \\ & \text { Sample size calculation: Unknown }\end{array}$

Topical and device-based treatments for fungal infections of the toenails (Review)

Copyright $\odot 2020$ The Cochrane Collaboration. Published by John Wiley \& Sons, Ltd. 
ljzerman 2010 (Continued)

Diagnosis mycology: $\mathrm{KOH}$ and culture

Big toenail specified: Yes

Statistical comparisons for outcomes: Yes

Duration of follow-up: 4 and 8 weeks following treatment

Time points of measurements: Weeks 6, 12, 24, 32, 46, 50

Location: United States (city/institution unknown)

\section{Participants}

Total $n=443$ (safety population), intent-to-treat (ITT) or per-protocol (PP) populations unknown

Age: 18 - 75 years of age, mean 52 years

Sex: men $n=372 ;$ women $n=71$

Inclusion criteria: Men and women between the ages of 18 and 75 with $\mathrm{KOH}$ - and culture-confirmed distal subungual onychomycosis of at least 1 great toe, involving $25 \%$ to $67 \%$ of the great toenail, without lunular or proximal involvement. Culture-confirmed for a dermatophyte. Must refrain from using any lotions, creams, liquids, or polish on treated toenails or on the skin immediately adjacent to the toenails during the treatment period unless directed to do so by the investigator. Willing to refrain from using topical steroids or topical antifungals on toenails or the skin immediately adjacent to the toenails; or systemic antifungals for the duration of the study

Exclusion criteria: Women who are pregnant, plan to become pregnant during the study, or are nursing a child; are hypersensitive to topical creams, ointments, medications, or surfactants; have received systemic antifungal therapy for any reason within 3 months, or topical antifungal therapy on the toenails or skin immediately adjacent to the toenails within 3 weeks prior to the start of the study; or have taken any investigational drug within 4 weeks prior to the start of the study

Disease duration: Unknown

Comparable at baseline: Uknown

Causative species: Unknown

Number of people lost to follow-up: Unknown

Trial duration: unknown

Drug in study arm 1: NB-002 oil in water emulsion, $0.25 \%$ twice daily

Drug in study arm 2: NB-002 oil in water emulsion, $0.5 \%$ once daily

Drug in study arm 3: NB-002 oil in water emulsion, 0.5\% twice daily

Drug in study arm 4: Vehicle once or twice daily

Complete cure at week 50
Therapeutic success at week 50
Mycologic cure at week 50
Adverse events: reported

Notes Conference abstract. Industry provided support

\section{Risk of bias}


Ijzerman 2010 (Continued)

\begin{tabular}{lll}
$\begin{array}{l}\text { Random sequence genera- } \\
\text { tion (selection bias) }\end{array}$ & Unclear risk & $\begin{array}{l}\text { Quote: "randomised" } \\
\text { Comment: Method of randomisation not specified }\end{array}$ \\
\hline $\begin{array}{l}\text { Allocation concealment } \\
\text { (selection bias) }\end{array}$ & Unclear risk & Comment: No information was available \\
\hline $\begin{array}{l}\text { Blinding of participants } \\
\begin{array}{l}\text { and personnel (perfor- } \\
\text { mance bias) } \\
\text { All outcomes }\end{array}\end{array}$ & Unclear risk & Comment: No information was available \\
\hline
\end{tabular}

\begin{tabular}{lll}
\hline $\begin{array}{l}\text { Blinding of outcome as- } \\
\text { sessment (detection bias) } \\
\text { All outcomes }\end{array}$ & Unclear risk & Comment: No information was available \\
\hline $\begin{array}{l}\text { Incomplete outcome data } \\
\text { (attrition bias) } \\
\text { All outcomes }\end{array}$ & Unclear risk & Comment: No information was available \\
\hline $\begin{array}{l}\text { Selective reporting (re- } \\
\text { porting bias) }\end{array}$ & Low risk & $\begin{array}{l}\text { Comment: Outcomes reported in conference abstract are included in protocol. } \\
\text { NCT00453271 }\end{array}$ \\
\hline Other bias & Unclear risk & $\begin{array}{l}\text { Comment: Multiple intervention trial. Unclear from conference abstract if out- } \\
\text { comes were reported for each arm separately }\end{array}$ \\
\hline
\end{tabular}

Iorizzo 2016

\begin{tabular}{ll}
\hline Methods & RCT (parallel) \\
Study design: Open (blinded evaluator) \\
Sample size calculation: Yes \\
Diagnosis mycology: $\mathrm{KOH}$ and culture \\
Big toenail specified: Yes \\
Statistical comparisons for outcomes: Yes \\
Duration of follow-up: Unknown \\
Time points of measurements: Weeks 4, 8, 12, 24, and 48 \\
Location: Russian Federation and Latvia (cities/institutions not stated)
\end{tabular}

Participants

Total n: 120

Age: Mean \pm SD: P-3051 $51.45 \pm 11.75$, amorolfine $53.85 \pm 12.21(18-75)$ years.

Sex: P-3051: men 6/60 (10.0\%), women 54/60 (90\%). Amororlfine: men 10/60 (16.7\%), women 50/60 $(83.3 \%)$

Inclusion criteria: Patients with mild-to-moderate toenail distal lateral subungual onychomycosis, caused by dermatophytes, yeasts and moulds, of at least 1 big toenail (target nail), without the presence of yellow spikes, dermatophytoma or lunula involvement. According to the protocol, participants, $18-75$ years old, had to sign an informed consent form before enrolment. Those participants with an infected target nail area $\geq 25$ and $\leq 75 \%$, and with both positive $\mathrm{KOH}$ and culture for fungal nail pathogens at screening, were enrolled in the trial 
Iorizzo 2016 (Continued)

Exclusion criteria: Concomitant severe plantar tinea pedis, other nail abnormalities (such as psoriasis or lichen planus) and the use of any systemic or topical treatment

Disease duration: Unknown

Comparable at baseline: Both groups were homogeneous with respect to sex, age and weight, as well as to the number of affected toenails, causative pathogens and the percentage of the infected target nail area

Causative species: P-3051: Dermatophytes $43 / 60$ (71.7\%), yeast 12/60 (20.0\%), nondermatophyte moulds 5/60 (8.3\%). Amorolfine: dermatophytes $47 / 60$ (78.3\%), yeast $11 / 60$ (18.3\%), nondermatophyte moulds $2 / 60(3.4 \%)$

Number of people lost to follow-up: Unknown

Participant enrolment: Feb 21, 2012 - May 27, 2014

Interventions

Drug in study arm 1: P-3051 (ciclopirox 8\%) daily for 48 weeks, $n=60$

Drug in study arm 2: Amorolfine 5\% twice weekly for 48 weeks, $n=60$

Mycological cure (culture and $\mathrm{KOH}$ )
Complete cure (negative $\mathrm{KOH}$ microscopy and negative culture with no residual clinical involvement of
the target toenail) at weeks $4,8,12,24$, and 48
Treatment success (negative $\mathrm{KOH}$ microscopy and negative culture as well as nor residual involvement
of the target toenail) at weeks $4,8,12,24$, and 48

Adverse events: reported

All 4 authors have no conflicts of interest. The study was funded by industry

\title{
Risk of bias
}

\section{Bias} Authors' judgement Support for judgement

Random sequence genera- Low risk tion (selection bias)

\begin{abstract}
Email response: "Eligible patients have been randomised to one of the two treatment groups according to a permutated blocks random codes generated by the study statistician through a computer program allocating the patient in a progressive temporal sequence to the treatment random number assigned by the system."

Comment: Specific randomisation method that is suitable was specified
\end{abstract}

\begin{tabular}{ll}
\hline $\begin{array}{l}\text { Allocation concealment } \\
\text { (selection bias) }\end{array}$ & Low risk \\
& $\begin{array}{l}\text { Quote: "The investigator allocated the treatments...to each patient according } \\
\text { to a randomisation list." }\end{array}$ \\
& Comment: Allocation concealment was appropriate \\
\hline
\end{tabular}

Blinding of participants High risk Comment: Participants and personnel were not blinded
and personnel (perfor-
mance bias)
All outcomes

\begin{tabular}{lll}
$\begin{array}{l}\text { Blinding of outcome as- } \\
\text { sessment (detection bias) } \\
\text { All outcomes }\end{array}$ & Low risk & $\begin{array}{l}\text { Comment: A blinded evaluator was used for efficacy outcomes; but safety was } \\
\text { recorded by unblinded investigators }\end{array}$ \\
\hline $\begin{array}{l}\text { Incomplete outcome data } \\
\text { (attrition bias) }\end{array}$ & Low risk & $\begin{array}{l}\text { Email response: "All patients completed the study, none were lost to follow } \\
\text { All outcomes }\end{array}$
\end{tabular}


Iorizzo 2016 (Continued)

Comment: The ITT population was clearly specified

\begin{tabular}{|c|c|c|}
\hline $\begin{array}{l}\text { Selective reporting (re- } \\
\text { porting bias) }\end{array}$ & High risk & $\begin{array}{l}\text { Comment: Outcomes reported in the publication are different from those in } \\
\text { protocol. EUCTR2011-003087-70 }\end{array}$ \\
\hline
\end{tabular}

Other bias Low risk Comment: Not a multiple intervention trial, but all outcomes reported for in-
terventions

\section{Karsai 2017}

\begin{tabular}{ll}
\hline Methods & RCT (parallel) \\
Study design: Single blind (investigator blind) \\
Sample size calculation: No \\
Diagnosis mycology: PAS stain and culture \\
Big toenail specified: No \\
Statistical comparisons for outcomes: Yes \\
Duration of follow-up: At 12 months \\
Time points of measurements: Month 12 \\
Location: Medizinisches Versorgungszentrum Dres. Raulin and Kollegen, Karlsruhe, Germany
\end{tabular}

Participants

Total n: 22

Age: Mean \pm SD (range): treatment $69.4 \pm 12$, control $65.9 \pm 17$ years, $(18<)$

Sex: Treatment: men 7/10 (70\%), women 3/10 930\%). Control: male 9/10 (90\%), female 1/10 (10\%)

Inclusion criteria: Pople over the age of 18 with a clinical diagnosis of distolateral subungual onychomycosis were enrolled consecutively; the diagnosis was confirmed by means of positive dermatophyte culture on a selective agar plate after incubation at $30^{\circ} \mathrm{C}$ for 4 weeks. If the fungal culture was negative, a histopathological examination was conducted at an external laboratory using PAS stain on representative nail clippings

Exclusion criteria: Nails with a negative PAS stain were not included. Other exclusion criteria were as follows: other diseases of the nails (e.g. nail psoriasis); a history of previous trauma to the target nail; discolouration of the nail (other than 'yellow streaks'); a history of peripheral arterial disease or diabetes mellitus; peripheral neuropathy of any kind; allergy to amorolfine or any other ingredient in Loceryl cream; application of topical antimycotics (within the 3 months prior to study enrolment); systemic use of antimycotics (within the 12 months prior to study enrolment); pregnancy or lactation

Disease duration: Unknown

Comparable at baseline: A comparison of both groups' OSI scores showed no significant differences at baseline $(P=0.9873)$

Causative species: In both groups, toenails had solely a Trichophyton rubrum infection

Number of participants lost to follow-up: Laser: withdrew by follow-up (12 months) or stopped treatment $\mathrm{n}=0$. Control: withdrew by follow-up (12 months) $\mathrm{n}=2$; did not return and/or not reachable

Trial duration: February 2013 - February 2015

Interventions

Treatment duration: 4 treatments at intervals of $4-6$ weeks with amorolfine prophylaxis for skin of feet and toes 
Treatment in study arm 2: Control (not treated), $n=12$

\begin{tabular}{ll}
\hline Outcomes & Mycological cure (culture and PAS) at month 12 \\
& Clinical appearance using Onychomycosis severity index (OSI) at month 12 \\
& Adverse events: reported \\
\hline Notes & $\begin{array}{l}\text { Quote: "There is no conflict of interest. None of the authors of the study has financial interests of any } \\
\text { kind or is in any way affiliated with manufacturers, wholesalers or retailers of the device under investi- } \\
\text { gation. Funding sources: None" }\end{array}$ \\
\hline
\end{tabular}

\section{Risk of bias}

\section{Bias}

\section{Authors' judgement Support for judgement}

Random sequence genera- Low risk tion (selection bias)

Quote: "An independent medical assistant randomised the patients to the treatment group or the control group. The randomisation list was generated using a web based program, and the randomised allocation took place by assigning even numbers to the treatment group and odd numbers to the control group"

Comment: Specific randomisation method that is suitable was specified

\begin{tabular}{|c|c|c|}
\hline $\begin{array}{l}\text { Allocation concealment } \\
\text { (selection bias) }\end{array}$ & Low risk & $\begin{array}{l}\text { Quote: "An independent medical assistant randomised the patients to the } \\
\text { treatment group or the control group." }\end{array}$ \\
\hline
\end{tabular}

Comment: Allocation concealment was appropriate

Blinding of participants High risk Comment: Participants and personnel were not blinded to treatment
and personnel (perfor-
mance bias)

\begin{tabular}{|c|c|c|}
\hline $\begin{array}{l}\text { Blinding of outcome as- } \\
\text { sessment (detection bias) }\end{array}$ & Low risk & $\begin{array}{l}\text { Quote: "The assessment was performed by two independent blinded investi- } \\
\text { gators who were not otherwise involved in the study" }\end{array}$ \\
\hline All outcomes & & Comment: Blinding was appropriate \\
\hline
\end{tabular}

\begin{tabular}{lll}
\hline $\begin{array}{l}\text { Incomplete outcome data } \\
\text { (attrition bias) } \\
\text { All outcomes }\end{array}$ & Low risk & $\begin{array}{l}\text { Comment: Reasons for patients discontinuing the study were provided for } \\
\text { each treatment group. The ITT population was clearly specified }\end{array}$ \\
\hline $\begin{array}{l}\text { Selective reporting (re- } \\
\text { porting bias) }\end{array}$ & High risk & $\begin{array}{l}\text { Comment: Outcomes reported in the publication are different from those in } \\
\text { protocol. DRKS00007709 }\end{array}$ \\
\hline Other bias & Low risk & $\begin{array}{l}\text { Comment: Not a multiple intervention trial, all outcomes reported for inter- } \\
\text { ventions }\end{array}$ \\
\hline
\end{tabular}

Koren 2018

$\begin{array}{ll}\text { Methods } & \text { RCT (parallel) } \\ & \text { Study design: Open } \\ & \text { Sample size calculation: No }\end{array}$

Topical and device-based treatments for fungal infections of the toenails (Review)

Copyright $\odot 2020$ The Cochrane Collaboration. Published by John Wiley \& Sons, Ltd. 
Koren 2018 (Continued)

Diagnosis mycology: Culture

Big toenail specified: Yes

Statistical comparisons for outcomes: Yes

Duration of follow-up: At 3 and 9 months

Time points of measurements: Months 3 and 9

Location: Tel Aviv Medical Center, Tel Aviv, Israel

Total $\mathrm{n}: 60$
Age: Mean 49 (Range 26 - 66)
Sex: Men and women (ratio unknown)
Inclusion criteria: People 18 - 60 years of age with a clinical diagnosis of bilateral toenail onychomyco-
Sis and positive mycological culture
Exclusion criteria: Systemic antifungal treatment during the past 12 months, concomitant nail disease,
vascular or microcirculatory disorders, metabolic diseases, immunodeficiency, paresis and paralysis
from CNS or PNS injury, pregnancy, and porphyria, photosensitive dermatoses, and allergy to photo-
sensitisers
Disease duration: Mean 11.7 years
Comparable at baseline: Unknown
Causative species: $94 \%$ T. rubrum
Number of people lost to follow-up: Amorolfine group $n=4$ due to lack of compliance
Trial duration: unknown

Interventions Treatment duration: 6 treatment sessions at 3-week intervals with amorolfine applied once weekly for 9 months

Intervention in study arm 1: Fractional ablative CO2 laser followed by 20\% ALA-photodynamic therapy, $\mathrm{n}=30$

Intervention in study arm 2: Fractional ablative $\mathrm{CO} 2$ laser followed by amorolfine $5 \%$ lacquer, $\mathrm{n}=30$

Outcomes Clinical improvement at months 3 and 9, measured on a $0-5$ scale with $0=$ no response to treatment and 5 = fully normal-appearing nail

Mycological culture at months 3 and 9

Patient satisfaction

Adverse events: reported

Notes Q Quote: "The authors have no conflicts of interest to declare."

\section{Risk of bias}

\begin{tabular}{lll}
\hline Bias & Authors' judgement & Support for judgement \\
\hline $\begin{array}{l}\text { Random sequence genera- } \\
\text { tion (selection bias) }\end{array}$ & Low risk & $\begin{array}{l}\text { Email response: "randomly assigned to } 2 \text { study groups. An excel table was pre- } \\
\text { pared in advance and patients were added according to the order of refer- } \\
\text { ral/admission to the study. The randomisation occurred before the first study } \\
\text { visit in which clinical and demographic data were obtained." }\end{array}$
\end{tabular}




Allocation concealment High risk
(selection bias)

Email response: "The investigators who provided the treatment were aware of the randomisation for the two study groups."

Comment: Allocation concealment was not appropriate

\begin{tabular}{|c|c|c|}
\hline $\begin{array}{l}\text { Blinding of participants } \\
\text { and personnel (perfor- } \\
\text { mance bias) }\end{array}$ & High risk & $\begin{array}{l}\text { Quote: "open-label" } \\
\text { Comment: There was no blinding }\end{array}$ \\
\hline
\end{tabular}

Blinding of outcome as- Low risk sessment (detection bias)

All outcomes
Quote: "photographs were compared by 2 dermatologists...the dermatologists were blinded to the study groups"

Email response: "The clinical picture evaluators and the laboratory mycological evaluators were blinded to the study groups."

Comment: Blinding was adequate

\begin{tabular}{lll}
\hline $\begin{array}{l}\text { Incomplete outcome data } \\
\text { (attrition bias) } \\
\text { All outcomes }\end{array}$ & Low risk & $\begin{array}{l}\text { Comment: Reasons for participants discontinuing the study were provided for } \\
\text { each treatment group. The ITT population was clearly specified }\end{array}$ \\
\hline $\begin{array}{l}\text { Selective reporting (re- } \\
\text { porting bias) }\end{array}$ & Low risk & $\begin{array}{l}\text { Email response: "The trial was not registered." } \\
\text { Comment: Outcomes specified in method reported in results }\end{array}$ \\
\hline $\begin{array}{l}\text { Other bias } \\
\text { Low risk }\end{array}$ & $\begin{array}{l}\text { Comment: Not a multiple intervention trial, all outcomes reported for inter- } \\
\text { ventions }\end{array}$
\end{tabular}

Kumar 2001

Rethods (parallel)
Study design: Double blind
Sample size calculation: No
Diagnosis mycology: $\mathrm{KOH}$ and culture
Big toenail specified: No
Statistical comparisons for outcomes: No
Duration of follow-up: At 2 weeks
Time points of measurements: Weeks 2, 4, 6, 8, 10 and 12
Location: Bangalore (city/institution unknown)
Total n: 43
Age: Mean (range): 49 years (21 - 70)
Sex: Men $64 \%$, women $36 \%$
Inclusion criteria: Clinical diagnosis of distal subungual or proximal onychomycosis or the presence
of dermatophytes in mycological culture. All participants withdraw their systemic antifungal treat-
ment and topical treatment 1 month prior to taking samples for baseline mycological culture. Partici-


Kumar 2001 (Continued)

pants were screened before starting the trial treatment and those with clinically suspected toenail infection were included. To exclude any possibility of previous treatment with antimycotic agents, it was mandatory that dermatophytes be present and proliferating in the screening culture before a participant could be included in the study, especially for those who were on antifungal therapies

Exclusion criteria: Pregnant or lactating women; those with pre-existing renal, hepatic or gastrointestinal disease, bacterial or yeast infections of the nails or the periungual area, psoriasis and psoriatic changes of the toenail were excluded from the study

Disease duration: Unknown

Comparable at baseline: The distribution of age, sex, height and weight were identical in both treatment groups

Causative species: Nailcare: T. rubrum 13/46 (28.26\%), T. mentagrophytes 8/46 (17.39\%). Placebo: T. rubrum 15/46 (32.60\%), T. mentagrophytes 7/46 (15.21\%)

Number of people lost to follow-up: Nailcare $n=5$, placebo $n=5$; all due to non-compliance (not attending appointments)

Trial duration: unknown

Treatment duration; 2 capsules and cream twice daily for 12 weeks

Drug in study arm 1: NailCare (capsule and cream, multiple herbs in medication including Commiphora mukul, Ocimum basilicum and Vetiveria zizanoides), $\mathrm{n}=21$

Drug in study arm 2: Placebo (capsule and cream), $\mathrm{n}=22$

\begin{tabular}{ll}
\hline Outcomes & Mycological cure (KOH and culture) at weeks 2, 4, 6, 8, 10 and 12 \\
& Adverse events: not reported
\end{tabular}

Notes

No explicit statements of conflicts of interest. Affiliation of 1 of 2 authors is industry. Data extracted were a subset of the participants randomised; we used data from participants that were positive for mycology at baseline

\section{Risk of bias}

Bias Authors' judgement Support for judgement

\begin{tabular}{|c|c|c|}
\hline $\begin{array}{l}\text { Random sequence genera- } \\
\text { tion (selection bias) }\end{array}$ & Low risk & $\begin{array}{l}\text { Quote: "randomly assigned to treatment...according to a computer generated } \\
\text { randomisation schedule" } \\
\text { Comment: Specific randomisation method that is suitable was specified }\end{array}$ \\
\hline $\begin{array}{l}\text { Allocation concealment } \\
\text { (selection bias) }\end{array}$ & Unclear risk & Comment: No information was available. \\
\hline $\begin{array}{l}\text { Blinding of participants } \\
\text { and personnel (perfor- } \\
\text { mance bias) } \\
\text { All outcomes }\end{array}$ & Low risk & $\begin{array}{l}\text { Quote: "identical placebo capsule and cream" } \\
\text { Comment: Blinding was appropriate }\end{array}$ \\
\hline
\end{tabular}

\begin{tabular}{lll}
\hline $\begin{array}{l}\text { Blinding of outcome as- } \\
\text { sessment (detection bias) } \\
\text { All outcomes }\end{array}$ & Unclear risk & \\
\hline $\begin{array}{l}\text { Incomplete outcome data } \\
\text { (attrition bias) }\end{array}$ & Low risk & $\begin{array}{l}\text { Comment: Reasons for participants discontinuing the study were provided for } \\
\text { All outcomes }\end{array}$
\end{tabular}


Kumar 2001 (Continued)

Selective reporting (re- Unclear risk Comment: Review authors were unable to find a clinical trial register entry for porting bias) this study

Other bias

Low risk

Comment: Not a multiple intervention trial, all outcomes reported for interventions

Lahfa 2013

\begin{tabular}{|c|c|}
\hline \multirow[t]{9}{*}{ Methods } & RCT (parallel) \\
\hline & Study design: Open label (evaluator blind) \\
\hline & Sample size calculation: Yes \\
\hline & Diagnosis mycology: $\mathrm{KOH}$ and culture \\
\hline & Big toenail specified: Yes \\
\hline & Statistical comparisons for outcome: Yes \\
\hline & Duration of follow-up: At 4 weeks \\
\hline & Time points of measurements: Days 0, 21, 77 and 105 \\
\hline & $\begin{array}{l}\text { Location: France (9 sites), Belgium ( } 4 \text { sites), Italy ( } 3 \text { sites), and Poland (10 sites) (cities/institutions not } \\
\text { stated) }\end{array}$ \\
\hline \multirow[t]{10}{*}{ Participants } & Total n: 105 \\
\hline & Age: Mean \pm SD (range): $40 \%$ urea ointment $54.3 \pm 14.9$ years, bifonazole-urea $52.4 \pm 14.8$ years, $(18<)$ \\
\hline & $\begin{array}{l}\text { Sex: Urea ointment: men 33/53 (62.3\%), women 20/53 (37.7\%). Bifonazole-Urea: men 33/52 (63.5\%), } \\
\text { women } 19 / 52(36.5 \%)\end{array}$ \\
\hline & $\begin{array}{l}\text { Inclusion criteria: The study included hospital and private outpatients aged } 18 \text { years or older with a } \\
\text { diagnosis of onychomycosis of the great toenail. Clinical diagnosis of distal-lateral or lateral subun- } \\
\text { gual onychomycosis of } 1 \text { great toenail (the target nail) was required. The target nail plate had to show } \geq \\
12.5 \% \text { of the clinically-infected area and have at least } 2 \mathrm{~mm} \text { of the proximal target nail free of infection. } \\
\text { The target nail infection was exclusively from a dermatophyte, as demonstrated both by positive direct } \\
\text { microscopy and by positive fungal culture as reported by the central mycological laboratory }\end{array}$ \\
\hline & $\begin{array}{l}\text { Exclusion criteria: People with more than } 2 \text { infected toenails, people with moccasin-type tinea pedis, } \\
\text { and those suffering from psoriasis, lichen planus, or other abnormalities that could result in clinical- } \\
\text { ly abnormal toenail(s). Peopls who had received systemic antifungal therapy or topical antifungal nail } \\
\text { lacquer within } 3 \text { months or any other topical antifungal therapy applied to the feet or toenails within } 2 \\
\text { months prior to the screening visit were excluded. Systemic corticosteroids, systemic antifungal treat- } \\
\text { ment, and any topical treatment applied directly to the feet or the treated nail other than the investiga- } \\
\text { tional products provided were prohibited throughout the study }\end{array}$ \\
\hline & $\begin{array}{l}\text { Disease duration: Mean } \pm \text { SD: urea ointment with plastic dressing } 4.1 \pm 4.3 \text { years. Bifonazole urea } 6.3 \pm \\
7.4 \text { years }\end{array}$ \\
\hline & $\begin{array}{l}\text { Comparable at baseline: There were no relevant differences between treatment groups in baseline } \\
\text { characteristics }\end{array}$ \\
\hline & Causative species: dermatophyte \\
\hline & Number of people lost to follow-up: $n=5$ in treatment, $n=4$ in bifonazole urea \\
\hline & Trial duration: unknown \\
\hline
\end{tabular}


Lahfa 2013 (Continued)

Interventions

Treatment duration: daily for 3 weeks, followed by 8 weeks of bifonazole cream (both arms)

Drug in study arm 1: 40\% Urea ointment with plastic dressing, $n=53$

Drug in study arm 2: $40 \%$ urea and $1 \%$ bifonazole, $n=52$

\begin{tabular}{ll}
\hline Outcomes & Mycological cure ( $\mathrm{KOH}$ and culture) at days $0,21,77$ and 105 \\
& Complete cure (mycological cure and clinical cure) at days $0,21,77$ and 105 \\
& Clinical cure (no residual clinical signs of onychomycosis) at days $0,21,77$ and 105 \\
& Adverse events: reported \\
\hline Notes & $\begin{array}{l}\text { 3 of } 12 \text { authors are employees of industry, with an additional } 3 \text { authors having ties to industry (e.g. con- } \\
\text { sultant, investigator). The study was sponsored by industry }\end{array}$
\end{tabular}

\section{Risk of bias}

\begin{tabular}{lll}
\hline Bias & Authors' judgement & Support for judgement \\
\hline $\begin{array}{ll}\text { Random sequence genera- } \\
\text { tion (selection bias) }\end{array}$ & Low risk & $\begin{array}{l}\text { Quote: "randomly assigned [to treatment] according to a centralized randomi- } \\
\text { sation table" }\end{array}$ \\
& & Comment: Specific randomisation method that is suitable was specified \\
\hline
\end{tabular}

\begin{tabular}{ll}
\hline $\begin{array}{l}\text { Allocation concealment } \\
\text { (selection bias) }\end{array}$ & Unclear risk \\
\hline
\end{tabular}

Blinding of participants $\quad$ High risk Comment: This was an open-label study
and personnel (perfor-
mance bias)
All outcomes

\begin{tabular}{|c|c|c|}
\hline \multirow[t]{2}{*}{$\begin{array}{l}\text { Blinding of outcome as- } \\
\text { sessment (detection bias) } \\
\text { All outcomes }\end{array}$} & Low risk & $\begin{array}{l}\text { Quote: "centrally assessed by a blinded dermatologist expert panel...Derma- } \\
\text { tologists from the panel were not investigators in the study and were totally } \\
\text { blinded regarding treatment allocation" }\end{array}$ \\
\hline & & Comment: Blinding was appropriate \\
\hline
\end{tabular}

\begin{tabular}{lll}
\hline $\begin{array}{l}\text { Incomplete outcome data } \\
\text { (attrition bias) } \\
\text { All outcomes }\end{array}$ & High risk & Comment: Reasons for attrition were not provided \\
\hline $\begin{array}{l}\text { Selective reporting (re- } \\
\text { porting bias) }\end{array}$ & Low risk & $\begin{array}{l}\text { Comment: Efficacy outcomes stated in trial protocol reported in publication. } \\
\text { NCT00808366 }\end{array}$ \\
\hline Other bias & Low risk & $\begin{array}{l}\text { Comment: Not a multiple intervention trial, all outcomes reported for inter- } \\
\text { ventions }\end{array}$ \\
\hline
\end{tabular}

Lu 2005

$\begin{array}{ll}\text { Methods } & \text { RCT (parallel) } \\ \text { Study design: Double blind } \\ \text { Sample size calculation: Unknown }\end{array}$


Lu 2005 (Continued)

Diagnosis mycology: $\mathrm{KOH}$ and culture

Big toenail specified: Yes

Statistical comparisons for outcome: Yes

Duration of follow-up: At 12 weeks

Time points of measurements: 12 weeks

Location: China (city/institution not stated)

\begin{tabular}{l} 
Total $\mathrm{n}: 120$ \\
Age: 18 - 65 years of age \\
Sex: Unknown \\
Inclusion criteria: 18 - 65 years of age, with mycologically ( $\mathrm{KOH}$ and culture) confirmed dermatophyte \\
onychomycosis involving $>25 \%$ of at least 1 great toenail \\
Exclusion criteria: Unknown \\
Disease duration: Unknown \\
Comparable at baseline: Unknown \\
Causative species: dermatophyte \\
Number of people lost to follow-up: $\mathrm{n}=3$, treatment unknown \\
Trial duration: unknown \\
\hline
\end{tabular}

Interventions

Drug in study arm 1: $0 \%$ terbinafine $\mathrm{HCl}$ lacquer once daily for 12 weeks, $\mathrm{n}=$ unknown

Drug in study arm 2: $1 \%$ terbinafine $\mathrm{HCl}$ lacquer once daily for 6 weeks, $\mathrm{n}=$ unknown

Drug in study arm 3: 1\% terbinafine $\mathrm{HCl}$ lacquer once daily for 12 weeks, $\mathrm{n}=$ unknown

Drug in study arm 4: 5\% terbinafine $\mathrm{HCl}$ lacquer once daily for 6 weeks, $n=$ unknown

Drug in study arm 5: 5\% terbinafine $\mathrm{HCl}$ lacquer once daily for 12 weeks, $\mathrm{n}=$ unknown

Drug in study arm 6: 10\% terbinafine $\mathrm{HCl}$ lacquer once daily for 6 weeks, $\mathrm{n}=$ unknown

Drug in study arm 7: 10\% terbinafine $\mathrm{HCl}$ lacquer once daily for 12 weeks, $\mathrm{n}=$ unknown

Drug in study arm 8: ciclopirox $8 \%$ lacquer once daily for 12 weeks, $n=$ unknown

Mycological outcomes: negative $\mathrm{KOH}$ and negative culture at 12 weeks
Clinical success (negative $\mathrm{KOH}$, negative culture, new unaffected nail length $\geq 3 \mathrm{~mm}$ ) at 12 weeks
Adverse events: reported

\section{Risk of bias}

\section{Bias}

Random sequence generation (selection bias) 
Lu 2005 (Continued)

Allocation concealment $\quad$ Unclear risk Comment: No information was available
(selection bias)

Blinding of participants $\quad$ Unclear risk
and personnel (perfor-
mance bias)

\begin{tabular}{|c|c|c|}
\hline $\begin{array}{l}\text { Blinding of outcome as- } \\
\text { sessment (detection bias) } \\
\text { All outcomes }\end{array}$ & Unclear risk & Comment: No information was available \\
\hline $\begin{array}{l}\text { Incomplete outcome data } \\
\text { (attrition bias) } \\
\text { All outcomes }\end{array}$ & High risk & $\begin{array}{l}\text { Comment: Three patients were not included in the efficacy analysis, no rea- } \\
\text { sons or treatment groups provided }\end{array}$ \\
\hline $\begin{array}{l}\text { Selective reporting (re- } \\
\text { porting bias) }\end{array}$ & Unclear risk & $\begin{array}{l}\text { Comment: Review authors were unable to find a clinical trial register entry for } \\
\text { this study }\end{array}$ \\
\hline Other bias & Low risk & $\begin{array}{l}\text { Comment: Multiple intervention trial however, outcomes for all interventions } \\
\text { reported separately }\end{array}$ \\
\hline
\end{tabular}

Montana 1994

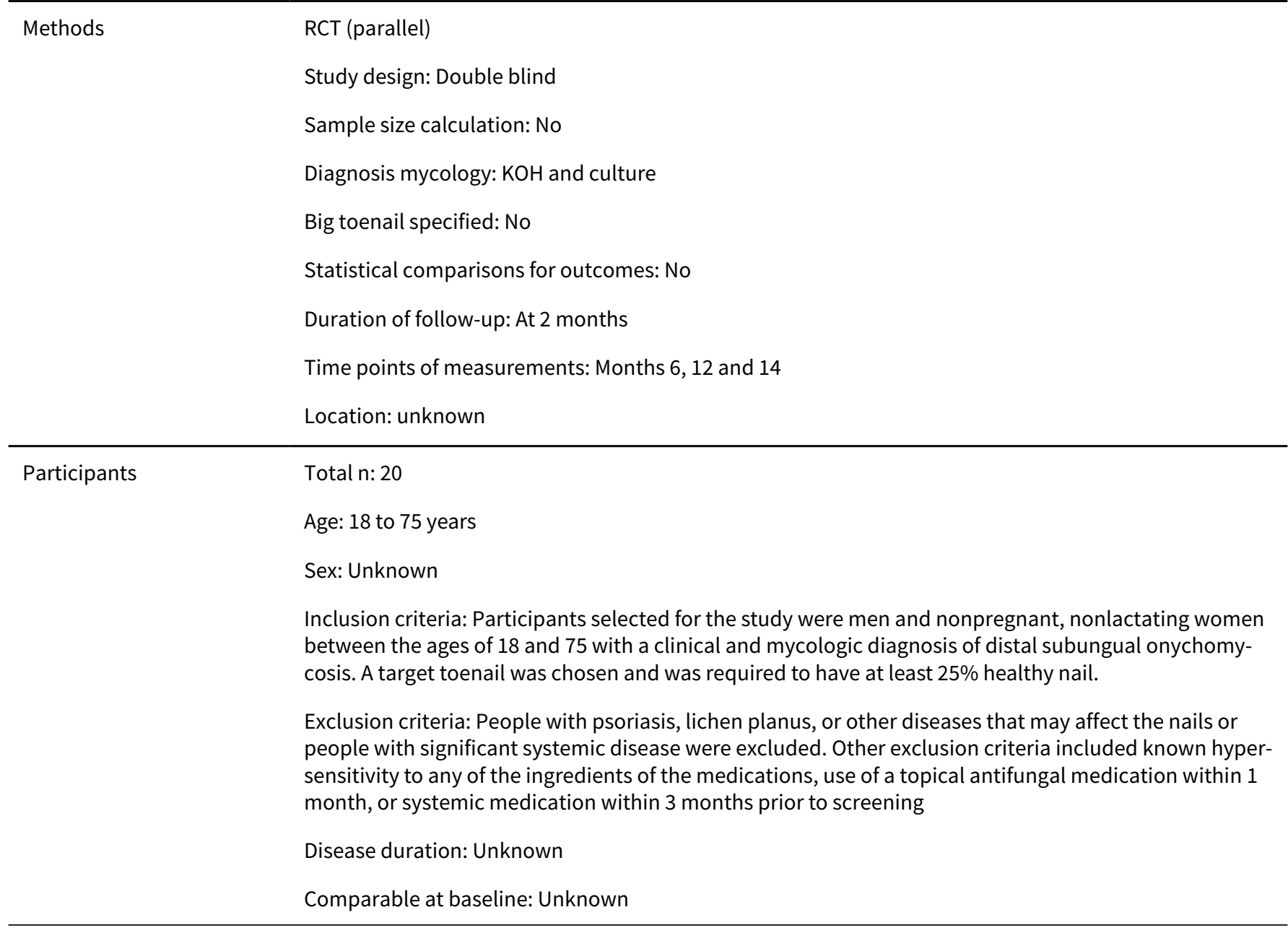


Number of participants lost to follow-up: lack of efficacy of drug: $n=1$, nondermatophyte infection (treatment failed): $\mathrm{n}=1$; study arm unknown

Trial duration: unknown

\begin{tabular}{ll}
\hline Interventions & Treatment duration: twice daily for 12 months \\
& $\begin{array}{l}\text { Drug in study arm 1: Active Group fungoid tincture (triacetin, cetylpyridinium chloride and chlorox- } \\
\text { ylenol), } \mathrm{n}=10 .\end{array}$ \\
& Drug in study arm 2: Vehicle, $\mathrm{n}=10$ \\
\hline Outcomes & Mycological cure (KOH and culture) at months 6,12 and 14 \\
& Adverse events: reported \\
\hline Notes & Quote: "This study was supported by a research grant from [industry], which supplied all test materi- \\
& als."
\end{tabular}

\section{Risk of bias}

\begin{tabular}{lll}
\hline Bias & Authors' judgement & Support for judgement \\
\hline $\begin{array}{l}\text { Random sequence genera- } \\
\text { tion (selection bias) }\end{array}$ & Unclear risk & $\begin{array}{l}\text { Quote: "Patients were randomly assigned" } \\
\text { Comment: Method of randomisation not specified }\end{array}$ \\
\hline $\begin{array}{l}\text { Allocation concealment } \\
\text { (selection bias) }\end{array}$ & Unclear risk & Comment: No information was available \\
\hline $\begin{array}{l}\text { Blinding of participants } \\
\text { and personnel (perfor- } \\
\text { mance bias) }\end{array}$ & Unclear risk & $\begin{array}{l}\text { Quote: "Neither the investigator nor the patients knew the type of medication } \\
\text { that each patient received" }\end{array}$ \\
All outcomes & $\begin{array}{l}\text { Comment: Appears to be blinded, but no indication if treatments looked iden- } \\
\text { tical }\end{array}$
\end{tabular}

\begin{tabular}{lll}
\hline $\begin{array}{l}\text { Blinding of outcome as- } \\
\text { sessment (detection bias) }\end{array}$ & Unclear risk & $\begin{array}{l}\text { Comment: Blinded, but no specific mention of outcome assessor blinding } \\
\text { method or who performed assessments }\end{array}$
\end{tabular}

All outcomes

$\begin{array}{ll}\begin{array}{l}\text { Incomplete outcome data } \\ \text { (attrition bias) }\end{array} \text { High risk } & \begin{array}{l}\text { Quote: "Two patients did not complete the study for reasons unrelated to } \\ \text { the drug trial. They were replaced by two additional patients. One patient } \\ \text { dropped out due to lack of efficacy of the drug. One of the patients...had a } \\ \text { nondermatophyte infection" }\end{array} \\ & \begin{array}{l}\text { Comment: Treatment allocation of participants who did not complete the } \\ \text { study not specified }\end{array}\end{array}$

\begin{tabular}{|c|c|c|}
\hline $\begin{array}{l}\text { Selective reporting (re- } \\
\text { porting bias) }\end{array}$ & High risk & $\begin{array}{l}\text { Comment: Clinical outcomes are not defined clearly or reported. Study pre- } \\
\text { dates trial registry in the USA }\end{array}$ \\
\hline
\end{tabular}

\begin{tabular}{ll}
\hline Other bias $\quad$ Low risk & $\begin{array}{l}\text { Comment: Not a multiple intervention trial, all outcomes reported for inter- } \\
\text { ventions }\end{array}$
\end{tabular}


NCT01080079

Rethods (parallel)
Study design: Single blind (participant)
Sample size calculation: Unknown
Diagnosis mycology: KOH and culture
Big toenail specified: Yes
Statistical comparisons for outcomes: Unknown
Duration of follow-up: 11 months
Time points of measurements: Month 11
Location: University of Alabama at Birmingham, Birmingham, Alabama, United States; International
Clinical Research, LLC, Sanford, Florida, United States; Gwinnett Clinical Research Center, Inc., Snel-
Iville, Georgia, United States; Oregon Dermatology and Research Center, Portland, Oregon, United
States; Temple University School of Podiatric Medicine, Philadelphia, Pennsylvania, United States; Ten-
nessee Clinical Research Center, Nashville, Tennessee, United States; Endeavor Clinical Trials, PA, San
Antonio, Texas, United States; Mediprobe Research Inc., London, Ontario, Canada; Lynderm Research
Inc., Markham, Ontario, Canada

Participants Total n: 168

Age: 18 - 69 years of age

Sex: Men and women (ratio unknown)

Inclusion criteria: Clinical diagnosis of distal subungual onychomycosis of at least 1 great toenail with $25 \%-65 \%$ nail involvement inclusive, positive $\mathrm{KOH}$ microscopy and culture for a dermatophyte, nail plate $\leq 3 \mathrm{~mm}$ thick. Willingness to refrain from using any nail polish products, oral and topical antifungals (except as provided by the investigator for active tinea pedis), and avoid pregnancy

Exclusion criteria: Proximal subungual or white superficial onychomycosis, lunula involvement, psoriasis, eczema, tinea pedis, lichen planus, nail abnormalities, dermatophytoma or spikes, uncontrolled diabetes mellitus or diabetes requiring pharmaceutical therapy, peripheral vascular disease, immunosuppression, topical antifungals or corticosteroids in preceding 2 weeks, systemic corticosteroids within 30 days, systemic antifungals within 120 days, pregnant or nursing women, inappropriate birth control, pacemaker or automatic implantable cardioverter/defibrillator, implantable electronic devices

Disease duration: Unknown

Comparable at baseline: Unknown

Causative species: dermatophytes

Number of people lost to follow-up: Unknown

Trial duration: May 2010 - June 2012

Interventions

Drug in study arm 1: terbinafine hydrochloride gel $4 \%$ with iontophoresis, $n=$ unknown

Drug in study arm 2: placebo with iontophoresis, $\mathrm{n}=$ unknown

Outcomes Clinical and microbiological improvement at 11 months

Adverse events: not reported

Notes

Commercial sponsor. Industry funding. Last update posted on clinicaltrials.gov: October 19, 2012

\section{Risk of bias}


NCT01080079 (Continued)

\begin{tabular}{lll} 
Bias & Authors' judgement & Support for judgement \\
\hline $\begin{array}{l}\text { Random sequence genera- } \\
\text { tion (selection bias) }\end{array}$ & Unclear risk & $\begin{array}{l}\text { Comment: "randomised" } \\
\text { Comment: Method of randomisation not sped }\end{array}$ \\
\hline $\begin{array}{l}\text { Allocation concealment } \\
\text { (selection bias) }\end{array}$ & Unclear risk & Comment: No information in trial registry \\
\hline $\begin{array}{l}\text { Blinding of participants } \\
\text { and personnel (perfor- } \\
\text { mance bias) } \\
\text { All outcomes }\end{array}$ & Unclear risk & Comment: No information in trial registry \\
\hline
\end{tabular}

\begin{tabular}{lll}
\hline $\begin{array}{l}\text { Blinding of outcome as- } \\
\text { sessment (detection bias) } \\
\text { All outcomes }\end{array}$ & Unclear risk & Comment: No information in trial registry \\
\hline $\begin{array}{l}\text { Incomplete outcome data } \\
\text { (attrition bias) } \\
\text { All outcomes }\end{array}$ & Unclear risk & Comment: No information in trial registry \\
\hline $\begin{array}{l}\text { Selective reporting (re- } \\
\text { porting bias) }\end{array}$ & High risk & Quote: "Clinical and microbiological improvement in nails" \\
& $\begin{array}{l}\text { Comment: Outcomes provided in clinical trial register (e.g. clinical improve- } \\
\text { ment in nails) not defined }\end{array}$ \\
\hline Other bias & Unclear risk & Comment: There are no results to assess \\
\hline
\end{tabular}

NCT01145807

Rethods (parallel)
Study design: Double blind
Sample size calculation: Unknown
Diagnosis mycology: $\mathrm{KOH}$ and culture
Big toenail specified: Yes
Statistical comparisons for outcomes: Unknown
Duration of follow-up: 12 weeks
Time points of measurements: Week 48, 52, and 60
Location: Academic Dermatology Associates, Alberquerque, New Mexico, United States
Total n: 738
Age: 18 - 75 years of age
Sex: Men and women (ratio unknown)
Inclusion criteria: Clinical diagnosis of distal subungual onychomycosis of at least 1 great toenail with
$25 \%$ - $65 \%$ nail involvement inclusive, positive KOH microscopy and culture for a dermatophyte, cul-
ture for a dermatophyte, or culture of dermatophyte/nondermatophyte. Target nail must have capaci-


NCT01145807 (Continued)

ty for growth as determined by a history of nail cutting. Willingness to refrain from using any nail polish products and willingness to refrain from pedicures for duration of study

Exclusion criteria: Use of an investigational drug within 1 month of study, pregnancy or planning to become pregnant, lactation, hypersensitivity to terbinafine, inability to self-apply study medication, symptomatic tinea pedis requiring treatment, oral terbinafine within 6 months of study start, oral antifungals within 3 months, topical antifungals within 1 month of study start for toes or feet, nail dystrophy interfering with the assessment of clear nail, superficial white or proximal subungual onychomycosis, non-dermatophyte-only infections, matrix involvement, nail plate thickness $>2 \mathrm{~mm}$, yellow streaks or dermatophytoma, history of peripheral arterial disease or diabetes mellitus, ALT or AST levels $>2$ times the upper limit of normal without clinical reason

Disease duration: Unknown

Comparable at baseline: Unknown

Causative species: dermatophytes or mixed dermatophytes-nondermatophyte

Number of people lost to follow-up: Unknown

Trial duration: April 2010 - August 2012
Treatment duration: Twice daily to all nails for 48 weeks

Drug in study arm 1: TDT 067 terbinafine in transfersome spray, $n=$ unknown

Drug in study arm 2: Sham comparator, transfersome spray, $\mathrm{n}=$ unknown

Drug in study arm 3: Placebo comparator, non-transfersome spray, $\mathrm{n}=$ unknown

Complete cure at weeks 48, 52, 60
Mycologic cure at weeks 48, 52, 60
Effective treatment at weeks 48, 52, 60
Adverse events: not reported

Notes

Commercial sponsor. Industry funding. Last update posted on clinicaltrials.gov: August 1, 2012

\section{Risk of bias}

\begin{tabular}{lll}
\hline Bias & Authors' judgement & Support for judgement \\
\hline $\begin{array}{l}\text { Random sequence genera- } \\
\text { tion (selection bias) }\end{array}$ & Unclear risk & $\begin{array}{l}\text { Comment: "randomised" } \\
\text { Comment: Method of randomisation not specified }\end{array}$ \\
\hline $\begin{array}{l}\text { Allocation concealment } \\
\text { (selection bias) }\end{array}$ & Unclear risk & Comment: No information in trial registry \\
\hline $\begin{array}{l}\text { Blinding of participants } \\
\text { and personnel (perfor- } \\
\text { mance bias) } \\
\text { All outcomes }\end{array}$ & Unclear risk & Comment: No information in trial registry \\
\hline
\end{tabular}

\begin{tabular}{lll}
\hline Blinding of outcome as- & Unclear risk & Comment: No information in trial registry \\
sessment (detection bias) &
\end{tabular}

All outcomes

Incomplete outcome data Unclear risk Comment: No information in trial registry
(attrition bias)


NCT01145807 (Continued)

All outcomes

\begin{tabular}{|c|c|c|}
\hline $\begin{array}{l}\text { Selective reporting (re- } \\
\text { porting bias) }\end{array}$ & High risk & $\begin{array}{l}\text { Comment: Outcomes provided in clinical trial register (e.g. complete cure, ef- } \\
\text { fective treatment rate) not defined }\end{array}$ \\
\hline
\end{tabular}

Other bias Unclear risk Comment: Multiple treatment arm study, but there are no results to assess

\section{NCT01246518}

$\begin{array}{ll}\text { Methods } & \text { RCT (parallel) } \\ \text { Study design: Open } \\ \text { Sample size calculation: Unknown } \\ \text { Diagnosis mycology: Culture } \\ \text { Big toenail specified: Yes } \\ \text { Statistical comparisons for outcomes: Unknown } \\ \text { Duration of follow-up: } 9 \text { and } 3 \text { months } \\ \text { Time points of measurements: } 12 \text { months } \\ \text { Location: } 15 \text { sites, Sweden (cities/institutions unknown) }\end{array}$

Participants

Total n: 250

Age: 18 - 70 years of age

Sex: Men and women (ratio unknown)

Inclusion criteria: Clinical diagnosis of distal subungual onychomycosis (DSO) of at least 1 great toenail affecting $25 \%-75 \%$ nail, positive culture for a dermatophyte

Exclusion criteria: Proximal subungual onychomycosis, DSO of both great toenails where unaffected proximal nail is less than $2 \mathrm{~mm}$, spikes, dermatophytoma, oral and topical antifungal use within 3 and 1 month of screening, respectively, signs of severe peripheral circulatory insufficiency, immunosuppression, participation in a clinical trial during the previous 4 weeks, known allergies to treatment products, pre-menopausal women who are pregnant or nursing, not surgically sterile, or not practicing acceptable birth control

Disease duration: Unknown

Comparable at baseline: Unknown

Causative species: dermatophytes

Number of people lost to follow-up: Unknown

Trial duration: December 2010 - October 2012

Interventions

Drug in study arm 1: MOB015 once a day for 3 months, $\mathrm{n}=$ unknown

Drug in study arm 2: MOB015 once a day for 9 months, $\mathrm{n}=$ unknown

Outcomes Mycologic cure (negative microscopy and culture) at 12 months

Proportion of participants with negative culture, negative microscopy, and complete cure at 1, 3, 6, 9, and 12 months 
NCT01246518 (Continued)

Adverse events: not reported

Notes

Industry provided financial support. Last update posted on clinicaltrials.gov: 25 October 2012; Email correspondence: Data are not available. Phase 3 trials with this agent are ongoing, NCT02859519 and NCT02866032

\section{Risk of bias}

Bias Authors' judgement Support for judgement

Random sequence genera- Low risk tion (selection bias)
Email response: "randomisation using the method of randomly permuted blocks."

Comment: Method of randomisation appropriate

\begin{tabular}{lll}
\hline $\begin{array}{l}\text { Allocation concealment } \\
\text { (selection bias) }\end{array}$ & Low risk & $\begin{array}{l}\text { Email response: "randomisation lists will be prepared by [a third party]...group } \\
\text { will be allocated in the web-based e-CRF provided by [the third party]" } \\
\text { Comment: Allocation concealment appropriate }\end{array}$ \\
\hline $\begin{array}{l}\text { Blinding of participants } \\
\begin{array}{l}\text { and personnel (perfor- } \\
\text { mance bias) }\end{array}\end{array}$ & High risk & Comment: Open-label study \\
All outcomes & \\
\hline
\end{tabular}

\begin{tabular}{lll}
\hline $\begin{array}{l}\text { Blinding of outcome as- } \\
\text { sessment (detection bias) } \\
\text { All outcomes }\end{array}$ & High risk & Comment: Open-label study \\
\hline $\begin{array}{l}\text { Incomplete outcome data } \\
\text { (attrition bias) } \\
\text { All outcomes }\end{array}$ & Unclear risk & Comment: No information in trial registry \\
\hline $\begin{array}{l}\text { Selective reporting (re- } \\
\text { porting bias) }\end{array}$ & High risk & $\begin{array}{l}\text { Comment: Outcome (complete cure) provided in clinical trial register not de- } \\
\text { fined }\end{array}$ \\
\hline Other bias & Unclear risk & $\begin{array}{l}\text { Comment: Not a multiple treatment arm study, but there are no results to as- } \\
\text { sess }\end{array}$ \\
\hline
\end{tabular}

\section{NCT01400594}

\begin{tabular}{|c|c|}
\hline \multirow[t]{9}{*}{ Methods } & $\mathrm{RCT}$ (parallel) \\
\hline & Study design: Double blind \\
\hline & Sample size calculation: Unknown \\
\hline & Diagnosis mycology: $\mathrm{KOH}$ and culture \\
\hline & Big toenail specified: Yes \\
\hline & Statistical comparisons for outcomes: Unknown \\
\hline & Duration of follow-up: Unknown \\
\hline & Time points of measurements: Week 48 \\
\hline & Locations: 37 sites in the United States \\
\hline
\end{tabular}


(Birmingham, Alabama, 3 sites: Total Skin and Beauty Dermatology Center; Radiant Research, Inc; UAB Department of Dermatology, University of Alabama; Clinical Research Advantage, Inc., Glendale, Arizona; Radiant Research, Inc., Tucson, Arizona; Diagnamics, Inc. Encinatas, California; Radiant Research, Inc., Denver, Colorado; Center for Clinical and Cosmetic Research, Aventura, Florida; Marta Rendon, MD, Skin Care Research, Inc., Boca Raton, Florida; Pab Clinical Research, Brandon, Florida; Dermatology Research Institute, Coral Gables, Florida; Ameriderm Research, Jacksonville, Florida; Lake Washington Foot \& Ankle Center, Melbourne, Florida; Florida Academic Dermatology Center; Miami, Florida; Ameriderm Research, Ormond Beach, Florida; Leavitt Medical Associates of FL, Ormond Beach, Florida; Radiant Research, Inc., Pinellas Park, Florida; Radiant Research, Inc., Atlanta, Georgia; Radiant Research, Inc., Chicago, Illinois; Dawes Fretzin Clinical Research Group, LLC, Indianapolis, Indiana; Derm Research, LLC, Louisville, Kentucky; William P Coleman III, MD, Metairie, Louisiana; Callender center for Clinical Research, Glenn Dale, Maryland; Assoc Foot Clinic \& Surgery Specialists, Flint, Michigan; Silverton Skin Institute, Grand Blanc, Michigan; University of Minnesota, Minnesota Clinical Study Center, Fridley, Minnesota; Radiant Research, St. Louis, Missouri; Radiant Research, Inc., Akron, Ohio; Group Health Associates, Tri-Health, Cincinnati, Ohio; Central Sooner Research, Norman, Oklahoma; Society Hill Dermatology, Philadelphia, Pennsylvania; University of Pittsburg Medical Center, Department of Dermatology, Pittsburgh, Pennsylvania; Radiant Research, Inc., Anderson, South Carolina; Radiant Research, Inc., Dallas, Texas; Radiant Research, Inc., San Antonio, Texas; Coastal Podiatry Group, Virginia Beach, Virginia)

\section{Participants} Total n: 182

Age: 18 - 70 years of age

Sex: Men and women (ratio unknown)

Inclusion criteria: Clinical diagnosis of distal subungual onychomycosis of at least 1 great toenail, positive $\mathrm{KOH}$ microscopy and culture for a dermatophyte, good general health, willingness to refrain from using lotion, cream, liquid, or polish on treated nails, willingness to refrain from pedicures, acceptable birth control

Exclusion criteria: Inability to self-apply test product onto toenails, use of topical antifungals within last month, uncontrolled diabetes, fingernail onychomycosis, confirmed non-dermatophyte infection of target toenail, structural deformities of target toenail, history of severe or chronic immunosuppression, any systemic or dermatologic disorder such as severe eczema or atopic dermatitis, psoriasis of the toenails, hypersensitivity or allergy to topical preparations or adhesive dressings

Disease duration: Unknown

Comparable at baseline: Unknown

Causative species: dermatophytes

Number of people lost to follow-up: Unknown

Trial duration: July 2011 - January 2013

Drug in study arm 1: HTU-520 patch (terbinafine hydrochloride patch), $\mathrm{n}=$ unknown

Drug in study arm 2: Placebo patch, $\mathrm{n}=$ unknown

Adverse events: not reported

\section{Risk of bias}

\section{Bias}

\section{Authors' judgement Support for judgement}




\section{NCT01400594 (Continued)}

Random sequence genera- Unclear risk Quote: "randomised" tion (selection bias)

Comment: Method of randomisation not specified

\begin{tabular}{lll}
\hline $\begin{array}{l}\text { Allocation concealment } \\
\text { (selection bias) }\end{array}$ & Unclear risk & Comment: No information in trial registry \\
\hline $\begin{array}{l}\text { Blinding of participants } \\
\text { and personnel (perfor- } \\
\text { mance bias) }\end{array}$ & Unclear risk & Comment: No information in trial registry \\
All outcomes & & \\
\end{tabular}

Blinding of outcome as- Unclear risk Comment: No information in trial registry sessment (detection bias)

All outcomes

Incomplete outcome data Unclear risk Comment: No information in trial registry
(attrition bias)

(attrition bias)

All outcomes

$\begin{array}{lll}\begin{array}{l}\text { Selective reporting (re- } \\ \text { porting bias) }\end{array} & \text { High risk } & \begin{array}{l}\text { Comment: Outcome provided in clinical trial register not defined (e.g. clinical } \\ \text { cure, mycological cure) }\end{array}\end{array}$

Other bias Unclear risk Comment: Not a multiple treatment study, but there are no results to assess

\section{NCT02022215}

Rethods (parallel)
Study design: Double blind
Sample size calculation: Unknown
Diagnosis mycology: KOH and culture
Big toenail specified: Yes
Statistical comparisons for outcomes: Unknown
Duration of follow-up: 4 weeks
Time points of measurements: Weeks 48, 52
Location: 31 cities in the United States (Birmingham, Alabama; Encinitas, California; San Diego, Cali-
fornia; San Francisco, California; Santa Rosa, California; Denver, Colorado; Miami, Florida; Miramar,
Florida; Newnan, Georgia; Boise, Idaho; Evansville, Indiana; Baltimore, Maryland; Ann Arbor, Michigan;
Clinton Twp, Michigan; Fridley, Minnesota; Albuquerque, New Mexico; New York, New York; Rochester,
New York; Cincinnati, Ohio; Portland, Oregon; Mount Pleasant, South Carolina; Knoxville, Tennessee;
Nashville, Tennessee; Austin, Texas; College Station, Texas; Dallas, Texas; San Antonio, Texas; Salt Lake
City, Utah; Lynchburg, Virginia; Norfolk, Virginia; Spokane, Washington) (institutions unknown)

\footnotetext{
Participants
}

\section{Total n: 304}

Age: 18 - 70 years of age

Sex: Men and women (ratio unknown) 
Inclusion criteria: Men and women aged 18 - 70 years with clinically-diagnosed mild-to-moderate distal subungual onychomycosis of the target nail, with positive $\mathrm{KOH}$ and positive culture for a dermatophyte. Appropriate birth control required and good general health as determined by the investigator

Exclusion criteria: Uncontrolled diabetes, fingernail onychomycosis, prior antifungal drug use with failure to complete washout, history of HIV, hepatitis B or C, diagnosis of psoriasis or history of psoriasis, participation in a trial within 30 days or concurrently, pregnancy/lactation

Disease duration: Unknown

Comparable at baseline: Unknown

Causative species: dermatophytes

Number of people lost to follow-up: Unknown

Trial duration: December 19, 2013 - March 1, 2016

Treatment duration: applied once daily for 48 weeks
Drug in study arm 1: ME1111 solution, low strength, $\mathrm{n}=$ unknown
Drug in study $\operatorname{arm} 2$ : ME1111 solution, high strength, $\mathrm{n}=$ unknown
Drug in study arm 3: Vehicle solution, $\mathrm{n}=$ unknown

Outcomes

Complete cure at week 52

Complete or almost complete cure rate $5 \%$ or less clinical involvement of target great toenail and mycologic cure) at week 52

Number of adverse events up to week 48 , week 52

Local tolerability assessments up to week 48 , week 52

Adverse events: not reported

Notes

Commercial sponsor. Industry funding. Last update posted on clinicaltrials.gov: 23 March 2017

\section{Risk of bias}

\begin{tabular}{|c|c|c|}
\hline Bias & Authors' judgement & Support for judgement \\
\hline \multirow{2}{*}{$\begin{array}{l}\text { Random sequence genera- } \\
\text { tion (selection bias) }\end{array}$} & Unclear risk & Quote: "randomised" \\
\hline & & Comment: Method of randomisation not specified \\
\hline $\begin{array}{l}\text { Allocation concealment } \\
\text { (selection bias) }\end{array}$ & Unclear risk & Comment: No information in trial registry \\
\hline $\begin{array}{l}\text { Blinding of participants } \\
\text { and personnel (perfor- } \\
\text { mance bias) } \\
\text { All outcomes }\end{array}$ & Unclear risk & Comment: No information in trial registry \\
\hline $\begin{array}{l}\text { Blinding of outcome as- } \\
\text { sessment (detection bias) } \\
\text { All outcomes }\end{array}$ & Unclear risk & Comment: No information in trial registry \\
\hline $\begin{array}{l}\text { Incomplete outcome data } \\
\text { (attrition bias) } \\
\text { All outcomes }\end{array}$ & Unclear risk & Comment: No information in trial registry \\
\hline
\end{tabular}


NCT02022215 (Continued)

Selective reporting (re- Low risk Comment: Outcomes provided in clinical trial register comprehensive and porting bias)

Other bias

Unclear risk

NCT02343627

Methods (parallel)
Study design: Double blind
Sample size calculation: Unknown
Diagnosis mycology: KOH and culture
Big toenail specified: No
Statistical comparisons for outcomes: Unknown
Duration of follow-up: 300 days
Time points of measurements: Days $28,60,90,180,270$, and 360
Location: United States (city/institution unknown)

Participants

Total n: 47

Age: $18-65$ years of age

Sex: 41 men (NVXT, $n=30)$ and 6 women (NVXT, $n=5)$

Inclusion criteria: Men and women 18 - 65 years of age with clinically-diagnosed mild-to-moderate onychomycosis of at least 1 toenail, $10 \%-50 \%$ nail involvement without involvement of 1 of the lunular proximal regions, with positive $\mathrm{KOH}$ and positive culture for a dermatophyte. No current topical or systemic antifungal therapy

Exclusion criteria: Nail or anatomical abnormalities of the toe that may interfere with evaluations or dosing compliance

Disease duration: Unknown

Comparable at baseline: Unknown

Causative species: Dermatophytes

Number of people lost to follow-up: NVXT solution, $n=12$; vehicle, $n=1$; reasons not provided

Trial duration: December 2014 - January 2016

Interventions

Treatment duration: applied once daily for 60 days

Drug in study arm 1: NVXT solution, $\mathrm{n}=35$

Drug in study arm 2: Placebo solution, $\mathrm{n}=12$

Outcomes

Negative fungal culture at $28,60,90,180,270$, and 360 days

Mycologic cure (negative $\mathrm{KOH}$ and culture) at 90, 180, 270, and 360 days

Complete cure ( $0 \%$ nail involvement and mycologic cure) at $90,180,270$, and 360 days 
NCT02343627 (Continued)

Therapeutic success (mycologic cure and $\leq 5 \%$ nail involvement or $50 \%$ improvement) at $90,180,270$, and 360 days

Clinical cure ( $0 \%$ nail involvement) at 360 days

Nail involvement (\%) at 360 days

Nail condition/appearance using subjective scale at 90, 180, 270, and 360 days

Adverse events and application site reactions: reported

Notes

Commercial sponsor. Industry funding.Last update posted on clinicaltrials.gov: 22 August 2018

\section{Risk of bias}

\begin{tabular}{|c|c|c|}
\hline Bias & Authors' judgement & Support for judgement \\
\hline \multirow{2}{*}{$\begin{array}{l}\text { Random sequence genera- } \\
\text { tion (selection bias) }\end{array}$} & Unclear risk & Quote: "randomised" \\
\hline & & Comment: Method of randomisation not specified \\
\hline $\begin{array}{l}\text { Allocation concealment } \\
\text { (selection bias) }\end{array}$ & Unclear risk & Comment: No information in trial registry \\
\hline $\begin{array}{l}\text { Blinding of participants } \\
\text { and personnel (perfor- } \\
\text { mance bias) } \\
\text { All outcomes }\end{array}$ & Unclear risk & Comment: No information in trial registry \\
\hline $\begin{array}{l}\text { Blinding of outcome as- } \\
\text { sessment (detection bias) } \\
\text { All outcomes }\end{array}$ & Unclear risk & Comment: No information in trial registry \\
\hline $\begin{array}{l}\text { Incomplete outcome data } \\
\text { (attrition bias) } \\
\text { All outcomes }\end{array}$ & Unclear risk & Comment: No information in trial registry \\
\hline $\begin{array}{l}\text { Selective reporting (re- } \\
\text { porting bias) }\end{array}$ & Low risk & $\begin{array}{l}\text { Comment: Outcomes provided in clinical trial register comprehensive and } \\
\text { well-defined }\end{array}$ \\
\hline Other bias & Unclear risk & $\begin{array}{l}\text { Comment: Not a multiple treatment arm study, but there are no results to as- } \\
\text { sess }\end{array}$ \\
\hline
\end{tabular}

$\begin{array}{ll}\text { Methods } & \text { RCT (parallel) } \\ \text { Study design: Double blind } \\ \text { Sample size calculation: Yes } \\ \text { Diagnosis mycology: } \mathrm{KOH} \text { and culture } \\ \text { Big toenail specified: Yes } \\ \text { Statistical comparison for outcomes: Yes } \\ \text { Duration of follow-up: } 365 \text { days }\end{array}$


Location: Novum Pharmaceutical Research Services (Novum), Pittsburgh, Pennsylvania, United States

Total n: 184
Age: Mean $51.8 \pm 14.2$ years
Sex: Study arm 1: 43 men, 21 women; Study arm 2: 45 men, 17 women; Study arm 3: 37 men, 21 women
Inclusion criteria: Men and women 18 years and older with a clinical diagnosis of mild-to-moderate
onychomycosis of the target toenail (defined as 1 of the infected great toenails with $10 \%-35 \%$ nail in-
volvement). Onset of clinical signs < 2 years before screening, positive KOH microscopy and positive
culture for a dermatophyte, at least 5 mm clear nail between proximal nail fold and deepest extent of
onychomycosis, no lunula involvement.

Exclusion criteria: Women who are pregnant, lactating or likely to become pregnant during the study, people with proximal onychomycosis, dermatophytomas, fungal spikes, limited lateral onychomycosis, white superficial onychomycosis, significant nail dystrophy, total dystrophy, history of current diabetes or peripheral vascular disease or both, distal nail plate thickness $>3 \mathrm{~mm}$, no new nail growth requiring trimming in the last 2 months for target nail, previous treatment for onychomycosis in the last 12 months that was unresponsive to treatment, non-dermatophyte infections, history of nail trauma, current or history of psoriasis or lichen planus within the previous 12 months, immunocompromised, use of topical antifungals, immunomodulators, or systemic corticosteroids on the feet within 4 weeks of baseline and use of topical corticosteroids on the feet within 2 weeks of baseline, use of systemic antifungals for onychomycosis or any antifungals with known activity against dermatophytes within the previous 24 weeks

Disease duration: Unknown

Comparable at baseline: Unknown

Causative species: Dermatophytes

Number of people lost to follow-up: Study arm 1, $n=16$; Study arm 2, $n=14$; Study arm 3, $n=17$

Trial duration: March 21, 2016 - June 21, 2018

Drug in study arm 1: NVXT topical solution daily for 8 weeks, $n=64$

Drug in study arm 2: NVXT topical solution daily for $2 \times 8$-week periods, separated by 32 weeks, $n=62$.

Drug in study arm 3: Placebo topical daily for $2 \times 8$-week periods, separated by 32 weeks, $n=58$

\begin{tabular}{|c|c|}
\hline \multirow[t]{6}{*}{ Outcomes } & Mycological cure (negative $\mathrm{KOH}$ and negative culture) at days 141 and 365 \\
\hline & Clinical cure ( $0 \%$ nail involvement) at days 141,281 , and 365 \\
\hline & Complete cure (clinical and mycological cure) at days 141 and 365 \\
\hline & Almost complete cure ( $\leq 5 \%$ nail involvement and mycological cure) at days 141 and 365 \\
\hline & Almost clinical cure ( $\leq 5 \%$ nail involvement) at days 141,281 , and 365 \\
\hline & Adverse events: reported \\
\hline
\end{tabular}

\section{Risk of bias}




\section{NCT02933879 (Continued)}

Random sequence genera- Low risk tion (selection bias)

Quote: "randomisation will be generated in blocks of 3"

Comment: Specific randomisation method that is suitable was specified

Allocation concealment Low risk
(selection bias)

Quote: "the clinical packaging company will hold the randomisation scheme until database lock."

Comment: Allocation concealment was appropriate

Blinding of participants Low risk

and personnel (perfor-

Low risk

mance bias)

All outcomes
Quote: "The study product will be packaged and blinded by an independent clinical packaging company... The bottles used for all three study products are identical in size, shape and colour. A standard label overlay will be used and there is no difference in odour of the products. Therefore, all three products are indistinguishable."

Comment: Blinding was appropriate
Quote: "The study product will be packaged and blinded by an independent clinical packaging company... The bottles used for all three study products are identical in size, shape and colour. A standard label overlay will be used and there is no difference in odour of the products. Therefore, all three products are indistinguishable."

Comment: Blinding was appropriate

\begin{tabular}{|c|c|c|}
\hline $\begin{array}{l}\text { Incomplete outcome data } \\
\text { (attrition bias) } \\
\text { All outcomes }\end{array}$ & Unclear risk & $\begin{array}{l}\text { Comment: Trial record provides number of people lost to follow-up but does } \\
\text { not provide reasons for discontinuation. Number of people lost is similar } \\
\text { among study arms }\end{array}$ \\
\hline
\end{tabular}

All outcomes

Selective reporting (re- Low risk
porting bias)

Comment: Outcomes are explicit and results are posted in trial registry for outcomes identified in protocol provided as a link in trial registry

\begin{tabular}{ll}
\hline Other bias $\quad$ High risk & $\begin{array}{l}\text { Comment: Multiple intervention trial, but some outcomes are not reported } \\
\text { separately for all interventions }\end{array}$ \\
\hline
\end{tabular}

NCT03141840

RCT (parallel)
Study design: Double blind
Sample size calculation: Unknown
Diagnosis mycology: Unknown
Big toenail specified: Yes
Statistical comparison for outcome: Unknown
Duration of follow-up: 12 months
Time points of measurements: At months 3, 6 and 12 months
Location: Fotcompaniet Stockholm, Stockholm, Sweden

Participants Total n: 69

Age: 18 years and older

Sex: Men and women (ratio unknown) 
Inclusion criteria: Provision of written informed consent, men and women above age 18 weighing over $40 \mathrm{~kg}$, present with distal lateral subungual onychomycosis of any of the big toenails due to dermatophytes, the onychomycosis should involve $<50 \%$ of the nail bed and may not have reached the cuticle

Exclusion criteria: Unable to come for study visits, known allergy to any of the components in ABL01, not willing to participate in the trial or not able to understand the content of the trial, present with proximal subungual onychomycosis or superficial onychomycosis, present with distal lateral subungual onychomycosis of other nails than the big toe, $>50 \%$ of the nailbed affected by onychomycosis or the cuticles infected, known conditions (like psoriasis) that cause abnormal nail appearance, nail damage caused by trauma, pressure or other mechanical reasons, currently on immunosuppressive therapy, showing signs of poor peripheral blood circulation, used another topical antifungal treatment within 1 month of screening, used a systemic antifungal treatment within 3 months of screening, participated in any other clinical onychomycosis trial in the previous 3 months

Disease duration: Unknown

Comparable at baseline: Unknown

Causative species: Dermatophyte

Number of people lost to follow-up: Unknown

Trial duration: Auguts 14, 2017 - February 8, 2019

Interventions Treatment duration: Once weekly for 6 months to 12 months

Intervention in study arm 1: ABL01, $\mathrm{n}=$ unknown

Intervention in study arm 2: Placebo, $\mathrm{n}=$ unknown

Outcomes

Clinical improvement (reduction of $40 \%$ in infected nail area) at 6 months

Clinical improvement (reduction of $80 \%$ in infected nail area) at 12 months

Mycological cure (negative test for dermatophytes) at 12 months

Adverse events: not reported

Participant questionnaire on ease of treatment

\section{Risk of bias}

Bias Authors' judgement Support for judgement

Random sequence genera- Low risk tion (selection bias)

Quote: "Study subjects are randomly assigned to treatment or placebo groups by a predetermined algorithm using sealed envelopes."

Comment: Specific randomisation method that is suitable was specified

\begin{tabular}{ll}
\hline $\begin{array}{l}\text { Allocation concealment } \\
\text { (selection bias) }\end{array}$ & Low risk \\
& $\begin{array}{l}\text { Email response: "Sealed envelopes were supplied by the sponsor which speci- } \\
\text { fied the code indicating the device a patient received...[Investigators] were not } \\
\text { involved in the randomisation procedure, this was handled by the sponsor." }\end{array}$
\end{tabular}

Comment: Allocation concealment was appropriate

\begin{tabular}{|c|c|c|}
\hline $\begin{array}{l}\text { Blinding of participants } \\
\text { and personnel (perfor- } \\
\text { mance bias) }\end{array}$ & Low risk & $\begin{array}{l}\text { Email response: "Placebo and active devices were labelled and packaged iden- } \\
\text { tically...supplied by the sponsor." } \\
\text { Comment: Blinding was adequate }\end{array}$ \\
\hline
\end{tabular}




\section{NCT03141840 (Continued)}

Blinding of outcome assessment (detection bias)

All outcomes

Email response: "The patient opened [and used] the device package at home....no devices present [in the clinic]."

Comment: Blinding was adequate

Comment: No information provided in protocol

Incomplete outcome data Unclear risk

(attrition bias)

All outcomes

\begin{tabular}{lll}
\hline $\begin{array}{l}\text { Selective reporting (re- } \\
\text { porting bias) }\end{array}$ & Low risk & Comment: Outcomes provided in clinical trial register are clearly defined \\
\hline Other bias & Unclear risk & $\begin{array}{l}\text { Comment: Not a multiple treatment arm study, but there are no results to as- } \\
\text { sess }\end{array}$
\end{tabular}

\section{NCT03289871}

Rethods (parallel)
Study design: Open label
Sample size calculation: Unknown
Diagnosis mycology: KOH and culture
Big toenail specified: Yes
Statistical comparisons for outcomes: Unknown
Duration of follow-up: None
Time points of measurements: Days 0, 14, 28, 56, 112, 168
Location: Hospital Habib Thameur, Tunis, Tunisia; Principal Instruction Military Hospital, Tunis, Tunisia

\section{Participants}

\section{Total n: 112}

Age: $18+$ years of age

Sex: Men and women (ratio unknown)

Inclusion criteria: Ability to operate medical device, 18 years of age and older with superficial onychomycosis on at least 1 great toenail or light-to-moderate disto-lateral onychomycosis without matrix involvement, confirmed with $\mathrm{KOH}$ staining, no systemic antifungal within 6 months of study and no topical antifungal within 3 months of study, use of contraception since at least 12 weeks before study until 1 month after the study.

Exclusion criteria: Enrolment in another clinical trial, pregnant, nursing, or women planning to become pregnant, allergy to product constituents, serious or progressive disease such as diabetes, peripheral circulatory disease, HIV, psoriasis, lichen planus, immunosuppression, other cutaneous disease on the nails

Disease duration: Unknown

Comparable at baseline: Unknown

Causative species: Unknown

Number of people lost to follow-up: Unknown 
NCT03289871 (Continued)

Trial duration: January 2015 - November 2015

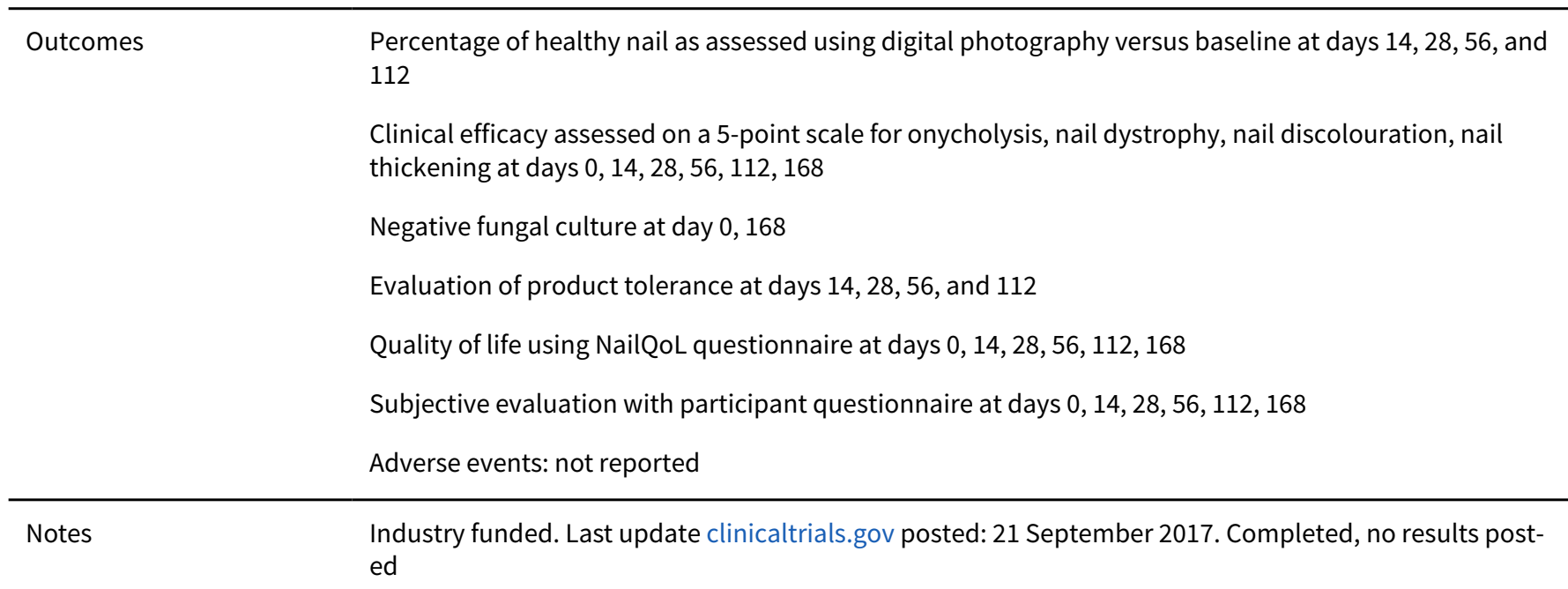

\section{Risk of bias}

\begin{tabular}{|c|c|c|}
\hline Bias & Authors' judgement & Support for judgement \\
\hline $\begin{array}{l}\text { Random sequence genera- } \\
\text { tion (selection bias) }\end{array}$ & Unclear risk & $\begin{array}{l}\text { Quote: "randomised" } \\
\text { Comment: Method of randomisation not specified }\end{array}$ \\
\hline $\begin{array}{l}\text { Allocation concealment } \\
\text { (selection bias) }\end{array}$ & Unclear risk & Comment: No information in trial registry \\
\hline $\begin{array}{l}\text { Blinding of participants } \\
\text { and personnel (perfor- } \\
\text { mance bias) } \\
\text { All outcomes }\end{array}$ & High risk & $\begin{array}{l}\text { Quote: "Masking: None (Open Label)" } \\
\text { Comment: It is likely that participants and personnel were not blinded }\end{array}$ \\
\hline $\begin{array}{l}\text { Blinding of outcome as- } \\
\text { sessment (detection bias) } \\
\text { All outcomes }\end{array}$ & High risk & $\begin{array}{l}\text { Quote: "Masking: None (Open Label)" } \\
\text { Comment: It is likely that outcome assessment was not blinded }\end{array}$ \\
\hline $\begin{array}{l}\text { Incomplete outcome data } \\
\text { (attrition bias) } \\
\text { All outcomes }\end{array}$ & Unclear risk & Comment: No information in trial registry \\
\hline $\begin{array}{l}\text { Selective reporting (re- } \\
\text { porting bias) }\end{array}$ & Low risk & $\begin{array}{l}\text { Comment: Outcomes provided in clinical trial register comprehensive and } \\
\text { well-defined }\end{array}$ \\
\hline Other bias & Unclear risk & $\begin{array}{l}\text { Comment: Not a multiple treatment arm study, but there are no results to as- } \\
\text { sess }\end{array}$ \\
\hline
\end{tabular}

Nijenhuis-Rosien 2019

Methods RCT (parallel)


Nijenhuis-Rosien 2019 (Continued)

\author{
Study design: Quadruple blind \\ Sample size calculation: Yes \\ Diagnosis mycology: Microscopy, culture, and PCR \\ Big toenail specified: Yes \\ Statistical comparisons for outcomes: Yes \\ Duration of follow-up: 1 year
}

Time points of measurement: At weeks 30 and 52

Location: Innofeet, Zwole, The Netherlands

\title{
Participants
}

Total n: 63

Age: Median (IQR) Laser $=70.5(60.7,73.8) ;$ Sham $=66.3(60.9,71.1)$

Sex: Laser, 12 women and 20 men; Sham, 9 women and 22 men

Inclusion criteria: Type 1 or type 2 diabetes, over 18 years old, clinical suspicion with microbiologic confirmation of onychomycosis, participant is at risk for diabetic foot ulcers defines as Simms score 1, 2

Exclusion criteria: People without the microbiological confirmation of fungal nail infection, Sims classification of 0 or 3, people with an active or history of a diabetic foot ulcer, people who used systemic or topical anti fungal agents during the preceding 3 months, people with ischaemic rest pain, people with ankle brachial index $<0.9$, people with a documented toe pressure below $50 \mathrm{mmHg}$, people receiving dialysis, severe renal insufficiency (eGFR below $30 \mathrm{ml} / \mathrm{min}$ ), people with an insufficient knowledge of the Dutch language to understand requirements of the study, people with a dark skin colour (Fitspatrick 4 and 5), people who use immunosuppressive medication, people suffering from nail psoriasis, lichen planus, or other abnormalities that could result in clinically abnormal toenails, and a history of epilepsy

Disease duration: Unknown

Comparable at baseline: Unknown

Causative species: T. rubrum most common, in $78 \%$ of laser participants and $71 \%$ of sham participants. Other dermatophytes and non-dermatophyte moulds present

Number of people lost to follow-up: None

Trial duration: March 2015 - July 2016

Intervention in study arm 2: Sham treatment, $\mathrm{n}=31$

Outcomes

Complete cure (completely normal nail or, negative mycological results where $<5 \%$ target nail affected) at 52 weeks

Microbiologic cure at 52 weeks

Recurrence (same causative species at baseline and week 52, with negative mycology at week 30)

Adverse events: reported

Notes

Not industry funded, foundation grant-funded. One of the employers of the lead author had a stake in a medical device supply company that includes the device in the current study, but the lead author has no stake in this company. All other authors have no conflicts of interest 
Nijenhuis-Rosien 2019 (Continued)

\section{Risk of bias}

\begin{tabular}{|c|c|c|}
\hline Bias & Authors' judgement & Support for judgement \\
\hline \multirow[t]{2}{*}{$\begin{array}{l}\text { Random sequence genera- } \\
\text { tion (selection bias) }\end{array}$} & Low risk & $\begin{array}{l}\text { Quote: "Randomisation was done in blocks ( } 5 \text { blocks of } 10 \text { and } 1 \text { block of 14) } \\
\text { by a third party using sealed, non-transparent envelopes." }\end{array}$ \\
\hline & & Comment: Specific randomisation method that is suitable was specified \\
\hline \multirow[t]{2}{*}{$\begin{array}{l}\text { Allocation concealment } \\
\text { (selection bias) }\end{array}$} & Low risk & $\begin{array}{l}\text { Quote: "The investigators, patients, outcome assessors, and statistician were } \\
\text { blinded for allocation." }\end{array}$ \\
\hline & & $\begin{array}{l}\text { Comment: Randomisation was performed by a third party and not study inves- } \\
\text { tigators. Allocation concealment was appropriate }\end{array}$ \\
\hline \multirow{3}{*}{$\begin{array}{l}\text { Blinding of participants } \\
\text { and personnel (perfor- } \\
\text { mance bias) } \\
\text { All outcomes }\end{array}$} & Low risk & $\begin{array}{l}\text { Quote: "patients were blinded by inserting a hanging barrier (a plain cloth) be- } \\
\text { tween the patients' head and his or her feet. In addition, a blinded laser safe- } \\
\text { ty goggle was worn by the subjects. During the procedure, the laser was ac- }\end{array}$ \\
\hline & & $\begin{array}{l}\text { tually turned on but blasted in a fireproof dish, which was placed next to the } \\
\text { foot. The procedural sounds and lights during the laser application and sham } \\
\text { procedure were identical." "No participant reported knowing about his or her } \\
\text { treatment group." }\end{array}$ \\
\hline & & Comment: Blinding was adequate \\
\hline
\end{tabular}

\begin{tabular}{|c|c|c|}
\hline $\begin{array}{l}\text { Blinding of outcome as- } \\
\text { sessment (detection bias) } \\
\text { All outcomes }\end{array}$ & Low risk & $\begin{array}{l}\text { Comment: Personnel performing treatment were different from study investi- } \\
\text { gators, outcome assessors, and the statistician. Blinding was adequate }\end{array}$ \\
\hline
\end{tabular}

\begin{tabular}{|c|c|c|}
\hline $\begin{array}{l}\text { Incomplete outcome data } \\
\text { (attrition bias) }\end{array}$ & Low risk & $\begin{array}{l}\text { Comment: Reasons for participants discontinuing the study were provided for } \\
\text { each treatment group. The ITT population was clearly specified }\end{array}$ \\
\hline
\end{tabular}

\begin{tabular}{lll}
\hline $\begin{array}{l}\text { Selective reporting (re- } \\
\text { porting bias) }\end{array}$ & Low risk & $\begin{array}{l}\text { Comment: Outcomes reported in published trial protocol are explicit. The } \\
\text { same outcomes are specified and reported verbatim in the publication. } \\
\text { NCT01996995 }\end{array}$ \\
\hline Other bias & Low risk & $\begin{array}{l}\text { Comment: Not a multiple intervention trial, all outcomes reported for inter- } \\
\text { ventions }\end{array}$ \\
\hline
\end{tabular}

Parekh 2017

Rethods (parallel)
Study design: Double blind
Sample size calculation: Yes
Diagnosis mycology: $\mathrm{KOH}$ and culture
Big toenail specified: $\mathrm{No}$
Statistical comparisons for outcomes: Yes
Duration of follow-up: None
Time points of measurements: 6 months


Parekh 2017 (Continued)

Location: Apollo Clinic Bangalore, India

Total $\mathrm{n}: 10$ with onychomycosis
Age: 18 years of age and older
Sex: 20 men and 8 women were initially randomised, sex ratio in final group unknown
Inclusion criteria: Men and women, age 18 and older, diagnosed severe or very severe onychomycosis,
confirmed with positive $\mathrm{KOH}$ smear, positive culture for a dermatophyte, and presence of live spores
Exclusion criteria: Oral or topical tinea treatments 1 week prior to screening, consumption of any drug
1 week prior to, or during, the study that was judged to compromise the study, history of allergy or in-
tolerance to any drug, pregnancy or lactation, participation in a clinical trial within the previous 30
days
Disease duration: Unknown
Comparable at baseline: Yes

Causative species: dermatophytes

Number of people lost to follow-up: None

Trial duration: March 27, 2010 - January 31, 2011

Interventions

Drug in study arm 1: Calmagen ${ }^{\circledR}$ lotion twice daily for 12 weeks, followed by once daily for an additional 12 weeks, $n=5$

Drug in study arm 2: Placebo lotion twice daily for 12 weeks, followed by once daily for an additional 12 weeks, $n=5$

Mycological cure: $\mathrm{KOH}$, culture, and live spore counts at 6 months
Clinical cure: IGA response of cleared or excellent at 6 months
Reduction in severity score of symptoms using SCIO at 6 months
Improvement assessed by photographs at 6 months
Adverse events: reported

Notes

2 of 6 authors own stock in parent company of treatment. No other competing interests listed. The study was sponsored by industry.

\section{Risk of bias}

Bias Authors' judgement Support for judgement

Random sequence genera- Low risk tion (selection bias)
Quote: "randomised...in a 1:1 ratio, according to a computer-generated list by block randomisation"

Comment: Specific randomisation method that is suitable was specified

\begin{tabular}{|c|c|c|}
\hline \multirow[t]{2}{*}{$\begin{array}{l}\text { Allocation concealment } \\
\text { (selection bias) }\end{array}$} & Low risk & $\begin{array}{l}\text { Quote: "The study coordinator kept one set of sealed envelopes containing the } \\
\text { treatment codes" }\end{array}$ \\
\hline & & $\begin{array}{l}\text { Comment: Treatment allocation was likely concealed as a centralised co-ordi- } \\
\text { nator controlled the randomisation table }\end{array}$ \\
\hline $\begin{array}{l}\text { Blinding of participants } \\
\text { and personnel (perfor- } \\
\text { mance bias) }\end{array}$ & Low risk & $\begin{array}{l}\text { Quote: "All patients and the investigator involved in conducting the study were } \\
\text { blinded to treatment codes." }\end{array}$ \\
\hline
\end{tabular}


Parekh 2017 (Continued)

All outcomes

Blinding of outcome as- Low risk
sessment (detection bias) All outcomes
Comment: It is likely that blinding was maintained

Comment: The investigator assessed clinical endpoints and was blinded to treatment codes. It is likely that blinding was maintained

\begin{tabular}{lll}
\hline $\begin{array}{l}\text { Incomplete outcome data } \\
\text { (attrition bias) } \\
\text { All outcomes }\end{array}$ & Low risk & $\begin{array}{l}\text { Comment: No participants withdrew from the study. The ITT population was } \\
\text { clearly specified }\end{array}$ \\
\hline $\begin{array}{l}\text { Selective reporting (re- } \\
\text { porting bias) }\end{array}$ & High risk & $\begin{array}{l}\text { Comment: Addition of secondary outcome in the publication that was not in- } \\
\text { cluded in the trial registry "IGA response". CTRI/2012/03/002522 }\end{array}$ \\
\hline Other bias & Low risk & $\begin{array}{l}\text { Comment: Not a multiple intervention trial, with all outcomes reported for in- } \\
\text { terventions }\end{array}$ \\
\hline
\end{tabular}

Paul 2013

$\begin{array}{ll}\text { Methods } & \text { RCT (parallel) } \\ \text { Study design: Open } & \text { Sample size calculation: Yes } \\ & \text { Diagnosis mycology: Culture } \\ & \text { Big toenail specified: Yes } \\ & \text { Statistical comparisons for outcomes: Yes } \\ & \text { Duration of follow-up: Unknown } \\ & \text { Time points of measurements: Weeks 3, 11, 32, and 48 } \\ \text { Location: } 17 \text { centres in France and Tunisia (cities/institutions unknown) }\end{array}$

Participants

Total n: 142

Age: Mean \pm SD (range) amorolfine (AMO) $47.1 \pm 15.2$ years (20 - 84), sequential treatment (SEQ) $47.1 \pm$ 13.8 years $(21-76)$, total $47.1 \pm 14.4$ years $(20-84)$

Sex: AMO: men 32/71 (45.1\%), women 39/71 (54.9\%). SEQ men 32/71 (45.1\%), women 39/71 (54.9\%). Total men 64/142 (45.1\%), women 78/142 (54.9\%)

Inclusion criteria: Aged 18 - 70 years with distal-lateral or lateral subungual onychomycosis caused by T. rubrum were included. They had to have onychomycosis affecting at least 1 big toenail without matrix involvement and showing between $25 \%$ and $60 \%$ of clinically infected nail area and with at least 2 $\mathrm{mm}$ of unaffected proximal target nail area. Eligible participants were required to have a dermatophyte identified by mycological culture. Systemic or topical antifungal therapy had to be stopped at least 3 months before randomisation

Exclusion criteria: People with $>3$ affected nails; people with psoriasis, lichen planus or other abnormalities that could result in clinically abnormal toenail(s); people with moccasin-type tinea pedis, and those with known sensitivity to study treatments. Women were excluded if they were pregnant, breastfeeding or planning a pregnancy during the course of the study

Disease duration: Unknown

Comparable at baseline: The baseline demographic characteristics and mycological results were similar between groups at inclusion 
Paul 2013 (Continued)

Causative species: Onychomycosis caused by T. rubrum were included

Number of people lost to follow-up; AMO $(n=19)$ : Randomisation error $(n=1)$, unauthorised concomitant treatment $(n=2)$, non-respect of period between visits $(n=10)$, observance issues $(n=8)$, non-assessment of the main efficacy criterion $(n=5)$. SEQ $(n=10)$ : non-respect of period between visits $(n=4)$, observance issues $(n=5)$, non-assessment of the main efficacy criterion $(n=2)$. It was possible for more than 1 reason to be attributed to each participant

Trial duration: August 2009 - August 2011

Treatment duration: 36 weeks
Drug in study arm 1: Sequential (SEQ) treatment with chemical nail avulsion with RV4104A ointment for
3 weeks followed by ciclopirox cream for 8 weeks and ciclopirox nail lacquer for 25 weeks (SEQ group),
$\mathrm{n}=71$
Drug in study arm 2: Amorolfine nail lacquer (twice weekly for 36 weeks, AMO), $\mathrm{n}=71$

\begin{tabular}{ll} 
Outcomes & Mycological cure (KOH and culture) \\
Complete cure (the combination of clinical cure and negative mycology) at weeks 3, 11, 32, and 48 \\
Clinical cure (disappearance of all lesions on each nail or residual disease of no more than 10\% of the \\
original total diseased surface) at weeks 3, 11, 32, and 48 \\
Adverse events: reported \\
\hline Notes \\
$\begin{array}{l}\text { s of } 16 \text { authors are employees of industry, while the remaining authors do not state the presence or ab- } \\
\text { sence of conflicts. The study was sponsored by industry }\end{array}$
\end{tabular}

\section{Risk of bias}

\begin{tabular}{lll}
\hline Bias & Authors' judgement & Support for judgement \\
\hline $\begin{array}{l}\text { Random sequence genera- } \\
\text { tion (selection bias) }\end{array}$ & Low risk & $\begin{array}{l}\text { Email response: "A centralized randomisation table (block of 2) was estab- } \\
\text { lished by the Clinical Pharmacy Department...Patients were randomly as- } \\
\text { signed to one of the 2 parallel treatment arms" } \\
\text { Comment: Specific randomisation method that is suitable was specified }\end{array}$ \\
\hline $\begin{array}{l}\text { Allocation concealment } \\
\text { (selection bias) }\end{array}$ & Low risk & $\begin{array}{l}\text { Quote: "The treatment was allocated by means of sequentially numbered con- } \\
\text { tainers to each patient by the investigator, according to the randomisation } \\
\text { list." }\end{array}$ \\
& $\begin{array}{l}\text { Comment: Treatment allocation was likely concealed as a third party con- } \\
\text { trolled randomisation table }\end{array}$
\end{tabular}

\begin{tabular}{|c|c|c|}
\hline $\begin{array}{l}\text { Blinding of participants } \\
\text { and personnel (perfor- } \\
\text { mance bias) } \\
\text { All outcomes }\end{array}$ & High risk & $\begin{array}{l}\text { Quote: "Investigator and patient were not blinded to treatment assignment." } \\
\text { Comment: Participants and personnel were not blinded }\end{array}$ \\
\hline
\end{tabular}

Blinding of outcome as-
sessment (detection bias)

Quote: "Identification of dermatophytes...by two independent and experienced operators who were blinded with respect to the assigned treatment"

All outcomes
Comment: Mycological cure was evaluator-blinded. Clinical outcomes were not evaluator-blinded

Incomplete outcome data Low risk (attrition bias)

All outcomes
Comment: Reasons for participants discontinuing the study were provided for each treatment group. The ITT population was clearly specified 
Paul 2013 (Continued)

Selective reporting (re- High risk Comment: Primary outcome not defined in protocol ("efficacy"), with outporting bias) comes defined in publication. Secondary outcomes identified in the protocol are reported in the publication. NCT01014637

Other bias Low risk Comment: Not a multiple intervention trial; all outcomes reported for inter-
ventions

\section{Romero-Cerecero 2008}

\begin{tabular}{ll}
\hline Methods & RCT (parallel) \\
Study design: Double blind \\
Sample size calculations: No \\
Diagnosis mycology: Direct examination \\
Big toenail specified: No \\
Statistical comparison for outcomes: Yes \\
Duration of follow-up: Unknown \\
Time points of measurements: Months 1, 2 and 3 \\
Location: unknown
\end{tabular}

\section{Participants}

Total n: 110

Age: Mean \pm SD (range): A. pichinchensis $44.6 \pm 11.9$ years, ciclopirox $46.5 \pm 12.2$ years, (18-56).

Sex: A. pichinchensis: men 14/55 (25.4\%), women 41/55 (74.5\%). Ciclopirox: men 18/55 (32.7\%), women $37 / 55(67.2 \%)$

Inclusion criteria: People with tinea pedis, with diagnosis of diabetes, as well as pregnant woman or women who were breast-feeding, or patients who had received treatment for onychomycosis 1 month prior to study initiation, and those allergic to ciclopirox, were not included

Exclusion criteria: Noncompliance with the treatment was identified or due to the presence of intense side effects

Disease duration: Disease evolution $\leq 1$ year A. pich 20/55 (36.3\%) ciclopirox $13 / 55$ (23.6\%) 2 - 5 years A. pich $23 / 55(41.8 \%)$ ciclopirox $22 / 55(40.1 \%)>5$ years A. pich $5 / 55(9.2 \%)$ ciclopirox $8 / 55(14.5 \%) \geq 10$ years A. pich 7/55 (12.7\%) ciclopirox 12/55 (21.8\%)

Comparable at baseline: Personal characteristics (concerning family and disease) of participants did not show differences between the groups Statistical analysis did not evidence inter-group differences; under basal conditions, we observed that only nail colour alteration was higher in the experimental group

Causative species: Most frequently isolated pathogen agent (80\%) was T. rubrum

Number of people lost to follow-up: Experimental $n=6$, control $n=8$

Trial duration: unknown

Drug in study arm 1: A. pichinchensis extract (10\%), $n=55$ 
Romero-Cerecero 2008 (Continued)

Drug in study arm 2: 8\% ciclopirox (control), $\mathrm{n}=55$

\begin{tabular}{|c|c|c|}
\hline \multirow[t]{3}{*}{ Outcomes } & \multicolumn{2}{|c|}{ Mycological effectiveness ( $\mathrm{KOH}$ microscopy) at months 1,2 and 3} \\
\hline & \multicolumn{2}{|c|}{ Clinical effectiveness (signs or symptoms were found on affected nails) at months 1,2 and 3} \\
\hline & \multicolumn{2}{|c|}{ Adverse events: reported } \\
\hline Notes & \multicolumn{2}{|c|}{$\begin{array}{l}\text { Public funding for study. Authors do not state presence or absence of conflicts of interest and are affili- } \\
\text { ated with academic and public research institutions }\end{array}$} \\
\hline \multicolumn{3}{|l|}{ Risk of bias } \\
\hline Bias & Authors' judgement & Support for judgement \\
\hline \multirow[t]{2}{*}{$\begin{array}{l}\text { Random sequence genera- } \\
\text { tion (selection bias) }\end{array}$} & Low risk & $\begin{array}{l}\text { Quote: "Experimental and control treatments were distributed (in agreement } \\
\text { with a table with randomised numbers) to patients consenting..." }\end{array}$ \\
\hline & & Comment: Specific randomisation method that is suitable was specified \\
\hline $\begin{array}{l}\text { Allocation concealment } \\
\text { (selection bias) }\end{array}$ & Unclear risk & $\begin{array}{l}\text { Comment: The party that carried out randomisation and treatment allotment } \\
\text { is not explicitly stated in the article }\end{array}$ \\
\hline $\begin{array}{l}\text { Blinding of participants } \\
\text { and personnel (perfor- } \\
\text { mance bias) }\end{array}$ & Low risk & $\begin{array}{l}\text { Quote: "the flask containing experimental treatment had a similar presenta- } \\
\text { tion to that of the control treatment", "the control group received an identical } \\
\text { flask" }\end{array}$ \\
\hline \multicolumn{3}{|r|}{ Comment: Blinding was adequate } \\
\hline $\begin{array}{l}\text { Blinding of outcome as- } \\
\text { sessment (detection bias) } \\
\text { All outcomes }\end{array}$ & Unclear risk & $\begin{array}{l}\text { Comment: Blinded, but no specific mention of outcome assessor blinding } \\
\text { method }\end{array}$ \\
\hline $\begin{array}{l}\text { Incomplete outcome data } \\
\text { (attrition bias) } \\
\text { All outcomes }\end{array}$ & Low risk & $\begin{array}{l}\text { Comment: Reasons for patients discontinuing the study were provided for } \\
\text { each treatment group. The ITT population was clearly specified }\end{array}$ \\
\hline $\begin{array}{l}\text { Selective reporting (re- } \\
\text { porting bias) }\end{array}$ & Unclear risk & $\begin{array}{l}\text { Comment: Review authors were unable to find a clinical trial register entry for } \\
\text { this study }\end{array}$ \\
\hline Other bias & Low risk & $\begin{array}{l}\text { Comment: Not a multiple intervention trial, all outcomes reported for inter- } \\
\text { ventions }\end{array}$ \\
\hline
\end{tabular}

\section{Romero-Cerecero 2009}

\begin{tabular}{ll} 
Methods & RCT (parallel) \\
Study design: Double blind \\
Sample size calculation: No \\
Diagnosis mycology: $\mathrm{KOH}$ \\
Big toenail specified: $\mathrm{No}$ \\
Statistical comparisons to outcomes: Yes \\
Duration of follow-up: Unknown \\
Time points of measurements: Months $1,2,3,4,5$ and 6 \\
\hline
\end{tabular}


Romero-Cerecero 2009 (Continued)

Location: Mexico (city/institution unknown)

Participants
Age: Median (range): 47 years, (19 and 65$).$
Sex: Men 28/122 (23\%), women $94 / 122(77 \%)$
Inclusion criteria: A diagnosis of mild or moderate onychomycosis aged $19-65$ years
Exclusion criteria: Pregnant or breastfeeding, with diabetes mellitus or another serious disease, as well
as people who received treatment for this disease at least 1 month prior to the date of study inclusion,
or with a history of sensitivity to topically administrated drugs
Disease duration: Unknown
Comparable at baseline: Distribution of these variables between both groups was similar, and no signif-
icant differences were detected
Causative species: Unknown
Number of people lost to follow-up: Group $1 \mathrm{n}=7$, group $2 \mathrm{n}=12$
Trial duration: unknown
Treatment duration: 6 months
Drug in study arm $1: 12.6 \%$ Ageratina pichinchensis standardised extract (in a nail lacquer solution), $\mathrm{n}=$
62
Drug in study arm 2: $16.8 \%$ Ageratina pichinchensis standardised extract (in a nail lacquer solution), $\mathrm{n}=$
60

60

Outcomes Mycological diagnosis (microscopy) at months 1, 2, 3, 4, 5 and 6

Adverse events: reported

Notes

Public funding for the study. Authors do not state presence of absence of conflicts of interest and are affiliated with academic and public research institutions

\section{Risk of bias}

\begin{tabular}{|c|c|c|}
\hline Bias & Authors' judgement & Support for judgement \\
\hline \multirow[t]{2}{*}{$\begin{array}{l}\text { Random sequence genera- } \\
\text { tion (selection bias) }\end{array}$} & Low risk & $\begin{array}{l}\text { Quote: "Based on a table with randomised numbers, two groups were orga- } \\
\text { nized" }\end{array}$ \\
\hline & & Comment: Specific randomisation method that is suitable was specified \\
\hline $\begin{array}{l}\text { Allocation concealment } \\
\text { (selection bias) }\end{array}$ & Unclear risk & $\begin{array}{l}\text { Comment: The party that carried out randomisation and treatment allotment } \\
\text { is not explicitly stated in the article }\end{array}$ \\
\hline \multirow{2}{*}{$\begin{array}{l}\text { Blinding of participants } \\
\text { and personnel (perfor- } \\
\text { mance bias) } \\
\text { All outcomes }\end{array}$} & Low risk & $\begin{array}{l}\text { Quote: "the medicaments (in a 3-mL flask presentation with a spiral cover that } \\
\text { contained a brush for administration on nails) were packed and labelled in } \\
\text { identical form" }\end{array}$ \\
\hline & & Comment: Blinding was adequate \\
\hline
\end{tabular}

Blinding of outcome as- Unclear risk sessment (detection bias)

All outcomes
Comment: Blinded, but no specific mention of outcome assessor blinding method 
Romero-Cerecero 2009 (Continued)

$\begin{array}{ll}\begin{array}{l}\text { Incomplete outcome data Low risk } \\ \text { (attrition bias) }\end{array} & \begin{array}{l}\text { Comment: Reasons for participants discontinuing the study were provided for } \\ \text { each treatment group. The ITT population was clearly specified }\end{array}\end{array}$

All outcomes

\begin{tabular}{lll}
$\begin{array}{l}\text { Selective reporting (re- } \\
\text { porting bias) }\end{array}$ & Unclear risk & $\begin{array}{l}\text { Comment: Review authors were unable to find a clinical trial register entry for } \\
\text { this study }\end{array}$ \\
\hline Other bias & Low risk & $\begin{array}{l}\text { Comment: Not a multiple intervention trial, and all outcomes reported for in- } \\
\text { terventions }\end{array}$
\end{tabular}

\section{Schalka 2012}

Methods $\quad$ RCT (parallel)
Study design: Single blind
Sample size calculation: No
Diagnosis mycology: $\mathrm{KOH}$ and culture
Big toe specified: Yes
Statistical comparisons for outcomes: Yes
Duration of follow-up: At 5 and 180 days
Time points of measurements: Days 60, 120 and 180
Location: unknown

\section{Participants}

Total n: 41

Age: Average (range): Group 141.2 years (18 - 63), Group 241.2 years (19 - 65)

Sex: Group 1: men 5/20 (25\%), women 15/20 (75\%). Group 2: men 1/21 (5\%), women 20/21 (95\%)

Inclusion criteria: Men and women, 18 - 65 years of age with distal or lateral subungual onychomycosis of the first right or left toe, between $10 \%-50 \%$ involvement of the nail plate and without matrix involvement

\section{Exclusion criteria: Unknown}

Disease duration: Average, median \pm SD (range): Group 1: 22.3 months, $12 \pm 26.7$ months (6 - 120); Group 2: 18.2 months, $12 \pm 17.5$ months ( 3 - 84)

Comparable at baseline: The presence of fungus on the baseline evaluation was similar in both treatment groups. When analysing the results in terms of the population evaluated we noticed a homogeneous distribution between the 2 groups, in terms of sex, age, involved area, proportion of affected nail, time of evolution and previous treatments, this way reducing the interference of these characteristics on the interpretation of the results

Causative species: Predominance of the Trichophyton genus, the Trichophyton rubrum and Trichophyton mentagrophytes being represented, respectively, in $55.0 \%$ and $30 \%$ of the people that used the posology of once a week (group I), and in $61.9 \%$ and $19 \%$ of those of the $3 / 2 / 1$ regimen (Group 2). The other species identified were Scytalidium dimidiatum and Candida albicans with a frequency of $<10 \%$ Number of participants lost to follow-up: 0

Trial duration: unknown

Interventions Drug in study arm 1: Ciclopirox nail lacquer daily for 6 months, $\mathrm{n}=20$


Schalka 2012 (Continued)

Drug in study arm 2: Ciclopirox nail lacquer 3/2/1 (3x a week in the first month, twice weekly for the second month and weekly till the end of the sixth month), $n=21$

\begin{tabular}{|c|c|c|}
\hline \multirow[t]{4}{*}{ Outcomes } & \multicolumn{2}{|c|}{ Mycological cure (negative culture) at days 60, 120 and 180} \\
\hline & \multicolumn{2}{|c|}{$\begin{array}{l}\text { Therapeutic success (negative culture and clinical improvement }>90 \% \text { of the healthy nail) at days } 60 \text {, } \\
120 \text { and } 180\end{array}$} \\
\hline & \multicolumn{2}{|c|}{$\begin{array}{l}\text { Clinical improvement (negative culture and improvement between } 50 \% \text { and } 90 \% \text { of the affected nail } \\
\text { and the percentage of improvement of the extension of the affected nail) at days } 60,120 \text { and } 180\end{array}$} \\
\hline & \multicolumn{2}{|c|}{ Adverse events: reported } \\
\hline Notes & \multicolumn{2}{|c|}{$\begin{array}{l}\text { All } 3 \text { authors have ties to industry (e.g. consultant, speaker). Grant for performing the study were re- } \\
\text { ceived from industry }\end{array}$} \\
\hline \multicolumn{3}{|l|}{ Risk of bias } \\
\hline Bias & Authors' judgement & Support for judgement \\
\hline \multirow{2}{*}{$\begin{array}{l}\text { Random sequence genera- } \\
\text { tion (selection bias) }\end{array}$} & \multirow[t]{2}{*}{ Unclear risk } & Quote: "randomly assigned" \\
\hline & & Comment: Method of randomisation not specified \\
\hline $\begin{array}{l}\text { Allocation concealment } \\
\text { (selection bias) }\end{array}$ & Unclear risk & Comment: No information was available \\
\hline $\begin{array}{l}\text { Blinding of participants } \\
\text { and personnel (perfor- } \\
\text { mance bias) } \\
\text { All outcomes }\end{array}$ & High risk & $\begin{array}{l}\text { Comment: This is a single-blind study comparing } 2 \text { different regimens of the } \\
\text { same therapeutic agent. Participants were aware of treatment }\end{array}$ \\
\hline $\begin{array}{l}\text { Blinding of outcome as- } \\
\text { sessment (detection bias) } \\
\text { All outcomes }\end{array}$ & Unclear risk & $\begin{array}{l}\text { Comment: It is possible that outcome assessors were blinded (single-blind } \\
\text { study). This is not explicitly stated in the article }\end{array}$ \\
\hline $\begin{array}{l}\text { Incomplete outcome data } \\
\text { (attrition bias) } \\
\text { All outcomes }\end{array}$ & Low risk & Comment: All participants completed the study \\
\hline $\begin{array}{l}\text { Selective reporting (re- } \\
\text { porting bias) }\end{array}$ & Unclear risk & $\begin{array}{l}\text { Comment: Review authors were unable to find a clinical trial register entry for } \\
\text { this study }\end{array}$ \\
\hline Other bias & Low risk & $\begin{array}{l}\text { Comment: Not a multiple intervention trial, with all outcomes reported for in- } \\
\text { terventions }\end{array}$ \\
\hline
\end{tabular}

\section{Sigurgeirsson 2016}

\begin{tabular}{ll} 
Methods & RCT (parallel) \\
Study design: Single blind \\
Sample size calculator: $\mathrm{No}$ \\
Diagnosis mycology: $\mathrm{KOH}$ and culture \\
Big toenail specified: Yes \\
Statistical comparisons to outcomes: Yes \\
\hline
\end{tabular}


Sigurgeirsson 2016 (Continued)

Duration of follow-up: At 7 days post-final treatment

Time points of measurements: Week 12

Location: Reykjavik, Iceland (institution name unknown)

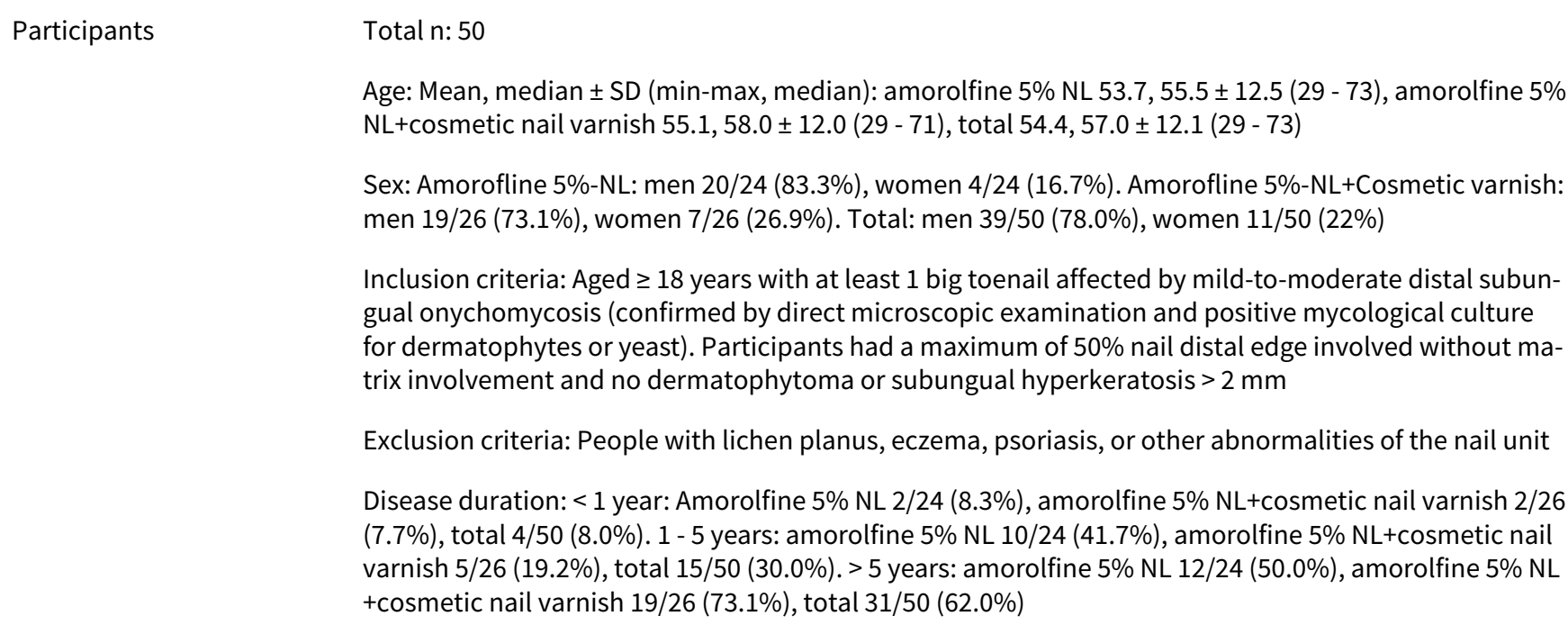

Comparable at baseline: Both groups were comparable in terms of dermographic characteristics

Causative Species: Dermatophytes or yeast

Number of people lost to follow-up: $n=1$ in each group

Trial duration: February 2014 - January 2016

$\begin{array}{ll}\text { Interventions } & \text { Treatment duration: once weekly for } 12 \text { weeks } \\ & \text { Drug in study arm 1: Amorolfine } 5 \% \mathrm{NL} \text { (alone), } \mathrm{n}=24 \\ & \text { Drug in study arm 2: Amorolfine } 5 \% \mathrm{NL}+\text { a cosmetic nail varnish (applied } 24 \text { hours later), } \mathrm{n}=26\end{array}$

Outcomes Mycological cure (culture) at week 12

Adverse events: reported

Notes

3 of 5 authors are employees of industry. The remaining 2 authors received payment to conduct the study. The study was sponsored by industry

\section{Risk of bias}

Bias Authors' judgement Support for judgement

Random sequence genera- Low risk tion (selection bias)
Quote: "A randomisation list was generated using the Ranuni routine of the statistical analysis system and a kit number was assigned."

Comment: Specific randomisation method that is suitable was specified

\begin{tabular}{lll}
\hline $\begin{array}{l}\text { Allocation concealment } \\
\text { (selection bias) }\end{array}$ & Unclear risk & Comment: No information on how allocation was concealed \\
\hline $\begin{array}{l}\text { Blinding of participants } \\
\begin{array}{l}\text { and personnel (perfor- } \\
\text { mance bias) }\end{array}\end{array}$ & High risk & $\begin{array}{l}\text { Comment: This is an investigator-blinded study. Participants and personnel } \\
\text { were aware of treatment }\end{array}$
\end{tabular}


Sigurgeirsson 2016 (Continued)

All outcomes

\section{Blinding of outcome as- Low risk} sessment (detection bias) All outcomes
Quote: "To maintain blinding, the person dispensing the study drug and the subject were instructed not to discuss the study treatments with the evaluating investigator."

Comment: It is likely that investigators remained blinded

\begin{tabular}{lll}
$\begin{array}{l}\text { Incomplete outcome data } \\
\text { (attrition bias) } \\
\text { All outcomes }\end{array}$ & Low risk & $\begin{array}{l}\text { Comment: Reasons for participants discontinuing the study were provided for } \\
\text { each treatment group. The ITT population was clearly specified }\end{array}$ \\
\hline $\begin{array}{l}\text { Selective reporting (re- } \\
\text { porting bias) }\end{array}$ & Low risk & $\begin{array}{l}\text { Comment: Efficacy outcomes stated in trial protocol reported in publication. } \\
\text { NCT02321098 }\end{array}$ \\
\hline Other bias & Low risk & $\begin{array}{l}\text { Comment: Not a multiple intervention trial, with all outcomes reported for in- } \\
\text { terventions }\end{array}$
\end{tabular}

Stein 2014

\begin{tabular}{|c|c|}
\hline \multirow[t]{9}{*}{ Methods } & RCT (parallel) \\
\hline & Study design: Double blind \\
\hline & Sample size calculation: Unknown \\
\hline & Diagnosis mycology: $\mathrm{KOH}$ and culture \\
\hline & Big toenail specified: Yes \\
\hline & Statistical comparisons for outcomes: Unknown \\
\hline & Duration of follow-up: 4 weeks \\
\hline & Time points of measurements: Week 52 \\
\hline & Location: United States (city/institution unknown) \\
\hline \multirow[t]{9}{*}{ Participants } & Total n: 334 \\
\hline & Age: 18 - 70 years of age, inclusive \\
\hline & Sex: Men and women (ratio unknown) \\
\hline & $\begin{array}{l}\text { Inclusion criteria: Adult men and women, any race, } 18-70 \text { years of age with a clinical diagnosis of mild- } \\
\text { to-moderate distal subungual onychomycosis, } 25 \%-50 \% \text { nail involvement and positive } \mathrm{KOH} \text { and cul- } \\
\text { ture. Participants had to be free of disease that in the investigator's opinion might interfere with study } \\
\text { evaluations or jeopardise patient safety }\end{array}$ \\
\hline & $\begin{array}{l}\text { Exclusion criteria: History of intolerance or hypersensitivity to imidazole compounds or the inactive } \\
\text { components of the solution. Current or recent participation in another investigational medication or } \\
\text { device study }\end{array}$ \\
\hline & Disease duration: Unknown \\
\hline & Comparable at baseline: Unknown \\
\hline & Causative species: Unknown \\
\hline & Number of participants lost to follow-up: Unknown \\
\hline
\end{tabular}


Stein 2014 (Continued)

Trial duration: unknown

\begin{tabular}{ll}
\hline Outcomes & Complete cure at week 52 \\
& Adverse events: reported \\
\hline Notes & $\begin{array}{l}\text { Conference abstract of interim results so demographics limited. } 1 \text { of } 6 \text { authors has industry affiliation. } \\
\text { Study supported by industry }\end{array}$ \\
\hline
\end{tabular}

\section{Risk of bias}

\begin{tabular}{|c|c|c|}
\hline Bias & Authors' judgement & Support for judgement \\
\hline \multirow{2}{*}{$\begin{array}{l}\text { Random sequence genera- } \\
\text { tion (selection bias) }\end{array}$} & \multirow[t]{2}{*}{ Unclear risk } & Quote: "patients have been randomised" \\
\hline & & Comment: Method of randomisation not specified \\
\hline $\begin{array}{l}\text { Allocation concealment } \\
\text { (selection bias) }\end{array}$ & Unclear risk & Comment: No information was available \\
\hline $\begin{array}{l}\text { Blinding of participants } \\
\text { and personnel (perfor- } \\
\text { mance bias) } \\
\text { All outcomes }\end{array}$ & Unclear risk & Comment: No information was available \\
\hline $\begin{array}{l}\text { Blinding of outcome as- } \\
\text { sessment (detection bias) } \\
\text { All outcomes }\end{array}$ & Unclear risk & Comment: No information was available \\
\hline $\begin{array}{l}\text { Incomplete outcome data } \\
\text { (attrition bias) } \\
\text { All outcomes }\end{array}$ & Unclear risk & Comment: No information was available \\
\hline $\begin{array}{l}\text { Selective reporting (re- } \\
\text { porting bias) }\end{array}$ & Low risk & $\begin{array}{l}\text { Comment: Efficacy outcomes stated in trial protocol also stated in abstract. } \\
\text { NCT01431820 }\end{array}$ \\
\hline Other bias & Unclear risk & $\begin{array}{l}\text { Comment: Multiple treatment arms, but interim abstract of study's progress } \\
\text { and no results to assess }\end{array}$ \\
\hline
\end{tabular}

Syed 1998

$\begin{array}{ll}\text { Methods } & \text { RCT (parallel) } \\ & \text { Study design: Double blind } \\ & \text { Sample size calculation: } \mathrm{No} \\ & \text { Diagnosis mycology: } \mathrm{KOH} \text { and culture }\end{array}$


Syed 1998 (Continued)

Big toenail specified: Yes

Statistical comparisons for outcomes: Yes

Duration of follow-up: At 16 and 36 weeks

Time points of measurements: Weeks 8,16 and 24

Location: unknown

\section{Participants}

Total n: 60

Age: Mean: treatment 27.5, control 26.9, (18 - 60)

Sex: Treatment: men 32/50 (64\%), women 18/50 (36\%), control: men 6/10 (60\%), women 4/10 (40\%)

Inclusion criteria: Fungal infections on at least 1 big toenail with more than $25 \%$ involvement of the entire nail bed

Exclusion criteria: Included onychomycosis caused by moulds, bacteria, and/or Candida spp., absence of a dermatophyte, a history or presence of psoriasis, any serious concurrent disease, hypersensitivity to azole or benzylamine derivatives, or concomitant therapy with drugs that might affect the bioavailability of benzylamines. People were also excluded if they had used any topical antifungal compound within 2 weeks, had received any systemic antifungal therapy $<3$ months before the enrolment, or were pregnant or nursing mothers

Disease duration: 4 to 24 months.

Comparable at baseline: Statistically, participants who received active or placebo preparations were similar in age, race, number, and area of toenail infection

Causative species; Active cream: T. rubrum 47/50 (94\%), T. tonsurans 2/50 (4\%), T. mentagrophytes 1/50 (2\%). Placebo: T. rubrum $7 / 10$ (70\%),

T. tonsurans $1 / 10(10 \%)$, T. mentagrophytes $2 / 10(20 \%)$

Number of participants lost to follow-up: None

Trial duration: unknown

Drug in study arm 1: Butenafine hydrochloride (2\%) and urea (20\%), $n=50$

Drug in study arm 2: Placebo, $\mathrm{n}=10$

\section{Outcomes}

Complete cure (negative fungal culture on fresh specimen, no growth of dermatophytes in culture medium, progressive growth of normal nail and resolution of all clinical symptoms) at weeks 8,16 and 24

Adverse events: reported conflicts of interest are provided

\section{Risk of bias}

\section{Bias}

Authors' judgement Support for judgement

Random sequence genera- Unclear risk tion (selection bias)
Quote: "randomly allocated"

Comment: Method of randomisation not specified 
Syed 1998 (Continued)

Allocation concealment Unclear risk Comment: No information was available
(selection bias)

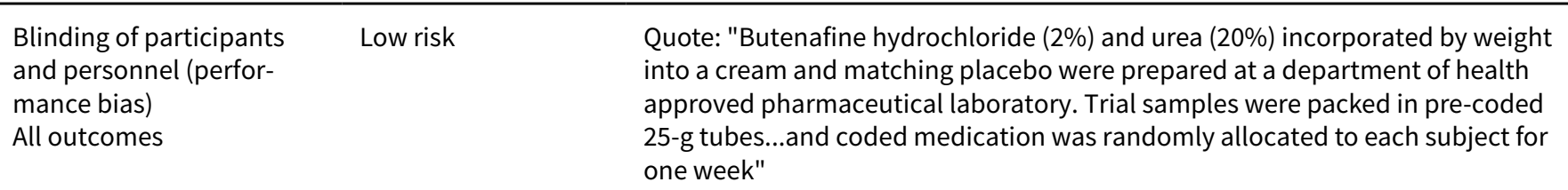

Comment: Blinding was adequate

\begin{tabular}{lll}
\hline $\begin{array}{l}\text { Blinding of outcome as- } \\
\text { sessment (detection bias) }\end{array} \quad$ Unclear risk & $\begin{array}{l}\text { Comment: Blinded, but no specific mention of outcome assessor blinding } \\
\text { method }\end{array}$
\end{tabular}

All outcomes

\begin{tabular}{lll}
\hline $\begin{array}{l}\text { Incomplete outcome data } \\
\text { (attrition bias) } \\
\text { All outcomes }\end{array}$ & Low risk & Comment: All participants who were enrolled completed the study \\
\hline $\begin{array}{l}\text { Selective reporting (re- } \\
\text { porting bias) }\end{array}$ & Low risk & $\begin{array}{l}\text { Comment: Outcomes specified in Method reported in Results. Study pre-dates } \\
\text { trial registry in the USA }\end{array}$ \\
\hline Other bias & Low risk & $\begin{array}{l}\text { Comment: Multiple intervention trial, but outcomes for all interventions re- } \\
\text { ported separately }\end{array}$ \\
\hline
\end{tabular}

\section{Syed 1999}

\begin{tabular}{|c|c|}
\hline \multirow[t]{9}{*}{ Methods } & $\mathrm{RCT}$ (parallel) \\
\hline & Study design: Double blind \\
\hline & Sample size calculation: No \\
\hline & Diagnosis mycology: $\mathrm{KOH}$ and culture \\
\hline & Big toenail specified: Yes \\
\hline & Statistical comparisons for outcomes: Yes \\
\hline & Duration of follow-up: At 36 weeks \\
\hline & Time points of measurements: Weeks 8, 24 and 36 \\
\hline & Location: unknown \\
\hline \multirow[t]{5}{*}{ Participants } & Total $\mathrm{n}: 60$ \\
\hline & Age: Mean (range): active 29.6, placebo 29.7, total 29.6, (18 - 80) \\
\hline & $\begin{array}{l}\text { Sex: Active: men 24/40 (60\%), female 16/40 (40\%). Placebo: men 15/20 (75\%), women 5/20 (25\%). Total: } \\
\text { men 31/60 (52\%), women 29/60 (48\%) }\end{array}$ \\
\hline & $\begin{array}{l}\text { Inclusion criteria: Aged } 18-80 \text { years (mean } 29.6 \text { ) with at least } 25 \% \text { involvement of fungal infection of } 1 \\
\text { of the large toenails and clinical diagnosis of distal subungual onychomycosis (confirmed mycological- } \\
\text { ly by } 30 \% \mathrm{KOH} \text { wet mount and positive culture for dermatophytes) entered the study }\end{array}$ \\
\hline & $\begin{array}{l}\text { Exclusion criteria: Screened at baseline, were onychomycosis caused by moulds, bacteria or Candida } \\
\text { spp., a history of psoriasis, any serious concurrent disease, hypersensitivity to Melaleuca alternifolia oil, } \\
\text { azole or benzylamine derivatives and concomitant therapy with drugs appearing to affect the bioavail- } \\
\text { ability of benzylamines. Another exclusion criterion was absence of dermatophyte; thus, people who }\end{array}$ \\
\hline
\end{tabular}


Syed 1999 (Continued)

had received systemic antifungal treatment within the previous 3 months or who were using topical treatment during the 2 weeks preceding the study period were not enrolled. Pregnant and lactating women were not recruited

Disease duration: Active: 14.3 months, placebo: 15.0 months, total: 6 - 36 months. 29 participants

$(48.3 \%)$ had suffered from nail disease for $<1$ year; $51.7 \%$ for $1-3$ years

Comparable at baseline: Statistically both groups were comparable in age, race, number and area of toenail infection

Causative species: T. rubrum: active 38/40 (95\%), placebo 18/20 (90\%).T. tonsurans, active 1/40 (2.5\%), placebo $2 / 20$ (10\%). T. mentagrophytes: active $1 / 40(2.5 \%)$, placebo $0 / 20(0 \%)$

Number of people lost to follow-up: None

Trial duration: unknown

Treatment duration: 8 weeks max, 3 times a day, with occlusive dressing
Drug in study arm 1: $2 \%$ butenafine hydrochloride and 5\% Melaleuca alternifolia oil, $\mathrm{n}=40$
Drug in study arm 2: Placebo (containing Melaleuca alternifolia oil), $\mathrm{n}=20$

\begin{tabular}{ll}
\hline Outcomes & Mycological cure (KOH and culture) \\
& Clinical success (100\% remission or $90 \%$ to $99 \%$ improvement in treated toenail) at weeks 8,24 and 36 \\
& Overall cure (resolution of all clinical symptoms with respect to global assessment together with myco- \\
logical cure and progressive growth of normal nail) at weeks 8,24 and 36 \\
Adverse events: reported
\end{tabular}

\section{Risk of bias}

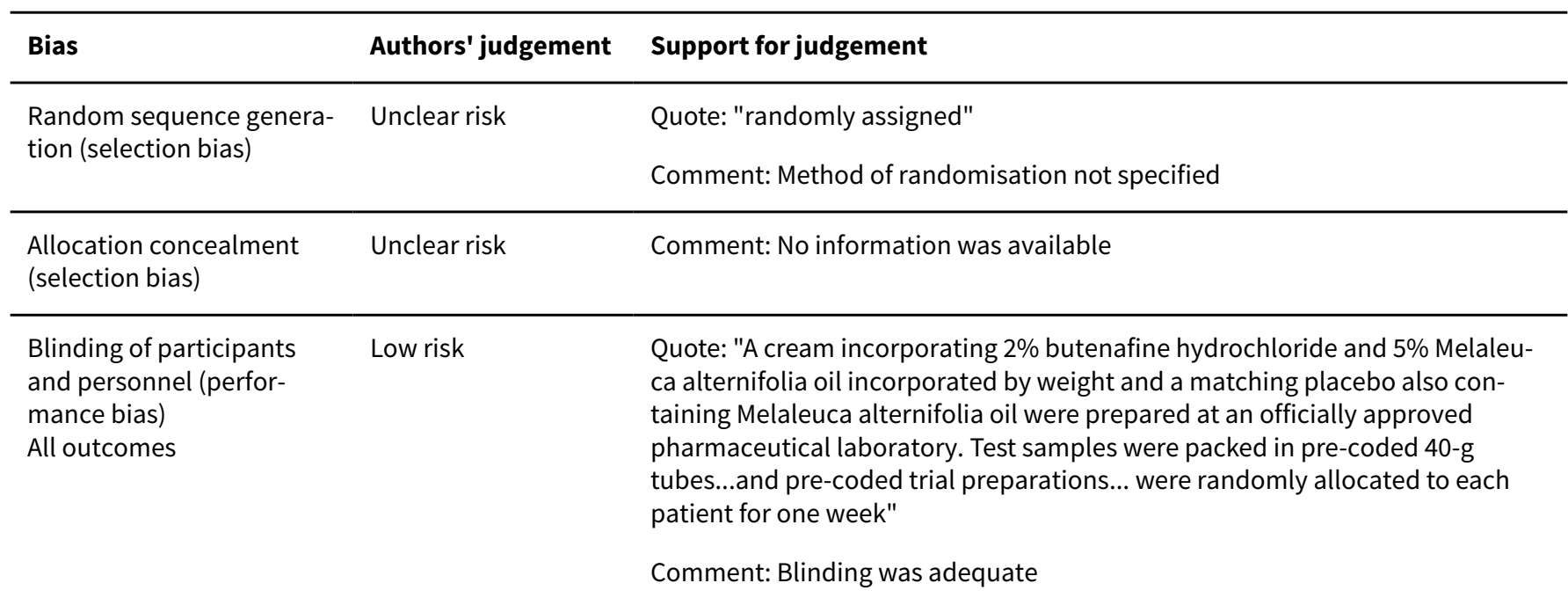

\begin{tabular}{lll}
\hline $\begin{array}{l}\text { Blinding of outcome as- } \\
\text { sessment (detection bias) }\end{array}$ & Unclear risk & $\begin{array}{l}\text { Comment: Blinded, but no specific mention of outcome assessor blinding } \\
\text { method }\end{array}$ \\
All outcomes &
\end{tabular}

Incomplete outcome data Low risk Comment: All participants who were enrolled completed the study
(attrition bias)


Syed 1999 (Continued)

All outcomes

$\begin{array}{ll}\begin{array}{l}\text { Selective reporting (re- } \quad H i g h \text { risk } \\ \text { porting bias) }\end{array} & \begin{array}{l}\text { Comment: Not all efficacy outcomes stated in the Methods were reported in } \\ \text { the Results }\end{array}\end{array}$

\begin{tabular}{ll}
\hline Other bias $\quad$ Low risk & $\begin{array}{l}\text { Comment: Multiple intervention trial, but outcomes for all interventions re- } \\
\text { ported separately }\end{array}$
\end{tabular}

\begin{tabular}{ll}
\hline Methods & RCT (parallel) \\
Study design: Double blind \\
Sample size calculation: No \\
Diagnosis mycology: $\mathrm{KOH}$ and culture \\
Big toenail specified: Yes \\
Statistical comparisons for outcomes: Yes \\
Duration of follow up: At 6 months, efficacy measured at 180 and 360 days \\
Time points of measurement: Days 180 and 360 \\
Location: United States and Mexico (cities/institutions unknown) \\
\hline
\end{tabular}

Participants

Total n: 187

Age: Mean age \pm SD (range): vehicle $43.6 \pm 9.0$ years ( $18-62), 2.5 \%$ tavaborole $44.3 \pm 9.8$ years $(25-62)$, $5.0 \%$ tavaborole $39.6 \pm 12.2$ years $(21-64), 7.5 \%$ tavaborole $43.1 \pm 12.1$ years ( $18-65)$

Sex: Vehicle: men 37/63 (58.7\%), women 26/63 (41.3\%). 2.5\% Tavaborole: men 19/33 (57.6\%), women 14/33 (42.4\%). 5.0\% Tavaborole: men 19/31 (61.3\%), women 12/31 (38.7\%). 7.5\% Tavaborole: men $38 / 60(63.3 \%)$, women $22 / 60(36.7 \%)$

Inclusion criteria: Adults 18 - 65 years of age with onychomycosis involving $20 \%$ - $60 \%$ of at least 1 great toenail based on visual inspection and confirmed by positive $\mathrm{KOH}$ wet mount or positive fungal culture or both for a dermatophyte. The combined thickness of the affected distal nail plate and hyperkeratotic nail bed must have been $<3 \mathrm{~mm}$. Enrolled participants were required to have normal or clinically non-significant laboratory findings at screening

Exclusion criteria: A history of failed topical antifungal therapy for onychomycosis; anatomic abnormalities of the toe or nail; chronic moccasin-type tinea pedis; concurrent lichen planus; and current or historical evidence of psoriasis. Also excluded were individuals with diabetes mellitus requiring treatment other than diet and exercise and those with a history of significant internal disease. Concurrent use of topical anti-inflammatories, topical antifungals applied to feet (except in the case of tinea pedis), topical or systematic antifungals, corticosteroids, and immunomodulators was not permitted. People who had undergone appropriate washout of these medications prior to baseline were permitted to participate. Debridement of the nails was not permissible during the study

Disease duration: Unknown

Comparable at baseline: The treatment groups in each of the studies were demographically similar

Causative species: Dermatophytes

Number of participants lost to follow-up: (by email response) Total $n=119$; vehicle $\mathrm{N}=26$ (lack of efficacy/worsening of condition $n=18$; participant request $n=2$; lost to follow-up $n=6$ ); tavaborole $2.5 \% n$ $=10$ (lack of efficacy/worsening of condition $n=5$; lost to follow-up $n=5)$; tavaborole $5.0 \% n=10$ (lack 
Toledo-Bahena 2014 (Continued)

of efficacy/worsening of condition $n=3$; lost to follow-up $n=7$ ); tavaborole $7.5 \% n=22$ (lack of effica$c y /$ worsening of condition $n=7$; adverse event $n=1$; participant request $n=4$; lost to follow-up $n=9$; death $\mathrm{n}=1$ )

Trial duration: unknown

$\begin{array}{ll}\text { Interventions } & \text { Treatment duration: Once daily for } 3 \text { months; } \\ & \text { Drug in study arm 1: Vehicle, } \mathrm{n}=63 \\ & \text { Drug in study arm 2: } 2.5 \% \text { tavaborole, } \mathrm{n}=33 \\ & \text { Drug in study arm 3:5.0\% tavaborole, } \mathrm{n}=31 \\ & \text { Drug in study arm 4: } 7.5 \% \text { tavaborole, } \mathrm{n}=60\end{array}$

Outcomes

Mycological cure (KOH and culture) at days 180 and 360

Treatment response (success or failure) at days 180 and 360

Mycological response (negative dermatophyte culture) at days 180 and 360

Complete response (ISGA score of clear, negative fungal culture and a negative $\mathrm{KOH}$ and the TGT) at days 180 and 360

Adverse events: reported

Notes

2 of 8 authors are employees of industry, with the remaining 6 authors reporting ties to industry (e.g. consultant, investigator, speaker). Funded by industry. Disclosures accompanied by a quote: "The authors were fully responsible for the content, editorial decisions, and opinions expressed in the current article. No author received an honorarium related to the development of this manuscript."

\section{Risk of bias}

Bias Authors' judgement Support for judgement

Random sequence genera- Low risk tion (selection bias)
Email response: "A computer generated set of random numbers starting with 101 were blocked in groups of 6 ...The randomisation list was securely retained by (the labelling and packaging contractor) and (the) selected clinical data management and biostatistician contractor...After meeting all qualification (baseline) criteria, the subject was assigned to a test agent treatment using the next sequential test agent number."

Comment: Specific randomisation method that is suitable was specified

Allocation concealment Low risk

(selection bias)

Email response: "The randomisation list was securely retained by (the labelling and packaging contractor) and [the] selected clinical data management and biostatistician contractor."

Comment: Allocation concealment was appropriate

\begin{tabular}{|c|c|c|}
\hline $\begin{array}{l}\text { Blinding of participants } \\
\text { and personnel (perfor- } \\
\text { mance bias) } \\
\text { All outcomes }\end{array}$ & Low risk & $\begin{array}{l}\text { Email response: "Subjects, investigators, and research staff were blinded to } \\
\text { the subject treatment assigned during the study." "(Tavaborole) Solution and } \\
\text { Solution Vehicle were packaged in (identical) } 15 \mathrm{~mL} \text { bottles." } \\
\text { Comment: Blinding was adequate }\end{array}$ \\
\hline $\begin{array}{l}\text { Blinding of outcome as- } \\
\text { sessment (detection bias) } \\
\text { All outcomes }\end{array}$ & Low risk & $\begin{array}{l}\text { Email response: "Subjects, investigators, and research staff were blinded to } \\
\text { the subject treatment assigned during the study." } \\
\text { Comment: Site investigators evaluated efficacy and were blinded to subject } \\
\text { treatment }\end{array}$ \\
\hline
\end{tabular}


Toledo-Bahena 2014 (Continued)

Incomplete outcome data Low risk Comment: The ITT population was clearly specified and used for analyses. At(attrition bias) trition data not included in publication but obtained through email request

All outcomes

Selective reporting (re- High risk porting bias)
Comment: Primary outcomes in protocol used as outcome for publication. Secondary measures differ between the protocol and publication. NCT00679965

\begin{tabular}{|c|c|c|}
\hline Other bias & Low risk & $\begin{array}{l}\text { Comment: Multiple intervention trial, but outcomes for all interventions re- } \\
\text { ported separately }\end{array}$ \\
\hline
\end{tabular}

\section{Tschen 2013}

\section{Methods}

RCT (parallel)

Study design: Double blind

Sample size calculation: No

Diagnosis mycology: $\mathrm{KOH}$ and culture

Big toenail specified: Yes

Statistical comparisons for outcomes: Yes

Duration of follow-up: At 4 weeks

Time points of measurements: Weeks 4, 8, 12, 16, 24, 28, 32, 36 and 40

Location: Mexico - Hosptial Dr. Ángel Leaño / Zapopan, Jalisco; Hospital Ignacio Morones Prieto SLP/ SLP, SLP; Hospital General de México/DF; Unidad de Investigación en Salud/Chihuahua; Hospital Central Militar/DF; Instituto Dermatólogico de Jalisco/Guadalajara; Centro de Dermatología de Monterrey/Monterrey Nuevo León; Hospital Universitario /Monterrey Nuevo León; Hospital regional \# 1 ISSSTE Mérida/Mérida, Yucatán; MIRC/OCA Hospital/Monterrey Nuevo León; Clinical Research Institute/DF

Participants

Total n: 135

Age: Mean, median (range): efinaconazole $10 \%$ with semiocclusion 43.1, 44.5 years (19 - 64), efinaconazole $10 \% 42.7,39.0$ years ( 20 - 63), efinaconazole $5 \% 41.6$, 40.5 years ( $20-63)$, vehicle $44.2,47.0$ years $(25-62)$, total $42.8,42.0$ years $(19-64)$

Sex: Efinaconazole 10\% with semiocclusion: men 16/36 (44.4\%), women 20/36 (55.6\%). Efinaconazole $10 \%$ : men $18 / 39$ (46.2\%), women $21 / 39$ (53.8\%). Efinaconazole 5\%: men 19/38 (50\%), women 19/38 (50\%). Vehicle: men 9/22 (40.9\%), women 13/22 (59.1\%). Total: men 62/135 (45.9\%), women 73/135 $(54.1 \%)$

Inclusion criteria: Age of 18 to 65 years and a clinical diagnosis of DLSO affecting at least 1 great toenail. Mild-to-moderate disease was defined as clinical involvement of $20 \%$ to $50 \%$ of the target toenail, without dermatophytomas or matrix (lunula) involvement. The target toenail had an uninfected length $\geq 3$ $\mathrm{mm}$ (from the proximal nail fold), thickness $\leq 3 \mathrm{~mm}$, and evidence of toenail growth, positive $\mathrm{KOH}$ microscopy and culture of a dermatophyte or mixed dermatophyte/Candida $\leq 42$ days before baseline visit. Women of childbearing potential had to be using effective birth control

Exclusion criteria: A history of immunosuppression or clinical signs or both, indicative of possible immunosuppression, known HIV infection, or uncontrolled diabetes. Additional exclusion criteria included presence of toenail infection other than dermatophytes and Candida, severe moccasin-type tinea pedis at the screening or baseline visits, any disease/condition that might have caused toenail abnormalities or interfered with the evaluation, and previous target toenail surgery. People were not excluded for concomitant drugs that inhibit cytochrome P450 3A4

Disease duration: Unknown 
Tschen 2013 (Continued)

Comparable at baseline: Yes

Causative species: dermatophyte or mixed dermatophyte/Candida

Number of participants lost to follow-up: Lost to follow-up $(n=8,5.9 \%)$, participant request $(n=3$, $2.2 \%)$ and $\operatorname{AEs}(n=3,2.2 \%)$. Reasons for discontinuation-efinaconazole with semi-occlusion: lost to follow-up $n=4$

Trial duration: October 2007 - February 2009

Treatment duration: once daily for 36 weeks
Drug in study arm 1: Efinaconazole $10 \%$ with semi-occlusion, $\mathrm{n}=36$
Drug in study arm 2: Efinaconzole $10 \%, \mathrm{n}=39$
Drug in study arm 3: Efinaconazole $5 \%, \mathrm{n}=38$
Drug in study arm 4: Vehicle, $\mathrm{n}=22$

\begin{tabular}{ll}
\hline Outcomes & Mycological cure ( $\mathrm{KOH}$ and culture) \\
Clinical efficacy (an affected target toenail area of $<20 \%)$ at weeks 24,36 and FU \\
Complete cure ( $0 \%$ clinical involvement of the target nail in addition to a mycological cure) at weeks \\
24,36 and FU \\
Effective treatment (mycological cure and either an affected target toenail area of $0 \%$ or $>3$ mm proxi- \\
mal nail growth from baseline in the unaffected target toenail) at weeks 24,36 and FU \\
Adverse events: reported \\
\hline Notes \\
$\begin{array}{l}4 \text { of } 6 \text { authors are employees of industry, while the remaining } 2 \text { have ties to industry (e.g. investigator). } \\
\text { The study was funded by industry. }\end{array}$
\end{tabular}

\section{Risk of bias}

Bias Authors' judgement Support for judgement

Random sequence genera- Low risk Quote: "Subjects were randomised according to a computer-generated rantion (selection bias) $\quad$ domisation schedule."

Comment: Specific randomisation method that is suitable was specified

\begin{tabular}{ll}
\hline $\begin{array}{l}\text { Allocation concealment Low risk } \\
\text { (selection bias) }\end{array}$ & $\begin{array}{l}\text { Quote: "After the completion of the study and after the database was locked, } \\
\text { the code was provided to data management for identification of the drug as- } \\
\text { signment for each subject." }\end{array}$
\end{tabular}

Comment: Allocation concealment was appropriate

$\begin{array}{ll}\begin{array}{l}\text { Blinding of participants } \\ \text { and personnel (perfor- }\end{array} & \begin{array}{l}\text { Email response: "While the study drugs were provided as identical solutions } \\ \text { packaged in identical bottles, the study personnel who dispensed the medica- } \\ \text { mall outcomes }\end{array} \\ \begin{array}{l}\text { tions would have known which subjects were assigned to the occlusive treat- } \\ \text { ment arm; subjects within this treatment arm similarly would have known } \\ \text { their test article assignment. Therefore, to maintain the integrity of the blind, } \\ \text { the study staff at each site took precautions to ensure that the investigator did } \\ \text { not have access to the study drug, study drug labelling, or any item that identi- } \\ \text { fied individual study drug assignments except in the event of a medical emer- } \\ \text { gency." } \\ \text { Comment: Assessed as low risk. Only } 1 \text { of } 4 \text { treatment arms was known, and } \\ \text { the control group remained unknown to all personnel }\end{array}\end{array}$


Tschen 2013 (Continued)

Blinding of outcome as sessment (detection bias)

All outcomes
Low risk See response above

Email response: "all sponsor and contract research organization personnel who entered, analysed, or otherwise managed subject-specific data were blinded throughout the study."

Comment: Blinding was adequate

Comment: Reasons for participants discontinuing the study were provided for each treatment group. The ITT population was clearly specified (attrition bias)

All outcomes

Selective reporting (re- High risk
porting bias)
porting bias)

Comment: Trial protocol reports one primary and one secondary outcome. Publication does not differentiate primary and secondary outcomes and includes additional outcomes. NCT00777868

\begin{tabular}{ll}
\hline Other bias $\quad$ Low risk & $\begin{array}{l}\text { Comment: Multiple intervention trial, but outcomes for all interventions re- } \\
\text { ported separately }\end{array}$
\end{tabular}

Waibel 2013

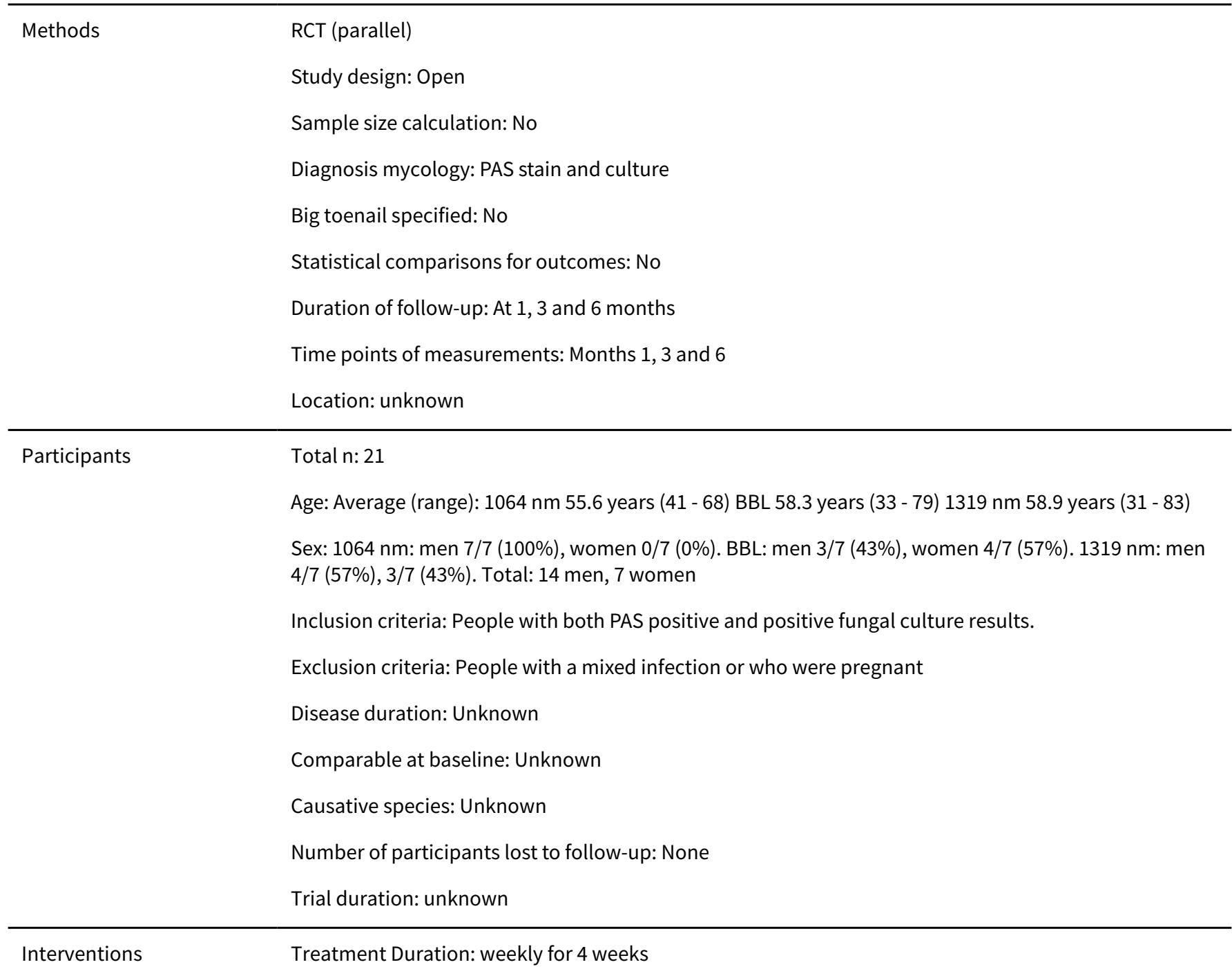


Waibel 2013 (Continued)

Treatment in study arm 1: $1064 \mathrm{~nm}$ laser, $\mathrm{n}=7$

Treatment in study arm 2: $1319 \mathrm{~nm}$ laser, $\mathrm{n}=7$

Treatment in study arm 3: BroadBand Light from a filtered flash lamp, $n=7$

\begin{tabular}{ll}
\hline Outcomes & $\begin{array}{l}\text { Mycological cure (culture) } \\
\text { Clinical endpoint (negative culture) } \\
\text { Adverse events: reported }\end{array}$ \\
\hline Notes & $\begin{array}{l}2 \text { of } 3 \text { authors state no relevant conflicts to disclose. } 1 \text { author has industry ties and is a "co-inventor of } \\
\text { intellectual property...and may gain royalties from future commercialisation of the technology" }\end{array}$ \\
\hline
\end{tabular}

\section{Risk of bias}

\begin{tabular}{lll}
\hline Bias & Authors' judgement & Support for judgement \\
\hline $\begin{array}{l}\text { Random sequence genera- } \\
\text { tion (selection bias) }\end{array}$ & Unclear risk & $\begin{array}{l}\text { Quote: "Patients were randomly assigned to one of three treatment groups" } \\
\text { Comment: Method of randomisation not specified }\end{array}$ \\
\hline $\begin{array}{l}\text { Allocation concealment } \\
\text { (selection bias) }\end{array}$ & Unclear risk & Comment: No information was available \\
\hline $\begin{array}{l}\text { Blinding of participants } \\
\text { and personnel (perfor- } \\
\text { mance bias) } \\
\text { All outcomes }\end{array}$ & High risk & Comment: Open trial \\
\hline
\end{tabular}

\begin{tabular}{lll}
\hline $\begin{array}{l}\text { Blinding of outcome as- } \\
\text { sessment (detection bias) } \\
\text { All outcomes }\end{array}$ & High risk & Comment: Open trial \\
\hline $\begin{array}{l}\text { Incomplete outcome data } \\
\text { (attrition bias) } \\
\text { All outcomes }\end{array}$ & Low risk & Comment: 21 participants entered the study and 21 completed the study \\
\hline $\begin{array}{l}\text { Selective reporting (re- } \\
\text { porting bias) }\end{array}$ & Unclear risk & $\begin{array}{l}\text { Comment: Review authors were unable to find a clinical trial register entry for } \\
\text { this study }\end{array}$ \\
\hline $\begin{array}{l}\text { Other bias } \\
\text { Comment: Multiple intervention trial, but outcomes for all interventions re- }\end{array}$ & ported separately \\
\hline
\end{tabular}

Watanabe 2017

$\begin{array}{ll}\text { Methods } & \text { RCT (parallel) } \\ \text { Study design: Double blind } \\ \text { Sample size calculation: Yes } \\ \text { Diagnosis mycology: } \mathrm{KOH} \text { and LAMP } \\ \text { Big toenail specified: Yes } \\ \text { Statistical comparisons for outcomes: Yes } \\ \text { Duration of follow-up: At } 52 \text { weeks }\end{array}$


Watanabe 2017 (Continued)

Time points of measurements: Weeks 4,48 and 52

Location: Japan (cities/institutions unknown)

Participants Total n: 293

Age: Mean, median (range): luliconazole 56.9, 59.0 years ( 24 - 79), vehicle 58.9, 62.0 years (21 - 78), total $57.6,60.0$ years $(21-79)$

Sex: Luliconazole: men 129/194 (66.5\%), women 65/194 (33.5\%). Vehicle: men 76/99 (76.8\%), women 23/99 (23.2\%). Total: men 205/293 (70.0\%), women 88/293 (30.0\%)

Inclusion criteria: The participants had to: (i) be at least 20 years of age but < 80 years; (ii) have distal lateral subungual onychomycosis involving either the left or the right great toenail; (iii) have positive $\mathrm{KOH}$ test results under direct microscopy, and positive detection of T. rubrum or

T. mentagrophytes by the LAMP method; (iv) have onychomycosis-related clinical involvement affecting $20 \%-50 \%$ of the nail, with a nail thickness of $<3 \mathrm{~mm}$; and $(\mathrm{v})$ provide written informed consent

Exclusion criteria: (i) had a history of hypersensitivity to an antifungal agent; (ii) had a history of outpatient treatment for contact dermatitis caused by a topical agent; (iii) had a history of narcotic/drug abuse or alcohol addiction or both; (iv) had severe heart, liver, kidney, lung or blood disease(s) or diabetes; (v) showed disease involvement near the proximal nail fold; (vi) had longitudinal streaks or a spike; (vii) had abnormal conditions in the target toenail that may affect evaluation of drug efficacy; (viii) were unlikely to obtain natural growth of the nail; (ix) had used an oral/injectable antifungal agent within 36 weeks before the start of this study (at baseline); ( $\mathrm{x}$ ) had applied a topical antifungal agent to the nail within 4 weeks before the start of this study (at baseline); (xi) were using or scheduled to use an adrenocortical hormone preparation, immunosuppressant, anti-cancer drug, skin emollient or anti-keratosis agent; (xii) were pregnant or suspected of being pregnant, were nursing or wished to become pregnant during the study period; or (xiii) were judged by the physicians in charge of the study to be ineligible to participate

Disease duration: Unknown

Comparable at baseline: Analysis of the participant disposition showed no significant differences between the 2 groups in any of the relevant demographic factors

Causative species: Trichophyton rubrum: luliconazole 148/194 (76.3\%); vehicle 76/99 (76.8\%); total 224/293 (76.5\%). T. mentagrophytes: luliconazole 46/194 (23.7\%); vehicle 23/99 (23.2\%); total 69/293 (23.5\%)

Number of people lost to follow-up: Poor response to study drug or worsening of symptoms: luliconazole $n=4$, vehicle $n=2$. Development of adverse events: luliconazole $n=12$, vehicle $n=2$. Worsening of complications: luliconazole $n=1$. Observation/laboratory tests could not be performed: luliconazole $n=3$, vehicle $n=2$. Non-compliance (use of prohibited concomitant drug): luliconazole $n=1.1$ participant in the luliconazole group displayed 2 reasons simultaneously: poor response to study drug or worsening of symptoms and development of adverse events.

Trial duration: April 2012 - January 2014

\begin{tabular}{ll} 
Interventions & Treatment duration: Once daily for 48 weeks \\
& Drug in study arm 1: Luliconazole $5 \%$ nail solution, $\mathrm{n}=194$ \\
& Drug in study arm 2: Vehicle, $\mathrm{n}=99$ \\
\hline Outcomes & Mycological cure $(\mathrm{KOH})$ at weeks 4,48 and 52 \\
& Complete cure $(0 \%$ clinical involvement of the nail with negative results on direct microscopy) at week \\
48 & Adverse events: reported
\end{tabular}


Watanabe 2017 (Continued)

Notes 2 of 3 authors are employees of industry. The study was funded by industry

\section{Risk of bias}

Bias Authors' judgement Support for judgement

Random sequence genera- Low risk
tion (selection bias)

Quote: "randomised...using the permuted block method with six subjects per block"

Comment: Specific randomisation method that is suitable was specified

\begin{tabular}{|c|c|c|}
\hline $\begin{array}{l}\text { Allocation concealment } \\
\text { (selection bias) }\end{array}$ & Unclear risk & $\begin{array}{l}\text { Quote: "allocation of the study drug was entrusted to a third party not in- } \\
\text { volved in evaluating the study results" }\end{array}$ \\
\hline & & Comment: Unclear if allocation was withheld from study personnel \\
\hline
\end{tabular}

Blinding of participants Unclear risk Comment: Blinded, but unclear if study drugs looked identical

and personnel (perfor-

mance bias)

All outcomes

\begin{tabular}{lll}
\hline $\begin{array}{l}\text { Blinding of outcome as- } \\
\text { sessment (detection bias) }\end{array}$ & Unclear risk & $\begin{array}{l}\text { Comment: Blinded, but no specific mention of outcome assessor blinding } \\
\text { method }\end{array}$ \\
All outcomes &
\end{tabular}

\begin{tabular}{lll}
\hline $\begin{array}{l}\text { Incomplete outcome data } \\
\text { (attrition bias) } \\
\text { All outcomes }\end{array}$ & Low risk & $\begin{array}{l}\text { Comment: Reasons for participants discontinuing the study were provided for } \\
\text { each treatment group. The ITT population was clearly specified }\end{array}$ \\
\hline $\begin{array}{l}\text { Selective reporting (re- } \\
\text { porting bias) }\end{array}$ & High risk & $\begin{array}{l}\text { Comment: Trial protocol reports one outcome, complete cure, without a de- } \\
\text { finition. The publication defines complete cure and includes additional sec- } \\
\text { ondary endpoints. NCT01431820 }\end{array}$ \\
\hline Other bias & Low risk & $\begin{array}{l}\text { Comment: Not a multiple intervention trial, with all outcomes reported for in- } \\
\text { terventions }\end{array}$ \\
\hline
\end{tabular}

AEs: adverse events; DLSO: distal lateral subungual onychomycosis; FU: follow-up; ISGA: Investigator's Static Global Assessment; ITT: intention to treat; $\mathrm{KOH}$ : potassium hydroxide; LAMP: loop-mediated isothermal amplification; PAS: periodic acid-Schiff; PCR: polymerase chain reaction; RCT: randomised controlled trial; SCIO: scoring clinical index for onychomycosis; TEAEs: treatment-emergent adverse events; TGT: target great toenail.

Characteristics of excluded studies [ordered by study ID]

\begin{tabular}{ll}
\hline Study & Reason for exclusion \\
\hline Abd El-Aal 2019 & Paediatric and adult patient population \\
\hline Anonymous 2000 & Not a RCT \\
\hline Anonymous 2015 & Not a RCT \\
\hline Bassiri-Jahromi 2012 & Not toenails alone \\
\hline Bergstrom 2009 & Not a RCT \\
\hline Botter 1971 & Not a RCT \\
\hline \hline
\end{tabular}




\begin{tabular}{|c|c|}
\hline Study & Reason for exclusion \\
\hline Canavan 2018 & Not a RCT \\
\hline Chan 2005 & Duration less than 12 weeks \\
\hline Dina 2017 & Not a RCT \\
\hline El-Tatawy 2015 & Not toenails alone \\
\hline Emtestam 2012 & Not toenails alone \\
\hline Fredriksson 1974 & Not a RCT \\
\hline Furtado 2005 & Not a RCT \\
\hline Gilaberte 2017 & Not toenails alone \\
\hline Gupta 2005 & Ineligible treatment \\
\hline Halmy 2005 & Not toenails alone \\
\hline Harris 2010 & Within-participant design \\
\hline Hay 1988 & Not a RCT \\
\hline Jimenez-Faraj 2018 & Duration less than 12 weeks \\
\hline Kamalam 1980 & Not onychomycosis \\
\hline Kim 2016a & Nail as unit of analysis \\
\hline Kim 2016b & Not toenails alone \\
\hline Krasaeath 2016 & Not a RCT \\
\hline Landsman 2010 & Nail as unit of analysis \\
\hline Landsman 2012 & Nail as unit of analysis \\
\hline Lauharanta 1992 & Not toenails alone \\
\hline Li 2014 & Not toenails alone \\
\hline Lu 2016 & Nail as unit of analysis \\
\hline Mahgoub 1975 & Not toenails alone \\
\hline Malay 2009 & Nail as unit of analysis \\
\hline Many 1964 & Not toenails alone \\
\hline Mensing 1992 & Duration less than 12 weeks \\
\hline Menéndez 2011 & Not toenails alone \\
\hline Meyerson 1992 & Not a RCT \\
\hline
\end{tabular}




\begin{tabular}{|c|c|}
\hline Study & Reason for exclusion \\
\hline Morgado 2017 & Not a RCT \\
\hline NCT03094468 & Paediatric and adult patient population \\
\hline Oberste-Lehn 1974 & Ineligible treatment \\
\hline Ortiz 2014 & Within-participant design \\
\hline Rand 1996 & Not a RCT \\
\hline Reinel 1992 & Nail as unit of analysis \\
\hline Schaller 2017 & Within-participant design \\
\hline Schlockermann 1957 & Ineligible treatment \\
\hline Shemer 2016 & Not a RCT \\
\hline Sigurgeirsson 2010 & Prophylaxis \\
\hline Sotiriou 2010 & Not a RCT \\
\hline Souza 2014 & Not a RCT \\
\hline Tardivo 2015 & Not a RCT \\
\hline Tietz 2013 & Not toenails alone \\
\hline Zaug 1995 & Not a RCT \\
\hline Zhang 2012a & Not toenails alone \\
\hline Zhou 2016 & Not toenails alone \\
\hline
\end{tabular}

$\mathrm{RCT}$ : randomised controlled trial.

Characteristics of studies awaiting assessment [ordered by study ID]

Anonymous 2002

\begin{tabular}{ll}
\hline Methods & - \\
\hline Participants & - \\
\hline Interventions & - \\
\hline Outcomes & Article not in English and unavailable
\end{tabular}

Methods RCT: unknown


Bagatell 1977 (Continued)

Study design: Double blind

Diagnosis mycology: $\mathrm{KOH}$ and culture

Big toenail specified: Unknown if finger-, toenails, or both

Duration of follow-up: 12 weeks

Time points of measurements: 12 and 24 weeks

Location: unknown

\begin{tabular}{ll}
\hline Participants & Total n: Unknown \\
Age: Unknown \\
Sex: Unknown \\
Inclusion/exclusion criteria: Unknown \\
Number of participants lost to follow-up: 1 \\
Trial duration: unknown \\
Treatment duration: 12 weeks for double-blind; single-blind for weeks 12 - 24 where placebo re- \\
ceived active treatment \\
Drug in study arm 1: Fluorouracil 1\% in propylene glycol solution, $\mathrm{n}=$ unknown \\
Drug in study arm 2: Placebo solution (propylene glycol), $\mathrm{n}=$ unknown \\
\hline Clinical response and laboratory results \\
Adverse events: not reported \\
\hline Letter to the editor. Cannot assess eligibility as letter does not state whether study investigates fin- \\
gernail, toenails, or both
\end{tabular}

\section{Barquero 2007}

\section{Methods}

\section{RCT: unknown}

Study design: Double blind

Diagnosis mycology: culture

Big toenail specified: No

Duration of follow-up: monthly

Time points of measurements: monthly

Location: Nicaragua (city/institution unknown)

Sex: Unknown

Inclusion/exclusion criteria: Unknown 
Trial duration: unknown

\begin{tabular}{ll}
\hline Interventions & Treatment duration: unknown \\
& Drug in study arm 1: terbinafine $1 \%$ gel, $\mathrm{n}=16$ \\
& Drug in study arm 2: placebo gel, $\mathrm{n}=8$ \\
\hline Outcomes & $\begin{array}{l}\text { Success or cure: continuous increase of normal nail bed until a normal nail replaced the infected } \\
\text { nail }\end{array}$ \\
& $\begin{array}{l}\text { Failure: inability, after } 2 \text { months, to increase the length of normal nail bed as compared to initial } \\
\text { visit }\end{array}$ \\
& Adverse events: not reported \\
\hline Cotes & $\begin{array}{l}\text { Conference abstract. Cannot assess eligibility as age of participants and whether randomisation } \\
\text { occurred is noted }\end{array}$ \\
\hline
\end{tabular}

\section{Butareva 1986}

\begin{tabular}{ll}
\hline Methods & - \\
\hline Participants & - \\
\hline Interventions & - \\
\hline Outcomes & Article not in English and unavailable
\end{tabular}

Emokpare 1977

Methods

\section{Participants}

Interventions

\section{Outcomes}

$\begin{array}{lll}\text { Notes Article not in English } & \text { Arta }\end{array}$

\section{EUCTR2008-002427-90

Methods RCT (parallel) \\ Study design: Double blind \\ Sample size calculation: Unknown}


Diagnosis mycology: Unknown

Big toenail specified: No

Statistical comparison for outcome: Unknown

Duration of follow-up: Unknown

Time points of measurement: 6 or 9 months

Location: France (city/institution unknown)

\section{Participants}

\section{Total n: 300}

Age: 18 - 64 years old

Sex: Men and women (ratio unknown)

Inclusion criteria: Men and women, 18 - 64 years of age, affiliated to a French social security scheme or benefiting from such a scheme, presenting with nail onychomycosis without matrix involvement of a finger or toe that has not yet been treated with an antimycotic

Exclusion criteria: Disease or condition requiring immunosuppressive therapy, known allergies or contraindications to investigative products, 2+ fingers or toes affected, oral mycotic treatment, inability to consent, ineffective contraception, assessed as unlikely to comply with study requirements

Disease duration: Unknown

Comparable at baseline: Unknown

Causative species: Unknown

Number of people lost to follow-up: Unknown

Trial duration: unknown
Treatment duration: Flngers for 6 months and toes for 9 months

Drug in study arm 1: amorolfine, generic 5\%, film-forming solution, $n=$ unknown

Drug in study arm 2: Loceryl ${ }^{\circledR}$, amorolfine 5\%, n = unknown.

Outcomes Clinical and mycological efficacy following 6 months (fingers) or 9 months (toes)

Adverse events: not reported

Notes

Method of diagnosing onychomycosis not specified

\section{George 1991}

\begin{tabular}{ll}
\hline Methods & - \\
\hline Participants & - \\
\hline Interventions & - \\
\hline Outcomes & Abstract and full text not available \\
\hline Notes & \\
\hline
\end{tabular}


Grant 1974

\section{Methods}

Participants

Interventions

\section{Outcomes}

\section{Notes}

\begin{tabular}{|c|c|}
\hline \multirow[t]{9}{*}{ Methods } & $\mathrm{RCT}$ (parallel) \\
\hline & Study design: Double blind \\
\hline & Sample size calculation: Unknown \\
\hline & Diagnosis mycology: Direct microscopy \\
\hline & Big toenail specified: Unknown if finger-, toenails, or both \\
\hline & Statistical comparison for outcome: Unknown \\
\hline & Duration of follow-up: Unknown \\
\hline & Time points of measurements: Unknown \\
\hline & Location: Japan (city/institution unknown) \\
\hline \multirow[t]{10}{*}{ Participants } & Total n: Unknown \\
\hline & Age: 20 - 79 years old \\
\hline & Sex: Unknown. \\
\hline & $\begin{array}{l}\text { Inclusion criteria: People with turbidity on the nails. People with dermatophytes on the nails by di- } \\
\text { rect microscopy. People with dermatophytes of the nails aged } 20 \text { - } 79 \text { years old, both genders eligi- } \\
\text { ble }\end{array}$ \\
\hline & $\begin{array}{l}\text { Exclusion criteria: People who used antifungal agents of oral preparations and injections within } \\
36 \text { weeks before consent acquisition, were used up to the test site and the surrounding first joint } \\
\text { within } 4 \text { weeks before consent acquisition. People who used external antifungal agent containing } \\
\text { terbinafine hydrochloride within } 12 \text { weeks before consent acquisition }\end{array}$ \\
\hline & Disease duration: Unknown \\
\hline & Comparable at baseline: Unknown \\
\hline & Causative species: Dermatophyte \\
\hline & Number of participants lost to follow-up: Unknown \\
\hline & Trial duration: unknown \\
\hline
\end{tabular}

Interventions

Treatment duration: Once a day for an unknown amount of time

Drug in study arm 1: HTU-520 (patch containing terbinafine hydrochloride), $\mathrm{n}=$ unknown 
JPRN-JapicCTI-101143 (Continued)

Drug in study arm 2: HTU-520 placebo applied once a day, $\mathrm{n}=$ unknown

\begin{tabular}{ll} 
Outcomes & Efficacy \\
& Adverse events: not reported \\
\hline Notes & Cannot assess eligibility as does not state whether study investigates fingernail, toenails, or both \\
\hline
\end{tabular}

\section{Klima 1976}

Methods

\section{Participants}

Interventions

\section{Outcomes}

Notes Article not in English and unavailable

Kull 1972

Methods

Participants

Interventions

\section{Outcomes}

$$
\text { Notes }
$$

Article not in English and unavailable

\section{Mathur 1973}

Methods

\section{Participants}

Interventions

\section{Outcomes}

Notes

Abstract and full text not available

NCT00253305

$\begin{array}{ll}\text { Methods } & \text { RCT (parallel) } \\ \text { Study design: Double blind }\end{array}$


NCT00253305 (Continued)

\section{Sample size calculation: Unknown}

Diagnosis mycology: $\mathrm{KOH}$ and culture

Big toenail specified: Yes

Statistical comparisons for outcomes: Unknown

Duration of follow-up: Unknown

Time points of measurements: Unknown

Location: United States (University of Alabama at Birmingham, Birmingham, Alabama; Genova Clinical Research, Inc., Tucson, Arizona; Greater Miami Skin and Laser Center, Miami Beach, Florida; Washington University, St. Louis, Missouri; Northwest Cutaneous Research, Portland, Oregon)

\section{Participants}

\section{Total n: 75}

Age: $18-65$ years of age

Sex: Men and women (ratio unknown)

Inclusion criteria: Clinical diagnosis of distal subungual onychomycosis of one great toenail with $20 \%$ - 65\% nail involvement inclusive, positive $\mathrm{KOH}$ microscopy and culture for a dermatophyte, 2 $\mathrm{mm}$ clear nail proximally, women post-menopausal or agree to appropriate contraceptives

Exclusion criteria: Infecting organisms other than dermatophytes, proximal subungual onychomycosis, spikes of disease extending to nail matrix, $5+$ infected nails, confounding problems/abnormalities of target nail, screening lab values more than $20 \%$ of normal, hypersensitivity to test materials, systemic medications, poor history of compliance

Disease duration: Unknown

Comparable at baseline: Unknown

Causative species: dermatophytes

Number of people lost to follow-up: Unknown

Trial duration: start September 2005, completion date unknown

Drug in study arm 1: Organogel of naftifine, $2 \%$

Drug in study arm 2: Organogel of terbinafine, $2 \%$

Drug in study arm 3: Organogel of naftifine, $6 \%$

Drug in study arm 4: Organogel of terbinafine, $6 \%$

$\mathrm{KOH}$ and culture

Time to achieve $90 \%$ and $100 \%$ clearance of fungus from the nail

Treatment success

Mycological success

Adverse events: not reported 


\section{Methods \\ Methods}

(2)

\section{RCT (parallel)}

Study design: Double blind

Sample size calculation: Unknown

Diagnosis mycology: Culture and $\mathrm{KOH}$

Big toenail specified: Yes

Statistical comparison for outcome: Unknown

Duration of follow-up: Unknown

Time point of measurement: At week 60

Location: multiple locations in Belgium, Bulgaria, Czechia, Germany, Greece, Hungary, Iceland, Latvia, Lithuania, Poland, Russian Federation, Slovakia, and Sweden (cities/institutions unknown)
Total n: 980

Age: 12 years and older

\section{Sex: Unknown}

Inclusion criteria: Written informed consent, people aged 12 years and older of any race, male or female, people with onychomycosis involving $\geq 20 \%$ to $\leq 50 \%$ of target big toenail, people with a positive $\mathrm{KOH}$ examination and culture positive for dermatophyte

Exclusion criteria: Presence of 'yellow spikes' on the target nail, presence of dermatophytoma on the target nail, presence of nail thickness exceeding $2 \mathrm{~mm}$, people with proximal subungual involvement, people with severe plantar or moccasin tinea pedis, people with nail abnormalities due to other conditions, people with life expectancy $<2$ years, chemotherapy, immunosuppressive therapy in the 12 weeks prior to screening visit (V1), systemic corticosteroids, antimetabolites and immune-stimulants therapy in the 4 weeks prior to Screening visit (V1), HIV infection or any other immunodeficiency, alcohol or substance abuse, people with history of allergic reactions to terbinafine or its excipients, women who are pregnant, nursing an infant, or planning a pregnancy during the study period

Disease duration: Unknown

Comparable at baseline: Unknown

Causative species: Dermatophyte

Number of people lost to follow-up: Unknown

Trial duration: August 20, 2015 - September 17, 2018

Interventions

Treatment duration: For 60 weeks

Drug in study arm 1: Drug: P-3058 10\%, n = unknown

Drug in study arm 2: Vehicle of P-3058 10\%, n = unknown

Drug in study arm 3: Amorolfine 5\% (active comparator), $n=$ unknown

Outcomes

Complete cure at week 60

Adverse events: not reported 
NCT02549001 (Continued)

Notes

Industry provided financial support. Last participant completed 17 September 2018. Unknown if under 18 years of age and over 18 years of age will be reported separately

Park 2017

Methods

RCT (parallel)

Study design: Evaluator-blind

Sample size calculation: Yes

Diagnosis mycology: $\mathrm{KOH}$ and culture

Big toenail specified: No

Statistical comparisons for outcomes: Yes

Duration of follow-up: None

Time points of measurements: weeks 4, 8, 12, 16

Location: Department of Dermatology, Chung-Ang University Hospital, Seoul, Korea

\begin{tabular}{|c|c|}
\hline \multirow[t]{10}{*}{ Participants } & Total n: 128 \\
\hline & Age: $>19$ years of age \\
\hline & Sex: Men and women (ratio unknown) \\
\hline & $\begin{array}{l}\text { Inclusion criteria: Men and women over } 19 \text { years of age, with onychomycosis confirmed with posi- } \\
\text { tive } \mathrm{KOH} \text { smear }\end{array}$ \\
\hline & $\begin{array}{l}\text { Exclusion criteria: History of photosensitivity, allergic reaction to amorolfine, other prediagnosed } \\
\text { nail disorders such as nail psoriasis, breastfeeding/pregnancy, or undergoing topical/systemic anti- } \\
\text { fungal agent or laser treatments within } 12 \text { weeks before the study }\end{array}$ \\
\hline & Disease duration: Unknown \\
\hline & Comparable at baseline: Yes \\
\hline & Causative species: dermatophytes \\
\hline & Number of people lost to follow-up: None \\
\hline & Participant enrollment: May 2014 - March 2015 \\
\hline \multirow[t]{2}{*}{ Interventions } & $\begin{array}{l}\text { Treatment in study arm 1: 1064-nm Nd:YAG laser treatment, once every } 4 \text { weeks for } 4 \text { treatments, } \\
\text { and concurrent amorolfine } 5 \% \text { nail lacquer once a week for } 16 \text { weeks, } n=64\end{array}$ \\
\hline & Drug in study arm 2: 5\% amorolfine nail lacquer once a week for 16 weeks, $n=64$ \\
\hline \multirow[t]{5}{*}{ Outcomes } & Mycological cure: negative $\mathrm{KOH}$ and culture at 16 weeks \\
\hline & $\begin{array}{l}\text { Cumulative cure: clinically normal nail or negative mycology with }<10 \% \text { nail plate involvement at } \\
16 \text { weeks }\end{array}$ \\
\hline & Change in lesion-free area (\%) from baseline at 16 weeks \\
\hline & Participant satisfaction on a 5-point Likert scale at 16 weeks \\
\hline & Adverse events: reported \\
\hline
\end{tabular}


Park 2017 (Continued)
Notes
Toenails alone unknown

Ramm 1994

Methods

Participants

Interventions

\section{Outcomes}

Notes Abstract and full text not available

Rüping 1993a

Methods

Participants

Interventions

\section{Outcomes}

Notes

Article not in English and unavailable

Rüping 1993b

\begin{tabular}{l} 
Methods \\
\hline Participants
\end{tabular}

Interventions

\section{Outcomes}

Notes

Abstract and full text not available

\section{Schubert 1973}

\section{Methods}

\section{Participants}

\section{Interventions}

\section{Outcomes}


Schubert 1973 (Continued)

Notes

Article not in English and unavailable

Weuta 1972

Methods

\section{Participants}

Interventions

\section{Outcomes}

Notes Article not in English and unavailable

\section{Xu 2017}

\section{Methods}

\section{Participants}

\begin{tabular}{ll}
\hline Interventions & - \\
\hline Outcomes & - \\
\hline Notes & $\begin{array}{l}\text { Article not in English and unavailable. To be assessed for inclusion/exclusion in an update to the re- } \\
\text { view. Added to review as part of updated literature search during review process }\end{array}$ \\
\hline
\end{tabular}

Yao 2016

$\begin{array}{ll}\text { Methods } & \text { RCT: Yes } \\ \text { Study design: unknown } \\ \text { Diagnosis mycology: Unknown } \\ \text { Big toenail specified: No } \\ \text { Duration of follow-up: } 9 \text { months } \\ \text { Time points of measurements: } 13 \text { months } \\ \text { Location: unknown } \\ \text { Total n: } 60 \\ \text { Age: exact ages unknown, described as "middle and aged" } \\ \text { Sex: Unknown } \\ \text { Inclusion/exclusion criteria: Unknown } \\ \text { Number of participants lost to follow-up: Unknown }\end{array}$


Trial duration: unknown

Interventions Treatment duration: 4 months

Treatment in study arm 1: Fufang Tujin Piding topical twice daily with 1064-nm Nd:YAG laser once weekly, $\mathrm{n}=30$

Treatment in study arm 2: 1064-nm Nd:YAG laser once weekly, $\mathrm{n}=30$

\begin{tabular}{ll}
\hline Outcomes & Clinical efficacy 9 months following treatment \\
& Mycological efficacy 9 months following treatment \\
& Adverse events: not reported \\
\hline Notes & $\begin{array}{l}\text { Conference abstract. Cannot assess eligibility as does not state whether study investigates finger- } \\
\text { nail, toenails, or both and does not state how onychomycosis was diagnosed }\end{array}$
\end{tabular}

$\mathrm{KOH}$ : potassium hydroxide.

Characteristics of ongoing studies [ordered by study ID]

ACTRN12618000131257

Trial name or title
Phase 1, first in human $(\mathrm{FIH})$, randomised, double-blind, placebo-controlled multiple ascending dose study in healthy volunteers with mild-to-moderate onychomycosis

\begin{tabular}{|c|c|}
\hline \multirow[t]{8}{*}{ Methods } & RCT (parallel) \\
\hline & Study design: Double blind \\
\hline & Sample size calculation: Unknown \\
\hline & Diagnosis mycology: $\mathrm{KOH}$ and/or culture \\
\hline & Big toenail specified: Yes \\
\hline & Statistical comparisons for outcomes: Unknown \\
\hline & Duration of follow-up: 52 weeks \\
\hline & Time points of measurement: $3,6,12$ months \\
\hline
\end{tabular}

Participants

Total n: 48

Age: 18 - 65 years old

Sex: Men and women (ratio unknown)

Inclusion criteria: Men and women, 18 to 65 years of age inclusive, diagnosed with onychomycosis of the great toenail, involving at least $20 \%$, but not more than $70 \%$ of nail involvement, confirmed by mycological staining or culture or both, thickness of distal nail plate $<3 \mathrm{~mm}$, medically healthy, negative urine drug screen/alcohol breath test at screening and day 1, BMI of 17.5 - 35 inclusive, use of double barrier contraception during the study and for 1 month (women) and 3 months (men) after the study or surgical sterilisation at least 6 months prior to screening or abstinence, men must not donate sperm for at least 3 months after the last drug dose, partners of all participants must use contraception, not be pregnant, or be post-menopausal

Exclusion criteria: History of allergy to any of the excipients in HXP124, positive test for HIV, hepatitis $B$ surface antigen, hepatitis $C$ antibodies, underlying conditions that prevent compliance (investigator's discretion), pregnancy or lactation, unwillingness to refrain from nail cosmetics during study, administration of another trial's product within 30 days of study, diabetes mellitus requir- 
ing treatment other than diet/exercise, not adhering to washout periods or concurrent use during study of topical antifungals applied to feet (washout: 4 weeks) apart from that for tinea pedis or anti-inflammatories, corticosteroids, and topical immunomodulators (washouts: 2 weeks), not adhering to washout periods or concurrent use during study of systemic corticosteroids (washout: 2 weeks) or systemic antifungals with known activity against dermatophytes (washout: 12 weeks), use of systemic immunomodulators in the past 4 weeks, nail or anatomic abnormalities of the toe

Disease duration: Unknown

Comparable at baseline: Unknown

Causative species: Unknown

Number of people lost to follow-up: Unknown

Interventions Treatment duration: once daily for 42 days

Cohort 1: HXP124, $5 \mathrm{mg} / \mathrm{mL}, \mathrm{n}=4$; Placebo, $\mathrm{n}=2$

Cohort 2: HXP124, $10 \mathrm{mg} / \mathrm{mL}, \mathrm{n}=4$; Placebo, $\mathrm{n}=2$

Cohort 3: HXP124, $20 \mathrm{mg} / \mathrm{mL}, \mathrm{n}=4$; Placebo, $\mathrm{n}=2$

Cohort 4: HXP124, $n=24$; Placebo, $n=6$ (dose for cohort 4 depends on results in cohorts 1 - 3 )

Outcomes $\quad$ Mycological cure: negative $\mathrm{KOH}$ and culture at days $14,42,63$, and 84 following the first dose

Clinical appearance (photographs), including length and surface area of clear nail growth at days 7 , $14,42,63,84$ following the first dose, plus day 365 for cohort 4

Safety and tolerability: vital signs and adverse events prior to first dose, and $0.5,1,3,5,7,10,15$, and 24 hours, and day $3,7,14,42,63$, and 84 following dosing, plus day 365 for cohort 4; physical exam and ECG prior to first dose, and 24 hours (exam only), day 3, 7, 14, 42, 63, and 84 after dosing; pain, erythema, and irritation assessment prior to first dose, and 60 mins, 4 hours (cohort 4 ), 7 and 24 hours (cohorts 1 - 3), and on day 3, 7, 14, 42, 63, and 84 after first dose; blood collected prior to first dose and on day $7,14,42,63$, and 84 after first dose

Pharmacokinetic parameters: Blood samples collected prior to first dose, and $0.5,1,3,5,7,10,15$, and 24 hours (cohorts $1-3$ ) and day $3,7,14$, and 42 following the first dose

Adverse events: not reported

Starting date Date of first participant enrolment 22 January 2018

Contact information Liisa Bevan, Linear Clinical Research

Australia

lbevan@linear.org.au

Location QEll Medical Centre, Nedlands, WA, Australia

Notes

Recruiting, Estimated study completion 19 May 2019, Last updated on 14 February 2018 
ChicTR-INR-17013504 (Continued)

RCT (parallel)
Study design: Single blind
Sample size calculation: Unknown
Diagnosis mycology: microscopy
Big toenail specified: No
Statistical comparisons for outcomes: Unknown
Duration of follow-up: 12 weeks
Time points of measurement: 12 weeks

Participants

Total n: 266

Age: 18 - 65 years old

Sex: Men and women (ratio unknown)

Inclusion criteria: Men and women, 18 to 65 years of age, diagnosed with onychomycosis, confirmed by clinical symptoms and positive fungal microscopy, an onychomycosis severity index score of $>1$, and effective contraception for women of childbearing age during the trial period

Exclusion criteria: Known history of allergy to romero liniment, severe immunosuppression or use of systemic glucocorticoids and immunosuppressive agents within 3 months, severe heart, liver, or kidney disease, severe neurological or mental illness, poorly-controlled diabetes, antifungal use within last 3 months, women planning to conceive or unwilling to use contraception, history or drug abuse, clinical trial participation within 3 months Disease duration: Unknown

Comparable at baseline: Unknown

Causative species: Unknown

Number of people lost to follow-up: Unknown

Intervention in study arm 1: 2940-nm Er:YAG fractional laser combined with amorolfine hydrochloride, $n=133$

Intervention in study arm 2: amorolfine hydrochloride, $\mathrm{n}=133$

\begin{tabular}{ll}
\hline Outcomes & Mycological cure at 12 weeks \\
& Adverse events: not reported \\
\hline Starting date & 01 January 2018 \\
\hline Contact information & Sun Yat-sen Memorial Hospital of Sun Yat-sen University \\
\hline Location & Sun Yat-sen Memorial Hospital of Sun Yat-sen University, Guangzhou, Guangdong, China \\
\hline Notes & Ongoing. Estimated study completion December 2019, Last update 23 November 2017
\end{tabular}

\section{DRKS00007709}


DRKS00007709 (Continued)

RCT (parallel)
Study design: Double blind
Sample size calculation: Unknown
Diagnosis mycology: Culture and PCR
Big toenail specified: No
Statistical comparisons for outcome: Unknown
Duration of follow-up: At 3 months
Time points of measurements: Month 3

Participants

Total n: 15

Age: 18 - 90 years old

Sex: Unknown

Inclusion criteria: Both genders, aged 18 - 90 years old with onychomycosis of toenail, positive fungal culture, positive PCR, legal age and written agreement

Exclusion criteria: Oral or topical anti-fungal agents in the preceding 6 months, non-compliance, pregnancy, women of childbearing age or lactation and use of a legal carer

Disease duration: Unknown

Comparable at baseline: Unknown

Causative species: Unknown

Number of people lost to follow-up: Unknown

Interventions

Treatment duration: Laser treated 4 times for 4 - 6 weeks and applied once daily creme for 12 months

Treatment in study arm 1: Short-pulsed Nd:YAG Laser-treatment and amorolfin creme, study control, $\mathrm{n}=$ unknown

Drug in study arm 2: Amorolfin creme, study duration and control, $\mathrm{n}=$ unknown

\begin{tabular}{ll}
\hline Outcomes & Clinical cure at month 3 \\
& Mycological cure (fungal culture and PCR) at month 3 \\
& Adverse events: not reported \\
\hline Starting date & Date of registration in DRKS: 20 January 2015 \\
\hline Contact information & $\begin{array}{l}\text { Address: Laserklinik Karlsruhe Kaiserstrasse } 10476133 \text { Karlsruhe Germany } \\
\text { Primary sponsor URL: www.raulin-und-kollegen.de }\end{array}$ \\
\hline Location & Laserklinik Karlsruhe Kaiserstrasse 104 76133 Karlsruhe Germany \\
\hline Notes & Investigator-initiated trial, no commercial funding \\
\hline
\end{tabular}


Trial name or title A multi-centre, randomised, two-armed, parallel group and evaluator-blinded study of efficacy and safety of topical MOB015B in the treatment of mild-to-moderate distal subungual onychomycosis (DSO)

Rethods
Study design: Single blind
Sample size calculation: Unknown
Diagnosis mycology: Culture
Big toenail specified: Yes
Statistical comparisons for outcome: Unknown
Duration of follow-up: Unknown
Time points of measurements: Visit $1-7$

Participants Total n: 406

Age: $n=36618-64$ years old; $n=40 ;>65$ years old

Sex: Unknown

Inclusion criteria: Men or women 18 - 75 years of age (inclusive) at the time of Informed consent, DSO of at least one of the great toenail(s) affecting $20 \%$ to $60 \%$ of the target nail, positive culture for dermatophytes, signed written informed consent (ICF) prior to any study-related activity

Exclusion criteria: PSO, DSO of both great toenails where involvement has extended into the proximal portion of the target nail (unaffected proximal nail is $<3 \mathrm{~mm}$ ), target toenail thickness more than $3 \mathrm{~mm}$, 'spike' of onychomycosis extending to eponychium of the target toenail, presence of dermatophytoma (defined as thick masses of fungal hyphae and necrotic keratin between the nail plate and nail bed) on the target nail, other conditions than DSO known to cause abnormal nail appearance, presence of toenail infection other than dermatophytes, previous target toenail surgery with any residual disfigurement, topical treatment of the nails with other antifungal medication or medical device within 6 weeks before Screening/Visit 1, systemic use of antifungal treatment within 6 months before Screening/Visit 1, severe moccasin tinea pedis, signs of severe peripheral circulatory insufficiency, uncontrolled diabetes mellitus (blood glucose not adjusted to stable levels despite antidiabetic therapy and/or careful medical monitoring by the family physician/specialist is not ensured), known immunodeficiency, i.e. congenital immunodeficiency, acquired immunodeficiency (e.g. HIV, some bone marrow diseases, extreme diets), iatrogenic by immunosuppressive drugs like cytostatics or by radiation therapy or immunomodulatory medications (e.g. TNF inhibitors), participation in another clinical trial with an investigational drug or device during the previous 3 months before Baseline/Visit 2, known allergy to any of the tested treatment products, a positive pregnancy test indicating pregnancy in a woman of childbearing potential at Baseline/ Visit 2, women who are pregnant or breastfeeding, pre-menopausal (last menstruation $\leq 1$ year prior to screening) sexually active women who: are of childbearing potential and are not practising an acceptable method of birth control, or do not plan to continue practising an acceptable method of birth control throughout the trial, people previously randomised in this study, history of, or current drug or alcohol abuse, psychiatric condition that might limit the participation in the study and/or that lead to the assumption that the person's ability to completely understand the consequences of consent is missing, close affiliation with the investigator (e.g. a close relative) or persons working at a study site, or person who is an employee of the sponsor's company, people who are institutionalised because of legal or regulatory order, any diseases or circumstances in which the person should not participate in the study in the opinion of the investigator

Disease duration: Unknown

Comparable at baseline: Unknown

Causative species: Dermatophytes 
EUCTR2016-001204-39 (Continued)

Number of people lost to follow-up: Unknown

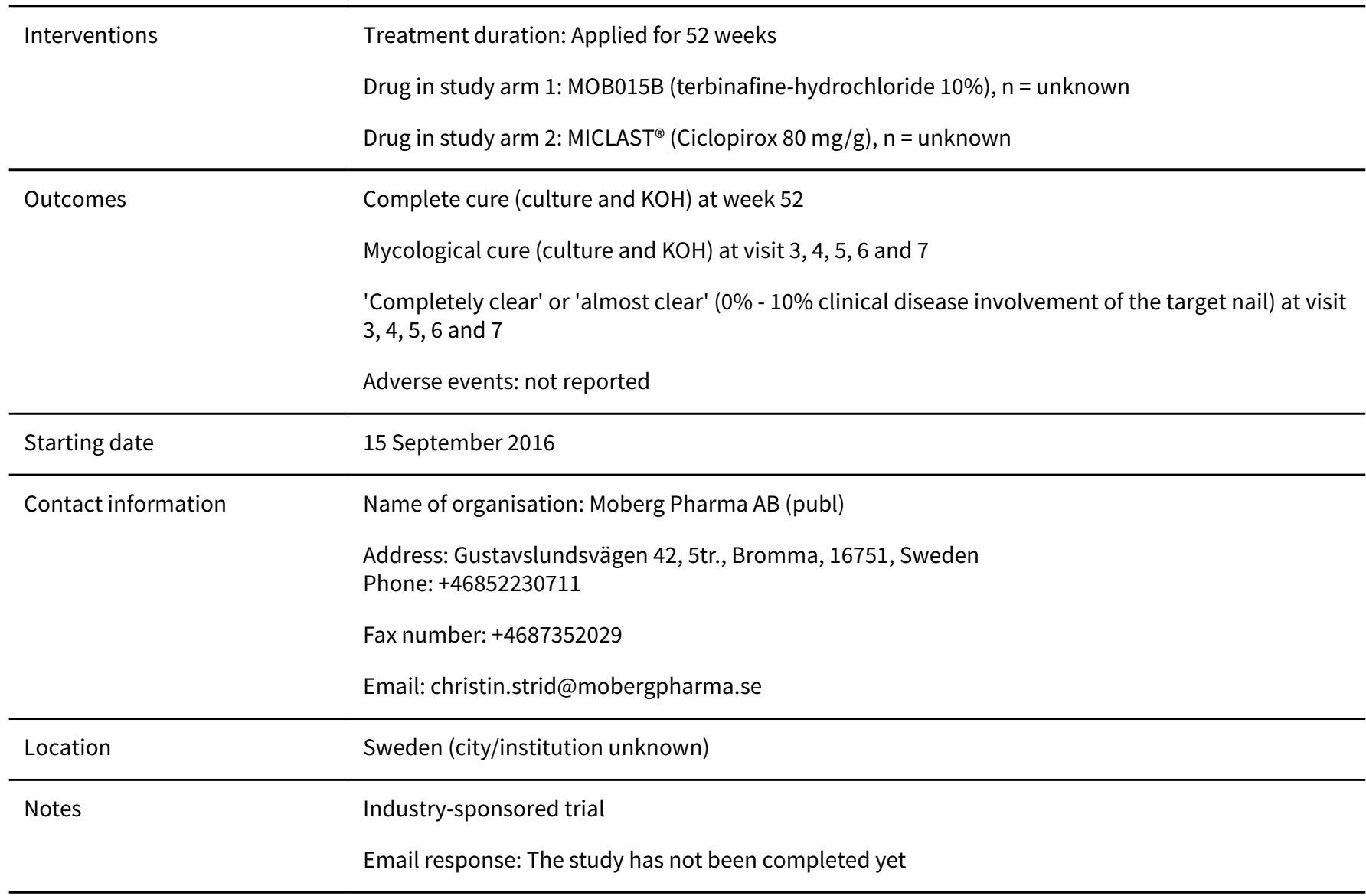

\section{EUCTR2016-003784-19}

Trial name or title

Phase III, multicentre, randomised, double-blind, parallel-group, clinical trial to evaluate the efficacy and safety of a new medicated nail lacquer for the treatment of toenail fungal infection

RCT (parallel)
Study design: Double blind
Sample size calculation: Unknown
Diagnosis mycology: KOH and culture
Big toenail specified: Yes
Statistical comparisons for outcomes: Unknown
Duration of follow-up: 52 weeks
Time points of measurements: At week 48 and 52

Participants

Total n: 360

Age: $18-70$

Sex: Men and women (ratio unknown) 
Inclusion criteria: Adults 18 - 70 years of age diagnosed with mild-to-moderate dermatophyte onychomycosis (20\% - 60\% inclusive nail involvement) of at least one big toenail with no lunula involvement. Dermatophytes confirmed with positive $\mathrm{KOH}$ and positive culture

Exclusion criteria: Allergy to medications used, life expectancy $<2$ years at screening, pregnancy or breastfeeding, unwillingness to stop nail polish, unwillingness to use contraception, systemic antifungal use 6 months prior to screening, topical antifungal use 4 weeks prior to screening, chemotherapy or immunosuppressive therapy in the 12 weeks prior to screening, systemic glucocorticoids, antimetabolites, or immunostimulants in the 4 weeks prior to screening or the need for any of these. Presence of psoriasis, uncontrolled diabetes mellitus, suspicion or evidence of severe liver or kidney disease, alcohol or substance abuse, AIDS or any other immunodeficiency. Onychomycosis caused by yeasts or non-dermatophyte moulds, mucocutaneous candidiasis, white superficial onychomycosis, proximal subungual involvement, yellow spikes on nails. Recurrent erysipela at screening, any other medical condition that the investigator determines contraindicates participation. Participation in any clinical investigation with medicine within the last 6 months prior to screening

Disease duration: Unknown

Comparable at baseline: Unknown

Causative species: Dermatophyte

Number of people lost to follow-up: Unknown

Treatment duration: 48 weeks
Drug in study arm 1: RJ-0265 cutaneous liquid, $\mathrm{n}=$ unknown
Drug in study arm 2: Ciclopirox 8\%, $\mathrm{n}=$ unknown
Drug in study arm 3: Placebo liquid, $\mathrm{n}=$ unknown

Outcomes

Complete cure at week 52

Clinical success, responder, and improvement at week 52

Negative culture at week 52

Negative $\mathrm{KOH}$ at week 52

Adverse events over 52 weeks

Growth rate of healthy nail at 52 weeks

Time in months to complete cure or clinical success

\begin{tabular}{ll}
\hline Starting date & Unknown \\
\hline Contact information & Name: Jordi Picas \\
& Email: Jordi.Picas@reigjofre.com \\
& Laboratorio Reig Jofre, SA \\
& Spain \\
\hline Location & Laboratorio Reig Jofre, SA, Sant Joan Despi, Barcelona, Spain \\
\hline Notes & Industry-sponsored trial. Study record updated 20 June 2017, status is ongoing \\
\hline
\end{tabular}




\section{JapicCTI-183840}

Trial name or title

A phase 2, multicenter, randomised, double-blind, vehicle-controlled, parallel-group study to investigate the efficacy and safety of ME1111 for 48 weeks of treatment in patients with mild-to-moderate onychomycosis

\begin{tabular}{ll}
\hline Methods & RCT (parallel) \\
Study design: Unknown & Sample size calculation: Unknown \\
& Diagnosis mycology: $\mathrm{KOH}$ and culture \\
& Big toenail specified: Yes \\
& Statistical comparisons for outcomes: Unknown \\
& Duration of follow-up: 4 weeks \\
& Time points of measurement: 52 weeks
\end{tabular}

Participants

Total n: 240

Age: 20 - 70 years old

Sex: Men and women (ratio unknown)

Inclusion criteria: Men and women, 20 to 70 years of age inclusive, diagnosed with mild-to-moderate onychomycosis of at least 1 great toenail, with $20 \%-50 \%$ nail involvement confirmed by positive fungal microscopy and culture for a dermatophyte, no more than 6 affected toenails as determined by visual inspection

Exclusion criteria: History of allergy to components of the solution product (including alcohol), underlying uncontrolled disease at discretion of investigator (e.g. cardiovascular disease, liver disease, pulmonary disease, blood disease, etc.), uncontrolled diabetes mellitus, any antifungal treatment, laser, or other therapy for onychomycosis within 24 weeks of study drug administration, unsuccessful attempts at previous antifungal treatment for onychomycosis, unwilling to refrain from topical antifungals for tinea pedis, nail or anatomic abnormalities, presence of history of psoriasis, chronic moccasin type tinea pedis, immunodeficiencies (HIV), women who are nursing, pregnant, planning pregnancy, or men with plans to have partners become pregnant

Disease duration: Unknown

Comparable at baseline: Unknown

Causative species: Dermatophyte

Number of people lost to follow-up: Unknown
Drug in study arm 1: ME1111 solution, once a day for 48 weeks

Drug in study arm 2: Vehicle solution, once a day for 48 weeks

Drug in study arm 3: Efinaconazole, once a day for 48 weeks

$\begin{array}{ll}\text { Outcomes } & \text { Mycological cure at } 12 \text { weeks } \\ \text { Adverse events: not reported }\end{array}$

Starting date

Contact information
February 2018, recruiting

Meiji Seika Pharma Co._ttd., clinical_trials@meiji.com 
JapicCTI-183840 (Continued)

Location Japan (city/institution unknown)

Notes $\quad$ Ongoing, last updated 5 December 2018

\section{NCT02644551}

Trial name or title

The efficacy of CELEXTO7 in the treatment of toenail onychomycosis: a phase 2, randomised, double-blind study

\begin{tabular}{ll}
\hline Methods & SCT (parallel) \\
Study design: Double blind \\
Sample size calculation: Unknown \\
Diagnosis mycology: KOH and culture \\
Big toenail specified: No \\
Statistical comparison for outcome: Unknown \\
Duration of follow-up: Unknown \\
Time points of measurement: At week 52
\end{tabular}

\section{Participants}

\section{Total n: 120}

Age: 18 years and older

\section{Sex: Unknown}

Inclusion criteria: Age > 18, clinically diagnosed onychomycosis of the target nail, presence of mildto-moderate onychomycosis, defined as $20 \%-50 \%$ of the area of the target nail being clinically affected, has a positive $\mathrm{KOH}$ examination from the target nails, has a positive dermatophyte culture from the target nail, written informed consent obtained, participant agreed to follow the protocol

Exclusion criteria: Presence of any disease or condition that might cause nail abnormalities or may interfere with the evaluation of the study drug, use of any systemic antifungal therapy within 4 weeks prior to the Screening visit or non-responsive to systemic antifungal therapy for onychomycosis, use of any prescription or over-the-counter topical antifungal therapy for the toenails within 4 weeks prior to the screening visit, woman who is pregnant, nursing an infant, or planning a pregnancy during the study period

Inability to understand and comply with the instructions of the study, people less than age 18, individuals with known allergy/hypersensitivity to Thuja occidentalis, Chelidonium majus, Eucalyptus citriodora, Tea tree or Thymus vulgaris

Disease duration: Unknown

Comparable at baseline: Unknown

Causative species: Dermatophyte

Number of people lost to follow-up: Unknown 
NCT02644551 (Continued)

Drug in study arm 3: Ciclopirox, $\mathrm{n}=$ unknown

\begin{tabular}{|c|c|}
\hline Outcomes & $\begin{array}{l}\text { Complete cure at week } 52 \\
\text { Mycological cure (microscopy and culture) at week } 52 \\
\text { Adverse events: not reported }\end{array}$ \\
\hline Starting date & November 2016 \\
\hline Contact information & $\begin{array}{l}\text { Principal investigator: Céline Devaux, MD, FRCPC 9305-9954 Quebec Inc } \\
\text { Study director: Guy Chamberland, M.Sc., Ph.D. 9305-9954 Quebec Inc } \\
\text { Names: Wil Lee, DPM, AACFAS } \\
\text { Address: Clinique podiatrique de Montréal Recruiting } \\
\text { Montreal, Quebec, Canada, H1X 2B3 } \\
\text { Phone: } 514 \text { 254-5000 } \\
\text { Email: drwlee@hotmail.com } \\
\text { Sponsors and Collaborators 9305-9954 Quebec Inc }\end{array}$ \\
\hline Location & Clinique podiatrique de Montréal, Montréal, Quebec, Canada \\
\hline Notes & Study record updated 26 April 2017, status is recruiting \\
\hline
\end{tabular}

Study design: Double blind

Sample size calculation: Unknown

Diagnosis mycology: $\mathrm{KOH}$ and culture

Big toenail specified: No

Statistical comparisons for outcome: Unknown

Duration of follow-up: At 6 months

Time point of measurement: Months 1, 2, 3, 4 and 6 
anti-fungal treatment within 6 months, no use of vasodilator drugs or isotretinoin within 6 months, willing to participate, and accepting the methods and risk

Exclusion criteria: People with other medical dermatologic condition associated with onychomycosis such as psoriasis, paronychia etc, people on immunosuppressive drugs, or the immunocompromised host, people with severe vascular disease, such as severe DVT, or poor capillary refill time, people with onychomycosis which involved in nail matrix, pregnancy or lactation, problems in communication or difficulty travelling to the faculty for the checkup

Disease duration: Unknown

Comparable at baseline: Unknown

Causative species: Non-dermatophyte or mixed-infection

Number of people lost to follow up: Unknown

Treatment duration: Laser had 2 passes each visit with 4-week intervals, amorolfine was applied once a week for 6 months

Drug in study arm 1: Amorolfine nail lacquer, $\mathrm{n}=$ unknown

Drug in study arm 2: Long-pulsed 1064nm, $n=$ unknown

Drug in study arm 3: Long-pulsed 1064nm and amorolfine, $\mathrm{n}=$ unknown

Outcomes Mycological cure (negative culture) at months 1, 2, 3, 4, and 6

Adverse events: not reported

\begin{tabular}{ll}
\hline Starting date & August 2016 \\
\hline Contact information & Name: Charussri Leeyaphan, MD \\
& Department of Dermatology Siriraj Hospital \\
& Bangkoknoi, Bangkok, Thailand, 10700 \\
& Phone: 6624194333 \\
& Email: charussrilee@gmail.com \\
& Sponsors and Collaborators \\
& Mahidol University \\
\hline Location & Department of Dermatology Siriraj Hospital, Bangkoknoi, Bangkok, Thailand \\
\hline Notes & $\begin{array}{l}\text { Supported by academic institution. Recruiting, estimated completion date December 2019. Last } \\
\text { update } 8 \text { March 2019 }\end{array}$ \\
\hline
\end{tabular}

\section{NCT02859519}

\section{Trial name or title}

A multi-centre, double-blind, randomised, vehicle-controlled study of efficacy and safety of topical MOB015B in the treatment of mild-to-moderate distal subungual onychomycosis (DSO)

\begin{tabular}{ll}
\hline Methods & RCT (parallel) \\
& Study design: Double blind \\
& Sample size calculation: Unknown
\end{tabular}


NCT02859519 (Continued)

Diagnosis mycology: Culture

Big toenail specified: Yes

Statistical comparison for outcomes: Unknown

Duration of follow-up: Unknown

Time points of measurements: Weeks 52
Total n: Unknown

Age: 12 - 70 years old

\section{Sex: Unknown}

Inclusion criteria: Men or women 12 - 70 years of age, DSO of at least 1 of the great toenail(s) affecting $20 \%$ to $50 \%$ of the target nail, positive culture for dermatophytes, written informed consent

Exclusion criteria: PSO, DSO of both great toenails where involvement has extended into the proximal portion of the target nail (unaffected proximal nail is $<3 \mathrm{~mm}$ ), target toenail thickness $>3 \mathrm{~mm}$, 'Spike' of onychomycosis extending to eponychium of the target toenail, presence of dermatophytoma (defined as thick masses of fungal hyphae and necrotic keratin between the nail plate and nail bed) on the target nail, other conditions than DSO known to cause abnormal nail appearance, presence of toenail infection other than dermatophytes, previous target toenail surgery with any residual disfigurement, topical treatment of the nails with other antifungal medication within 1 month before Baseline/Visit 2, systemic use of antifungal treatment within 7 months before Baseline/Visit 2, severe moccasin tinea pedis, signs of severe peripheral circulatory insufficiency, uncontrolled diabetes mellitus, known immunodeficiency, participation in another clinical trial with an investigational drug or device during the previous 3 months before Baseline/Visit 2, known allergy to any of the tested treatment products, a positive pregnancy test at Baseline/Visit 2 indicating pregnancy in a woman of childbearing potential or a premenarche woman, women who are pregnant or breastfeeding, men who have female sexual partners of child-bearing potential and sexually active women of child-bearing potential who are not practising an acceptable method of birth control, or who will not remain abstinent through the trial, people previously randomised in this study, history of, or current drug or alcohol abuse, psychiatric condition that might limit the participation in the study and/or that lead to the assumption that the person's ability to completely understand the consequences of consent is missing, close affiliation with the investigator (e.g. a close relative) or persons working at a study site, or person who is an employee of the sponsor's company, people who are institutionalised because of legal or regulatory order, any diseases or circumstances in which the person should not participate in the study in the opinion of the investigator

Disease duration: Unknown

Comparable at baseline: Unknown

Causative species: Dermatophytes

Number of people lost to follow-up: Unknown
Treatment duration: For 52 weeks

Drug in study arm 1: MOB015B, $\mathrm{n}=$ unknown

Drug in study arm 2: MOB015B Vehicle, $n=$ unknown

Outcomes Complete cure at 52 weeks

Adverse events: not reported

\begin{tabular}{ll}
\hline Starting date & October 2016 \\
\hline Contact information & Name: Kjell Rensfeldt, MD \\
\hline
\end{tabular}


Phone: +46 852230700

Email: kjell.rensfeldt@mobergpharma.se

Name: Anna Hill, MSc

Phone: +46852230700

Email: anna.hill@mobergpharma.se

USA and Canada

Sponsors and Collaborators

Moberg Pharma AB

Location

United States, multiple sites in California, Florida, Idaho, Illinois, Minnesota, New Jersey, Oregon, Texas, and Virginia

Canada, multiple locations

Cities/institutions unknown safety of topical MOB015B in the treatment of mild-to-moderate Distal Subungual Onychomycosis (DSO)

Rethods (parallel)
Study design: Single blind
Sample size calculation: Unknown
Diagnosis mycology: Culture
Big toenail specified: Yes
Statistical comparison for outcomes: Unknown
Duration of follow-up: Unknown
Time points of measurement: Weeks 52

Participants

Total n: Unknown

Age: 18 - 70 years old

Sex: Unknown

Inclusion criteria: Men or women 18 - 70 years of age, DSO of at least 1 of the great toenail(s) affecting $20 \%$ to $50 \%$ of the target nail, positive culture for dermatophytes, signed written informed consent

Exclusion criteria: PSO, DSO of both great toenails where involvement has extended into the proximal portion of the target nail (unaffected proximal nail is $<3 \mathrm{~mm}$ ), target toenail thickness more than $3 \mathrm{~mm}$, 'Spike' of onychomycosis extending to eponychium of the target toenail, presence of dermatophytoma (defined as thick masses of fungal hyphae and necrotic keratin between the nail plate and nail bed) on the target nail, other conditions than DSO known to cause abnormal nail ap- 
pearance, presence of toenail infection other than dermatophytes, previous target toenail surgery with any residual disfigurement, topical treatment of the nails with other antifungal medication or medical device within 1 month before Baseline/Visit 2, systemic use of antifungal treatment within 7 months before Baseline/Visit 2, severe moccasin tinea pedis, signs of severe peripheral circulatory insufficiency, uncontrolled diabetes mellitus, known immunodeficiency, participation in another clinical trial with an investigational drug or device during the previous 3 months before Baseline/Visit 2, known allergy to any of the tested treatment products, a positive pregnancy test indicating pregnancy in a woman of childbearing potential at Baseline/Visit 2, women who are pregnant or breastfeeding, pre-menopausal (last menstruation $\leq 1$ year prior to screening) sexually active women who are of childbearing potential and are not practising an acceptable method of birth control, or do not plan to continue practising an acceptable method of birth control throughout the trial, people previously randomised in this study

History of, or current drug or alcohol abuse, psychiatric condition that might limit the participation in the study and/or that lead to the assumption that the person's ability to completely understand the consequences of consent is missing, close affiliation with the investigator (e.g. a close relative) or persons working at a study site, or person who is an employee of the sponsor's company, people who are institutionalised because of legal or regulatory order, any diseases or circumstances in which the person should not participate in the study in the opinion of the investigator

Disease duration: Unknown

Comparable at baseline: Unknown

Causative species: Dermatophytes

Number of people lost to follow up: Unknown

\begin{tabular}{ll}
\hline Interventions & Treatment duration: For 52 weeks \\
& Drugs in study arm 1: MOB015B, $\mathrm{n}=$ unknown \\
& Drugs in study arm 2: Ciclopirox $80 \mathrm{mg} / \mathrm{g}, \mathrm{n}=$ unknown \\
\hline Outcomes & Complete cure for 52 weeks \\
& Adverse events: not reported \\
\hline Starting date & September 2016 \\
\hline Contact information & Name: Kjell Rensfeldt, MD \\
& Phone: +46852230700 \\
& Email: kjell.rensfeldt@mobergpharma.se \\
& Name: Christin Strid, MSc \\
& Phone: +4682230700 \\
& Email: christin.strid@mobergpharma.se \\
& Germany and Poland \\
& Sponsors and Collaborators \\
Moberg Pharma AB
\end{tabular}

Location Multiple locations in Germany, Poland, and the United Kingdom (cities/institutions unknown)


NCT02961634

Trial name or title

Study efficacy and safety in comparative use of investigational product adjuvant treatment in onychomycosis

\begin{tabular}{ll}
\hline Methods & RCT (parallel) \\
Study design: Single blind \\
Sample size calculation: Unknown \\
Diagnosis mycology: Culture \\
Big toenail specified: No \\
Statistical comparisons for outcome: Unknown \\
Duration of follow-up: Unknown \\
Timr points for measurement: At days 45 and 90 \\
\hline
\end{tabular}

\section{Participants}

Total $\mathrm{n}: 46$

Age: 18 - 65 years old

Sex: Unknown

Inclusion criteria: Volunteers of both sexes, aged 18 - 65 years, with onychomycosis confirmed by positive direct mycological nail of hands or feet, agreement to comply with the test procedures and attend the clinic on the days and times for certain applications or assessments or both, understand, consent and sign the "Instrument of Consent of Clarified"

Exclusion criteria: Pregnancy or risk pregnancy/lactation, use of anti-inflammatory/immunosuppressive drugs (in the last 30 days and during the study), concomitant nail pathologies (psoriasis, lichen planus, etc.), systemic conditions that may compromise the growth of the nail (vascular disease, diabetes, etc.), irritation. History of similar products to the investigational product, treatment with antifungal medication prior to the study (up to 12 weeks for systemic medications and topical medications for 4 weeks), other conditions considered by the investigator physician as reasonable for the disqualification of the individual's participation in the study

Disease duration: Unknown

Comaprable at baseline: Unknown

Causative species: Unknown

Number of people lost to follow-up: Unknown

Interventions

Treatment duration: Twice daily, morning and night for 30 days, ciclopirox every other day for 1 month, twice a week for a month and once a week for a month Drug in study arm 1: Nailner 2 in 1 and Ciclopirox 8\%, $\mathrm{n}=$ unknown

Drug in study arm 2: Ciclopirox 8\%, $\mathrm{n}=$ unknown

Outcomes

Mycological cure (culture) at days 45 and 90

Adverse events: not reported

\begin{tabular}{ll}
\hline Starting date & April 2017 \\
\hline Contact information & Name: Ana Carolina Prazias Massei \\
& Phone: $+55(16) 3624-4056$ \\
& Email: carolina.prazias@mipbrasilfarma.com.br
\end{tabular}


NCT02961634 (Continued)

\section{Sponsors and Collaborators}

MIP Brasil Indústria e Comércio de Produtos Farmacêuticos LTDA

Buranello e Rodrigues Consultoria em Desenvolvimento Farmacêutico Ltda ME

\begin{tabular}{ll}
\hline Location & Unknown \\
\hline Notes & $\begin{array}{l}\text { Industry provided financial support. Study record updated 31 March 2017, status is not yet recruit- } \\
\text { ing }\end{array}$ \\
\hline
\end{tabular}

\section{NCT03098342}

\section{Trial name or title}

Comparison of efficacy and safety between methylene blue-mediated photodynamic therapy and $5 \%$ amorolfine nail lacquer for toenail onychomycosis treatment in Asians

\section{Methods}

\section{RCT (parallel)}

Study design: Single blind

Sample size calculation: Unknown

Diagnosis mycology: Culture, $\mathrm{KOH}, \mathrm{PAS}$ and/or GMS

Big toenail specified: Unknown

Statistical comparisons for outcomes: Unknown

Duration of follow-up: 18 months.

Time points of measurements: At weeks 2, 4, 6, 8, 10, 12, 20, 28, 36, 44, 52, 60, 68, 76

Age: 18 - 90 years old

\section{Sex: Unknown}

Inclusion criteria: Adults, aged between 18 and 90 years, with distal and lateral subungual toenail onychomycosis diagnosed clinically and mycologically clinical signs of onychomycosis as following: discolouration, dystrophy of nail plate, subungual hyperkeratosis

Onycholysis confirmed by: positive culture of dermatophyte or non-dermatophyte (must be isolated from sequential specimens) and/or positive microscopic evidence, any of these methods, direct microscopy (KOH preparation), nail plate culture on Sabouraud's dextrose agar (SDA), histopathological examination of nail clippings using PAS staining (HPE-PAS), histopathological examination of nail clippings using GMS staining (HPE-GMS), polymerase chain reaction

Exclusion criteria: Those with nail changes because of skin disease or associated systemic diseases, pregnancy or lactating women, those who are allergic to amorolfine, methylene blue, those who are photosensitive to visible light, those who had previously used anti-fungal medications within 3 months for systemic and 1 month for topical therapy

Disease duration: Unknown

Comparable at baseline: Unknown

Causative species: Dermatophyte

Number of people lost to follow-up: Unknown 
NCT03098342 (Continued)

Interventions
Treatment duration: 6 treatments, 15 days between each sessions or lacquer applied daily

Drug in study arm 1: Methylene blue-mediated photodynamic therapy, $\mathrm{n}=$ unknown

Drug in study arm 2: Amorolfine 5\% nail lacquer, $n=$ unknown

\section{Outcomes}

Onychomycosis severity index (OSI) change from baseline at weeks 2, 4, 6, 8, 10, 12, 20, 28, 36, 44, $52,60,68$, and 76

Adverse events: not reported

\begin{tabular}{ll}
\hline Starting date & 1 June 2017 \\
\hline Contact information & Name: Nutthamon Bowornsathitchai, M.D. \\
& Phone: 66818239488 \\
& Email: bee_o_bo@hotmail.com \\
& King Chulalongkorn Memorial Hospital \\
& Bangkok, Thailand, 10330 \\
& Sponsors and Collaborators \\
& Chulalongkorn University, King Chulalongkorn Memorial Hospital \\
\hline Location & King Chulalongkorn Memorial Hospital \\
\hline Notes & Bangkok, Thailand \\
\hline
\end{tabular}

\section{NCT03814343}

Trial name or title

Comparison of effectiveness of topical amphotericin B in 30\% dimethylsulphoxide (DMSO) and $30 \%$ DMSO in treating non-dermatophytes onychomycosis: Randomised double-blind controlled trial pilot study

\begin{tabular}{ll}
\hline Methods & Study design: Double blind \\
Sample size calculation: No \\
Diagnosis mycology: Culture \\
Big toenail specified: Unknown \\
Statistical comparisons for outcomes: Unknown \\
Duration of follow-up: 36 weeks \\
Time points of measurements: At week 36
\end{tabular}

Participants

Total $\mathrm{n}: 20$

Age: 18 years and older.

Sex: Men and women (ratio unknown) 
NCT03814343 (Continued)

Inclusion criteria: Adults 18 years of age and older diagnosed with non-dermatophyte onychomycosis and have not been treated with any oral/IV/topical antifungal therapy within 36 weeks before enrolled

Exclusion criteria: People with dermatophyte onychomycosis, immunocompromisation, or concomitant nail diseases

Disease duration: Unknown

Comparable at baseline: Unknown

Causative species: Non-dermatophytes

Number of people lost to follow-up: Unknown

\begin{tabular}{ll}
\hline Interventions & Treatment duration: 12 weeks \\
& Drug in study arm 1: Amphotericin B in 30\% DMSO, $\mathrm{n}=10$ \\
& Drug in study arm 2: Placebo ( $30 \%$ DMSO), $\mathrm{n}=10$ \\
\hline
\end{tabular}

Mycological cure at 36 weeks
Clinical cure (complete clinical improvement or $<10 \%$ nail involvement) at 36 weeks
Adverse events over 12 weeks
Median time to mycological and clinical cure

\begin{tabular}{ll}
\hline Starting date & 15 January 2019 \\
\hline Contact information & Name: Dr. Charussri Leeyaphan, M.D. \\
& Email: charussrilee@gmail.com \\
& Department of Dermatology, Siriraj Hospital \\
& Bangkoknoi, Bangkok, Thailand \\
\hline Location & Department of Dermatology, Siriraj Hospital \\
& Bangkoknoi, Bangkok, Thailand \\
\hline Notes & Study record updated 29 January 2019, status is recruiting \\
\hline
\end{tabular}

BMI: body mass index; DSO: distal subungual onychomycosis; GMS: $\mathrm{KOH}$ : potassium hydroxide; PAS: periodic acid-Schiff; PCR: polymerase chain reaction; PSO: proximal subungual onychomycosis; RCT: randomised controlled trial.

\section{DATA AND ANALYSES}

\section{Comparison 1. Ciclopirox $8 \%$ lacquer vs vehicle}

\begin{tabular}{lllll}
\hline Outcome or subgroup title & $\begin{array}{l}\text { No. of } \\
\text { studies }\end{array}$ & $\begin{array}{l}\text { No. of } \\
\text { partici- } \\
\text { pants }\end{array}$ & Statistical method & Effect size \\
\hline 1 Complete cure: $36-52$ weeks & 2 & 460 & Risk Ratio (M-H, Random, 95\% Cl) & $9.29[1.72,50.14]$ \\
\hline
\end{tabular}




\begin{tabular}{lllll}
\hline Outcome or subgroup title & $\begin{array}{l}\text { No. of } \\
\text { studies }\end{array}$ & $\begin{array}{l}\text { No. of } \\
\text { partici- } \\
\text { pants }\end{array}$ & Statistical method & Effect size \\
\hline $\begin{array}{l}\text { 2 Adverse events (directly related to } \\
\text { treatment) }\end{array}$ & 2 & 460 & Risk Ratio (M-H, Random, 95\% Cl) & $1.61[0.89,2.92]$ \\
\hline 3 Mycological cure: $36-52$ weeks & 2 & 460 & Risk Ratio (M-H, Random, 95\% Cl) & $3.15[1.93,5.12]$ \\
\hline
\end{tabular}

Analysis 1.1. Comparison 1 Ciclopirox 8\% lacquer vs vehicle, Outcome 1 Complete cure: 36 - 52 weeks.

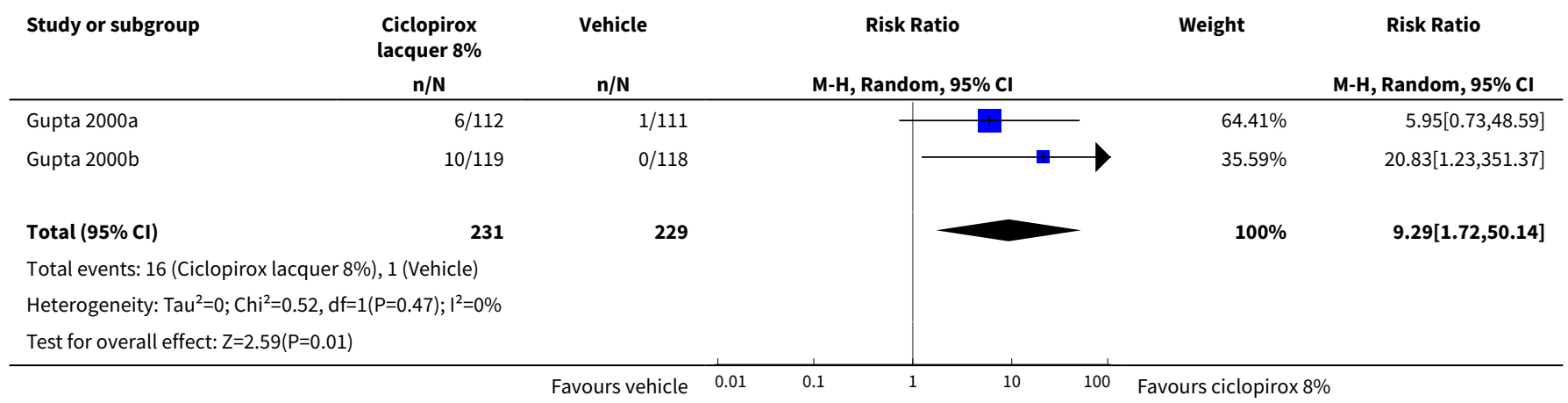

Analysis 1.2. Comparison 1 Ciclopirox $8 \%$ lacquer vs vehicle, Outcome 2 Adverse events (directly related to treatment).

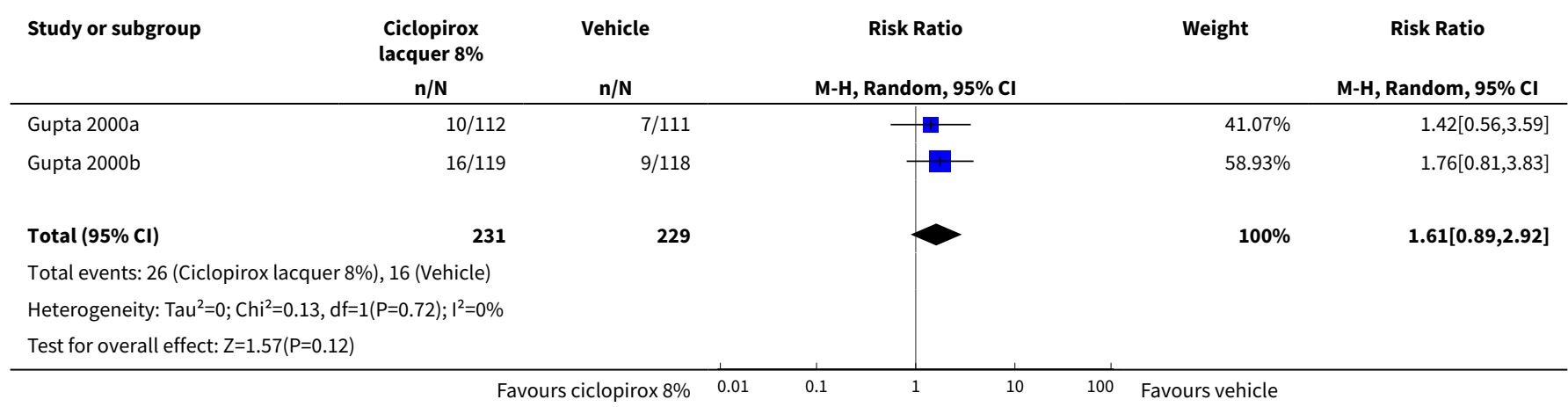

Analysis 1.3. Comparison 1 Ciclopirox $8 \%$ lacquer vs vehicle, Outcome 3 Mycological cure: 36 - 52 weeks.

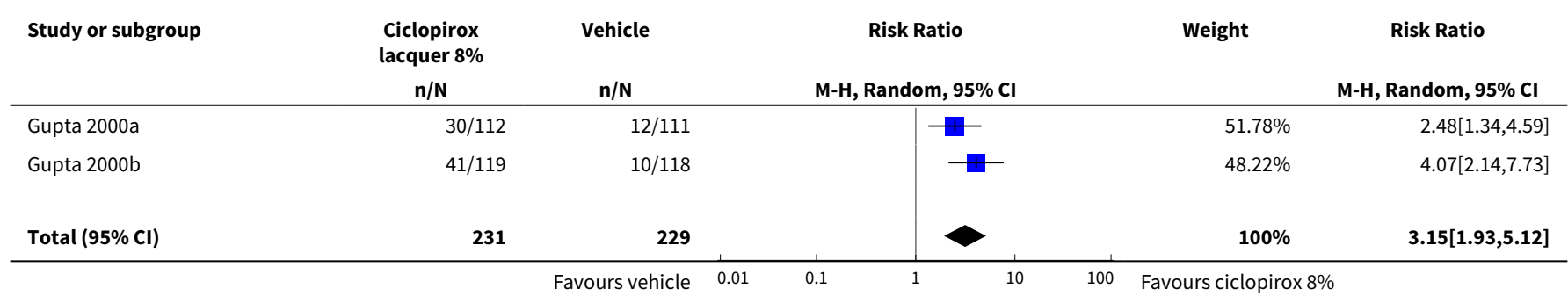




\begin{tabular}{|c|c|c|c|c|c|}
\hline \multirow[t]{2}{*}{ Study or subgroup } & \multirow{2}{*}{$\begin{array}{c}\text { Ciclopirox } \\
\text { lacquer } 8 \% \\
\mathbf{n} / \mathbf{N} \\
\end{array}$} & \multirow{2}{*}{$\begin{array}{c}\text { Vehicle } \\
\text { n/N }\end{array}$} & Risk Ratio & \multirow[t]{2}{*}{ Weight } & \multirow{2}{*}{$\begin{array}{c}\text { Risk Ratio } \\
\text { M-H, Random, 95\% CI }\end{array}$} \\
\hline & & & M-H, Random, 95\% Cl & & \\
\hline \multicolumn{6}{|c|}{ Total events: 71 (Ciclopirox lacquer 8\%), 22 (Vehicle) } \\
\hline \multicolumn{6}{|c|}{ Heterogeneity: $\operatorname{Tau}^{2}=0.02 ; \mathrm{Chi}^{2}=1.2, \mathrm{df}=1(\mathrm{P}=0.27) ; \mathrm{I}^{2}=16.38 \%$} \\
\hline \multicolumn{6}{|c|}{ Test for overall effect: $Z=4.62(P<0.0001)$} \\
\hline
\end{tabular}

\section{Comparison 2. Efinaconazole $10 \%$ solution vs vehicle}

\begin{tabular}{llllll}
\hline Outcome or subgroup title & $\begin{array}{l}\text { No. of } \\
\text { studies }\end{array}$ & $\begin{array}{l}\text { No. of } \\
\text { partici- } \\
\text { pants }\end{array}$ & Statistical method & Effect size \\
\hline 1 Complete cure: 40 - 52 weeks & 3 & 1716 & Risk Ratio (M-H, Random, 95\% Cl) & $3.54[2.24,5.60]$ \\
\hline 2 Adverse events & 3 & 1701 & Risk Ratio (M-H, Random, 95\% Cl) & $1.10[1.01,1.20]$ \\
\hline 3 Mycological cure: 40 - 52 weeks & 3 & 1716 & Risk Ratio (M-H, Random, 95\% Cl) & $2.31[1.08,4.94]$ \\
\hline 4 Clinical cure: 40 - 52 weeks & 2 & 1655 & Risk Ratio (M-H, Random, 95\% Cl) & $3.07[2.08,4.53]$ \\
\hline
\end{tabular}

Analysis 2.1. Comparison 2 Efinaconazole 10\% solution vs vehicle, Outcome 1 Complete cure: 40 - 52 weeks.

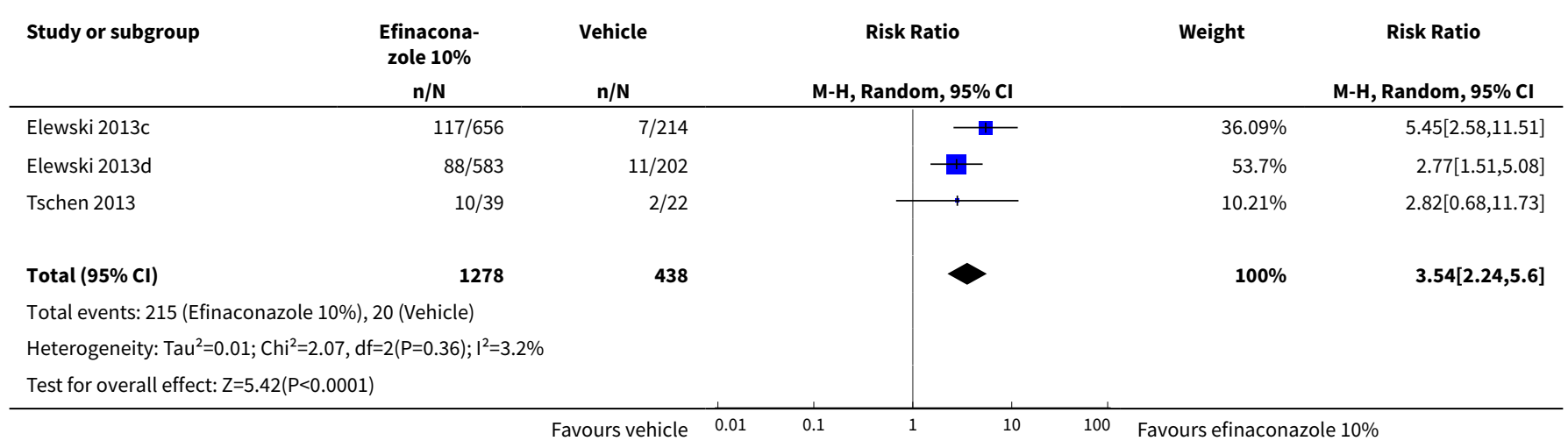

Analysis 2.2. Comparison 2 Efinaconazole $10 \%$ solution vs vehicle, Outcome 2 Adverse events.

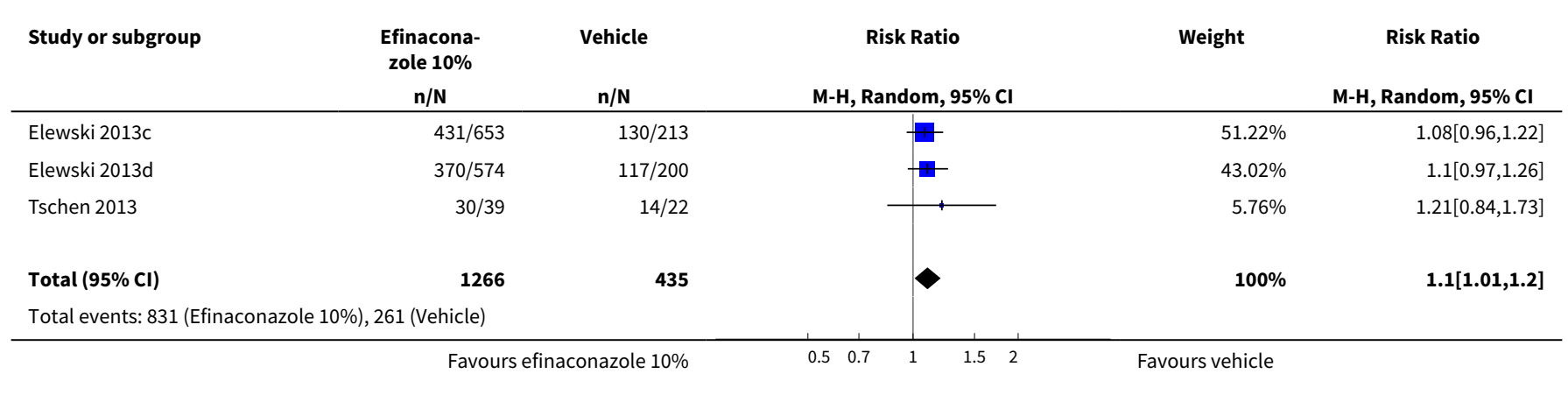




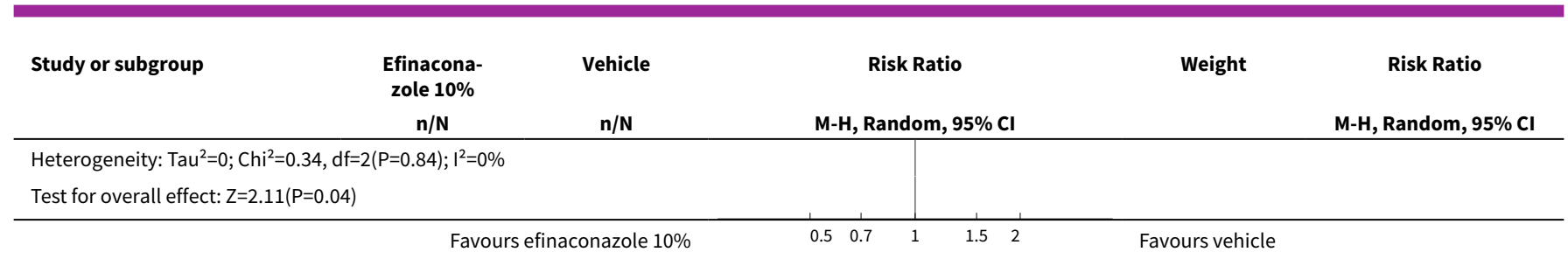

Analysis 2.3. Comparison 2 Efinaconazole 10\% solution vs vehicle, Outcome 3 Mycological cure: 40 - 52 weeks.

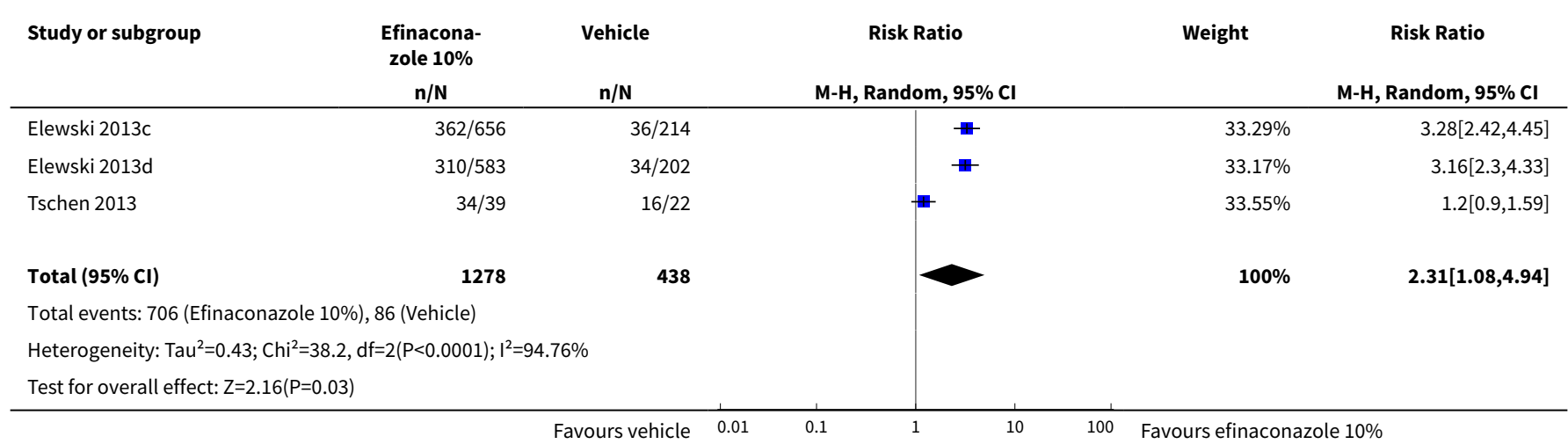

Analysis 2.4. Comparison 2 Efinaconazole $10 \%$ solution vs vehicle, Outcome 4 Clinical cure: 40 - 52 weeks.

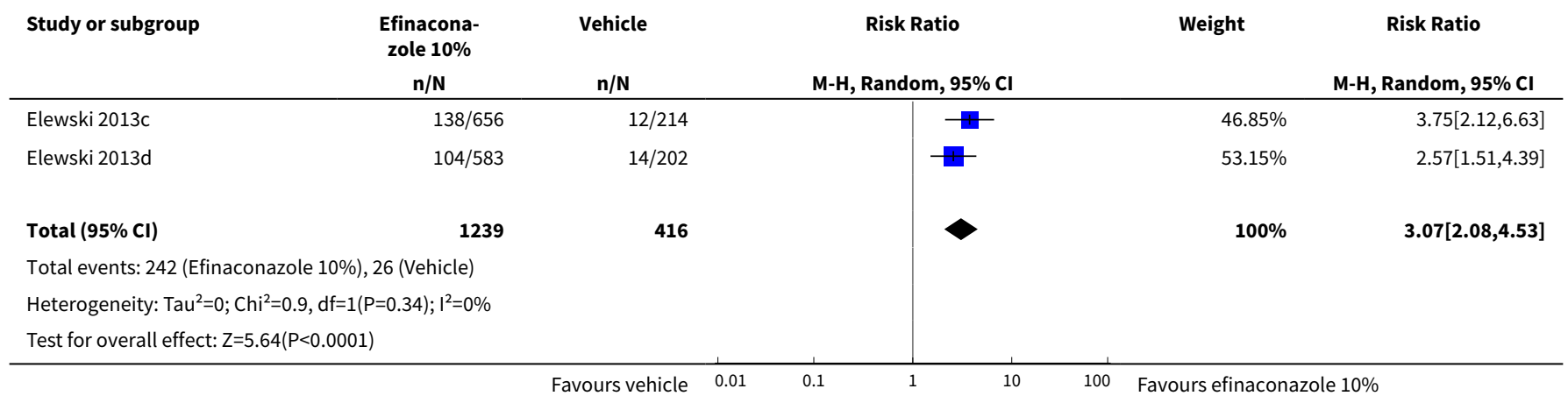

\section{Comparison 3. Tavaborole $5 \%$ solution vs vehicle}

\begin{tabular}{lllll}
\hline Outcome or subgroup title & $\begin{array}{l}\text { No. of } \\
\text { studies }\end{array}$ & $\begin{array}{l}\text { No. of } \\
\text { partici- } \\
\text { pants }\end{array}$ & Statistical method & Effect size \\
\hline 1 Complete cure: $36-52$ weeks & 2 & 1198 & Risk Ratio (M-H, Random, 95\% Cl) & $7.40[2.71,20.24]$ \\
\hline 2 Adverse events & 2 & 1186 & Risk Ratio (M-H, Random, 95\% Cl) & $0.98[0.85,1.13]$ \\
\hline $\begin{array}{l}\text { 3 Adverse events (directly related } \\
\text { to treatment) }\end{array}$ & 2 & 1186 & Risk Ratio (M-H, Random, 95\% Cl) & $3.82[1.65,8.85]$ \\
\hline
\end{tabular}




\begin{tabular}{lllll}
\hline Outcome or subgroup title & $\begin{array}{l}\text { No. of } \\
\text { studies }\end{array}$ & $\begin{array}{l}\text { No. of } \\
\text { partici- } \\
\text { pants }\end{array}$ & Statistical method & Effect size \\
\hline 4 Mycological cure: $36-52$ weeks & 2 & 1198 & Risk Ratio (M-H, Random, 95\% Cl) & $3.40[2.34,4.93]$ \\
\hline
\end{tabular}

Analysis 3.1. Comparison 3 Tavaborole $5 \%$ solution vs vehicle, Outcome 1 Complete cure: $\mathbf{3 6}$ - 52 weeks.

\begin{tabular}{|c|c|c|c|c|c|}
\hline Study or subgroup & $\begin{array}{l}\text { Tavaborole } \\
\mathbf{5 \%} \text { solution }\end{array}$ & Vehicle & $\begin{array}{c}\text { Risk Ratio } \\
\text { M-H, Random, 95\% Cl }\end{array}$ & Weight & $\begin{array}{c}\text { Risk Ratio } \\
\text { M-H, Random, } 95 \% \mathrm{CI}\end{array}$ \\
\hline Elewski 2015a & $26 / 400$ & $1 / 194$ & $\longrightarrow$ & $25.55 \%$ & $12.61[1.72,92.24]$ \\
\hline Elewski 2015b & $36 / 399$ & $3 / 205$ & $\longrightarrow$ & $74.45 \%$ & $6.17[1.92,19.78]$ \\
\hline Total $(95 \% \mathrm{Cl})$ & 799 & 399 & & $100 \%$ & $7.4[2.71,20.24]$ \\
\hline \multicolumn{6}{|c|}{ Total events: 62 (Tavaborole 5\% solution), 4 (Vehicle) } \\
\hline \multicolumn{6}{|c|}{ Heterogeneity: $\operatorname{Tau}^{2}=0 ; \mathrm{Chi}^{2}=0.38, \mathrm{df}=1(\mathrm{P}=0.54) ; \mathrm{I}^{2}=0 \%$} \\
\hline \multicolumn{6}{|c|}{ Test for overall effect: $Z=3.9(P<0.0001)$} \\
\hline
\end{tabular}

Analysis 3.2. Comparison 3 Tavaborole $5 \%$ solution vs vehicle, Outcome 2 Adverse events.

\begin{tabular}{|c|c|c|c|c|c|}
\hline Study or subgroup & $\begin{array}{c}\text { Tavaborole } \\
5 \% \text { solution } \\
\mathrm{n} / \mathrm{N} \\
\end{array}$ & $\begin{array}{c}\text { Vehicle } \\
\mathrm{n} / \mathrm{N} \\
\end{array}$ & $\begin{array}{c}\text { Risk Ratio } \\
\text { M-H, Random, } 95 \% \mathrm{Cl}\end{array}$ & Weight & $\begin{array}{c}\text { Risk Ratio } \\
\text { M-H, Random, } 95 \% \mathrm{Cl}\end{array}$ \\
\hline Elewski 2015a & $255 / 396$ & $135 / 193$ & -1 & $55.64 \%$ & $0.92[0.82,1.04]$ \\
\hline Elewski 2015b & $227 / 395$ & $109 / 202$ & - & $44.36 \%$ & $1.07[0.91,1.24]$ \\
\hline Total $(95 \% \mathrm{Cl})$ & 791 & 395 & & $100 \%$ & $0.98[0.85,1.13]$ \\
\hline \multicolumn{6}{|c|}{ Total events: 482 (Tavaborole 5\% solution), 244 (Vehicle) } \\
\hline \multicolumn{6}{|c|}{ Heterogeneity: $\operatorname{Tau}^{2}=0.01 ; \mathrm{Chi}^{2}=2.26, \mathrm{df}=1(\mathrm{P}=0.13) ; 1^{2}=55.7 \%$} \\
\hline \multicolumn{6}{|c|}{ Test for overall effect: $Z=0.25(P=0.81)$} \\
\hline
\end{tabular}

\section{Analysis 3.3. Comparison 3 Tavaborole $5 \%$ solution vs vehicle, Outcome 3 Adverse events (directly related to treatment).}

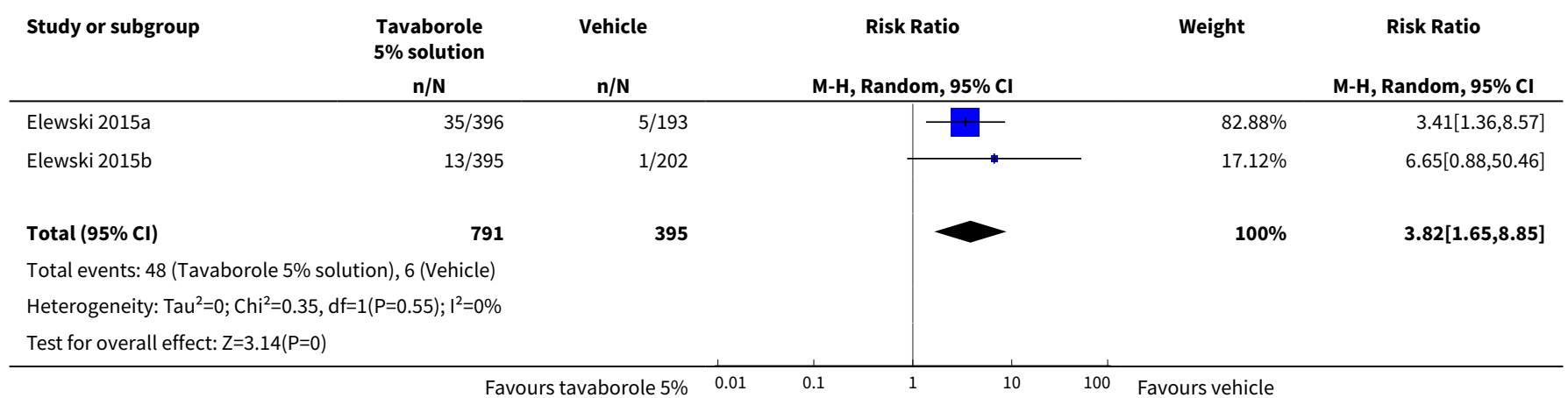


Analysis 3.4. Comparison 3 Tavaborole $5 \%$ solution vs vehicle, Outcome 4 Mycological cure: 36 - 52 weeks.

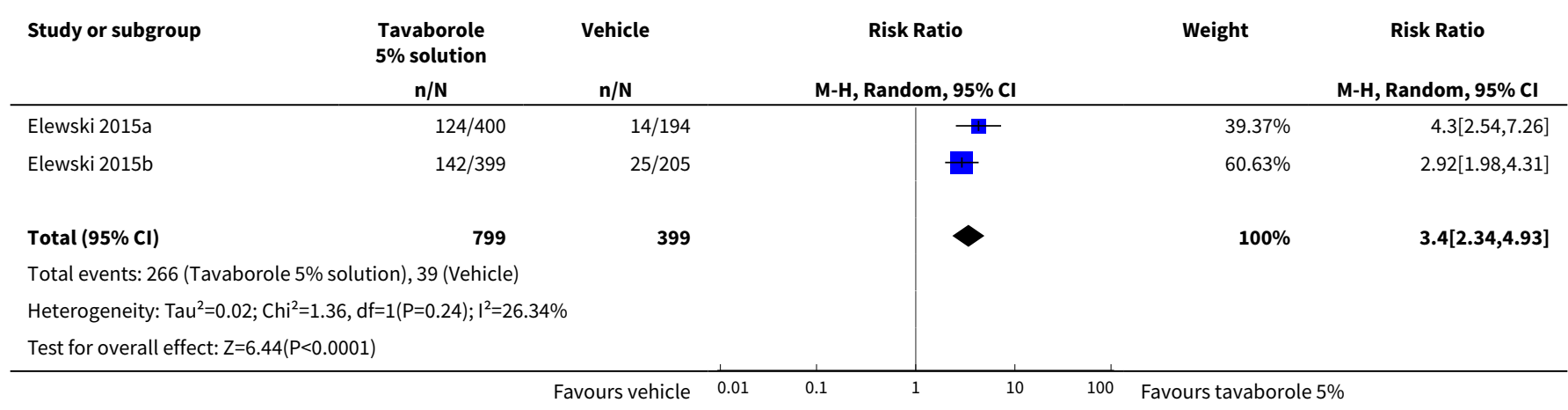

Comparison 4. P-3051 (ciclopirox $8 \%$ hydrolacquer) vs comparators

\begin{tabular}{|c|c|c|c|c|}
\hline Outcome or subgroup title & $\begin{array}{l}\text { No. of } \\
\text { studies }\end{array}$ & $\begin{array}{l}\text { No. of } \\
\text { partici- } \\
\text { pants }\end{array}$ & Statistical method & Effect size \\
\hline 1 Complete cure: 48 - 52 weeks & 2 & 490 & Risk Ratio (M-H, Random, 95\% Cl) & $2.43[1.32,4.48]$ \\
\hline 2 Adverse events & 2 & 487 & Risk Ratio (M-H, Random, 95\% Cl) & $0.60[0.19,1.92]$ \\
\hline 3 Mycological cure: 48 - 52 weeks & 2 & 490 & Risk Ratio (M-H, Random, 95\% Cl) & $1.08[0.85,1.37]$ \\
\hline $\begin{array}{l}4 \text { Mycological cure (participants with } \\
\text { dermatophyte infections): } 48 \text { - } 52 \\
\text { weeks }\end{array}$ & 2 & 460 & Risk Ratio (M-H, Random, 95\% Cl) & $1.07[0.85,1.33]$ \\
\hline
\end{tabular}

Analysis 4.1. Comparison 4 P-3051 (ciclopirox $8 \%$ hydrolacquer) vs comparators, Outcome 1 Complete cure: 48 - 52 weeks.

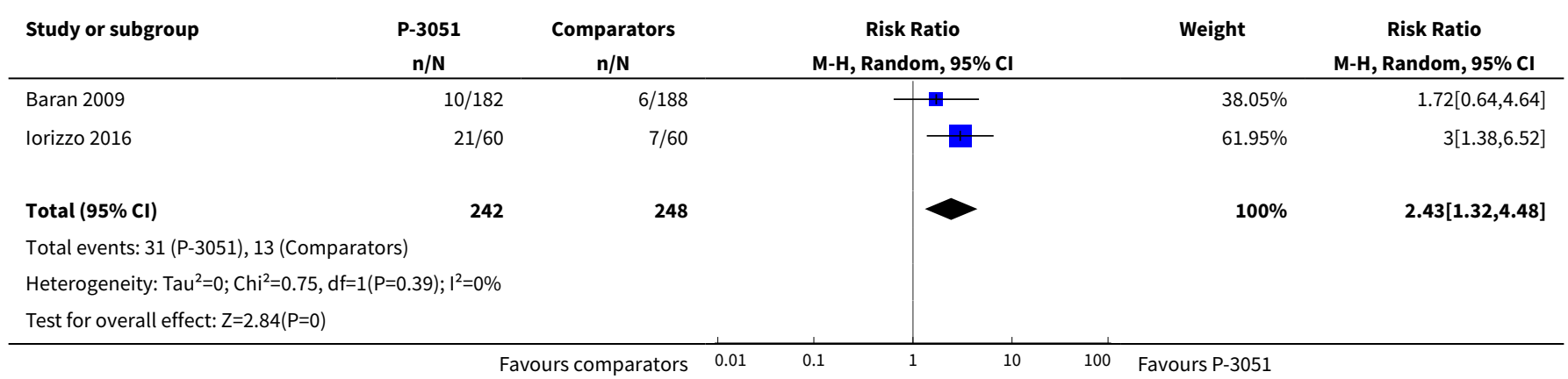


Analysis 4.2. Comparison 4 P-3051 (ciclopirox 8\% hydrolacquer) vs comparators, Outcome 2 Adverse events.

\begin{tabular}{|c|c|c|c|c|c|}
\hline Study or subgroup & $\begin{array}{c}\text { P-3051 } \\
n / N\end{array}$ & $\begin{array}{c}\text { Comparators } \\
n / N\end{array}$ & $\begin{array}{c}\text { Risk Ratio } \\
\text { M-H, Random, } 95 \% \mathrm{CI} \\
\end{array}$ & Weight & $\begin{array}{c}\text { Risk Ratio } \\
\text { M-H, Random, } 95 \% \mathrm{CI}\end{array}$ \\
\hline Baran 2009 & $14 / 180$ & $30 / 187$ & - & $88.09 \%$ & $0.48[0.27,0.88]$ \\
\hline Iorizzo 2016 & $1 / 60$ & $0 / 60$ & $\longrightarrow$ & $11.91 \%$ & $3[0.12,72.2]$ \\
\hline Total $(95 \% \mathrm{Cl})$ & 240 & 247 & & $100 \%$ & $0.6[0.19,1.92]$ \\
\hline \multicolumn{6}{|c|}{ Total events: 15 (P-3051), 30 (Comparators) } \\
\hline Test for overall effect & & & & & \\
\hline
\end{tabular}

Analysis 4.3. Comparison 4 P-3051 (ciclopirox $8 \%$ hydrolacquer) vs comparators, Outcome 3 Mycological cure: 48 - 52 weeks.

\begin{tabular}{|c|c|c|c|c|c|}
\hline Study or subgroup & $\begin{array}{c}\text { P-3051 } \\
n / N\end{array}$ & $\begin{array}{c}\text { Comparators } \\
n / N\end{array}$ & $\begin{array}{c}\text { Risk Ratio } \\
\text { M-H, Random, } 95 \% \mathrm{Cl}\end{array}$ & Weight & $\begin{array}{c}\text { Risk Ratio } \\
\text { M-H, Random, } 95 \% \mathrm{CI}\end{array}$ \\
\hline Baran 2009 & $156 / 182$ & $168 / 188$ & 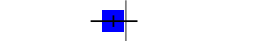 & $52.03 \%$ & $0.96[0.89,1.04]$ \\
\hline Iorizzo 2016 & $60 / 60$ & $49 / 60$ & - & $47.97 \%$ & $1.22[1.08,1.38]$ \\
\hline Total $(95 \% \mathrm{Cl})$ & 242 & 248 & & $100 \%$ & $1.08[0.85,1.37]$ \\
\hline \multicolumn{6}{|c|}{ Total events: 216 (P-3051), 217 (Comparators) } \\
\hline \multicolumn{6}{|c|}{ Test for overall effect: $\mathrm{Z}=0.61(\mathrm{P}=0.54)$} \\
\hline
\end{tabular}

Analysis 4.4. Comparison 4 P-3051 (ciclopirox $8 \%$ hydrolacquer) vs comparators, Outcome 4 Mycological cure (participants with dermatophyte infections): 48 - 52 weeks.

\begin{tabular}{|c|c|c|c|c|c|}
\hline Study or subgroup & $\begin{array}{c}\mathrm{P}-3051 \\
\mathrm{n} / \mathrm{N}\end{array}$ & $\begin{array}{c}\text { Comparators } \\
n / N\end{array}$ & $\begin{array}{c}\text { Risk Ratio } \\
\text { M-H, Random, 95\% Cl }\end{array}$ & Weight & $\begin{array}{c}\text { Risk Ratio } \\
\text { M-H, Random, } 95 \% \mathrm{Cl}\end{array}$ \\
\hline Baran 2009 & $156 / 182$ & $168 / 188$ & \pm & $53.12 \%$ & $0.96[0.89,1.04]$ \\
\hline Iorizzo 2016 & $43 / 43$ & $39 / 47$ & $\mathbf{H}$ & $46.88 \%$ & $1.2[1.05,1.38]$ \\
\hline Total $(95 \% \mathrm{CI})$ & 225 & 235 & & $100 \%$ & $1.07[0.85,1.33]$ \\
\hline \multicolumn{6}{|c|}{ Total events: 199 (P-3051), 207 (Comparators) } \\
\hline \multicolumn{6}{|c|}{ Test for overall effect: $Z=0.57(P=0.57)$} \\
\hline & & s comparators & 0.1 & urs P-3051 & \\
\hline
\end{tabular}

Comparison 5. 1064-nm Nd:YAG laser vs no treatment/sham

\begin{tabular}{lllll}
\hline Outcome or subgroup title & $\begin{array}{l}\text { No. of } \\
\text { studies }\end{array}$ & $\begin{array}{l}\text { No. of } \\
\text { partici- } \\
\text { pants }\end{array}$ & Statistical method & Effect size \\
\hline 1 Adverse events & 2 & 85 & Risk Ratio (M-H, Random, 95\% Cl) & $4.85[0.24,97.11]$ \\
\hline
\end{tabular}




\begin{tabular}{llllll}
\hline Outcome or subgroup title & $\begin{array}{l}\text { No. of } \\
\text { studies }\end{array}$ & $\begin{array}{l}\text { No. of } \\
\text { partici- } \\
\text { pants }\end{array}$ & Statistical method & Effect size \\
\hline 2 Mycological cure: 12 weeks & 1 & & Risk Ratio (M-H, Random, 95\% Cl) & Totals not selected \\
\hline 3 Mycological cure: $36-52$ weeks & 2 & 85 & Risk Ratio (M-H, Random, 95\% Cl) & $1.04[0.59,1.85]$ \\
\hline
\end{tabular}

Analysis 5.1. Comparison 5 1064-nm Nd:YAG laser vs no treatment/sham, Outcome 1 Adverse events.

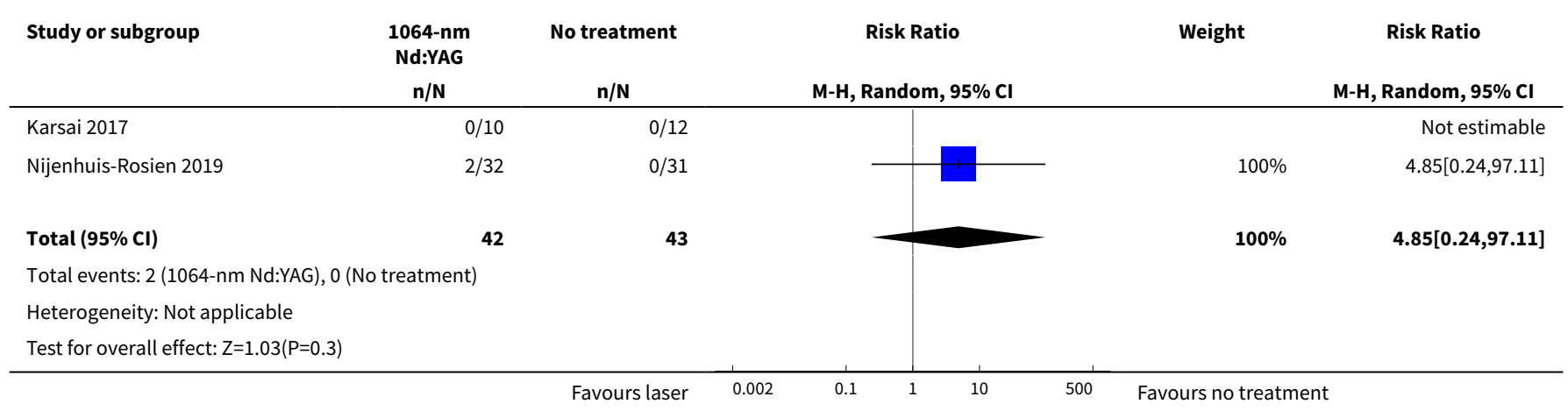

Analysis 5.2. Comparison 5 1064-nm Nd:YAG laser vs no treatment/sham, Outcome 2 Mycological cure: 12 weeks.

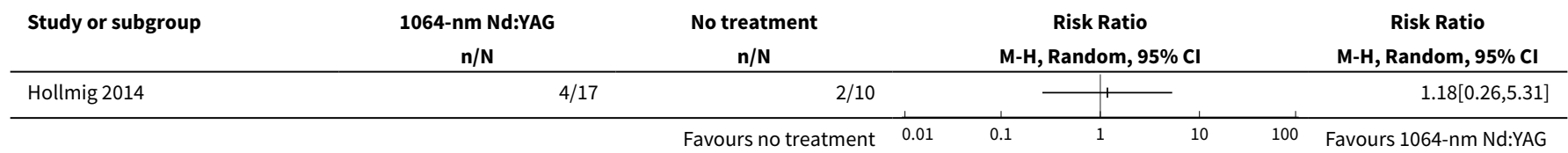

Analysis 5.3. Comparison 5 1064-nm Nd:YAG laser vs no treatment/sham, Outcome 3 Mycological cure: 36 - 52 weeks.

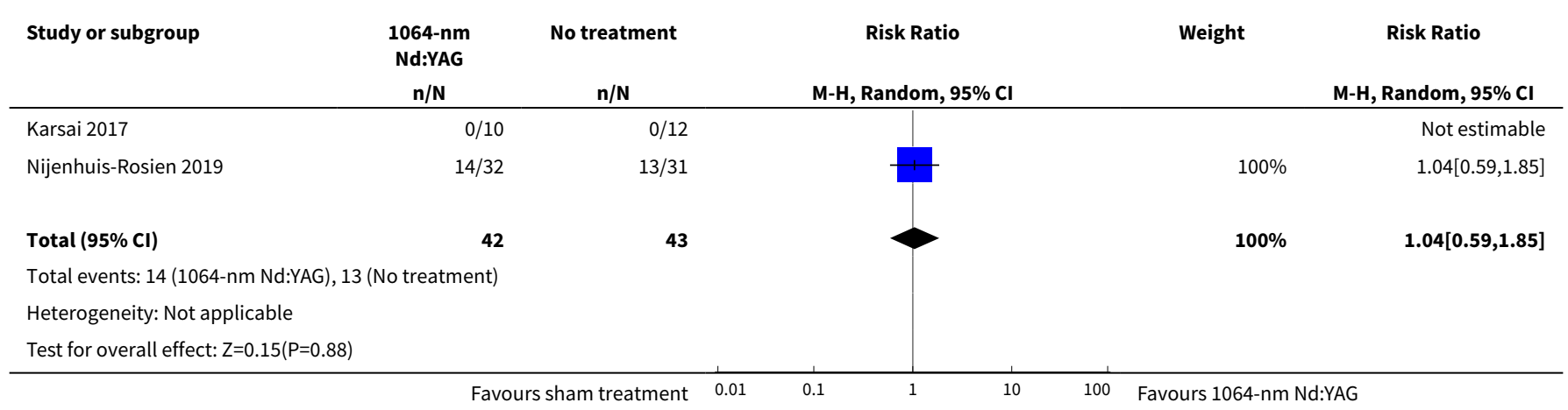


Comparison 6 . Luliconazole $5 \%$ solution vs vehicle

\begin{tabular}{lllll}
\hline Outcome or subgroup title & $\begin{array}{l}\text { No. of } \\
\text { studies }\end{array}$ & $\begin{array}{l}\text { No. of } \\
\text { partici- } \\
\text { pants }\end{array}$ & Statistical method & Effect size \\
\hline 1 Complete cure: 48 weeks & 1 & Risk Ratio (M-H, Random, 95\% Cl) & Totals not selected \\
\hline 2 Adverse events & 1 & Risk Ratio (M-H, Random, 95\% Cl) & Totals not selected \\
\hline 3 Mycological cure: 48 weeks & 1 & Risk Ratio (M-H, Random, 95\% Cl) & Totals not selected \\
\hline
\end{tabular}

Analysis 6.1. Comparison 6 Luliconazole $5 \%$ solution vs vehicle, Outcome 1 Complete cure: 48 weeks.

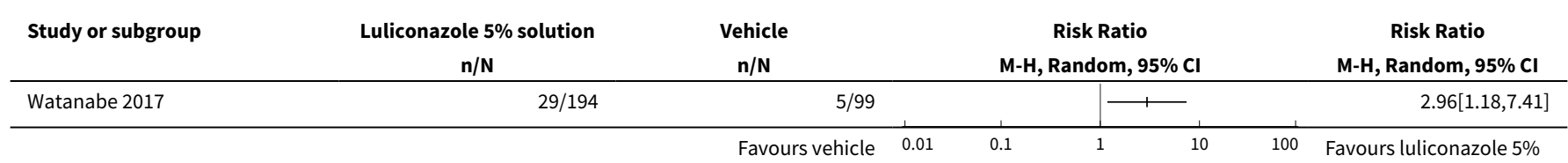

Analysis 6.2. Comparison 6 Luliconazole 5\% solution vs vehicle, Outcome 2 Adverse events.

\begin{tabular}{|c|c|c|c|c|c|}
\hline Study or subgroup & $\begin{array}{l}\text { Luliconazole } 5 \% \text { solution } \\
\qquad \mathrm{n} / \mathrm{N}\end{array}$ & $\begin{array}{c}\text { Vehicle } \\
\mathrm{n} / \mathrm{N}\end{array}$ & & $\begin{array}{c}\text { Risk Ratio } \\
\text { M-H, Random, } 95 \% \mathrm{Cl}\end{array}$ & $\begin{array}{c}\text { Risk Ratio } \\
\text { M-H, Random, } 95 \% \mathrm{CI}\end{array}$ \\
\hline Watanabe 2017 & $152 / 194$ & $76 / 99$ & & + & $1.02[0.9,1.16]$ \\
\hline
\end{tabular}

Analysis 6.3. Comparison 6 Luliconazole 5\% solution vs vehicle, Outcome 3 Mycological cure: 48 weeks.

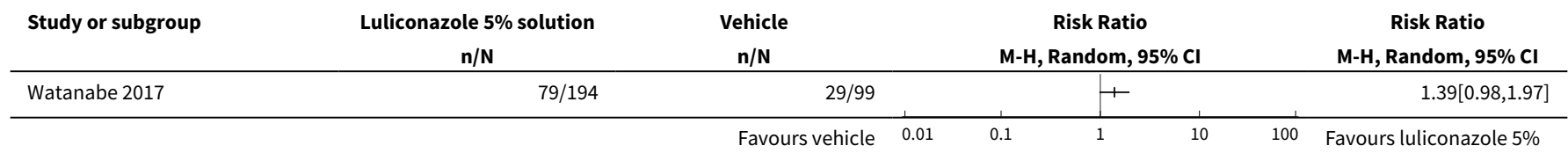

Comparison 7. Spirulina extract vs placebo

\begin{tabular}{lllll}
\hline Outcome or subgroup title & $\begin{array}{l}\text { No. of } \\
\text { studies }\end{array}$ & $\begin{array}{l}\text { No. of par- } \\
\text { ticipants }\end{array}$ & Statistical method & Effect size \\
\hline 1 Mycological cure: $12-36$ weeks & 1 & Risk Ratio (M-H, Random, 95\% Cl) & Totals not selected \\
\hline
\end{tabular}


Analysis 7.1. Comparison 7 Spirulina extract vs placebo, Outcome 1 Mycological cure: 12 - 36 weeks.

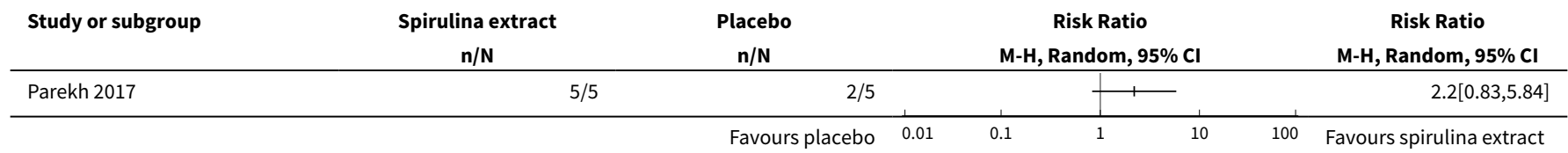

Comparison 8. Resin lacquer $30 \%$ vs amorolfine $5 \%$ lacquer

\begin{tabular}{lllll}
\hline Outcome or subgroup title & $\begin{array}{l}\text { No. of } \\
\text { studies }\end{array}$ & $\begin{array}{l}\text { No. of } \\
\text { partici- } \\
\text { pants }\end{array}$ & Statistical method & Effect size \\
\hline $\begin{array}{l}1 \text { Mycological cure: } 12-36 \\
\text { weeks }\end{array}$ & 1 & Risk Ratio (M-H, Random, $95 \% \mathrm{Cl})$ & Totals not selected \\
\hline $\begin{array}{l}2 \text { Mycological cure: } 36-52 \\
\text { weeks }\end{array}$ & 1 & Risk Ratio (M-H, Random, $95 \% \mathrm{Cl})$ & Totals not selected \\
\hline 3 Compliance (self-report) & 1 & Risk Ratio (M-H, Random, $95 \% \mathrm{Cl})$ & Totals not selected \\
\hline
\end{tabular}

Analysis 8.1. Comparison 8 Resin lacquer $30 \%$ vs amorolfine 5\% lacquer, Outcome 1 Mycological cure: 12 - 36 weeks.

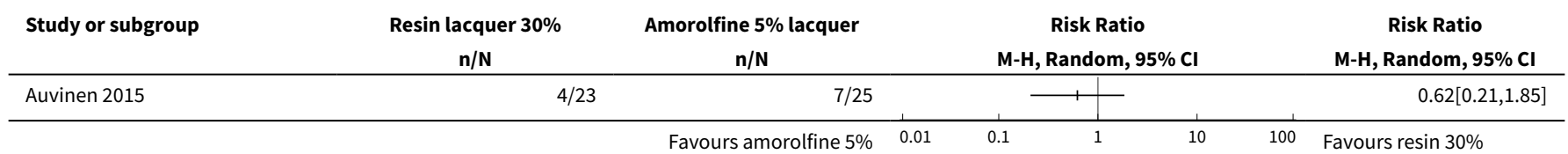

Analysis 8.2. Comparison 8 Resin lacquer 30\% vs amorolfine 5\% lacquer, Outcome 2 Mycological cure: 36 - 52 weeks.

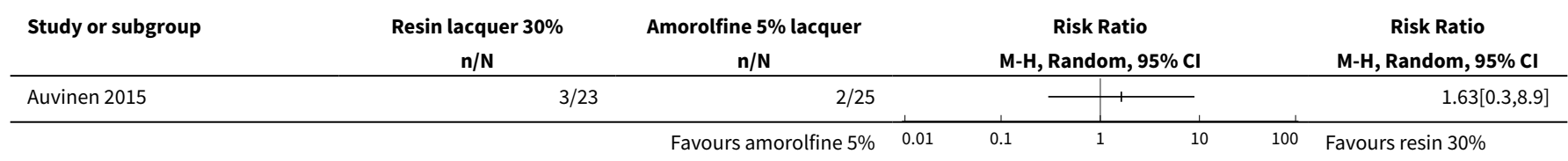

Analysis 8.3. Comparison 8 Resin lacquer $30 \%$ vs amorolfine $5 \%$ lacquer, Outcome 3 Compliance (self-report).

\begin{tabular}{|c|c|c|c|c|}
\hline Study or subgroup & $\begin{array}{c}\text { Resin lacquer } 30 \% \\
\mathbf{n} / \mathbf{N}\end{array}$ & $\begin{array}{c}\text { Amorolfine } 5 \% \text { lacquer } \\
\mathrm{n} / \mathrm{N}\end{array}$ & $\begin{array}{c}\text { Risk Ratio } \\
\text { M-H, Random, 95\% Cl }\end{array}$ & $\begin{array}{c}\text { Risk Ratio } \\
\text { M-H, Random, } 95 \% \mathrm{CI}\end{array}$ \\
\hline Auvinen 2015 & $23 / 23$ & $24 / 25$ & \begin{tabular}{l|l}
1 \\
\end{tabular} & $1.04[0.93,1.16]$ \\
\hline
\end{tabular}


Comparison 9. Amorolfine $5 \%$ nail lacquer: nail varnish vs no nail varnish

\begin{tabular}{lllll}
\hline Outcome or subgroup title & $\begin{array}{l}\text { No. of } \\
\text { studies }\end{array}$ & $\begin{array}{l}\text { No. of } \\
\text { partici- } \\
\text { pants }\end{array}$ & Statistical method & Effect size \\
\hline 1 Adverse events & 1 & Risk Ratio (M-H, Random, 95\% Cl) & Totals not selected \\
\hline 2 Mycological cure: 12 weeks & 1 & Risk Ratio (M-H, Random, 95\% Cl) & Totals not selected \\
\hline
\end{tabular}

Analysis 9.1. Comparison 9 Amorolfine $5 \%$ nail lacquer: nail varnish vs no nail varnish, Outcome 1 Adverse events.

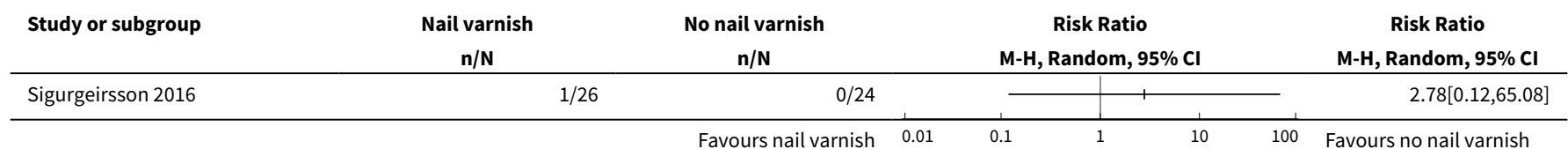

Analysis 9.2. Comparison 9 Amorolfine $5 \%$ nail lacquer: nail varnish vs no nail varnish, Outcome 2 Mycological cure: 12 weeks.

\begin{tabular}{|c|c|c|c|c|}
\hline Study or subgroup & $\begin{array}{c}\text { Nail varnish } \\
\mathrm{n} / \mathrm{N}\end{array}$ & $\begin{array}{c}\text { No nail varnish } \\
\mathrm{n} / \mathrm{N} \\
\end{array}$ & $\begin{array}{c}\text { Risk Ratio } \\
\text { M-H, Random, 95\% CI }\end{array}$ & $\begin{array}{c}\text { Risk Ratio } \\
\text { M-H, Random, } 95 \% \mathrm{Cl} \\
\end{array}$ \\
\hline Sigurgeirsson 2016 & $25 / 26$ & $23 / 24$ & 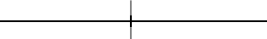 & $1[0.9,1.12]$ \\
\hline
\end{tabular}

Comparison 10. Acetic acid-based peelable nail polish vs amorolfine $\mathbf{5 \%}$ lacquer

\begin{tabular}{lllll}
\hline Outcome or subgroup title & $\begin{array}{l}\text { No. of } \\
\text { studies }\end{array}$ & $\begin{array}{l}\text { No. of par- } \\
\text { ticipants }\end{array}$ & Statistical method & Effect size \\
\hline 1 Mycological cure: $12-36$ weeks & 1 & Risk Ratio (M-H, Random, 95\% Cl) & Totals not selected \\
\hline
\end{tabular}

Analysis 10.1. Comparison 10 Acetic acid-based peelable nail polish vs amorolfine 5\% lacquer, Outcome 1 Mycological cure: 12 - 36 weeks.

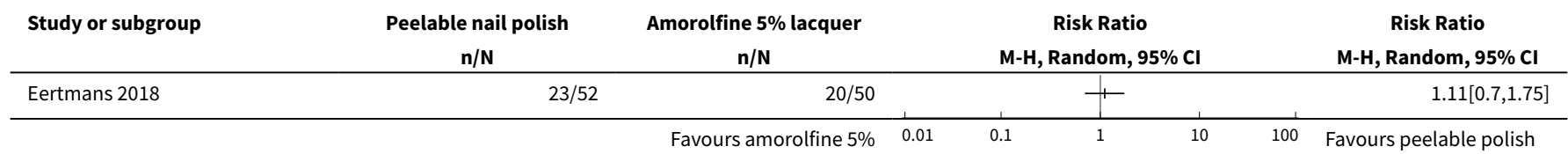


Comparison 11. Photodynamic therapy vs intense pulsed light therapy

\begin{tabular}{lllll}
\hline Outcome or subgroup title & $\begin{array}{l}\text { No. of } \\
\text { studies }\end{array}$ & $\begin{array}{l}\text { No. of } \\
\text { partici- } \\
\text { pants }\end{array}$ & Statistical method & Effect size \\
\hline 1 Complete cure: $12-36$ weeks & 1 & Risk Ratio (M-H, Random, $95 \% \mathrm{Cl})$ & Totals not selected \\
\hline 2 Mycological cure: $12-36$ weeks & 1 & Risk Ratio (M-H, Random, $95 \% \mathrm{Cl})$ & Totals not selected \\
\hline
\end{tabular}

Analysis 11.1. Comparison 11 Photodynamic therapy vs intense pulsed light therapy, Outcome 1 Complete cure: 12 - 36 weeks.

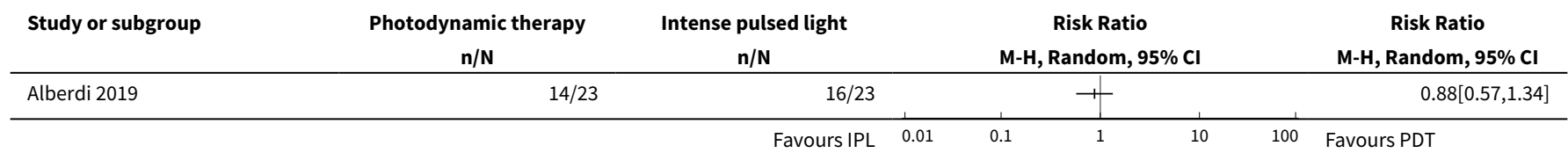

Analysis 11.2. Comparison 11 Photodynamic therapy vs intense pulsed light therapy, Outcome 2 Mycological cure: 12 - 36 weeks.

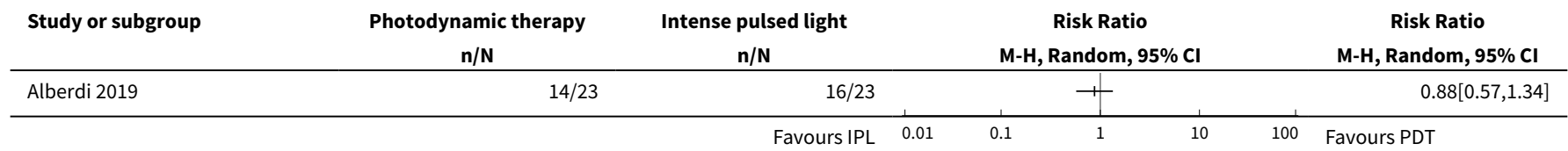

Comparison 12. Fractional laser and photodynamic therapy vs fractional laser and amorolfine 5\% lacquer

\begin{tabular}{lllll}
\hline Outcome or subgroup title & $\begin{array}{l}\text { No. of } \\
\text { studies }\end{array}$ & $\begin{array}{l}\text { No. of } \\
\text { partici- } \\
\text { pants }\end{array}$ & Statistical method & Effect size \\
\hline 1 Mycological cure: 12 weeks & 1 & & Risk Ratio (M-H, Random, $95 \% \mathrm{Cl})$ & Totals not selected \\
\hline 2 Mycological cure: $36-52$ weeks & 1 & Risk Ratio (M-H, Random, $95 \% \mathrm{Cl})$ & Totals not selected \\
\hline
\end{tabular}

Analysis 12.1. Comparison 12 Fractional laser and photodynamic therapy vs fractional laser and amorolfine 5\% lacquer, Outcome 1 Mycological cure: 12 weeks.

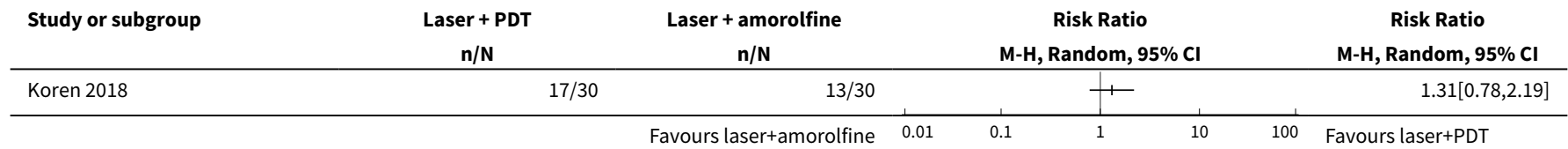


Analysis 12.2. Comparison 12 Fractional laser and photodynamic therapy vs fractional laser and amorolfine 5\% lacquer, Outcome 2 Mycological cure: 36 - 52 weeks.

\begin{tabular}{|c|c|c|c|c|c|c|c|}
\hline Study or subgroup & $\begin{array}{c}\text { Laser + PDT } \\
n / \mathbf{N}\end{array}$ & $\begin{array}{c}\text { Laser + amorolfine } \\
\mathrm{n} / \mathrm{N}\end{array}$ & & & $\begin{array}{l}\text { k Ratio } \\
\text { dom, } 95 \% \mathrm{Cl}\end{array}$ & & $\begin{array}{c}\text { Risk Ratio } \\
\text { M-H, Random, } 95 \% \mathrm{CI}\end{array}$ \\
\hline Koren 2018 & $8 / 30$ & $4 / 30$ & & & \begin{tabular}{l|l}
1 \\
\end{tabular} & & $2[0.67,5.94]$ \\
\hline
\end{tabular}

\section{ADDITIONAL TABLES}

Table 1. Glossary of terms

\begin{tabular}{|c|c|}
\hline Term & Definition \\
\hline Allylamine & $\begin{array}{l}\text { Class of antifungal molecules used to treat skin and nails. Examples include butenafine and } \\
\text { terbinafine }\end{array}$ \\
\hline Azole & $\begin{array}{l}\text { Class of antifungal compounds used to treat skin and nails, characterised by a 5-membered nitro- } \\
\text { gen heterocyclic ring. Examples include ketoconazole and fluconazole }\end{array}$ \\
\hline Benzoxaborole & $\begin{array}{l}\text { Class of small molecules characterised by a boron atom. The antifungal tavaborole is a member of } \\
\text { this class }\end{array}$ \\
\hline Cross-contamination & Unintentional transfer of a micro-organism from 1 object or person to another \\
\hline Dermatophytes & $\begin{array}{l}\text { Infectious fungi that feed on the keratin present in the skin, hair, and nails, which usually cause an } \\
\text { inflammatory response at the site of infection }\end{array}$ \\
\hline Distal & Away from the cuticle, toward the end of the nail \\
\hline Dystrophic & Progressive damage or deformity characterised by the wasting away of tissue or both \\
\hline Fungicidal & To kill fungal cells \\
\hline Fungistatic & To impede the growth and reproduction of fungal cells \\
\hline Hydroxypyridone & Class of compounds with antifungal properties used to treat skin and nails. Example: ciclopirox \\
\hline Hyponychium & The skin beneath the distal free edge of the nail \\
\hline lontophoresis & $\begin{array}{l}\text { A method whereby ionic medicinal compounds are introduced into the body by applying an electri- } \\
\text { cal current to the skin }\end{array}$ \\
\hline Keratin & A key protein in the structure of skin, hair, and nails \\
\hline Lateral & To the sides (of the nail) \\
\hline PCR & $\begin{array}{l}\text { Polymerase chain reaction, a molecular biology technique that uses DNA amplification to identify } \\
\text { organisms, such as fungi }\end{array}$ \\
\hline Proximal & Close to (the cuticle) \\
\hline Metalloproteases & Enzymes in the body that break down proteins and contain a metal ion to aid in this process \\
\hline Morpholine derivative & An antifungal based on the organic compound morpholine. Example: amorolfine \\
\hline
\end{tabular}


Table 1. Glossary of terms (Continued)

\begin{tabular}{ll} 
Mycological & Concerning fungi \\
\hline Nail apparatus & Refers to the nail and associated underlying structures (Figure 1) \\
\hline Onycholysis & Detachment of the nail from the nail bed \\
\hline Subungual & Underneath the nail \\
\hline Synthetase & A biological enzyme that acts in the body to combine molecules or compounds together \\
\hline Tinea pedis & Also known as 'athlete's foot', an infection of skin of the feet typically caused by dermatophytes
\end{tabular}

Table 2. Correspondence with study authors

\begin{tabular}{|c|c|c|c|}
\hline Study & Contact \& Date & Information Requested & Response \\
\hline \multicolumn{4}{|l|}{$\begin{array}{l}\text { Included } \\
\text { studies }\end{array}$} \\
\hline $\begin{array}{l}\text { AC- } \\
\text { TRN12614000 }\end{array}$ & $\begin{array}{l}\text { Dr. J. Thomas } \\
946617 \\
\text { Email correspondence } \\
\text { July } 2017\end{array}$ & Update on study & $\begin{array}{l}\text { Email response received July } 2017 . \\
\text { Manuscript is currently under review. }\end{array}$ \\
\hline $\begin{array}{l}\text { AC- } \\
\text { TRN126140012 }\end{array}$ & $\begin{array}{l}\text { Dr. T. Altaei } \\
238662 \\
\text { Email correspondence } \\
\text { July } 2017 \\
\text { June } 2019\end{array}$ & Update on study & $\begin{array}{l}\text { Email response received July } 2017 . \\
\text { No further response. } \\
\text { Manuscript is currently in preparation } \\
\text { for publication. }\end{array}$ \\
\hline Alberdi 2019 & $\begin{array}{l}\text { Dr. C. Gomez } \\
\text { Email correspondence } \\
\text { June } 2019\end{array}$ & $\begin{array}{l}\text { Allocation concealment and blind- } \\
\text { ing procedures; was the study regis- } \\
\text { tered? }\end{array}$ & $\begin{array}{l}\text { Email response received June } 2019 . \\
\text { Responses recorded in Characteris- } \\
\text { tics of included studies table. }\end{array}$ \\
\hline $\begin{array}{l}\text { Amichai } \\
2010\end{array}$ & $\begin{array}{l}\text { Dr. B. Amichai } \\
\text { Email correspondence } \\
\text { May } 2017\end{array}$ & $\begin{array}{l}\text { Randomisation and blinding proce- } \\
\text { dures; } \\
\text { treatment received by protocol viola- } \\
\text { tions and those experiencing AEs }\end{array}$ & No response \\
\hline Baran 2009 & $\begin{array}{l}\text { Dr. R. Baran } \\
\text { Email correspondence } \\
\text { August } 2017\end{array}$ & $\begin{array}{l}\text { Blinding procedures; reasons for at- } \\
\text { trition }\end{array}$ & No response \\
\hline $\begin{array}{l}\text { Bonhert } \\
2019\end{array}$ & $\begin{array}{l}\text { Dr. N. Sadick } \\
\text { Email correspondence } \\
\text { June } 2019\end{array}$ & $\begin{array}{l}\text { Randomisation, allocation conceal- } \\
\text { ment, blinding procedures; any infor- } \\
\text { mation on adverse events for efina- } \\
\text { conazole; was the trial registered? }\end{array}$ & No response \\
\hline Buck 1994 & Dr. D. Buck & Treatment allocation of drop-outs & No response \\
\hline
\end{tabular}


Table 2. Correspondence with study authors (Continued)

Research Gate

August 2017

\begin{tabular}{|c|c|c|c|}
\hline $\begin{array}{l}\text { Eertmans } \\
2018\end{array}$ & $\begin{array}{l}\text { Dr. F. Eertmans } \\
\text { Email correspondence }\end{array}$ & $\begin{array}{l}\text { Allocation concealment, outcome as- } \\
\text { sessor blinding }\end{array}$ & $\begin{array}{l}\text { Email response received June } 2019 . \\
\text { Responses recorded in Characteris- } \\
\text { tics of included studies table }\end{array}$ \\
\hline & June 2019 & & \\
\hline
\end{tabular}

\begin{tabular}{|c|c|c|c|}
\hline $\begin{array}{l}\text { Elews- } \\
\text { ki 2013c; } \\
\text { Elewski } \\
\text { 2013d }\end{array}$ & $\begin{array}{l}\text { Mr. B. Bulley } \\
\text { Email correspondence } \\
\text { August } 2017\end{array}$ & $\begin{array}{l}\text { What do the percentages of AEs not } \\
\text { related to study drug in the article re- } \\
\text { fer to? }\end{array}$ & $\begin{array}{l}\text { Email response received August 2017: } \\
\text { total number of AEs reported, not } \\
\text { number of patients. For study 1, 1047 } \\
\text { and } 352 \text { (efinaconazole and vehicle) } \\
\text { and study } 2,908 \text { and } 278 \text { (efinacona- } \\
\text { zole and vehicle) }\end{array}$ \\
\hline
\end{tabular}

\begin{tabular}{|c|c|c|c|}
\hline $\begin{array}{l}\text { Elews- } \\
\text { ki 2015a; } \\
\text { Elewski } \\
2015 b\end{array}$ & $\begin{array}{l}\text { Dr. N. Lowe, Sandoz } \\
\text { Email correspondence }\end{array}$ & $\begin{array}{l}\text { Randomisation and blinding proce- } \\
\text { dures }\end{array}$ & $\begin{array}{l}\text { Email response received May } 2017 . \\
\text { Responses recorded in Characteris- } \\
\text { tics of included studies table }\end{array}$ \\
\hline
\end{tabular}

May 2017

\begin{tabular}{ccll}
\hline $\begin{array}{l}\text { EUC- } \\
\text { TR2005-005905-51 } \\
\text { Email correspondence } \\
\text { September 2018 }\end{array}$ & $\begin{array}{l}\text { Randomisation and blinding proce- } \\
\text { dures; are results available?; reasons } \\
\text { for attrition }\end{array}$ & $\begin{array}{l}\text { Email response received Sept 2018. } \\
\text { Inquiry being directed to appropriate } \\
\text { person }\end{array}$ \\
\hline $\begin{array}{l}\text { EUC- } \\
\text { TR2006-000974-54 Sidou, Galderma } \\
\text { Email correspondence }\end{array}$ & $\begin{array}{l}\text { Randomisation and blinding proce- } \\
\text { dures; are results available?; possible } \\
\text { conflicts of interest for study investi- } \\
\text { gators }\end{array}$ & $\begin{array}{l}\text { Email response received August 2017: } \\
\text { tion on person who conducted the } \\
\text { study, will inform }\end{array}$ \\
& & - No further correspondence \\
\hline
\end{tabular}

\begin{tabular}{ccl}
\hline $\begin{array}{c}\text { EUC- } \\
\text { TR2006-005895-42 } \\
\text { Research Gate } \\
\text { September 2018 }\end{array}$ & $\begin{array}{l}\text { Randomisation and blinding proce- } \\
\text { dures; are results available?; reasons } \\
\text { for attrition }\end{array}$ & No response \\
\end{tabular}

\begin{tabular}{|c|c|c|c|}
\hline $\begin{array}{l}\text { EUC- } \\
\text { TR2008-00321 }\end{array}$ & $\begin{array}{l}\text { Bayer website contact form } \\
\text { 5-13 } \\
\text { August } 2017 \\
\text { Consumer relations } \\
\text { Email correspondence } \\
\text { September } 2017\end{array}$ & $\begin{array}{l}\text { Randomisation and blinding proce- } \\
\text { dures; are results available? reasons } \\
\text { for attrition; possible conflicts of in- } \\
\text { terest for study investigators }\end{array}$ & No response \\
\hline $\begin{array}{l}\text { EUC- } \\
\text { TR2016-00124 }\end{array}$ & $\begin{array}{l}\text { Dr. J. Dobmeyer, Blueberry Thera- } \\
2 \text { peutics } \\
\text { Email correspondence } \\
\text { June } 2019\end{array}$ & $\begin{array}{l}\text { Randomisation, allocation conceal- } \\
\text { ment, blinding procedures; any up- } \\
\text { date on study results? }\end{array}$ & $\begin{array}{l}\text { Email response received July } 2019 . \\
\text { Responses recorded in Characteris- } \\
\text { tics of included studies table }\end{array}$ \\
\hline $\begin{array}{l}\text { Gupta } \\
2000 a \text {; Gup- } \\
\text { ta } 2000 \text { b }\end{array}$ & Dr. A.K. Gupta & $\begin{array}{l}\text { Randomisation and blinding proce- } \\
\text { dures }\end{array}$ & No further information available \\
\hline
\end{tabular}


Table 2. Correspondence with study authors (Continued)
Gupta 2006 Dr. A.K. Gupta
Randomisation and blinding proce-
No further information available dures; attrition data

\begin{tabular}{llll}
\hline $\begin{array}{l}\text { Hartmane } \\
\mathbf{2 0 1 3}\end{array}$ & Dr. M. Caserini, Polichem & $\begin{array}{l}\text { Randomisation and blinding proce- } \\
\text { dures; availability of results, poster or } \\
\text { published; reasons for attrition }\end{array}$ & $\begin{array}{l}\text { Email response received Aug. 2017 } \\
\text { Email correspondence }\end{array}$ \\
\hline $\begin{array}{l}\text { August 2017 } \\
\mathbf{2 0 1 4}\end{array}$ & Dr. J.Y. Tang & Allocation concealment procedure & Email response received Sept. 2018 \\
& Email correspondence & & $\begin{array}{l}\text { Responses recorded in } \text { Characteris- } \\
\text { tics of included studies table. }\end{array}$ \\
\hline
\end{tabular}

\begin{tabular}{ll}
\hline ljzerman & Dr. M. Ijzerman, Millendo \\
2010 & Ms. L. Scallion, NanoBio \\
& Email correspondence
\end{tabular}

May 2017

Is there more information about this study? Has it been published or are there e-posters?
Email response received May 2017

No longer at company. Provided L. Scallion's contact information.

- No further response

\begin{tabular}{|c|c|c|c|}
\hline Iorizzo 2016 & $\begin{array}{l}\text { Dr. M. Iorizzo } \\
\text { Email correspondence } \\
\text { September } 2017\end{array}$ & $\begin{array}{l}\text { Randomisation procedures; any attri- } \\
\text { tion data }\end{array}$ & $\begin{array}{l}\text { Email response received Sept. } 2017 \\
\text { Responses recorded in Characteris- } \\
\text { tics of included studies table }\end{array}$ \\
\hline Koren 2018 & $\begin{array}{l}\text { Dr. A. Koren } \\
\text { Email correspondence } \\
\text { June } 2019\end{array}$ & $\begin{array}{l}\text { Randomisation, allocation conceal- } \\
\text { ment, blinding procedures; was the } \\
\text { study registered? }\end{array}$ & $\begin{array}{l}\text { Email response received June } 2019 \\
\text { Responses recorded in Characteris- } \\
\text { tics of included studies table }\end{array}$ \\
\hline Kumar 2001 & $\begin{array}{l}\text { Dr. K. Kulkarni } \\
\text { Email correspondence } \\
\text { September } 2017\end{array}$ & $\begin{array}{l}\text { Randomisation and blinding proce- } \\
\text { dures; trial registration }\end{array}$ & No response \\
\hline
\end{tabular}

\begin{tabular}{|c|c|c|c|}
\hline Lahfa 2013 & $\begin{array}{l}\text { Dr. M. Lahfa } \\
\text { Email correspondence } \\
\text { August } 2017\end{array}$ & Reasons for participant withdrawal & No response \\
\hline Lu 2005 & $\begin{array}{l}\text { Dr. Lu } \\
\text { Email correspondence } \\
\text { May } 2017 \\
\text { Apricus Biosciences } \\
\text { Web contact form } \\
\text { September } 2018\end{array}$ & $\begin{array}{l}\text { Randomisation and blinding proce- } \\
\text { dures; are results available? }\end{array}$ & $\begin{array}{l}\text { Automatic email response: no longer } \\
\text { with company } \\
\text { - No further response from web form }\end{array}$ \\
\hline $\begin{array}{l}\text { Montana } \\
1994\end{array}$ & $\begin{array}{l}\text { Dr. R.K. Scher } \\
\text { Email correspondence } \\
\text { August } 2017\end{array}$ & $\begin{array}{l}\text { Randomisation and blinding proce- } \\
\text { dures; attrition data }\end{array}$ & $\begin{array}{l}\text { Email response received August } 2017 \\
\text { Unable to provide information. Re- } \\
\text { ferred to Columbia Dermatology. }\end{array}$ \\
\hline
\end{tabular}


Table 2. Correspondence with study authors (Continued)

Columbia Dermatology

Email correspondence

September 2018

\begin{tabular}{|c|c|c|c|}
\hline NCT01080079 & $\begin{array}{l}\text { NitrcBio Therapeutics } \\
\text { Email Correspondence } \\
\text { September } 2017\end{array}$ & Are results available for the trial? & No response \\
\hline NCT01145807 & $\begin{array}{l}\text { Dr. E. Tschen } \\
\text { Email Correspondence } \\
\text { September } 2017\end{array}$ & Are results available for the trial? & No response \\
\hline NCT01246518 & $\begin{array}{l}\text { Dr. K. Rensfeldt, Moberg Pharma } \\
\text { Email Correspondence } \\
\text { September } 2018\end{array}$ & $\begin{array}{l}\text { Randomisation and allocation con- } \\
\text { cealment procedures; are results } \\
\text { available or published? }\end{array}$ & $\begin{array}{l}\text { Email response received Oct } 2018 . \\
\text { Data is not available. Trial procedures } \\
\text { documented in Characteristics of in- } \\
\text { cluded studies table. }\end{array}$ \\
\hline NCT01400594 & $\begin{array}{l}\text { Hisamitsu Pharmaceutical } \\
\text { Web contact form } \\
\text { September } 2018\end{array}$ & $\begin{array}{l}\text { Randomisation, allocation conceal- } \\
\text { ment, blinding procedures; attrition } \\
\text { data; are results available or pub- } \\
\text { lished? }\end{array}$ & No response \\
\hline NCT02022215 & $\begin{array}{l}\text { Meiji Seika Pharma } \\
\text { Email Correspondence } \\
\text { September } 2017\end{array}$ & Are results available for the trial? & No response \\
\hline NCT02343627 & $\begin{array}{l}\text { Taro Pharmaceuticals USA } \\
\text { Email Correspondence } \\
\text { September } 2017\end{array}$ & Are results available for the trial? & $\begin{array}{l}\text { No response, results posted on clini- } \\
\text { caltrials.gov } 22 \text { August } 2018\end{array}$ \\
\hline NCT03141840 & $\begin{array}{l}\text { Dr. I. Mouratidou-Kontorinis } \\
\text { Email Correspondence } \\
\text { June } 2019\end{array}$ & $\begin{array}{l}\text { Randomisation, allocation conceal- } \\
\text { ment, blinding procedures; attrition } \\
\text { data; are results available or pub- } \\
\text { lished? }\end{array}$ & $\begin{array}{l}\text { Email response received June } 2019 . \\
\text { Data is not yet available. Trial proce- } \\
\text { dures documented in Characteristics } \\
\text { of included studies table }\end{array}$ \\
\hline Parekh 2017 & $\begin{array}{l}\text { Leodevico L. Ilag } \\
\text { Email correspondence } \\
\text { April } 2019\end{array}$ & $\begin{array}{l}\text { Were fingernails and toenails treat- } \\
\text { ed? }\end{array}$ & $\begin{array}{l}\text { Email response received April } 2019 . \\
\text { Confirmed that toenails were includ- } \\
\text { ed in study and that fingernails were } \\
\text { not }\end{array}$ \\
\hline Paul 2013 & $\begin{array}{l}\text { Dr. C. Paul } \\
\text { Email correspondence } \\
\text { August } 2017\end{array}$ & Randomisation procedures & $\begin{array}{l}\text { Email forwarded to Pierre Fabre. Re- } \\
\text { sponse received September } 2017 . \\
\text { Responses recorded in Characteris- } \\
\text { tics of included studies table }\end{array}$ \\
\hline $\begin{array}{l}\text { Romero- } \\
\text { Cerecero } \\
2008\end{array}$ & $\begin{array}{l}\text { Dr. O. Romero-Cerecero } \\
\text { Email correspondence }\end{array}$ & $\begin{array}{l}\text { Randomisation and blinding proce- } \\
\text { dures; trial registration }\end{array}$ & No response \\
\hline
\end{tabular}

Topical and device-based treatments for fungal infections of the toenails (Review)

Copyright $\odot 2020$ The Cochrane Collaboration. Published by John Wiley \& Sons, Ltd. 
Table 2. Correspondence with study authors (Continued) August 2017

\begin{tabular}{|c|c|c|c|}
\hline $\begin{array}{l}\text { Romero- } \\
\text { Cerecero } \\
2009\end{array}$ & $\begin{array}{l}\text { Dr. O. Romero-Cerecero } \\
\text { Dr. J. Tortoriello } \\
\text { Email correspondence } \\
\text { August } 2017\end{array}$ & $\begin{array}{l}\text { Randomisation and blinding proce- } \\
\text { dures; trial registration; treatment } \\
\text { regimen }\end{array}$ & No response \\
\hline $\begin{array}{l}\text { Schalka } \\
2012\end{array}$ & $\begin{array}{l}\text { Dr. S. Schalka } \\
\text { Email correspondence } \\
\text { August } 2017\end{array}$ & $\begin{array}{l}\text { Randomisation and blinding proce- } \\
\text { dures; trial registration }\end{array}$ & No response \\
\hline Stein 2014 & $\begin{array}{l}\text { Dr. L. Stein Gold } \\
\text { Email correspondence } \\
\text { May } 2017\end{array}$ & Are results available or published? & $\begin{array}{l}\text { Email response received May } 2017 . \\
\text { No additional information available }\end{array}$ \\
\hline $\begin{array}{l}\text { Syed 1998; } \\
\text { Syed } 1999\end{array}$ & $\begin{array}{l}\text { Dr. T.A. Syed } \\
\text { Research Gate } \\
\text { August } 2017\end{array}$ & Randomisation procedures & No response \\
\hline $\begin{array}{l}\text { Toledo-Ba- } \\
\text { hena } 2014\end{array}$ & $\begin{array}{l}\text { Dr. N. Lowe, Sandoz } \\
\text { Email correspondence } \\
\text { May } 2017\end{array}$ & $\begin{array}{l}\text { Randomisation and blinding proce- } \\
\text { dures }\end{array}$ & $\begin{array}{l}\text { Email response received May } 2017 . \\
\text { Responses recorded in Characteris- } \\
\text { tics of included studies table. }\end{array}$ \\
\hline Tschen 2013 & $\begin{array}{l}\text { Mr. B. Bulley } \\
\text { Email correspondence } \\
\text { May } 2017\end{array}$ & $\begin{array}{l}\text { Blinding procedures; mycological } \\
\text { cure rate for control group }\end{array}$ & $\begin{array}{l}\text { Email response received May } 2017 . \\
\text { Responses recorded in Characteris- } \\
\text { tics of included studies table. }\end{array}$ \\
\hline Waibel 2013 & $\begin{array}{l}\text { Dr. J. Waibel } \\
\text { Email correspondence } \\
\text { August } 2017\end{array}$ & $\begin{array}{l}\text { Randomisation and blinding proce- } \\
\text { dures }\end{array}$ & No response \\
\hline $\begin{array}{l}\text { Watanabe } \\
2017\end{array}$ & $\begin{array}{l}\text { Dr. S. Watanabe } \\
\text { Email correspondence } \\
\text { August } 2017\end{array}$ & Blinding procedures & No response \\
\hline
\end{tabular}

\section{Excluded} studies

\begin{tabular}{llll}
\hline $\begin{array}{l}\text { Bassiri- } \\
\text { Jahromi } \\
2012\end{array}$ & Dr. S. Bassiri-Jahromi & Attrition data and outcome data & No response \\
& Email correspondence & & \\
& September 2017 & & \\
\hline $\begin{array}{l}\text { El-Tatawy } \\
2015\end{array}$ & Dr. R. El-Tatawy & Is data available for toenails alone? & No response \\
\hline
\end{tabular}


Table 2. Correspondence with study authors (Continued)

Email correspondence

September 2017

\begin{tabular}{|c|c|c|c|}
\hline $\begin{array}{l}\text { Emtestam } \\
2012\end{array}$ & $\begin{array}{l}\text { Dr. A. Toft, Moberg Pharma } \\
\text { Email correspondence } \\
\text { September } 2017\end{array}$ & $\begin{array}{l}\text { Are results/attrition data available for } \\
\text { toenails alone? }\end{array}$ & $\begin{array}{l}\text { Email response received September } \\
2017 \\
\text { Unable to find requested information }\end{array}$ \\
\hline $\begin{array}{l}\text { Gilaberte } \\
2017\end{array}$ & $\begin{array}{l}\text { Dr. Y. Gilaberte } \\
\text { Email correspondence } \\
\text { September } 2017\end{array}$ & Is data available for toenails alone? & $\begin{array}{l}\text { Email response received October } \\
2017 \\
\text { A separate analysis was not per- } \\
\text { formed }\end{array}$ \\
\hline Halmy 2005 & $\begin{array}{l}\text { Dr. K. Halmy } \\
\text { Email correspondence } \\
\text { August } 2017\end{array}$ & $\begin{array}{l}\text { Are data available? Has the study } \\
\text { been published? }\end{array}$ & No response \\
\hline
\end{tabular}

\begin{tabular}{ll}
\hline Kim 2016b Dr. S.J. Lee & Is data available for toenails alone? No response \\
& Email correspondence \\
& September 2017 \\
\hline
\end{tabular}

\begin{tabular}{lll}
\hline $\begin{array}{l}\text { Lauharanta } \\
1992\end{array}$ & $\begin{array}{l}\text { Dr. J. Lauharanta } \\
\text { Research Gate } \\
\text { September } 2017\end{array}$ & $\begin{array}{l}\text { Definitions of efficacy outcomes, data } \\
\text { available for toenails alone? }\end{array}$ \\
\end{tabular}

\begin{tabular}{ll}
\hline Li $2014 \quad$ Dr. Y. Li & Is data available for toenails alone? No response \\
& Email correspondence \\
& September 2017
\end{tabular}

\begin{tabular}{|c|c|c|c|}
\hline $\begin{array}{l}\text { Menéndez } \\
2011\end{array}$ & $\begin{array}{l}\text { Dr. S. Menendez } \\
\text { Email correspondence } \\
\text { May } 2017\end{array}$ & $\begin{array}{l}\text { Did this study include both fingernails } \\
\text { and toenails? If yes, are data avail- } \\
\text { able for toenails alone? }\end{array}$ & $\begin{array}{l}\text { Email response received May } 2017 \\
\text { Yes, the study included both finger- } \\
\text { nails and toenails. Original data not } \\
\text { accessible }\end{array}$ \\
\hline Tietz 2013 & $\begin{array}{l}\text { Dr. H. Merk } \\
\text { Email correspondence } \\
\text { September } 2017\end{array}$ & Is data available for toenails alone? & No response \\
\hline $\begin{array}{l}\text { Zhang } \\
2012 a\end{array}$ & $\begin{array}{l}\text { Dr. Zhang } \\
\text { Email correspondence } \\
\text { September } 2017\end{array}$ & Is data available for toenails alone? & No response \\
\hline Zhou 2016 & $\begin{array}{l}\text { Dr. B.R. Zhou } \\
\text { Email correspondence } \\
\text { September } 2017\end{array}$ & Is data available for toenails alone? & No response \\
\hline
\end{tabular}


Table 2. Correspondence with study authors (Continued)

\section{Ongoing \\ studies}

\begin{tabular}{|c|c|c|c|}
\hline \multicolumn{2}{|c|}{ DRKS00007709 Dr. Raulin } & Update on study & No response \\
\hline \multicolumn{4}{|c|}{ Email correspondence } \\
\hline \multicolumn{4}{|c|}{ September 2018} \\
\hline \multirow{3}{*}{$\begin{array}{l}\text { EUC- } \\
\text { TR2016-00120 }\end{array}$} & Dr. C. Strid, Moberg Pharma & Update on study & Email response received September \\
\hline & $\begin{array}{l}\text { 4-39 } \\
\text { Email correspondence }\end{array}$ & & 2018 \\
\hline & September 2018 & & $\begin{array}{l}\text { The study has not been completed } \\
\text { yet }\end{array}$ \\
\hline \multirow[t]{5}{*}{ NCT02961634 } & Dr. A.C. Prazias Massei & Update on study & Email undeliverable \\
\hline & Email correspondence & & No response \\
\hline & MIP Brasil Farma & & \\
\hline & Online Contact Form & & \\
\hline & September 2018 & & \\
\hline \multirow[t]{3}{*}{ NCT03814343 } & Dr. C. Leeyaphan & \multirow{3}{*}{$\begin{array}{l}\text { Is the study performed on toenail, fin- } \\
\text { gernails, or both? }\end{array}$} & Email response received May 2019 \\
\hline & Email correspondence & & Study is performed with toenails only \\
\hline & May 2019 & & \\
\hline
\end{tabular}

\section{Studies \\ awaiting \\ classifica- \\ tion}

\begin{tabular}{|c|c|c|c|}
\hline $\begin{array}{l}\text { Anonymous } \\
2002\end{array}$ & $\begin{array}{l}\text { Cochrane Skin Group (CSG) } \\
\text { Email correspondence } \\
\text { November } 2017\end{array}$ & Full-text article & Article not available in register \\
\hline $\begin{array}{l}\text { Barquero } \\
2007\end{array}$ & $\begin{array}{l}\text { Dr. N. Barquero } \\
\text { Research Gate } \\
\text { November } 2017\end{array}$ & $\begin{array}{l}\text { What are the ages of the patients? } \\
\text { Was this study randomised? }\end{array}$ & No response \\
\hline $\begin{array}{l}\text { Butareva } \\
1986\end{array}$ & $\begin{array}{l}\text { Cochrane Skin Group (CSG) } \\
\text { Email correspondence } \\
\text { November } 2017\end{array}$ & Full-text article & Article not available in register \\
\hline $\begin{array}{l}\text { Emokpare } \\
1977\end{array}$ & $\begin{array}{l}\text { Cochrane Skin Group (CSG) } \\
\text { Email correspondence } \\
\text { November } 2017\end{array}$ & Full-text article & Article not available in register \\
\hline $\begin{array}{l}\text { EUC- } \\
\text { TR2008-0024: }\end{array}$ & $\begin{array}{l}\text { Substipharm } \\
\text { 7-90 } \\
\text { Email correspondence }\end{array}$ & $\begin{array}{l}\text { Update on study data, how was ony- } \\
\text { chomycosis diagnosed? }\end{array}$ & No response \\
\hline
\end{tabular}

Topical and device-based treatments for fungal infections of the toenails (Review) 
Table 2. Correspondence with study authors (Continued)

September 2018

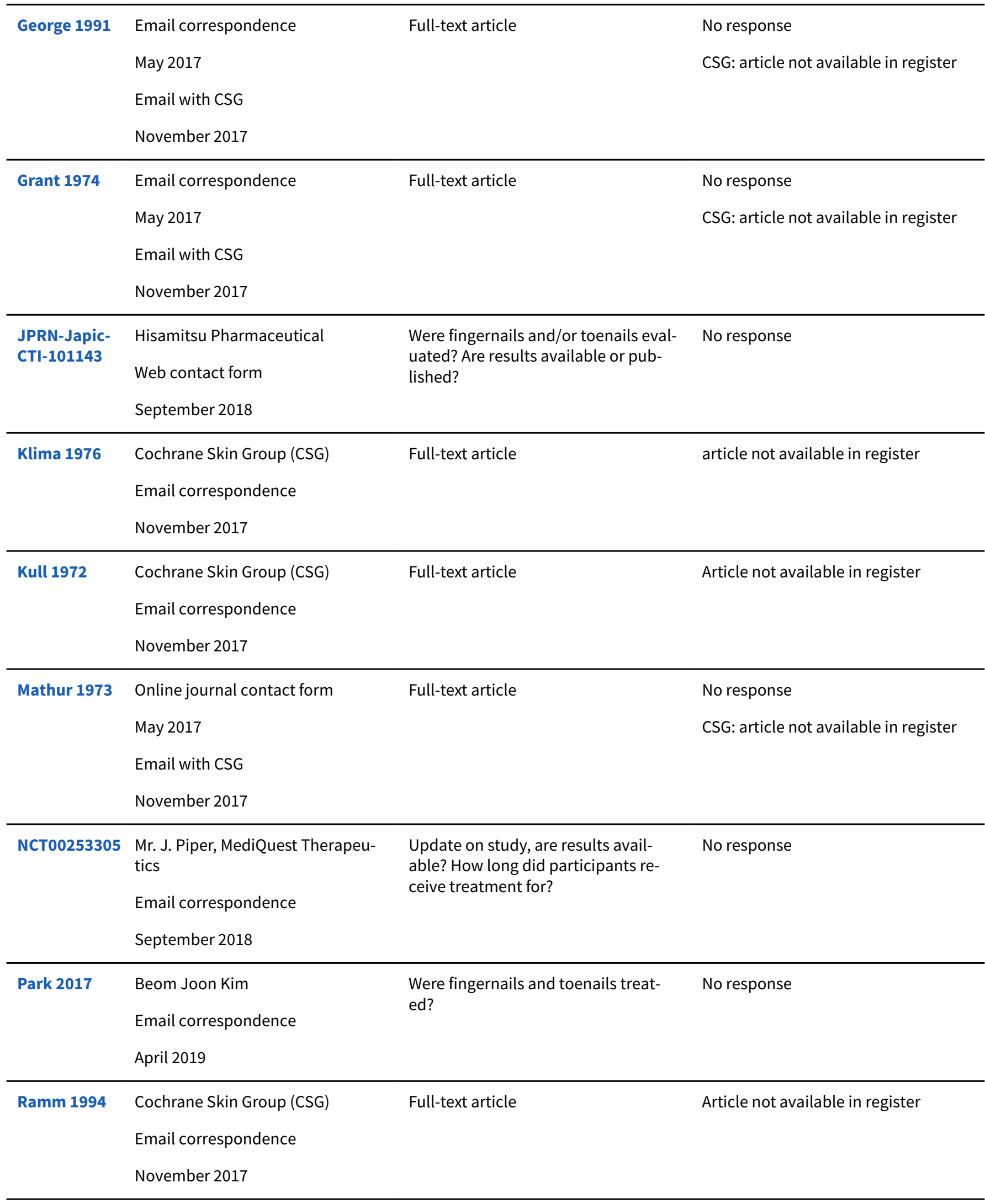


Table 2. Correspondence with study authors (Continued)

\begin{tabular}{|c|c|c|c|}
\hline $\begin{array}{l}\text { Rüping } \\
\text { 1993a }\end{array}$ & $\begin{array}{l}\text { Cochrane Skin Group (CSG) } \\
\text { Email correspondence } \\
\text { November } 2017\end{array}$ & Full-text article & Article not available in register \\
\hline $\begin{array}{l}\text { Rüping } \\
\text { 1993b }\end{array}$ & $\begin{array}{l}\text { Cochrane Skin Group (CSG) } \\
\text { Email correspondence } \\
\text { November } 2017\end{array}$ & Full-text article & Article not available in register \\
\hline $\begin{array}{l}\text { Schubert } \\
1973\end{array}$ & $\begin{array}{l}\text { Cochrane Skin Group (CSG) } \\
\text { Email correspondence } \\
\text { November } 2017\end{array}$ & Full-text article & Article not available in register \\
\hline Weuta 1972 & $\begin{array}{l}\text { Cochrane Skin Group (CSG) } \\
\text { Email correspondence } \\
\text { November } 2017\end{array}$ & Full-text article & Article not available in register \\
\hline Yao 2016 & $\begin{array}{l}\text { Dr. X.H. Wang } \\
\text { Email correspondence } \\
\text { May } 2017\end{array}$ & $\begin{array}{l}\text { Were fingernails and toenails treat- } \\
\text { ed? How were patients mycologically } \\
\text { diagnosed? }\end{array}$ & No response \\
\hline
\end{tabular}

Table 3. Summary of single studies

\begin{tabular}{|c|c|c|c|c|c|c|}
\hline & Treatments (n) & Treatment duration & $\begin{array}{l}\text { Complete } \\
\text { cure }\end{array}$ & $\begin{array}{l}\text { Adverse } \\
\text { events }\end{array}$ & $\begin{array}{l}\text { Mycological } \\
\text { cure }\end{array}$ & $\begin{array}{l}\text { Clinical } \\
\text { cure }\end{array}$ \\
\hline \multicolumn{7}{|c|}{ Topical treatment versus vehicle/placebo } \\
\hline \multirow[t]{5}{*}{$\begin{array}{l}\text { Baran } \\
2009 a\end{array}$} & $\begin{array}{l}\text { P-3051 (ciclopirox } 8 \% \text { hydro- } \\
\text { lacquer), } n=182\end{array}$ & $\begin{array}{l}\text { Once daily for } 48 \\
\text { weeks }\end{array}$ & $\begin{array}{l}10 / 182 \\
\text { RR } 10.90 \\
95 \% \mathrm{Cl} \\
0.65 \text { to } \\
184.03\end{array}$ & $\begin{array}{l}14 / 180 \\
\text { RR } 0.63,95 \% \\
\text { Cl } 0.30 \text { to } \\
1.31\end{array}$ & $\begin{array}{l}156 / 182 \\
\text { RR } 1.28,95 \% \mathrm{Cl} \\
1.10 \text { to } 1.49\end{array}$ & - \\
\hline & Placebo, $n=97$ & & $0 / 97$ & $12 / 97$ & $65 / 97$ & - \\
\hline & $\begin{array}{l}\text { Post hoc analysis: }<50 \% \text { nail } \\
\text { involvement at baseline mea- } \\
\text { sured at } 60 \text { weeks }\end{array}$ & - & - & - & - & - \\
\hline & $\begin{array}{l}\text { P-3051 (ciclopirox 8\% hydro- } \\
\text { lacquer), } n=119\end{array}$ & $\begin{array}{l}\text { Once daily for } 48 \\
\text { weeks }\end{array}$ & $\begin{array}{l}18 / 119 \\
\text { RR } 9.53, \\
95 \% \mathrm{Cl} \\
1.30 \text { to } \\
69.74\end{array}$ & - & $\begin{array}{l}98 / 119 \\
\text { RR } 1.08,95 \% \mathrm{Cl} \\
0.92 \text { to } 1.27\end{array}$ & - \\
\hline & Placebo, $n=63$ & & $1 / 63$ & - & $48 / 63$ & - \\
\hline
\end{tabular}


Table 3. Summary of single studies (Continued)

\begin{tabular}{|c|c|c|c|c|c|c|}
\hline \multirow[t]{4}{*}{$\begin{array}{l}\text { Elewski } \\
2013 a^{b}\end{array}$} & $\begin{array}{l}\text { Terbinafine nail solution (TNS), } \\
n=259\end{array}$ & \multirow[t]{2}{*}{$\begin{array}{l}\text { Once daily for } 24 \\
\text { weeks }\end{array}$} & $\begin{array}{l}3 / 259 \\
\text { RR } 1.49, \\
95 \% \mathrm{Cl} \\
0.25 \text { to } \\
8.87\end{array}$ & $\begin{array}{l}306 / 526 \\
\text { RR } 0.96,95 \% \\
\text { CI } 0.87 \text { to } \\
1.06\end{array}$ & $\begin{array}{l}33 / 259 \\
\text { RR } 2.05,95 \% \mathrm{Cl} \\
1.16 \text { to } 3.64\end{array}$ & $\begin{array}{l}6 / 259 \\
\text { RR } 1.00, \\
95 \% \mathrm{Cl} \\
0.33 \text { to } \\
3.05\end{array}$ \\
\hline & Vehicle, $\mathrm{n}=258$ & & $2 / 258$ & $308 / 507$ & $16 / 258$ & $6 / 258$ \\
\hline & TNS, $n=271$ & \multirow[t]{2}{*}{$\begin{array}{l}\text { Once daily for } 48 \\
\text { weeks }\end{array}$} & $\begin{array}{l}6 / 271 \\
\text { RR } 12.28, \\
95 \% \mathrm{Cl} \\
0.70 \text { to } \\
216.94\end{array}$ & $\begin{array}{l}176 / 269 \\
\text { RR } 0.91,95 \% \\
\mathrm{Cl} 0.81 \text { to } \\
1.02\end{array}$ & $\begin{array}{l}51 / 271 \\
\text { RR } 3.44,95 \% \mathrm{Cl} \\
1.95 \text { to } 6.06\end{array}$ & $\begin{array}{l}10 / 271 \\
\text { RR } 1.05, \\
95 \% \mathrm{Cl} \\
0.43 \text { to } \\
2.54\end{array}$ \\
\hline & Vehicle, $n=256$ & & $0 / 256$ & $183 / 254$ & $14 / 256$ & $9 / 256$ \\
\hline \multirow[t]{4}{*}{$\begin{array}{l}\text { EUC- } \\
\text { TR2006-0 }\end{array}$} & $\begin{array}{l}\text { Amorolfine 10\% lacquer, } n=31 \\
974-54\end{array}$ & \multirow{4}{*}{$\begin{array}{l}\text { Daily for } 2 \text { weeks, } \\
\text { then weekly until } 6 \\
\text { months }\end{array}$} & - & $7 / 31$ & - & - \\
\hline & Amorolfine $4 \%$ lacquer, $n=30$ & & - & $11 / 30$ & - & - \\
\hline & Vehicle lacquer, n = 34 & & - & $7 / 34$ & - & - \\
\hline & Amorolfine $5 \%$ lacquer, $n=32$ & & - & $12 / 32$ & - & - \\
\hline \multirow[t]{2}{*}{$\begin{array}{l}\text { EUC- } \\
\text { TR2008-0C }\end{array}$} & $\begin{array}{l}\text { Bifonazole } 1 \% \text { cream and urea } \\
2140 \% \% \text { paste, } n=347\end{array}$ & $\begin{array}{l}14-28 \text { days urea fol- } \\
\text { lowed by cream for } \\
28 \text { days }\end{array}$ & - & $\begin{array}{l}12 / 328 \\
\text { RR } 0.67,95 \% \\
\mathrm{Cl} 0.33 \text { to } \\
1.37\end{array}$ & $\begin{array}{l}156 / 347 \\
\text { RR } 1.09,95 \% \mathrm{Cl} \\
0.92 \text { to } 1.30\end{array}$ & - \\
\hline & $\begin{array}{l}\text { Placebo cream and urea } 40 \% \\
\text { paste, } n=345\end{array}$ & & - & $18 / 330$ & $142 / 345$ & - \\
\hline \multirow[t]{8}{*}{$\begin{array}{l}\text { Gupta } \\
2006 \mathbf{b}\end{array}$} & $\begin{array}{l}1 \% \text { terbinafine nail lacquer } \\
(\mathrm{TNL}) \\
\mathrm{n}=12\end{array}$ & Daily for 6 weeks & - & $0 / 12$ & $4 / 12$ & - \\
\hline & $5 \% \mathrm{TNL}, \mathrm{n}=12$ & & - & $1 / 12$ & $5 / 12$ & - \\
\hline & $10 \%$ TNL, $\mathrm{n}=12$ & & - & $2 / 12$ & $6 / 12$ & - \\
\hline & $1 \% \mathrm{TNL}, \mathrm{n}=20$ & \multirow[t]{5}{*}{ Daily for 12 weeks } & - & $1 / 20$ & $6 / 20$ & - \\
\hline & $5 \% \mathrm{TNL}, \mathrm{n}=20$ & & - & $1 / 20$ & $8 / 20$ & - \\
\hline & $10 \% \mathrm{TNL}, \mathrm{n}=20$ & & - & $0 / 20$ & $11 / 20$ & - \\
\hline & Ciclopirox $8 \%$ lacquer, $\mathrm{n}=12$ & & - & $0 / 12$ & $5 / 12$ & - \\
\hline & Placebo lacquer, $\mathrm{n}=12$ & & - & $0 / 12$ & $4 / 12$ & - \\
\hline $\begin{array}{l}\text { Kumar } \\
2001^{\mathbf{b}}\end{array}$ & $\begin{array}{l}\text { Herbal capsule and cream, } \\
n=21\end{array}$ & $\begin{array}{l}\text { Twice daily for } 12 \\
\text { weeks }\end{array}$ & - & - & $\begin{array}{l}17 / 21 \\
\text { RR } 5.94,95 \% \mathrm{Cl} \\
2.03 \text { to } 17.34\end{array}$ & - \\
\hline
\end{tabular}


Table 3. Summary of single studies (Continued)

Placebo capsule and cream,

$\mathrm{n}=22$

\begin{tabular}{|c|c|c|c|c|c|c|}
\hline \multirow[t]{2}{*}{$\begin{array}{l}\text { Lahfa } \\
2013^{\mathbf{b}}\end{array}$} & $\begin{array}{l}40 \% \text { urea with plastic dressing } \\
+ \text { bifonazole, } n=53\end{array}$ & $\begin{array}{l}\text { Urea combination } \\
\text { daily for } 3 \text { weeks, } \\
\text { then bifonazole } \\
\text { cream for } 8 \text { weeks }\end{array}$ & $\begin{array}{l}13 / 53 \\
\text { RR } 1.28, \\
95 \% \mathrm{Cl} \\
0.61 \text { to } \\
2.65\end{array}$ & $\begin{array}{l}4 / 53 \\
\text { RR } 0.56,95 \% \\
\text { CI } 0.17, \text { to } .80\end{array}$ & $\begin{array}{l}20 / 53 \\
\text { RR } 0.70,95 \% \mathrm{Cl} \\
0.46 \text { to } 1.07\end{array}$ & $\begin{array}{l}19 / 53 \\
\text { RR } 1.33, \\
95 \% \mathrm{Cl} \\
0.75 \text { to } \\
2.37\end{array}$ \\
\hline & $\begin{array}{l}40 \% \text { urea with } 1 \% \text { bifonazole }+ \\
\text { bifonazole, } n=52\end{array}$ & & $10 / 52$ & $7 / 52$ & $28 / 52$ & $14 / 52$ \\
\hline
\end{tabular}

$\begin{array}{lll}\begin{array}{l}\text { Montana } \\ 1994\end{array} & \text { Fungoid tincture, } \mathrm{n}=10 & \begin{array}{l}\text { Twice daily for } 12 \\ \text { months }\end{array}\end{array}$

$9 / 20$

Culture: $5 / 10$

$\mathrm{KOH}: 5 / 10$

RR $5.00,95 \% \mathrm{Cl}$

0.70 to 35.50

Vehicle, $n=10$

$-$

Culture: $1 / 10$

$\mathrm{KOH}: 1 / 10$

\begin{tabular}{|c|c|c|c|c|c|c|}
\hline \multirow[t]{2}{*}{$\begin{array}{l}\text { NC- } \\
\text { T02343627a }\end{array}$} & NVXT solution, $\mathrm{n}=35$ & $\begin{array}{l}\text { Once daily for } 60 \\
\text { days }\end{array}$ & - & $\begin{array}{l}7 / 35 \\
\text { RR } 0.75,95 \% \\
\text { Cl } 0.16 \text { to } \\
3.52\end{array}$ & $\begin{array}{l}14 / 35 \\
\text { RR } 3.33,95 \% \mathrm{Cl} \\
0.63 \text { to } 17.57\end{array}$ & - \\
\hline & Vehicle, $n=12$ & & - & $3 / 12$ & $2 / 12$ & - \\
\hline \multirow[t]{3}{*}{$\begin{array}{l}\text { NC- } \\
\text { T02933879a }\end{array}$} & NVXT topical solution, $n=64$ & $\begin{array}{l}\text { Once daily for } 8 \\
\text { weeks }\end{array}$ & $1 / 64$ & $19 / 64$ & $7 / 64$ & $1 / 64$ \\
\hline & NVXT topical solution, $\mathrm{n}=61$ & $\begin{array}{l}\text { Once daily for } 2 \times 8 \text { - } \\
\text { week periods, sepa- } \\
\text { rated by } 32 \text { weeks }\end{array}$ & $\begin{array}{l}0 / 61 \\
\text { RR } 0.31 \text {, } \\
95 \% \mathrm{Cl} 0.0 \\
\text { to } 7.50 \text { vs } \\
\text { placebo }\end{array}$ & $\begin{array}{l}14 / 62 \\
\text { RR } 1.01 \\
95 \% \mathrm{Cl} 0.52 \\
\text { to } 1.96 \text { vs } \\
\text { placebo }\end{array}$ & $\begin{array}{l}13 / 61 \\
\text { RR } 1.01,95 \% \mathrm{Cl} \\
0.50 \text { to } 2.03 \mathrm{vs} \\
\text { placebo }\end{array}$ & $\begin{array}{l}1 / 61 \\
\text { RR } 0.93, \\
95 \% \mathrm{Cl} \\
0.06 \text { to } \\
14.59 \text { vs } \\
\text { placebo }\end{array}$ \\
\hline & Placebo, $n=57$ & & $1 / 57$ & $13 / 58$ & $12 / 57$ & $1 / 57$ \\
\hline \multirow[t]{2}{*}{$\begin{array}{l}\text { Syed } \\
1998 \boldsymbol{a}\end{array}$} & $\begin{array}{l}\text { Butenafine } \mathrm{HCl} 2 \% \text { and } 20 \% \\
\text { urea, } \\
n=50\end{array}$ & Twice daily for 7 days & $\begin{array}{l}44 / 50 \\
\mathrm{RR} 19.20, \\
95 \% \mathrm{Cl} \\
1.28 \text { to } \\
288.53\end{array}$ & $\begin{array}{l}5 / 50 \\
\text { RR } 2.37,95 \% \\
\text { CI } 0.14 \text { to } \\
39.84\end{array}$ & - & - \\
\hline & Placebo, $n=10$ & & $0 / 10$ & $0 / 10$ & - & - \\
\hline $\begin{array}{l}\text { Syed } \\
1999 a\end{array}$ & $\begin{array}{l}\text { Butenafine } \mathrm{HCl} 2 \% \text { and } 5 \% \\
\text { Melaleuca alternifolia oil, } \\
\mathrm{n}=40\end{array}$ & $\begin{array}{l}3 \text { times/day, max. } 8 \\
\text { weeks with occlusive } \\
\text { dressing }\end{array}$ & $\begin{array}{l}32 / 40 \\
\mathrm{RR} 33.29 \\
95 \% \mathrm{Cl}\end{array}$ & $\begin{array}{l}4 / 40 \\
\text { RR } 4.61,95 \% \\
\text { CI } 0.26 \text { to } \\
81.63\end{array}$ & - & - \\
\hline
\end{tabular}


Table 3. Summary of single studies (Continued)

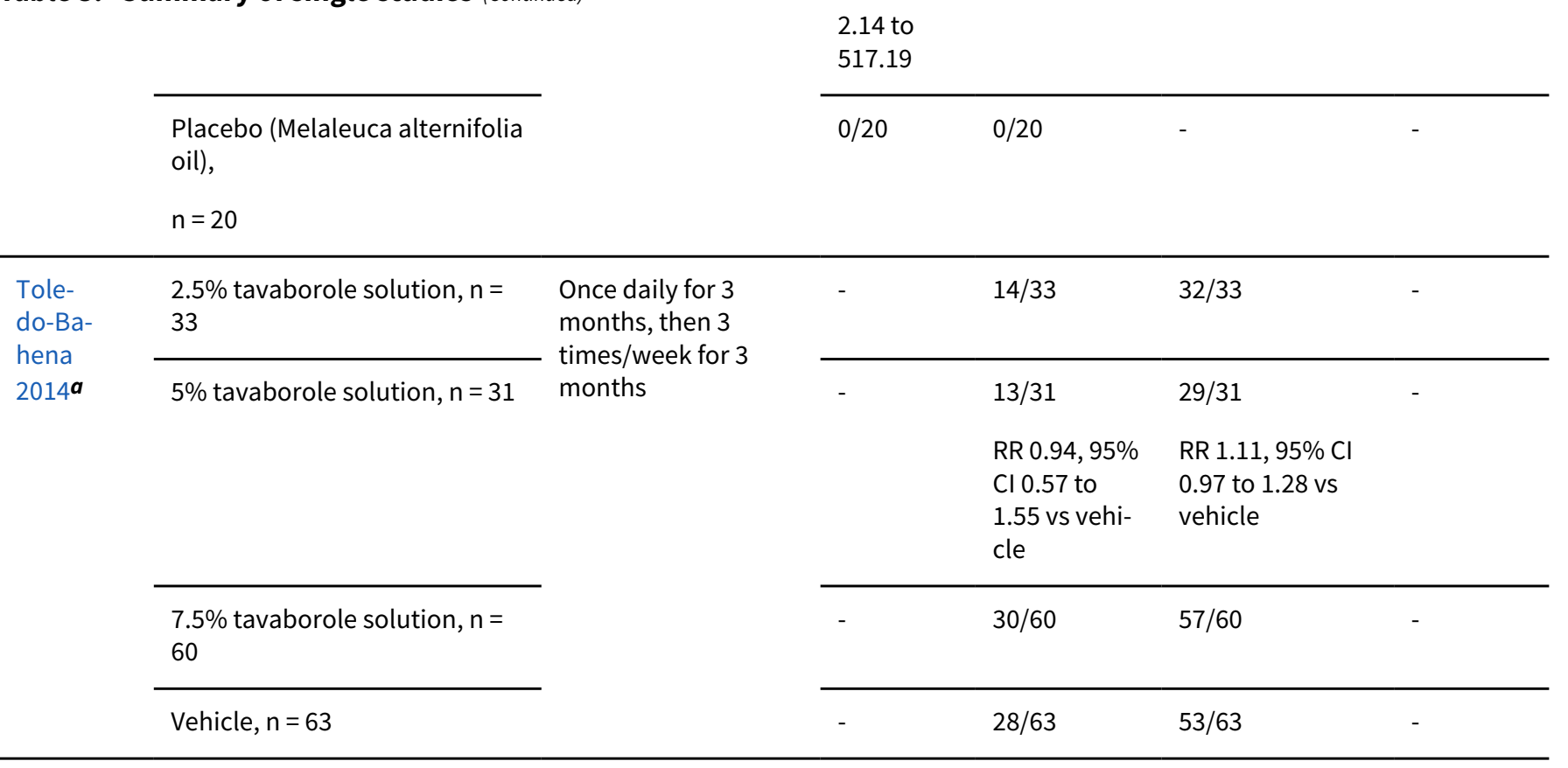

\section{Topical treatment versus topical treatment}

\begin{tabular}{lll}
\hline Buck & $100 \%$ Melaleuca alternifolia & Twice daily for \\
$1994 a$ & (Tea tree oil), $\mathrm{n}=64$ & months
\end{tabular}

Topical treatment versus topical treatment

1994a (Tea tree oil), $\mathrm{n}=64 \quad$ months
$1 \%$ clotrimazole solution, $n=$ 53

\begin{tabular}{|c|c|c|c|c|c|c|}
\hline \multirow[t]{2}{*}{$\begin{array}{l}\text { Elewski } \\
2013 \mathrm{~b} b\end{array}$} & TNS, $n=507$ & $\begin{array}{l}\text { Once daily for } 48 \\
\text { weeks }\end{array}$ & $\begin{array}{l}6 / 507 \\
\text { RR 1.24, } \\
95 \% \mathrm{Cl} \\
0.38 \text { to } \\
4.02\end{array}$ & $\begin{array}{l}285 / 493 \\
\text { RR } 1.02,95 \% \\
\text { Cl } 0.91 \text { to } \\
1.13\end{array}$ & $\begin{array}{l}82 / 507 \\
\text { RR } 1.03,95 \% \mathrm{Cl} \\
0.78 \text { to } 1.36\end{array}$ & $\begin{array}{l}8 / 507 \\
\text { RR 0.82, } \\
95 \% \mathrm{Cl} \\
0.33 \text { to } \\
2.07\end{array}$ \\
\hline & $\begin{array}{l}\text { Amorolfine } 5 \% \text { nail lacquer, } n \\
=522\end{array}$ & $\begin{array}{l}\text { Twice weekly with } \\
\text { nail filing for } 48 \\
\text { weeks }\end{array}$ & $5 / 522$ & $291 / 512$ & $82 / 522$ & $10 / 522$ \\
\hline \multirow[t]{3}{*}{$\begin{array}{l}\text { Hart- } \\
\text { mane } \\
2013\end{array}$} & $\mathrm{P}-30585 \%, \mathrm{n}=18$ & Daily for 24 weeks & - & - & $\begin{array}{l}\text { Culture: } 18 / 18 \\
\mathrm{KOH}: 14 / 18\end{array}$ & - \\
\hline & P-3058 10\%, n = 19 & & - & - & $\begin{array}{l}\text { Culture: } 16 / 19 \\
\mathrm{KOH}: 13 / 19\end{array}$ & - \\
\hline & P-3058 10\%, $n=20$ & Weekly for 24 weeks & - & - & $\begin{array}{l}\text { Culture: } 18 / 20 \\
\mathrm{KOH}: 15 / 20\end{array}$ & - \\
\hline $\begin{array}{l}\text { Paul } \\
2013^{\mathbf{b}}\end{array}$ & $\begin{array}{l}\text { Chemical nail avulsion } \\
\text { (RV4104A ointment) + ci- }\end{array}$ & $\begin{array}{l}\text { Avulsion ointment } \\
\text { for } 3 \text { weeks, then } \\
\text { cream for } 8 \text { weeks, }\end{array}$ & - & $13 / 72$ & $\begin{array}{l}35 / 71 \\
\text { RR } 1.46,95 \% \mathrm{Cl} \\
0.98 \text { to } 2.18\end{array}$ & - \\
\hline
\end{tabular}

Topical and device-based treatments for fungal infections of the toenails (Review) 
Table 3. Summary of single studies (Continued)

$\begin{array}{lll}\text { clopirox cream + ciclopirox lac- } & \text { then lacquer for } 25 & \mathrm{RR} 0.37,95 \% \\ \text { quer, } \mathrm{n}=71 & \text { weeks } & \mathrm{Cl} 0.21 \text { to } \\ & & 0.64\end{array}$

\begin{tabular}{|c|c|c|c|}
\hline Amorolfine nail lacquer, $\mathrm{n}=71$ & $\begin{array}{l}\text { Twice weekly for } 36 \\
\text { weeks }\end{array}$ & $34 / 70$ & $24 / 71$ \\
\hline
\end{tabular}

\begin{tabular}{|c|c|c|c|c|c|c|}
\hline \multirow[t]{2}{*}{$\begin{array}{l}\text { Romero- } \\
\text { Cerecero } \\
2008 \text { c }\end{array}$} & $\begin{array}{l}\text { A. pichinchensis extract } 10 \%, \mathrm{n} \\
=55\end{array}$ & $\begin{array}{l}3 \text { times/week for } 1 \text { st } \\
\text { month; } 2 \text { times/week } \\
\text { for } 2 \text { nd month; week- } \\
\text { ly for } 4 \text { months }\end{array}$ & $\begin{array}{l}27 / 55 \\
\text { RR } 0.90 \\
95 \% \mathrm{Cl} \\
0.63 \text { to } \\
1.29\end{array}$ & $\begin{array}{l}0 / 55 \\
\text { RR } 0.14,95 \% \\
\text { CI } 0.01 \text { to } \\
2.70\end{array}$ & $\begin{array}{l}29 / 55 \\
\text { RR } 0.97,95 \% \mathrm{Cl} \\
0.68 \text { to } 1.37\end{array}$ & $\begin{array}{l}35 / 55 \\
\text { RR } 0.92, \\
95 \% \mathrm{Cl} \\
0.71 \text { to } \\
1.20\end{array}$ \\
\hline & Ciclopirox 8\%, $\mathrm{n}=55$ & & $30 / 55$ & $3 / 55$ & $30 / 55$ & $38 / 55$ \\
\hline \multirow[t]{2}{*}{$\begin{array}{l}\text { Romero- } \\
\text { Cerecero } \\
2009\end{array}$} & $\begin{array}{l}\text { A. pichinchensis extract } 12.6 \% \text {, } \\
\mathrm{n}=62\end{array}$ & 6 months & $\begin{array}{l}37 / 62 \\
\text { RR } 0.94, \\
95 \% \mathrm{Cl} \\
0.71 \text { to } \\
1.25\end{array}$ & $0 / 62$ & - & $\begin{array}{l}37 / 62 \\
\text { RR 0.94, } \\
95 \% \mathrm{Cl} \\
0.71 \text { to } \\
1.25\end{array}$ \\
\hline & $\begin{array}{l}\text { A. pichinchensis extract } 16.8 \% \text {, } \\
\mathrm{n}=60\end{array}$ & & $38 / 60$ & $0 / 60$ & - & $38 / 60$ \\
\hline \multirow[t]{2}{*}{$\begin{array}{l}\text { Schalka } \\
2012 \boldsymbol{a}\end{array}$} & Ciclopirox nail lacquer, $\mathrm{n}=20$ & Daily for 6 months & - & $0 / 20$ & $\begin{array}{l}14 / 20 \\
\mathrm{RR} 0.98,95 \% \mathrm{Cl} \\
0.66,1.45\end{array}$ & - \\
\hline & Ciclopirox nail lacquer, $\mathrm{n}=21$ & $\begin{array}{l}3 \text { times/week for } 1 \text { st } \\
\text { month; } 2 \text { times/week } \\
\text { for } 2 \text { nd month; week- } \\
\text { ly for } 4 \text { months }\end{array}$ & - & $0 / 21$ & $15 / 21$ & - \\
\hline \multirow[t]{4}{*}{$\begin{array}{l}\text { Tschen } \\
2013^{\mathbf{b}}\end{array}$} & $\begin{array}{l}\text { Efinaconazole } 10 \% \text { solution } \\
\text { without semi-occlusion, } n=39\end{array}$ & Daily for 36 weeks & $10 / 39$ & $30 / 39$ & $34 / 39$ & - \\
\hline & $\begin{array}{l}\text { Efinaconazole } 10 \% \text { solution } \\
\text { with semi-occlusion, } n=36\end{array}$ & & $8 / 36$ & $25 / 36$ & $30 / 36$ & - \\
\hline & $\begin{array}{l}\text { Efinaconazole } 5 \% \text { solution } \\
\text { without semi-occlusion, } n=38\end{array}$ & & $6 / 38$ & $25 / 38$ & $33 / 38$ & - \\
\hline & Vehicle, $n=22$ & & $2 / 22$ & $14 / 22$ & $16 / 22$ & - \\
\hline
\end{tabular}

\section{Devices}

\begin{tabular}{|c|c|c|c|c|c|c|}
\hline \multirow[t]{2}{*}{$\begin{array}{l}\text { Amichai } \\
2010 \text { c }\end{array}$} & $\begin{array}{l}1 \% \text { terbinafine } \mathrm{HCl} \text { with ion- } \\
\text { tophoretic patch, } \mathrm{n}=20\end{array}$ & $\begin{array}{l}\text { Overnight } 5 \text { days/ } \\
\text { week, } 4 \text { weeks }\end{array}$ & - & $2 / 38$ & $\begin{array}{l}16 / 20 \\
\text { RR } 1.80,95 \% \mathrm{Cl} \\
1.03 \text { to } 3.15\end{array}$ & - \\
\hline & $\begin{array}{l}1 \% \text { terbinafine } \mathrm{HCl} \text { patch with- } \\
\text { out iontophoresis, } \mathrm{n}=18\end{array}$ & & - & & $8 / 18$ & - \\
\hline $\begin{array}{l}\text { Waibel } \\
2013^{a}\end{array}$ & $1064 \mathrm{~nm}$ laser, $\mathrm{n}=7$ & Weekly for 4 weeks & - & $0 / 7$ & $7 / 7$ & - \\
\hline
\end{tabular}


Table 3. Summary of single studies (Continued)

$1319 \mathrm{~nm}$ laser, $\mathrm{n}=7$

BroadBand light, $\mathrm{n}=7$

\begin{tabular}{llll}
- & $0 / 7$ & $6 / 7$ & - \\
\hline- & $0 / 7$ & $7 / 7$ & -
\end{tabular}

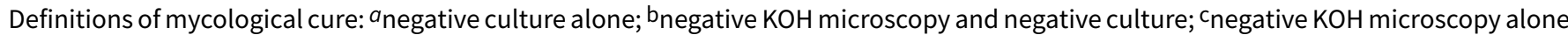

\section{APPEN DICES}

\section{Appendix 1. Cochrane Skin Specialised Register (CRSW) search strategy}

onychomycos ${ }^{\star}$ or tinea unguium or ((fungal or fungus) near (toenail ${ }^{\star}$ or toe ${ }^{\star}$ or nail $\left.{ }^{\star}\right)$ ) or (ringworm near (toenail ${ }^{\star}$ or toe* or nail $\left.^{\star}\right)$ )

\section{Appendix 2. CENTRAL (Cochrane Library) search strategy}

\#1 MeSH descriptor: [Onychomycosis] explode all trees

\#2 ((fungal or fungus) near (toenail* or toe* or nail*)):ti,ab,kw

\#3 (ringworm near (toenail ${ }^{\star}$ or toe or nail $\left.\left.^{\star}\right)\right):$ ti,ab,kw

\#4 onychomycos*:ti,ab,kw

$\# 5$ tinea unguium:ti,ab,kw

\#6 $\{$ or \#1-\#5\}

\section{Appendix 3. MEDLINE (Ovid) search strategy}

1. exp Onychomycosis/

2. ((fungal or fungus) adj4 (toenail\$ or toe\$ or nail\$)).mp.

3. (ringworm adj4 (toenail\$ or toe $\$$ or nail\$)).mp.

4. Onychomycos\$.mp.

5. tinea unguium.mp.

6. or/1-5

7. randomised controlled trial.pt.

8. controlled clinical trial.pt.

9. randomized.ab.

10. placebo.ab.

11. clinical trials as topic.sh.

12. randomly.ab.

13. trial.ti.

14. 7 or 8 or 9 or 10 or 11 or 12 or 13

15. exp animals/ not humans.sh.

16. 14 not 15

17.6 and 16

[Lines 7-16: Cochrane Highly Sensitive Search Strategy for identifying randomised trials in MEDLINE: sensitivity- and precision-maximizing version (2008 revision)]

\section{Appendix 4. Embase (Ovid) search strategy}

1. exp onychomycosis/ or exp toenail onychomycosis/

2. ((fungal or fungus) adj4 (toenail\$ or toe\$ or nail\$)).mp.

3. (ringworm adj4 (toenail\$ or toe $\$$ or nail\$)).mp.

4. onychomycos\$.mp.

5. tinea unguium.mp.

6. or/1-5

7. crossover procedure.sh.

8. double-blind procedure.sh.

9. single-blind procedure.sh.

10. (crossover\$ or cross over\$).tw.

11. placebo\$.tw.

12. (doubl\$ adj blind\$).tw.

13. allocat\$.tw.

14. trial.ti.

Topical and device-based treatments for fungal infections of the toenails (Review) 
15. randomised controlled trial.sh.

16. random\$.tw.

17. or/7-16

18. exp animal/ or exp invertebrate/ or animal experiment/ or animal model/ or animal tissue/ or animal cell/ or nonhuman/

19. human/ or normal human/

20. 18 and 19

21. 18 not 20

22. 17 not 21

23. 6 and 22

\section{Appendix 5. LILACS search strategy}

onychomycos\$ or onicomicosis

We searched using these terms and the Controlled clinical trials topic-specific query filter.

\section{CONTRIBUTIONS OF AUTHORS}

KF was the contact person with the editorial base.

$A K G, K F$ co-ordinated contributions from the co-authors and wrote the final draft of the review.

KF, SV screened papers against eligibility criteria.

$\mathrm{KF}, \mathrm{RM}, \mathrm{SV}$ obtained data on ongoing and unpublished studies.

$\mathrm{KF}, \mathrm{RM}$ appraised the quality of papers.

KF, RM, SV extracted data for the review and sought additional information about papers.

KF, RM, SV entered data into Review Manager 5.

AKG, KF analysed and interpreted data.

$\mathrm{EV}, \mathrm{KF}$ worked on the Methods sections.

AKG, KF drafted the clinical sections of the Background and responded to the clinical comments of the referees.

$\mathrm{EV}, \mathrm{KF}$ responded to the methodology and statistics comments of the referees.

DJ was the consumer co-author and checked the review for readability and clarity, as well as ensuring outcomes are relevant to consumers. AKG is the guarantor of the update.

\section{Disclaimer}

This project was supported by the National Institute for Health Research, via Cochrane Infrastructure funding to the Cochrane Skin Group. The views and opinions expressed therein are those of the authors and do not necessarily reflect those of the Systematic Reviews Programme, NIHR, NHS or the Department of Health.

\section{DECLARATIONS OF INTEREST}

Kelly Foley, Aditya Gupta, Rachel Mays, and Sarah Versteeg are employed by Mediprobe Research Inc. Mediprobe Research Inc. is not a commercial organisation, nor is it a contract research organisation.

In addition to Dr Gupta's dermatology practice, he conducts clinical trials and these are done through Dr AK Gupta Medicine Professional Corporation, previously known as Mediprobe Research. In Canada, all physicians work through Professional Corporations, and Dr Gupta does the same. Mediprobe Research is the name we continue to use because we have done so for years, but the official corporate entity is Dr AK Gupta Medicine Professional Corporation. We have not conducted clinical trials for tinea pedis in the past three years, and Dr Gupta is currently recruiting for two trials using topical treatments for onychomycosis for Valeant Canada and Moberg Pharma.

Dr Gupta has received honoraria through his institution for educational presentations to physicians from Valeant Canada.

Elmer Villanueva: nothing to declare.

Denny John: nothing to declare.

Aditya Gupta, lead author on the protocol for this Cochrane Review, became conflicted in terms of Cochrane's commercial sponsorship policy and has been replaced by Kelly Foley, as lead author. Kelly Foley has no relevant financial conflict of interest, and in the judgement of Cochrane Skin's editorial team, she has made a contribution equal to Aditya Gupta, which justifies her position as first author. This change has been discussed with and approved by the Funding Arbiters.

\section{SOURCES OF SUPPORT}

\section{Internal sources}

- No sources of support supplied 


\section{External sources}

- The National Institute for Health Research (NIHR), UK.

The NIHR, UK, is the largest single funder of the Cochrane Skin Group.

\section{DIFFERENCES BETWEEN PROTOCOLAND REVIEW}

Types of interventions. The protocol did not include 'vehicle' as a comparator in the inclusion criteria. This was added to the intervention review. This was a simple oversight on the part of the authors, as vehicle should have been included in the protocol. Commonly, placebo and vehicle are used interchangeably but they are different. For reference, placebo has no pharmacological activity (e.g. sugar pill) whereas a vehicle is meant to be similar to the drug formulation in ingredients, with the exception that the active drug is not included in the vehicle.

Data collection and analysis. In the protocol, authors FS, DD, DL, and MP were designated as the individuals that would select studies, perform data extraction and management, and evaluate risks of bias. These authors were unavailable to continue working on the intervention review and were replaced with authors KF, RM, and SV.

Assessment of risk of bias in included studies. Assessment of other potential sources of bias was not included in the protocol and is assessed in the review. We assessed other bias in the review because it was included in the RevMan 'Risk of bias' tool. We used this domain to assess whether there was selective reporting of subgroups and whether multiple intervention trials reported outcomes for all study arms, as suggested in Chapter 16 of the Cochrane Handbook (Higgins 2011).

Measures of treatment effect. In the protocol, we stated that continuous outcomes would be analysed as a mean difference (MD) with a $95 \% \mathrm{Cl}$. In the review, we did not do this because there were no continuous data to analyse. In the protocol, we stated that time to recurrence would be measured using the hazard ratio and standard errors collected from Cox proportional hazards models. In the review, we did not do this because time to recurrence data were not reported in included studies.

Unit of analysis issues. The protocol did not address studies with multiple intervention arms. We added to the review "Where studies had multiple intervention arms, all arms are mentioned in the Characteristics of included studies table." The protocol states, "The participant was the unit of analysis, with clinical trials designating one target toenail per participant as the unit of analysis. We did not consider nail data in clinical studies that used individual nails as the unit of analysis, including multiple toenails per participant in their data set." The review still states this, with the addition "The exception to this was in the case of studies where multiple toenails per participant were included if outcomes were reported per participant." This was done so that device studies, specifically laser, could be eligible for inclusion in the review.

Assessment of heterogeneity. We originally planned not to report meta-analyses for comparisons where 12 is greater than $75 \%$. We decided not to do this because $\mathrm{I}^{2}$ thresholds are guidelines and it was important to show all of the data. Instead, the large heterogeneity was explicitly addressed in the Results section for each comparison where it was present and explanations provided as to why the heterogeneity could have been large. Where possible, we performed a sensitivity analysis to determine if removing studies would affect the heterogeneity and this is also reported in the Results section. Lastly, we downgraded the quality of evidence for inconsistency when heterogeneity was large.

Data synthesis. There were no continuous data to analyse in this review. Thus we did not calculate mean differences (continuous data) using the inverse-variance model. The protocol did not identify how studies with multiple interventions arms would be handled. We therefore added to the review "For studies with multiple intervention arms, the relevant interventions were identified. Where possible, intervention arms were combined so that a single comparison was included in the meta-analysis. When this was not possible, the relevant intervention arms were used in the analysis and identified in the footnotes of the forest plot". We originally planned to conduct metaanalyses for comparisons where there were at least three studies ("Where meta-analysis is possible (e.g. at least three trials), we will conduct the analyses using a random-effects model"). We instead conducted meta-analyses for comparisons where there were at least two studies. This was done because there was a lack of studies for each treatment comparison. In the protocol, we state "If meta-analysis is not possible due to the number of trials (less than three) or considerable heterogeneity (greater than $75 \%$ ), we will assess the studies for the data collected qualitatively." In the review, we reported data qualitatively if the number of trials was fewer than two (see earlier in this section) and we reported meta-analyses where heterogeneity was greater than $75 \%$ and discussed qualitatively (see 'Assessment of heterogeneity' in this section). There were no data for the 'Time to recurrence' outcome. Thus we did not summarise time to recurrence by calculating random-effects estimates of the pooled hazard ratio using the generic inverse-variance method.

Sensitivity analysis. The protocol states that we would perform sensitivity analyses to determine the impact of removing studies with missing data, to examine if they contributed to the risk of bias. We did not do this because studies in our analyses did not have missing data.

'Summary of findings' table. The protocol states that if further subgroup analyses merit their own 'Summary of findings' table, we will include further tables. There were no subgroup analyses that warranted a separate 'Summary of findings' table in the review. The protocol states that primary outcomes will be included in the 'Summary of findings' table. We also included the secondary outcomes, mycological cure and clinical cure, in the tables because this provides readers with a more complete understanding of the findings. 